University of New Hampshire

University of New Hampshire Scholars' Repository

Spring 2016

\title{
Synthesis and Characterization of Silver Nanowire Suspensions for Printable Conductive Media
}

Shohreh Hemmati

University of New Hampshire, Durham

Follow this and additional works at: https://scholars.unh.edu/dissertation

\section{Recommended Citation}

Hemmati, Shohreh, "Synthesis and Characterization of Silver Nanowire Suspensions for Printable Conductive Media" (2016). Doctoral Dissertations. 2250.

https://scholars.unh.edu/dissertation/2250

This Dissertation is brought to you for free and open access by the Student Scholarship at University of New Hampshire Scholars' Repository. It has been accepted for inclusion in Doctoral Dissertations by an authorized administrator of University of New Hampshire Scholars' Repository. For more information, please contact Scholarly.Communication@unh.edu. 


\title{
SYNTHESIS AND CHARACTERIZATION OF SILVER NANOWIRE SUSPENSIONS FOR PRINTABLE CONDUCTIVE MEDIA
}

\author{
BY \\ SHOHREH HEMMATI \\ Baccalaureate Degree in Chemical Engineering, Arak University, Iran, 2006 \\ Master's Degree, Energy Engineering, Sharif University of Technology, Iran, 2009 \\ DISSERTATION \\ Submitted to the University of New Hampshire \\ In Partial Fulfillment of \\ the Requirements for the Degree of \\ Doctor of Philosophy \\ in \\ Chemical Engineering
}

May, 2016 


\title{
SYNTHESIS AND CHARACTERIZATION OF SILVER NANOWIRE SUSPENSIONS FOR PRINTABLE CONDUCTIVE MEDIA
}

\author{
BY \\ SHOHREH HEMMATI
}

This thesis has been examined and approved in partial fulfillment of the requirements for the degree of Doctor of Philosophy in Chemical Engineering by:

Thesis Director, Dale P. Barkey, Professor of Chemical Engineering

Nivedita Gupta, Associate Professor of Chemical Engineering

Xiaowei Teng, Associate Professor of Chemical Engineering

John McHugh, Associate Professor of Mechanical Engineering

Gonghu Li, Associate Professor of Chemistry

On April 1, 2016

Original approval signatures are on file with the University of New Hampshire Graduate School. 


\section{ACKNOWLEDGMENTS}

Completion of this doctoral dissertation was possible with the support of several people. I would like to express my sincere gratitude to all of them.

The most important acknowledgement of gratitude I wish to express is to my advisor professor Dale P. Barkey for his guidance and patience during my graduate study. It has been an honor to be his $\mathrm{PhD}$ student and be guided during $\mathrm{PhD}$ study at the Chemical Engineering Department of University of New Hampshire. He has supported me throughout my research assistantship over three years and academically through the rough road to finish this thesis as well.

I would also like to thank Dr. Nivedita Gupta, Dr. Xiaowei Teng, Dr. John McHugh, and Dr. Gonghu Li for serving on my dissertation committee. Dr. Gupta was especially helpful to give me much better understanding of the rheological science behind my research.

I am grateful to the department of Chemical Engineering, and Graduate School in UNH.

Last but not the least, I will forever be thankful to my beloved parents, Parivash and Hossein Hemmati for their love, support, and encouragement to further my education. I would not imagine my success without their support.

This work was supported by National Science Foundation (NSF) through award number CMMI120005, a GOALI grant in partnership with Conductive Compounds Inc. in Hudson, NH. 
This work also was supported by a Dissertation Year Fellowship awarded by the UNH graduate school. 


\section{TABLE OF CONTENTS}

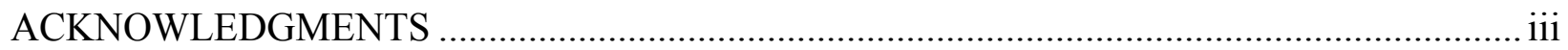

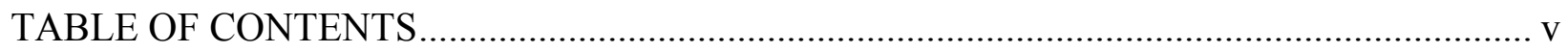

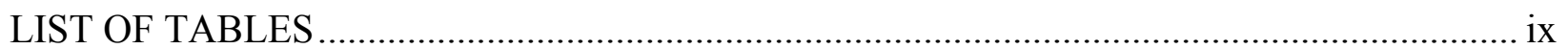

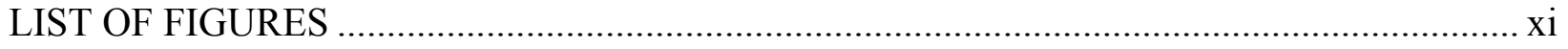

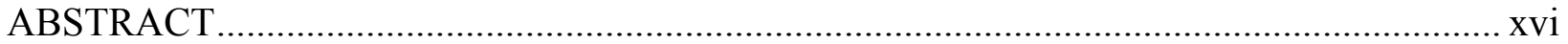

\section{CHAPTER 1: INTRODUCTION AND BACKGROUND}

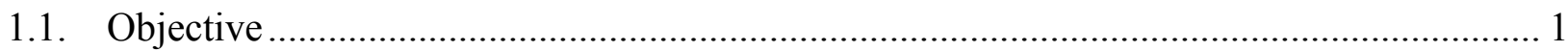

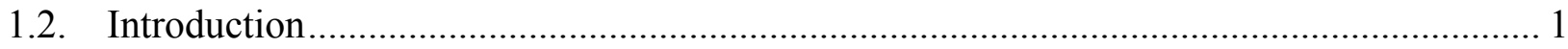

1.3. Nanotechnology and Nanoelectronics ................................................................... 2

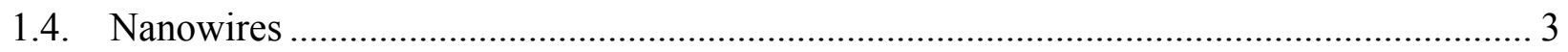

1.5. Silver Nanowires and their Applications in Electronic Devices.................................... 5

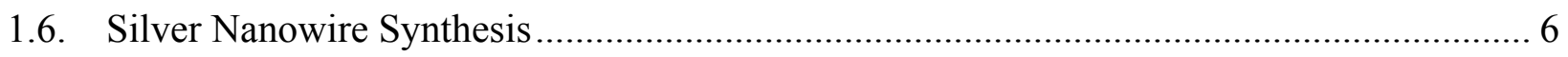

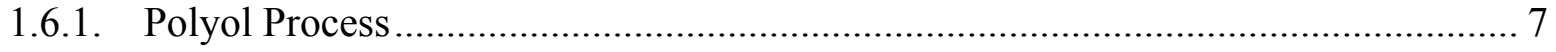

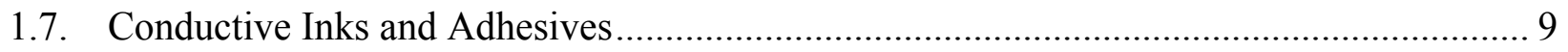

1.8. Processing of Conductive Inks and the Printing Processes ..................................... 10

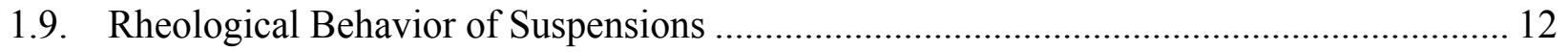

1.10. Build-up Ink Structure and Governing Equations ............................................... 20

1.11. Electrical Characteristics of Conductive Inks and Printed Patterns............................. 26

CHAPTER 2: LITERATURE REVIEW

2.1. Synthesis and Characterization of Silver Nanowires ................................................... 29 


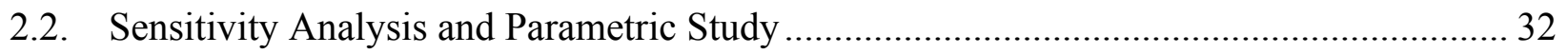

2.3. Silver Nanowire Based Conductive Inks and Adhesives for Electronic Media .................. 33

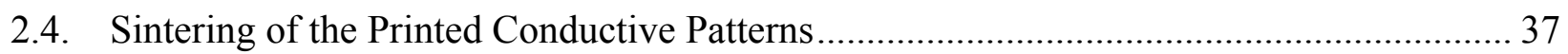

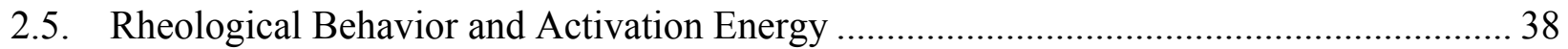

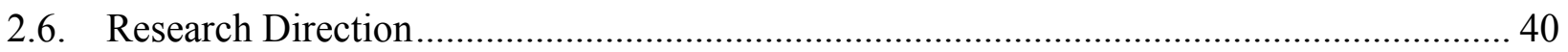

CHAPTER 3: EXPERIMENTAL APPARATUS AND PROCEDURES

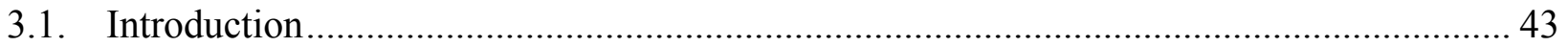

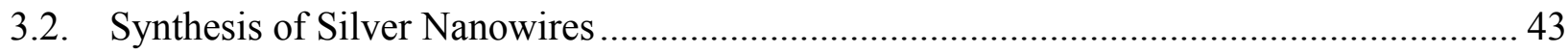

3.2.1. Batch Polyol Process............................................................................................ 43

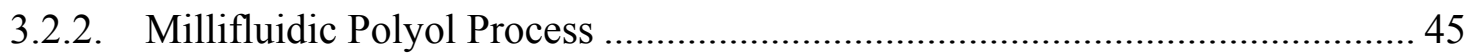

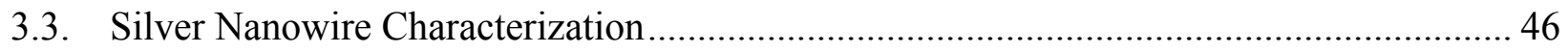

3.4. Sensitivity Analysis of the Polyol Process …………........................................................... 46

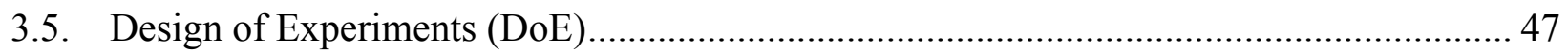

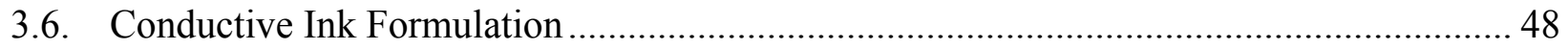

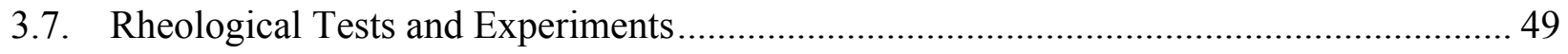

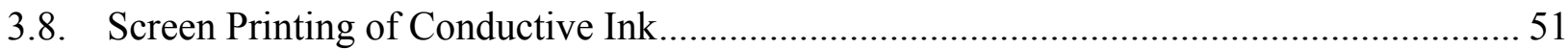

3.9. Characterization of Screen Printed Patterns ……………................................................. 51

3.10. Resistivity Measurements of the Screen Printed Patterns................................................. 51

CHAPTER 4: RESULTS AND DISCUSSIONS

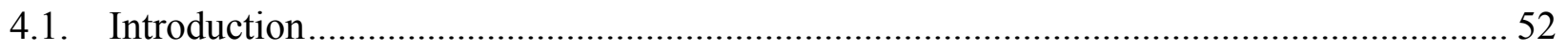

4.2. Silver Nanowire Synthesis and Characterization............................................................... 53

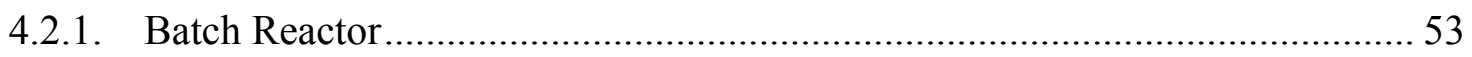

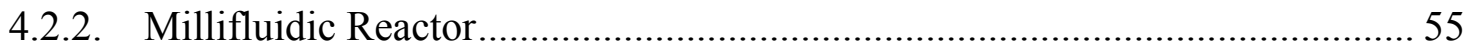




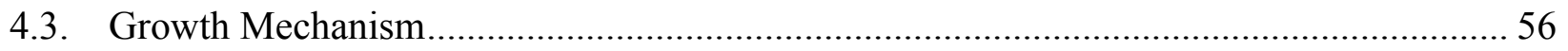

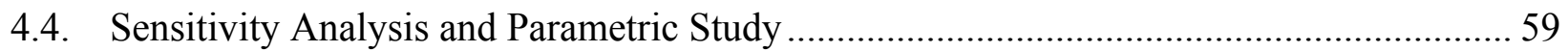

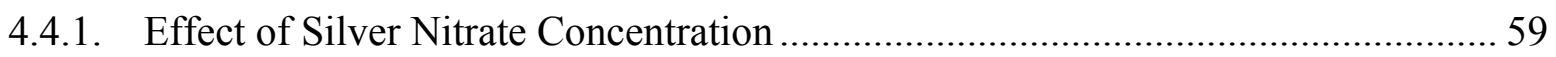

4.4.2. Effect of Salt Type, and Copper Chloride Concentration....................................... 60

4.4.3. Effect of PVP Concentration and Molecular Weight ............................................... 61

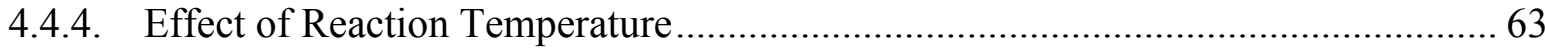

4.4.5. Effect of Sonication Time of the Silver Nitrate Solution ......................................... 64

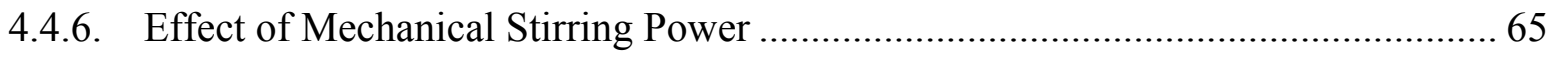

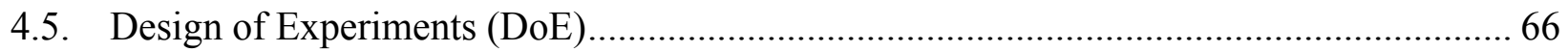

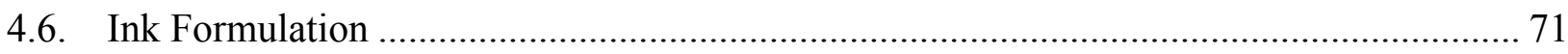

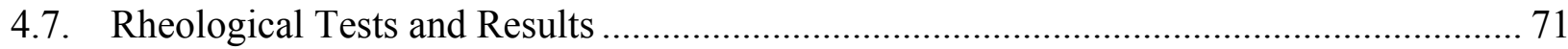

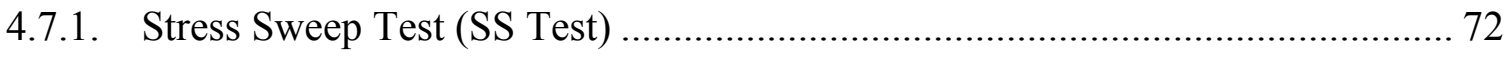

4.7.2. Steady State Flow Test (SSF Test) ............................................................... 74

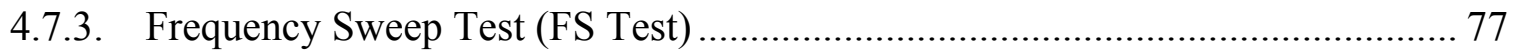

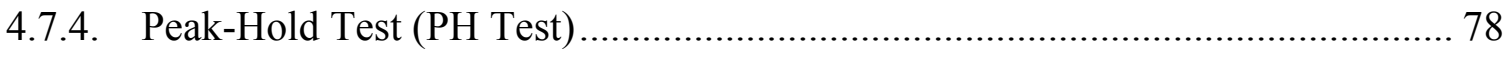

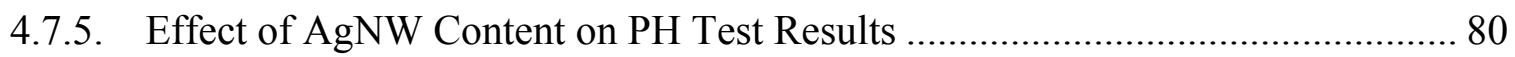

4.7.6. Comparison between Commercial and Synthesized AgNWs.............................. 81

4.7.7. Rheological Behavior of Commercial AgNW Ink during Screen Printing ......... 82

4.7.7.1. Simulation of Screen Printing (PH Test) .................................................... 83

4.7.7.2. Correlation of Build-up Structure (SE model)............................................ 96

4.7.7.3. Dilute/ Semi Dilute/ Concentrated Regimes and Transitions ..................... 110

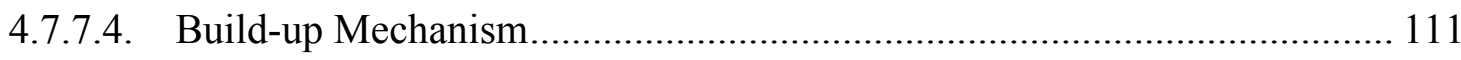

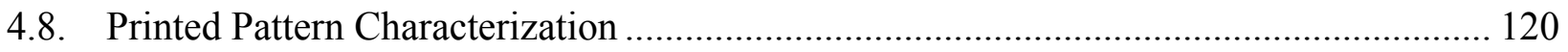




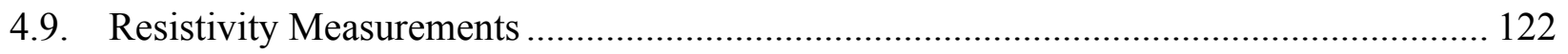
CHAPTER 5: CONCLUSONS AND RECOMMENDATIONS FOR FUTURE WORK

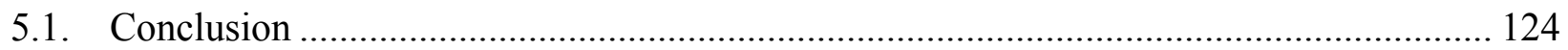

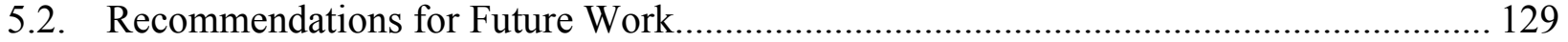




\section{LIST OF TABLES}

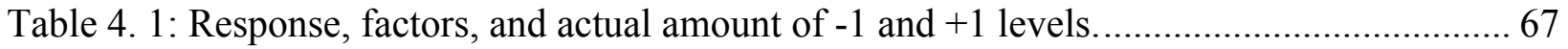

Table 4. 2: P value for yield of synthesized AgNWs surface response. ................................... 70

Table 4. 3: Average viscosity at different steps of the peak hold test for different silver nanowire (AgNWs-90) contents at several temperatures.

Table 4. 4: Average viscosity at different steps of the peak hold test for different silver nanowire (AgNWs-90) contents at two values of recovery shear rate. 94

Table 4. 5: Average viscosity at different steps of the peak hold test for different silver nanowire (AgNWs-40) contents at several temperatures. 95

Table 4. 6: Activation energy and frequency factor for different silver nanowire contents....... 109

Table 4. 7: Dilute/ semi dilute/ concentrated regime transition criteria for AgNWs-90 (d=90nm, $\mathrm{L}=30 \mu \mathrm{m})$, and AgNWs-40 (d=40nm, $\mathrm{L}=30 \mu \mathrm{m})$.

Table 4. 8: Peclet number and characteristic time of selected silver nanowire (AgNWs-90) contents at different temperatures.

Table 4. 9: Peclet number and characteristic time of selected silver nanowire (AgNWs-90) contents at different viscosity recovery shear rates.

Table 4. 10: Peclet number and characteristic time of selected silver nanowire (AgNWs-40) contents at different temperatures. 113

Table 4. 11: Activation enthalpy, and activation entropy during recovery process for different silver nanowire (AgNWs-90) contents. 120

Table 4. 12: Activation enthalpy, and activation entropy during recovery process for different silver nanowire (AgNWs-40) contents. 120

Table 4. 13: Resistivity measurement for printed patterns at several contents of AgNWs-90... 123 
Table 4. 14: Resistivity measurement for printed patterns at several contents of AgNWs-40... 123

Table 4. 15: Resistivity measurement for printed patterns synthesized AgNWs and commercial

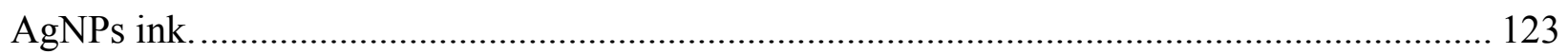




\section{LIST OF FIGURES}

Figure 1.1: Effect of Argon atmosphere in the polyol synthesis of AgNWs........................... 9

Figure 1. 2: Schematic illustration of screen printing; (a) flat-bed screen printing, (b) rotary

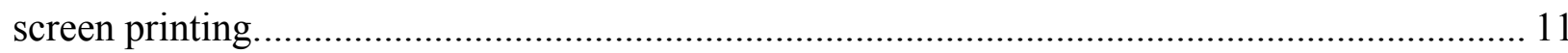

Figure 1. 3: Shear flow in typical rotational rheometer................................................... 12

Figure 1. 4: General curves of shear stress versus shear rate: (1) Newtonian, (2) shear thinning, (3) shear thickening, (4), (5) material with yield stress [33]............................................. 14

Figure 1. 5: Strain and shear rate illustration in an oscillatory flow.................................... 16

Figure 1. 6: Stress illustration in an oscillatory flow. ....................................................... 16

Figure 1. 7: Schematics of the rotational rhemeters; a: cone and plate, b: coaxial cylinder, c:

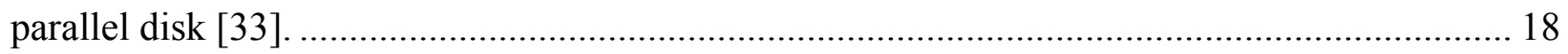

Figure 3. 1: Schematic of the small-scale batch reactor of polyol process.............................. 45

Figure 3. 2: Schematic of the millifluidic reactor of polyol process. ...................................... 46

Figure 3. 3: Schematic of the rheometer configuration. .................................................. 50

Figure 3. 4: Schematic of the screen printer. ................................................................. 51

Figure 4. 1: SEM images of the synthesized silver nanowire from a batch reactor; A: three times scale-up, B: six times scale-up, C: ten times scale-up, and D: twenty times scale-up (scale bars: $100 \mathrm{~nm})$ 54

Figure 4. 2: SEM images of synthesized AgNWs in three times batch reactor through polyol

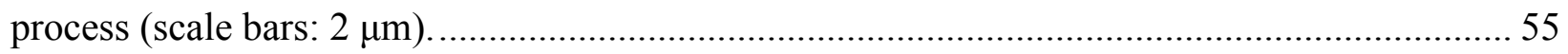

Figure 4. 3: SEM images of the synthesized silver nanowire produced in a millifluidic reactor (scale bars: $2 \mu \mathrm{m}$ ). 55 
Figure 4. 4: SEM images of the synthesized AgNWs produced in a continuous millifluidic reactor through the polyol process at several temperatures (scale bars: $5 \mu \mathrm{m}$ ). ..................... 56

Figure 4. 5: SEM images of particles recovered at various stages of the synthesis. ................. 56

Figure 4. 6: Illustration of the growth mechanism [7] ................................................... 57

Figure 4. 7: Schematic depiction of oxygen adsorption from the surface of silver seeds [7]...... 58

Figure 4. 8: Schematic of the silver nanowire growth mechanism of the polyol process. .......... 59

Figure 4. 9: $\mathrm{SEM}$ images of products at three concentrations of $\mathrm{AgNO}_{3} \mathrm{~A}: 0.047 \mathrm{M}, \mathrm{B}: 0.094 \mathrm{M}$,

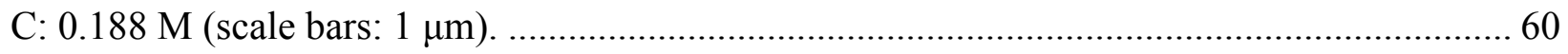

Figure 4. 10: SEM images demonstrating the effect of $\mathrm{CuCl}_{2}$ concentration; $\mathrm{A}: 0 \mathrm{mM}, \mathrm{B}: 2 \mathrm{mM}$, C: 4 mM, D: $8 \mathrm{mM}$ (scale bars: $1 \mu \mathrm{m})$

Figure 4. 11: SEM images demonstrating the effect of salt type; $\mathrm{A}: 4 \mathrm{mM} \mathrm{CuCl}$, B: $2 \mathrm{mM} \mathrm{CuCl}_{2}$ and $2 \mathrm{mM} \mathrm{NaCl}, \mathrm{C}: 4 \mathrm{mM} \mathrm{NaCl}$ (scale bars: $1 \mu \mathrm{m}$ ).

Figure 4. 12: SEM images demonstrating the effect of PVP concentration; A: $0.0735 \mathrm{M}, \mathrm{B}: 0.147$ M, C: 0.294 M, D: 0.588 M (MW: 55000) (scale bars: $1 \mu \mathrm{m})$ 62

Figure 4. 13: SEM images demonstrating the effect of PVP MW; A: 0.0735 M (MW: 55000), B: 0.0735 M (MW: 1300000), C: 0.147 M (MW: 55000), and D: 0.147 M (MW: 1300000) (scale bars: $1 \mu \mathrm{m})$. 63

Figure 4. 14: SEM images demonstrating the effect of the reaction temperature (scale bars: 1 $\mu \mathrm{m})$. 64

Figure 4. 15: SEM images demonstrating the effect of the sonication time of silver nitrate solution on the reaction products (scale bars: $1 \mu \mathrm{m}$ ). 65

Figure 4. 16: SEM images demonstrating the effect of the power of stirring; A: low, B: combination of low and high, C: high (scale bars: $100 \mathrm{~nm}$ ). 66 
Figure 4. 17: SEM images of the 27 synthesized nanostructures based on the DoE (scale bars: 2 $\mu \mathrm{m})$

Figure 4. 18: Interaction plots for the yield of $\mathrm{AgNW}$ for temperature, $\mathrm{AgNO}_{3}$ concentration, PVP concentration, and $\mathrm{CuCl}_{2}$ concentration. 70

Figure 4. 19: Maximize desirability through prediction profiler including corresponding values for parameters. 70

Figure 4. 20: SEM image of the synthesized AgNWs under the optimized conditions. 71

Figure 4. 21: $\mathrm{G}^{\prime}$ and $\mathrm{G}^{\prime}{ }^{\prime}$ versus stress for nanoparticle and nanowire suspensions obtained in the stress sweep measurements. 73

Figure 4. 22: $\mathrm{G}^{\prime}$ and $\mathrm{G}^{\prime}{ }^{\prime}$ versus stress for WB-101 ink obtained in the stress sweep measurement. 73

Figure 4. 23: Viscosity versus shear rate for nanoparticle and nanowire suspensions obtained in the steady state flow measurements. 75 Figure 4. 24: Viscosity versus shear rate for WB-101 ink obtained in the steady state flow measurement. 76 Figure 4. 25: Viscosity versus shear rate for solution of $2.5 \% \mathrm{CMC}$ and $3 \%$ dispersant obtained in the steady state flow measurement. 76 Figure 4. 26: $\mathrm{G}^{\prime}$ and $\mathrm{G}^{\prime}{ }^{\prime}$ versus angular frequency for nanoparticle and nanowire suspensions obtained in the frequency sweep measurements. 77 Figure 4. 27: $\mathrm{G}^{\prime}$ and $\mathrm{G}^{\prime} \quad$ versus angular frequency for WB-101 ink obtained in the frequency sweep measurement. 78 
Figure 4. 28: Viscosity versus time for nanoparticle and nanowire suspensions obtained in the peak hold measurements. 79

Figure 4. 29: Viscosity versus time for WB-101 ink obtained in the peak hold measurement. ... 79 Figure 4. 30: The peak hold measurement repeated at four values of silver nanowire concentration. 80

Figure 4. 31: Viscosity at different steps of printing at four values of silver nanowire concentration... 81

Figure 4. 32: The peak hold measurements for commercial and synthesized AgNWs ink. 82 Figure 4. 33: Measured viscosity versus time in the PH test for several contents of AgNWs (AgNWs-90) ink. 86

Figure 4. 34: Measured viscosity versus time in the PH test for several contents of AgNWs (AgNWs-40) ink. 91

Figure 4. 35: Measured viscosity versus time in the PH test for different AgNWs (AgNWs-90) contents at two values of shearing in viscosity recovery. 93 Figure 4. 36: Correlation of the build-up structure for different AgNWs (AgNWs-90) contents at several temperatures. 99 Figure 4. 37: Correlation of the build-up structure for different AgNWs (AgNWs-90) contents at two recovery shear rates 101 Figure 4. 38: Correlation of the build-up structure for different AgNWs (AgNWs-40) contents at several temperatures.. 106 Figure 4. 39: Temperature dependence of the characteristic time for several silver nanowire (AgNWs-90) contents. 107 
Figure 4. 40: Temperature dependence of the characteristic time for several silver nanowire (AgNWs-40) contents. 109

Figure 4. 41: Temperature dependence of the initial viscosity for different silver nanowire (AgNWs-90) contents. 115

Figure 4. 42: Temperature dependence of the recovered viscosity for different silver nanowire (AgNWs-90) contents. 116

Figure 4. 43: Temperature dependence of the initial viscosity for different silver nanowire (AgNWs-40) contents. 118

Figure 4. 44: Temperature dependence of the recovered viscosity for different silver nanowire (AgNWs-40) contents. 119

Figure 4. 45: SEM image of the printed pattern of 6 wt.\% AgNWs-90. 120

Figure 4. 46: SEM image of the printed pattern of 6 wt.\% AgNWs-40. 121

Figure 4. 47: Image of the printed patterns of synthesized AgNWs and commercial AgNPs ink. 121

Figure 4. 48: SEM image of the line part in the printed pattern of $6 \mathrm{wt} . \%$ AgNWs-90 (scale bar: $1 \mathrm{~mm})$. 121

Figure 4. 49: SEM image of the line part in the printed pattern of $6 \mathrm{wt} \%$ AgNWs-40 (scale bar: $1 \mathrm{~mm})$. 121

Figure 4. 50: SEM images of the printed patterns of AgNWs-90 with different contents of AgNWs at high magnification (scale bar: $5 \mu \mathrm{m}$ ). 122

Figure 4. 51: SEM images of the printed patterns of AgNWs-40 with different contents of AgNWs at high magnification (scale bar: $5 \mu \mathrm{m}$ ). 122 


\title{
ABSTRACT \\ SYNTHESIS AND CHARACTERIZATION OF SILVER NANOWIRE SUSPENSIONS \\ FOR PRINTABLE CONDUCTIVE MEDIA
}

\author{
by \\ Shohreh Hemmati \\ University of New Hampshire, May 2016
}

The focus of this study is on synthesis of silver nanowires (AgNWs) for conductive inks suitable for economical screen printing of conductive grids on solar panels. AgNWs were synthesized by a polyol process in both batch and continuous reactors, and a reaction mechanism was described. The morphology, diameter, and length of silver nanostructures were characterized by Scanning Electron Microscopy (SEM). The polyol synthesis was optimized by implementation of a parametric study and Design of Experiment (DoE) procedure to maximize the yield of AgNWs. AgNWs with high aspect ratio and high quality were also synthesized by the polyol process in a continuous millifluidic reactor. Water-based AgNW inks adaptable for screen printing were formulated, and their rheological behavior was investigated by several rheological tests including the Peak Hold (PH), Steady State Flow (SSF), Stress Sweep (SS), and Frequency Sweep (FS) tests. Viscosity recovery after high shear was correlated with the Stretched Exponential model (SEmo). Activation energies for flow and for viscosity recovery were measured. Together with dimensional analysis, this data was used to assess the mechanism of recovery as due to wire-wire interactions rather than Brownian motion. The inks were printed on a substrate and characterized by line definition and conductivity. 


\section{CHAPTER 1}

\section{INTRODUCTION \& BACKGROUND}

\subsection{Objective}

Growing energy demand, finite fossil fuel reserves and environmental pollution concerns point to the necessity of efficient and clean alternative energy sources. Renewable and non-carbon emitting energy sources such as solar can contribute to meeting increasing energy demand with reduced environmental cost. Harvesting solar energy as an abundant source requires a cost efficient method, and photovoltaic energy conversion is a promising technology. Solar cells and solar voltaic panels are rapidly becoming cheaper and more efficient, and there is a growing global market for units of all sizes. Fabrication of these devices in a cost effective, efficient, and environmentally friendly way is thus a priority. Screen printing of metallic conductive inks is a promising technology for fabrication of solar voltaic panel current collectors. The metallic conductive inks also can be applied for fabricating nanoscale electronic devices such as touch screens, flexible displays, thin-film transistors, organic light emitting diodes, electronic packaging, and sensors in an efficient and effective way.

\subsection{Introduction}

Metal One-Dimensional (1D) nanostructures can play a valuable role in electronic devices, and have recently gained attention due to their unique electrical, optical, thermal, and catalytic properties. Because of the high electrical and thermal conductivity of bulk silver, it is one of the most promising metal nanowire materials. Conductive inks generally contain three essential parts: metal powder or flake as conductive filler, solvent, and binder or rheological agents. Silver 
nanowires (AgNWs) hold promise as the conductive filler in conductive inks because they can be heat treated or sintered after printing at low temperature to make the patterns more conductive $[1$, 2].

Among methods for synthesis of AgNWs, the polyol process, a chemical reduction method carried out in the presence of polymeric surfactant with the assistance of an appropriate salt, is simple and inexpensive, and it provides a relatively high yield. In the polyol process a polyol solvent in the presence of polymeric capping surfactant and salt precursor is heated to synthesize colloids of metal nanostructures [1].

Various methods of printing have been used for the fabrication of printed patterns and circuits. Among these, screen printing is rapid and inexpensive [3]. In screen printing, the ink is forced through a patterned screen onto a substrate. The quality of the printed pattern depends critically on properties of the ink including rheological or flow behavior, particle size, and particle size distribution as well as on the printing process and substrate characteristics. Shear thinning behavior, meaning that the viscosity decreases with increasing shear rate, is necessary for screen printable inks. Hence, suitable inks for screen printing exhibit thixotropic rheological behavior that is time and shear dependent. The ink should have high viscosity at low shear rates when the ink is charged to the screen printer, low viscosity during printing at high shear rate, and finally rapid viscosity recovery of high viscosity after printing to ensure good line definition $[4,5]$.

\subsection{Nanotechnology and Nanoelectronics}

Nanotechnology is defined as development and use of systems, devices, and functional materials that contain matter on the nanometer size. These provide electrical, chemical, physical, and 
mechanical characteristics that may exceed those of the bulk materials. Nanoelectronics is defined as application of nanotechnology to electronic elements [6].

\subsection{Nanowires}

The transition from micro scale to nano scale particles leads to essential changes in physical and chemical characteristics due to high surface area and quantum effects [6]. 1D nanostructures including nanowires, nanorods and nanotubes have gained research interest as candidates for transparent electrode fabrication [4]. Nanowires are defined as structures which have two dimensions in the range of 1-100 nm and one dimension on a larger scale. Due to this specific configuration, they exhibit unique electrical, thermal, optical, and magnetic properties [7]. Alternatively, nanowires are wire structures with diameters in the nanometer $\left(10^{-9} \mathrm{~m}\right)$ range. Due to the specific quantum mechanic properties in this configuration, they also are known as quantum wires. There are several types of nanowires including metallic, semiconducting, and insulating [6].

Nanowires exhibit specific electrical conduction behavior due to their special quantum direction configuration. This configuration includes two quantum confined directions, as well as one unconfined direction to provide electrical conductivity. Nanowires are exceptional compared to the corresponding bulk material because of their specific electronic state density and high aspect ratio [8]. 1D nanostructures are used to create nano devices and in applications which require surface activities and quantum effects [9].

1D metallic nanowires may provide materials or devices with exceptional functionality such as high electrical conductivity, optical transparency and mechanical stiffness [2]. These metallic nanostructures perform an essential role as either active components or interconnects in nano electronic device fabrication [10]. 
Dimensional and structural characteristics of nanowires play an essential role in determining their properties and can be probed by imaging techniques. Due to the nano scale size, which is comparable to or less than the wavelength of visible light, electron microscopic techniques are required to create images of the particles. These include Scanning Electron Microscopy (SEM), Transmission Electron Microscopy (TEM), and High Resolution Transmission Electron Microscopy (HRTEM). Scanning Tunneling Probes (STP) and X-ray analysis may also be used to characterize nanowire morphology [8].

SEM is a type of electron microscopy that creates images of the sample with a focused beam of electrons. SEM images provide information about length, diameter, morphology, density, spatial distribution, and structural arrangement of nanowires [8].

TEM is a type of microscopy that provides images of the sample through transmission of an electron beam through the sample. This method provides more geometrical details at the atomic scale in comparison to SEM. HRTEM is a mode of TEM that provides direct imaging of the sample on the atomic scale. TEM and HRTEM also provide information about crystal quality and structure, grain size, and orientation of nanowire crystals [8].

Several types of scanning probe methods are used to investigate the structural, thermal, magnetic, and electric characteristics of nanowires. These techniques include Scanning Tunneling Microscopy (STM), Scanning Thermal Microscopy (SThM), Atomic Force Microscopy (AFM), and Magnetic Field Microscopy (MFM) [8]. X-Ray Diffraction (XRD), and X-Ray Energy Dispersive Analysis (EDAX) are also used to investigate the chemical composition and crystal structures of nanowires. 


\subsection{Silver Nanowires and their Applications in Electronic Devices}

Silver metal has a range of characteristics that can be adjusted, improved, or developed by control of particle morphology on the nanometer scale. Having the highest thermal and electrical conductivity among metals $\left(6.3 \times 10^{7} \mathrm{~S} / \mathrm{m}\right)$, silver is a strong candidate for electrical contacts and flexible circuits [11]. Silver has a face-centered-cubic (fcc) structure with lattice parameters similar to gold. Moreover, the $\{100\}$ facets of silver have minimal energy [12].

There is growing need to develop materials that are both optically transparent and electrically conductive with potential applications in solar cells, Light Emitting Diodes (LEDs), Organic Light Emitting Diodes (OLEDs), Liquid Crystal Displays (LCDs), flat panels or plasma displays, touch screens, optoelectronic devices, and photodiodes. AgNWs are promising candidates as conductive and transparent fillers to create films comparable to Indium-Tin Oxide (ITO), which has been widely used for this purpose [13-15].

The replacement of silver nanoparticles (AgNPs) with AgNWs may enhance the performance of devices and materials in various commercial applications $[10,16]$. AgNWs already play an essential role in fabrication of many devices such as flexible and transparent electrodes, batteries, and capacitors [2]. There are many potential applications of AgNWs, such as electronic interconnects, cell probes, sensors, transparent electrodes, photonic crystals, and plasmonic fibers [17].

As solar cells become more efficient and cheaper, and displays become bigger, there is a growing need to replace widely used materials such as ITO. Networks of random AgNWs are a possible alternative with their high electrical conductivity and optical transparency [18]. There is 
currently much research on low cost and efficient techniques for fabrication of conductive patterns, including conductive tracks, interconnects and electrodes in order to improve modern electro-optic and photovoltaic devices [19].

There are drawbacks in using ITO. For instance ITO is chemically unstable, the source of indium is limited, the cost of ITO is increasing, and ITO films are brittle. All of these reasons make it desirable to replace ITO [20]. AgNW has gained a lot of attention as a promising material to replace ITO for Transparent Conductor (TC) device fabrication in solar cells, touch screens, and displays [21, 22].

\subsection{Silver Nanowire Synthesis}

Because the properties of AgNWs depend strongly on particle size, size control is essential [9]. Moreover, the characteristics of AgNWs are strongly dependent on the distribution of individual particle dimensions. It is necessary to control the mean and variance of the diameter and length of nanowires produced in the synthesis process [13].

There are several methods for synthesis of AgNWs. These can be broadly classified as templatedirected, and template free methods. In the template-directed method, the metal is deposited chemically or electrochemically within template channels. Although AgNWs can be produced with controlled morphology by this method, the process scale is limited by cost and by availability of the template [1]. Template methods include nanolithographic, polymer or alumina, DNA or Carbon Nano Tubes (CNTs) templates. Although these methods can provide control over the AgNWs synthesis in order to control dimensions and morphology, the scale-up of these techniques is difficult. This difficulty is due to inherent properties of these methods which make them expensive and tedious to scale up. The most promising candidates for AgNWs synthesis are wet chemical methods that can provide large scale synthesis of AgNWs in a cost effective way 
[24]. Template-free synthesis methods include arc discharge of solid-liquid phase, electrochemical techniques and ultraviolet photo reduction. Among these techniques, the polyol technique is a superlative method, because it is simple, high yield, and low cost [2]. AgNWs synthesis methods can also be categorized into liquid phase and vapor phase methods. The liquid phase method has been widely used due to advantages such as the variety of suitable solvents, low cost, easy monitoring and a homogenous reaction regime [7].

Growth control to produce desired structures and morphologies is challenging. There are various methods to control the synthesis and growth of AgNWs structures including electrochemical, photochemical, wet chemical and template directed techniques, among which the polyol method has gained much more attraction due its easy operation and simplicity [17, 23].

\subsubsection{Polyol Process}

Among the processes reviewed above, the polyol process is inexpensive and simple and gives a relatively high yield [25]. Fievet et al. introduced the polyol process to synthesize metallic nanoparticles on submicron scales [16]. In the Polyol process, a polyol solvent in the presence of a polymeric capping agent and a salt precursor is heated to synthesize colloids of metal. In silver nanostructure synthesis, Ethylene Glycol (EG), Poly(Vinyl Pyrrolidone) (PVP), and silver nitrate $\left(\mathrm{AgNO}_{3}\right)$ function as the polyol, capping agent, and salt precursor, respectively [11].

The process proceeds by chemical reduction of silver ion in the presence of a polymeric capping agent. A small amount of appropriate salt can further facilitate the growth of the silver seeds to the desired shape. The EG acts as both solvent and reducing agent [25]. EG is converted to Glycol Aldehyde (GA), which is the reducing agent for silver ions according to equation 1.1 [26] 


$$
2 \mathrm{HOCH}_{2} \mathrm{CH}_{2} \mathrm{OH}+\mathrm{O}_{2} \stackrel{150^{\circ} \mathrm{C}}{\longrightarrow} 2 \mathrm{HOCH}_{2} \mathrm{CHO}+2 \mathrm{H}_{2} \mathrm{O} \text {. }
$$

Several parameters affect the yield and morphology of the synthesized AgNWs. First of all, the silver nitrate solution addition should be slow in order to avoid too-rapid formation of supersaturated Ag. Secondly, Multiply Twinned Particles (MTPs) of decahedral shape with ten $\{111\}$ facets should be produced in the reaction solution. Their formation is driven by the minimization of the surface-excess free energy. After formation of MTPs, their twinned facets provide a high local surface energy that attracts $\mathrm{Ag}$ atoms providing uniaxial growth of the MTPs. The PVP capping agent adsorbs onto and passivates the $\{100\}$ facets, which directs the $\mathrm{Ag}$ addition to the $\{111\}$ facets to facilitate uniaxial elongation $[1,17]$. Due to the presence of the capping agent, which can be chemically attached to the surface of the Ag seed by O-Ag bonding, the Ag seeds are thoroughly distributed in the reaction solution. The attachment of the PVP to the Ag seeds is shown in equation 1.2 [27].

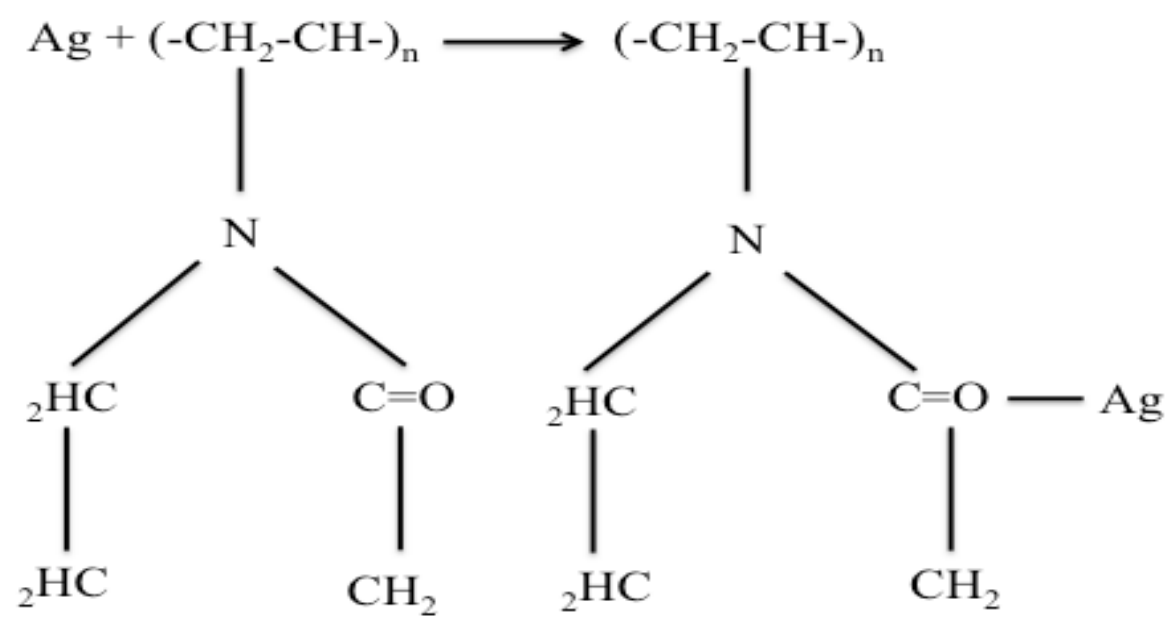


In the salt-mediated polyol process, a very small amount of appropriate salt such as $\mathrm{NaCl}$, $\mathrm{Fe}(\mathrm{NO})_{3}, \mathrm{CuCl}_{2}$, and $\mathrm{CuCl}$ plays an important role in the morphology of the synthesized AgNWs. Purging with Argon gas provides an anoxic atmosphere, and the initially formed MTPs can grow into wire shapes. Figure 1.1 shows the difference between nanostructures produced under Argon and under oxygen atmospheres [7].
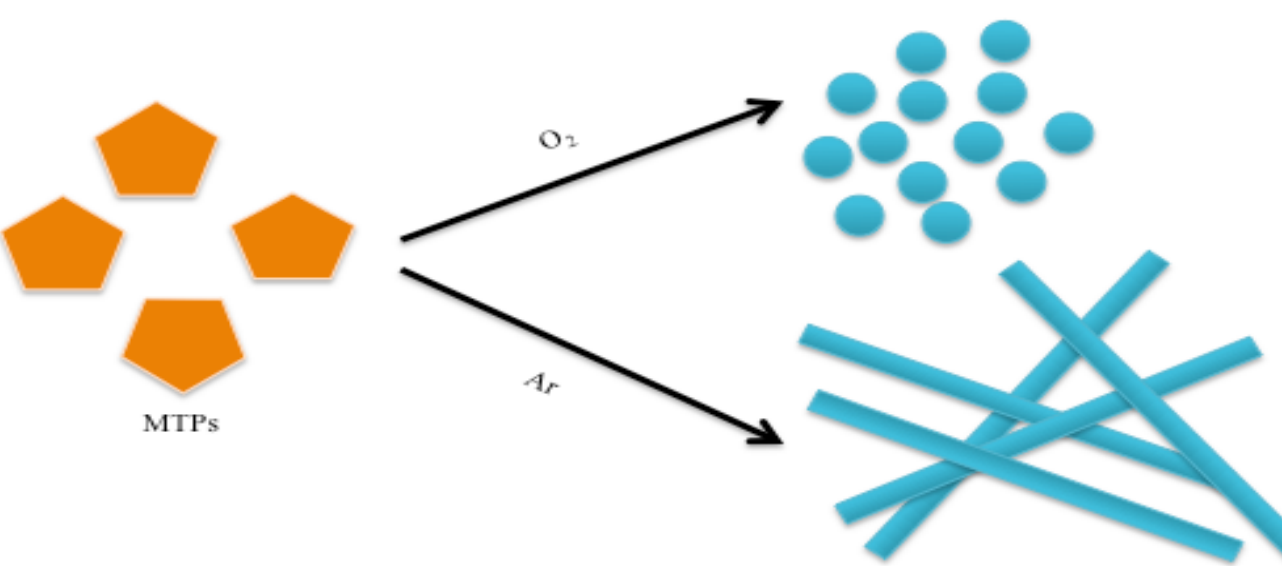

Figure 1.1: Effect of Argon atmosphere in the polyol synthesis of AgNWs.

The polyol process can be either self-seeding, or externally seeded by addition of seeds to the reaction solution. In the self-seeding technique, the growth of the seeds is based on adsorption of capping agent on a specific facet of the crystal [16]. Hence, high aspect ratio and uniform AgNWs can be synthesized in the presence or in the absence of added seeds [28].

\subsection{Conductive Inks and Adhesives}

Recent progress in printable conductive media have created routes to manufacture of flexible, light, and cost effective devices through efficient and environmentally friendly processes in comparison to the silicon-based devices [29].

Inks adaptable for printing should have specific physical and compositional properties that have strong effects on the printed patterns. The printed pattern functions and their applications dictate 
the requirements of the printing process, and consequently the ink characteristics. The rheological behavior and surface energy of the ink regulate flow, ink structure after drying, printed pattern cohesion, adhesion to the substrate and surface characteristics of the printed patterns. Nowadays, nano conductive inks that contain metallic particles of nano size are getting more attention [6]. Conductive inks adaptable for screen printing process are generally liquid inks with shear thinning behavior, which means low viscosity at high shear rate and high viscosity at low shear rate [30].

\subsection{Processing of Conductive Inks and the Printing Processes}

The fabrication of electronic devices through current silicon technology faces restrictions in flexibility and cost. Given these limitations, solution processed materials have enormous advantages [30]. Solution processed materials may contribute to creation of flexible and light weight devices and expand functionality. Solution processing may also provide excellent control over the electrical, thermal, optical and magnetic properties of the synthesized materials. The disadvantages of current silicon technology, and the advantages of solution processed materials, are evidence of the opportunities in Printed Electronics (PEs) [30].

Printing methods have been introduced since the 1950s as an alternate technique for the transfer and deposition of conductive patterns on various substrates [4].

The printing of conductive inks has attracted interest as an alternative to electroplating or lithographic processes, which are expensive, time consuming, and require multiple steps [3].

Conductive inks can be transferred and deposited on substrates by various methods such as screen printing, ink jet printing, and spin coating [4]. Screen printing is extensively used to fabricate LEDs, LCDs, touch screens, chemical and biochemical sensors, and solar cells [31]. 
The two main categories of screen printing are flat-bed screen printing and rotary screen printing. These methods are shown schematically in Figure 1. 2. There are several advantages of flat-bed screen printing which make it suitable for many applications. For instance, it allows a large printing area, has low cost, and allows easy readjustment between printings. Screen printing is simple, adaptable to several kinds of organic inks and allows printing under ambient conditions. All these advantages provide low cost and a reproducible printing process on flexible substrates [32].

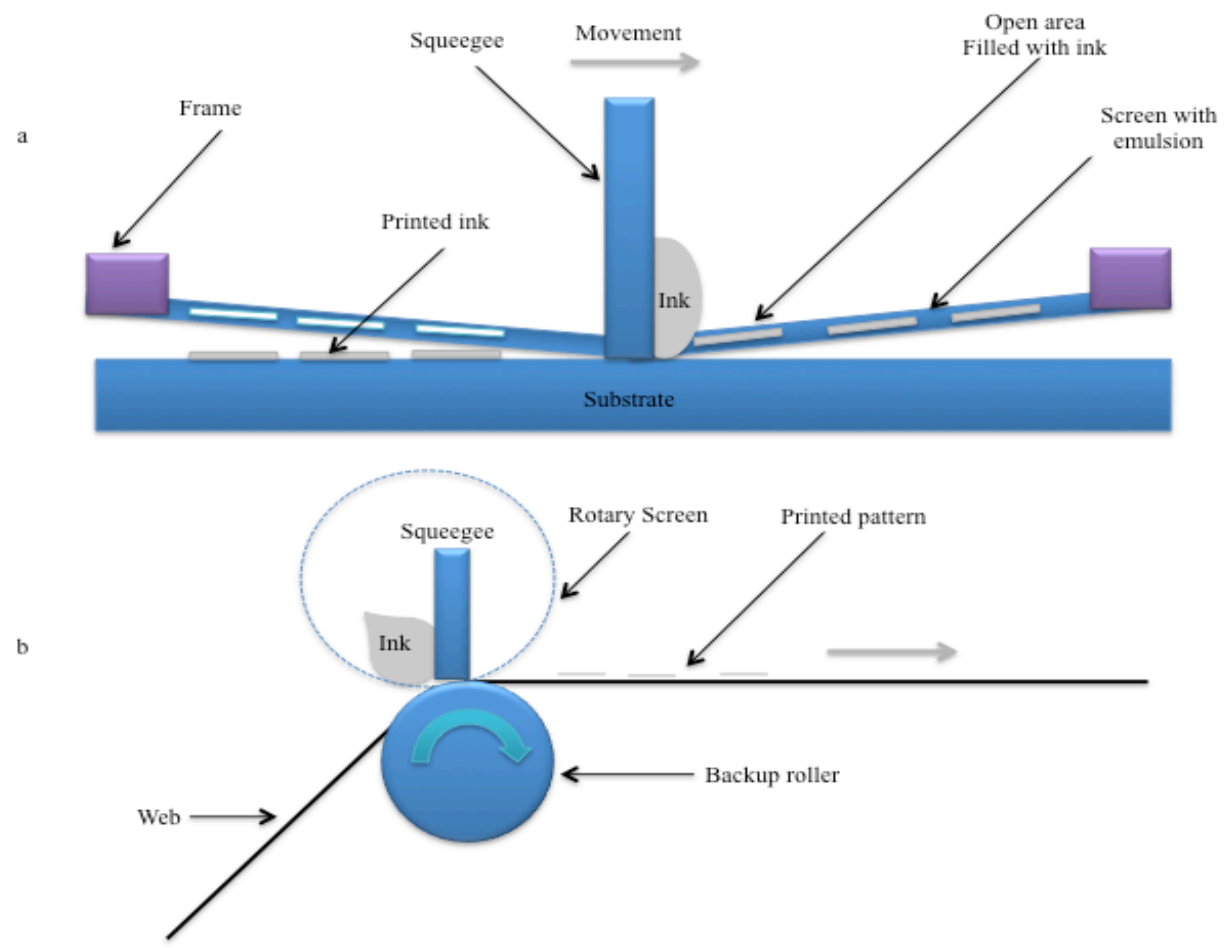

Figure 1. 2: Schematic illustration of screen printing; (a) flat-bed screen printing, (b) rotary screen printing.

In screen printing, the ink is forced through a patterned screen onto a substrate. Strong shear thinning behavior permits charging of the ink at low shear onto the screen, followed by printing through the screen at high shear. Recovery of the low-shear viscosity after printing is necessary to obtain sharp line definition. Hence, suitable inks for screen printing exhibit thixotropic rheological behavior with both time dependence and shear thinning. While rapid viscosity 
recovery promotes sharp line definition, too rapid recovery inhibits void filling and film leveling $[25]$.

\subsection{Rheological Behavior of Suspensions}

\subsubsection{Colloids and Suspensions}

Colloids are made up of sufficiently small particles in a suspending medium that the thermal forces affect their motion. They don't easily sediment and cannot be separated by a membrane. On the other hand, suspensions are a mixture in which the particle size is larger than the colloidal size [33].

\subsubsection{Principles of Rheology}

Figure 1. 3 shows a typical flow profile in a rotational rheometer, which is a simple liquid flow between two plates with distance h. The bottom plate is stationary, and the top plate is moving with velocity $\mathrm{V}$.

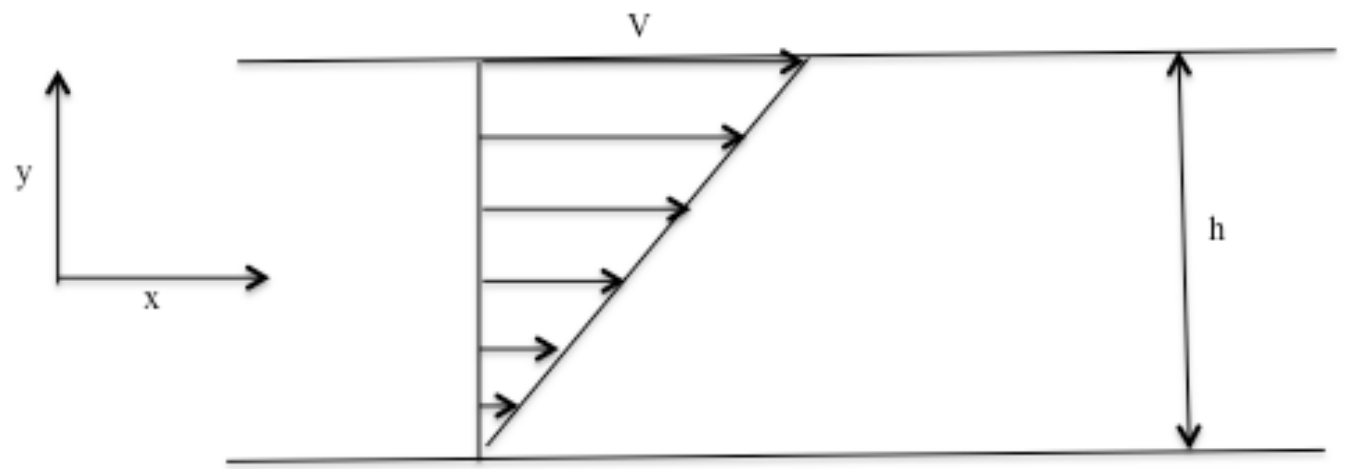

Figure 1. 3: Shear flow in typical rotational rheometer.

The shear rate can be defined by equation 1.3. 


$$
\frac{\mathrm{dv} v_{\mathrm{x}}}{\mathrm{dy}}=\frac{\mathrm{v}}{\mathrm{d}}=\dot{\gamma}=\text { constant }
$$

where $\frac{d v_{x}}{d y}$ is the gradient of velocity $V$ in the direction of $x, d$ is the distance between the plates, and $\dot{\gamma}$ is the shear rate.

Based on Newton's constitutive equation, the stress is proportional to the gradient of velocity. The proportionality constant is the viscosity $\eta$, which represents the resistance against the flow in Newtonian fluids according to equation 1.4.

$$
\sigma_{x y}=\eta \frac{d v_{x}}{d y}
$$

where, $\sigma_{\mathrm{xy}}$ is the shear stress that is the force per unit area. Newton's rheological constitutive equation is the simplest one [33].

Most suspensions are non-Newtonian fluids, and don't follow Newton's law, so that the shear stress is not directly proportional to the shear rate. Figure 1. 4 shows plots of shear stress versus shear rate for several types of non-Newtonian fluids [33]. 


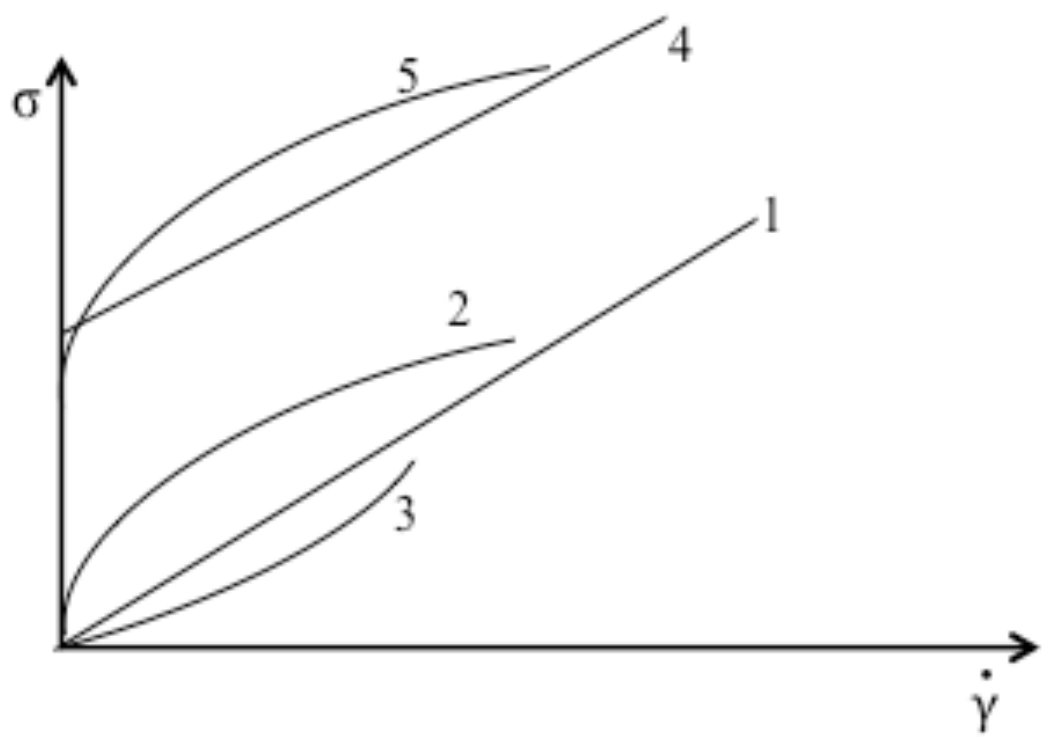

Figure 1. 4: General curves of shear stress versus shear rate: (1) Newtonian, (2) shear thinning, (3) shear thickening, (4), (5) material with yield stress [33].

The relation between shear stress and shear rate in shear thinning and shear thickening fluids can be expressed by a power law, which is shown in equation 1.5 .

$$
\sigma=\mathrm{k} \dot{\gamma}^{\mathrm{n}}
$$

where $\mathrm{n}$ is the power law index. $\mathrm{n}<<1$ represents shear thinning behavior, and $\mathrm{n}>1$ represents shear thickening behavior [33].

Viscoelastic materials exhibit the properties of elastic solids and viscous fluids in a combined manner. Elastic materials return to their original non-deformed state after the stress is removed. Hence elastic materials store the potential energy of the work done in deformation, and this energy can be recovered when they return to the original state. On the other hand, fluids have no memory, and when the stress is removed they remain in their last state. When particles are dispersed in a suspending medium, viscoelastic behavior may become important due to the presence of the particles. Oscillatory shear tests provide a good tool to investigate this elastic 
behavior in colloidal dispersions. Hence if a sinusoidal motion is applied to the parallel plate instead of a steady velocity according to the equation 1.6, a sinusoidal deformation according to equation 1.7 will be generated.

$$
\begin{gathered}
x_{p}(t)=x_{p, 0} \sin \omega t \\
\gamma(t)=\frac{x_{p, 0}}{d} \sin \omega t=\gamma_{0} \sin \omega t
\end{gathered}
$$

where, $\mathrm{x}_{\mathrm{p}, 0}$ is the peak displacement. The velocity of the top plate can be expressed by equation 1.8

$$
V(t)=\frac{d x_{p}}{d t}=x_{p, 0} \omega \cos \omega t
$$

The equation 1.9 can be driven from equation 1.7

$$
\dot{\gamma}(\mathrm{t})=\gamma_{0} \omega \cos \omega \mathrm{t}
$$

Based on Hooke's law as shown in equation 1.10, the shear stress $\sigma$ is proportional to the shear strain for ideal elastic materials.

$$
\sigma=\mathrm{G} \gamma
$$

where, G is the shear modulus. This stress is in phase with the strain. Substitution of equation 1.9 in Newton's law provides an equation for the oscillatory stress, which is shown in equation 1.11 


$$
\sigma(t)=\eta \gamma_{0} \omega \cos \omega t=\eta \gamma_{0} \omega \sin (\omega t+\pi / 2) .
$$

Equation 1.11 shows that the stress is shifted by $\pi / 2$ with respect to strain. The relative viscous behavior and elastic behavior can be measured through a phase difference between stress and strain in an oscillatory flow. The stress and strain versus time are shown in Figure 1. 5, and the phase difference for the stress in elastic, viscous, and viscoelastic materials is shown in Figure 1. 6.

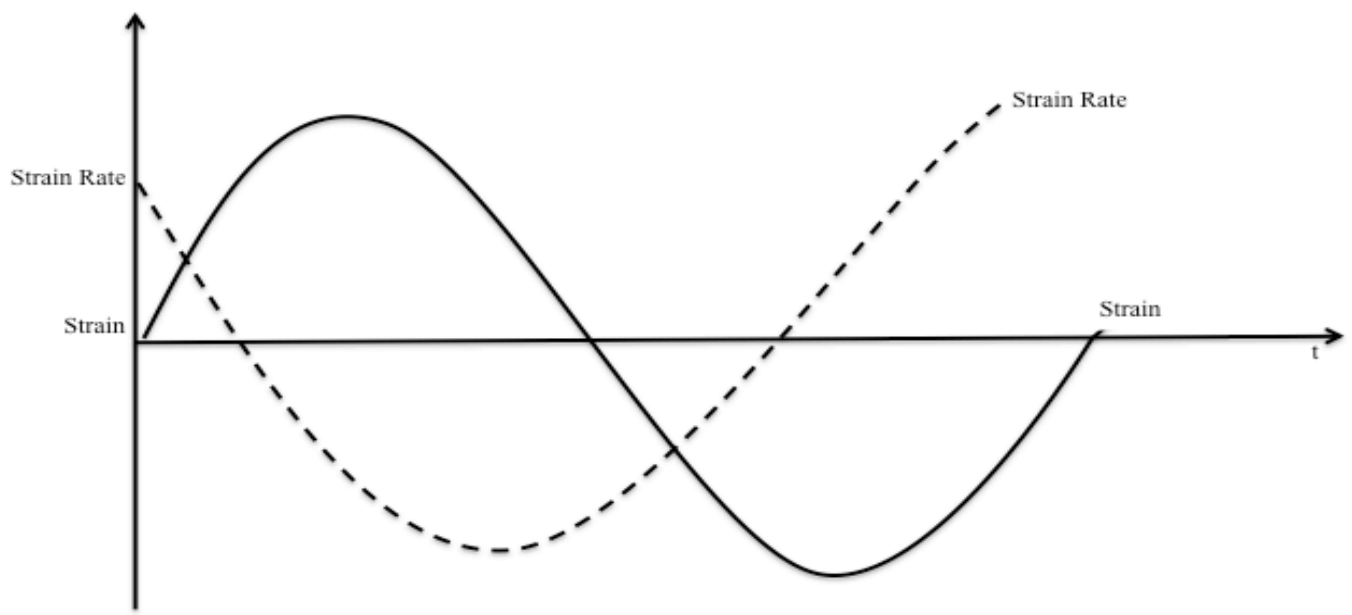

Figure 1. 5: Strain and shear rate illustration in an oscillatory flow.

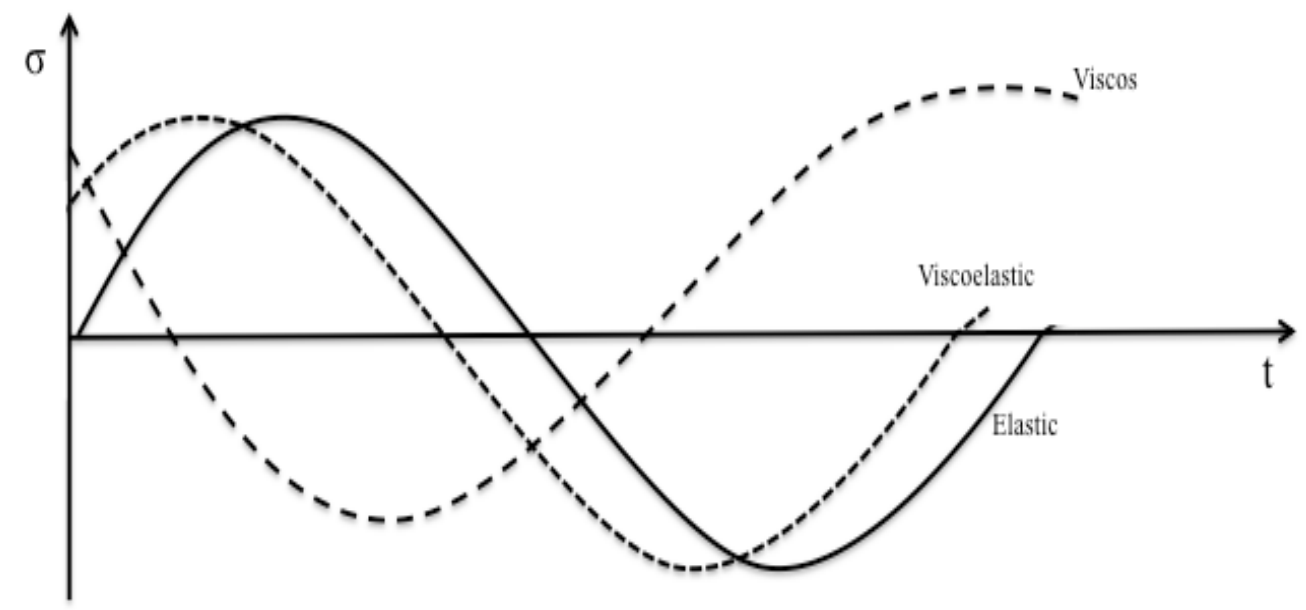

Figure 1. 6: Stress illustration in an oscillatory flow. 
The behavior of viscoelastic materials in oscillatory flow can be expressed in a form of Hooke's law, using a constant shear modulus G. This modulus can be decomposed into two parts as G' and $\mathrm{G}^{\prime \prime}$ and usually expressed in complex notation. $\mathrm{G}^{\prime}$, the real part, which is in-phase is known as storage modulus describes the elastic behavior. $G^{\prime \prime}$, the imaginary part, which is out-of-phase is known as loss or viscous modulus describes the viscous behavior

$$
\sigma=\left(\mathrm{G}^{\prime}+\mathrm{iG}^{\prime \prime}\right) \gamma
$$

In elastic materials, all of the energy is stored as potential energy, and is recovered when deformation is removed. In viscous materials all energy for the deformation is lost as heat. The loss angle or phase angle difference between stress and strain, as shown in equation 1.13, represents how much of the mechanical energy is lost as heat after deformation [33]

$$
\tan \delta=\frac{\mathrm{G}^{\prime \prime}}{\mathrm{G}^{\prime}}
$$

\subsubsection{Principles of Rheology in Colloidal Dispersions}

In colloidal dispersion systems, such as AgNW suspensions, solid particles are dispersed in a deformable fluid. During shear flow, the fluid is deformed but the particles are not. Rheology concepts can be applied to these kinds of dispersions by consideration of the heterogeneous nature of the system. The shear rates and stresses obtained from rheological measurements are the average of the local shear rates and stresses, and practically, the heterogeneous system is replaced by a pseudo-continuum system [33]. 


\subsubsection{Rheometry of Suspensions}

The investigation of the rheological behavior of suspensions can be challenging due to the necessity for an appropriate rheometer configuration, measurement protocol and data analysis suitable for suspensions [25]. Rheometer instruments can be categorized into two different types. In the first type, the sample is introduced through a cylinder or channel by assistance of pressure. In the second one, the motion of the one moving plate or wall drags the sample along. The rotational rheometer, which is the most prevalent rheometer for suspensions, works based on drag flow. There are different rotational rheometer systems such as cone and plate, parallel disk, and coaxial cylinders that are shown schematically in Figure 1. 7 [33].
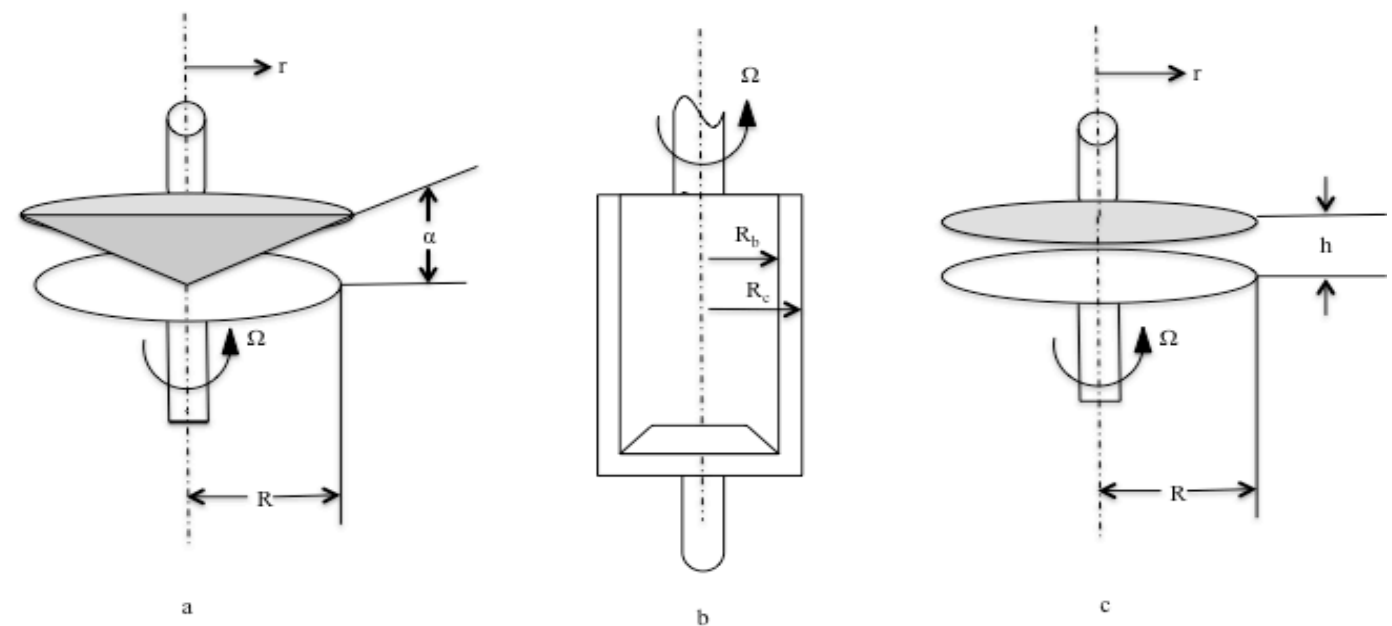

Figure 1. 7: Schematics of the rotational rhemeters; a: cone and plate, b: coaxial cylinder, c: parallel disk [33].

A parallel plate is more appropriate rather than a cone and plate configuration for silver nanowire suspensions because the finite size of the nanowires would complicate behavior near the apex of the cone [25].

In parallel disk rotational rheometers, which are also known as parallel plates, under the laminar flow assumption, the shear rate increases linearly with radial position from zero at the center to a maximum value at the edge according to the equation 1.14. 


$$
\dot{\gamma}(\mathrm{r})=\frac{\mathrm{v}_{\theta}(\mathrm{r})}{\mathrm{h}}=\frac{\Omega \mathrm{r}}{\mathrm{h}},
$$

where $\Omega$ is the rotational speed in $\mathrm{rad} / \mathrm{s}, \mathrm{r}$ is the radial distance, and $\mathrm{h}$ is the gap distance. Hence, the shear stress varies with radial position [33].

Because this configuration results in variation of shear rate with position, data analysis for the suspensions such as AgNWs suspensions should be based on comparison of results in experiments that differ only in particle composition [25].

\subsubsection{Concepts of Thixotropy and Shear Thinning Behavior}

The definition of thixotropy is the reduction in viscosity with time through the application of flow to a sample that has been at rest before, and the consequent viscosity recovery when the flow is removed. Although thixotropic systems also show shear thinning or shear thickening behavior, which is a viscosity change in response to the shear rate or shear stress, they have distinct meanings [33]. Shear thinning behavior is a viscosity reduction in response to an applied increasing shear rate, and shear thickening behavior is the increase in viscosity in response to an increase in applied shear rate. Suitable inks for screen printing exhibit thixotropic rheological behavior with both time dependence and shear thinning [25].

\subsubsection{Rheological Measurements}

Various rheological measurements might be used in order to investigate the rheological behavior of the particle suspensions such as silver nanowire conductive inks. These include Stress Sweep (SS), Steady State Flow (SSF), Frequency Sweep (FS), and Peak Hold (PH) measurements [25]. 
In most cases, the viscoelastic material's rheological behavior is independent of stress up to a specific stress value. Above this value, the viscoelastic modulus doesn't show linear behavior, and the moduli change in response to increasing stress. The variation and dependence of the storage modulus $\left(G^{\prime}\right)$, and viscous or loss modulus $\left(G^{\prime \prime}\right)$ on the applied stress provides information on the viscoelastic behavior of the fluid [34]. A SS measurement determines the Linear Visco-Elastic Region (LVR) with a plot of $\mathrm{G}^{\prime}(\mathrm{Pa})$ and $\mathrm{G}^{\prime \prime}(\mathrm{Pa})$ against stress (Pa). The SS measurement can be used to investigate further the relative magnitude of $G^{\prime}$ and $G^{\prime \prime}$ in response to increasing stress [25].

Thixotropic materials lose structure in the shearing step, and rebuilt it after the shear is removed [34]. A SSF measurement can be used to probe the shear thinning behavior of the sample through the plots of viscosity (Pa.s) versus shear rate (1/s). [25].

After the LVR determination, the sample can be further characterized by a FS experiment at a stress bellow the critical stress to determine the variation of $\mathrm{G}^{\prime}$ and $\mathrm{G}^{\prime \prime}$ as functions of angular frequency. The FS measurement provides more information about the effect of colloidal forces, and the interactions between particles [25].

PH measurements can provide information regarding the viscosity versus time at constant shear rate value by holding the shear constant on sample at defined intervals [25]. These kinds of measurements can be used to mimic the rheological behavior of the ink during the screen printing.

\subsection{Build-up Ink Structure and Governing Equations}

Recovery of the low-shear viscosity after printing is necessary to obtain sharp line definition. While rapid viscosity recovery promotes sharp line definition, too rapid recovery inhibits void filling and film leveling $[4,5,25]$. Hence, the time-scale for viscosity recovery and build-up 
structure after printing play an important role in obtaining the best line definition and film leveling [35]. The Stretched Exponential model (SEmo) has been suggested as an appropriate model in order to correlate and predict the structural build-up in suspensions such as conductive inks $[35,36]$.

The rheological properties of water-based silver nanowire conductive ink are complex and challenging due to the necessity for an appropriate rheometer configuration, measurement protocol and data analysis suitable for suspensions $[25,37]$. In these kinds of systems different forces and interactions play a crucial role in the rheological behavior. Based on the domination of the different forces and interactions, the silver nanowires can form different structures, which determine the rheological characteristics of the ink during different steps of screen printing [37]. This behavior is controlled by the balance among Brownian motion, thermodynamic interactions and hydrodynamic interactions. Specifically, the rheological behavior of silver nanowire ink is controlled by the alignment and orientation of the nanowires with respect to the flow. Brownian motion tends to randomize orientation. Hydrodynamic interactions tend to align wires with respect to the flow. At low shear rate, the random orientation of the wires increases the viscosity. During shearing the nanowires orient in the direction of the flow decreasing the viscosity. After the shear is reduced, recovery of viscosity requires time. Thermodynamic interactions include attractions or repulsions between wires arising for example from the interaction of double layers or direct metal to metal contact.

At higher silver nanowire content, the Brownian motion during low shear rate and flow orientation during shearing are higher, and the viscosity is higher [8,37]. The rotational Peclet number $(\mathrm{Pe})$, which is the ratio of applied shear rate to the rotational diffusion coefficient, can be used to assess the dominance of Brownian motion compared to non Brownian motion [38, 39]. 
The dominance of different interactions strongly depends on the AgNWs content of the suspension, which are classified as a dilute, semi dilute, and concentrated. The transition between dilute, semi dilute, and concentrated dispersion can be determined based on the number density of suspension, diameter, and length of the AgNWs [40].

Sometimes there are various structural change mechanisms, especially in the concentrated regime, and the contribution and quantity of each mechanism are not precisely known. In this situation, the behavior can be quantified in terms of activation energy [41]. A step change in shear rate is followed by a time dependent transition from one steady shear stress to another because the change in suspension structure is a rate process. In moving from a high shear to a low shear state, the more oriented arrangement of wires changes to a more random arrangement. This transition is referred to as build-up. In the peak hold $(\mathrm{PH})$ test a low shear rate is applied initially, the shear is then stepped to a high rate and finally stepped back to the low rate, mimicking the sequence of ink charging, through-screen printing, and film leveling in screen printing. The time dependence of the transition from high to low shear can be correlated with the Stretched Exponential model (SEmo), expressed in the equation $1.15[35,36]$.

$$
\eta=\eta_{0}+\left(\eta_{\infty}-\eta_{0}\right)\left(1-e^{-(t / \tau)^{\varepsilon}}\right)
$$

where $\eta_{0}$ is the steady viscosity at high shear rate, $\eta_{\infty}$ is the steady viscosity at low shear rate, $\tau$ is the characteristic time for the transition, and $\varepsilon$ is a dimensionless constant that can be assumed to be unity in the simplest case. The values of $\eta_{0}$ and $\eta_{\infty}$ are obtained from the PH test. The time constant $\tau$ is found by fitting to the SEmo equation $[35,36]$. 
The dependence of characteristic time on temperature can be expressed in form of the Arrhenius equation as is shown in equation 1.16 .

$$
\frac{1}{\tau}(\mathrm{T})=\frac{1}{\tau_{0}} \mathrm{e}^{-\frac{\mathrm{E}_{\mathrm{a}}}{\mathrm{RT}}}
$$

where $\frac{1}{\tau_{0}}$ is the frequency factor, $E_{a}$ is the activation energy, $R$ is the universal gas constant, and $\mathrm{T}$ is the absolute temperature. A semi-log plot of the rate constant versus the reciprocal temperature provides the parameters.

$$
\ln \left(\frac{1}{\tau}\right)=\ln \left(\frac{1}{\tau_{0}}\right)-\frac{E_{a}}{R}\left(\frac{1}{T}\right)
$$

the slope of the natural $\log$ of the $\frac{1}{\tau}$ versus $\frac{1}{T}$ plot gives $-\frac{E_{a}}{R}$.

In dilute suspensions of AgNWs, the wires can rotate easily without any interference from the other wires, and the wire diffusivity is controlled by Brownian motion. In semi dilute suspensions, there are particle interactions between neighboring wires that inhibit free rotation. In concentrated suspensions of AgNWs, the wire dimensions are large compared to the spacing between wires, and the volume of interaction should be considered [40]. The transition from dilute to semi dilute suspensions can be evaluated through a dimensionless constant defined by equation 1.18.

$$
u L^{3}=\beta,
$$


where $v$ is the number density (the numbers of wires per unit volume), $L$ is the wire length, and $\beta$ is a dimensionless constant. A $\beta$ value of less than 30 corresponds to the dilute regime [40].

The value of $\frac{1}{\mathrm{dL}^{2}}$ can be used to identify the transition to the concentrated regime ( $\mathrm{d}$ is the wire diameter). In the concentrated AgNWs suspension, $v>\frac{1}{\mathrm{dL}^{2}}$. At higher concentration of AgNWs $\left(v>\frac{4.2}{\mathrm{dL}^{2}}\right)$, the wires can orient into a nematic regime [40].

The transition from Brownian dominance to non-Brownian dominance can be evaluated by a rotational Peclet number (or Weissenberg number) as shown in equation 1.19.

$$
\mathrm{Pe}=\frac{\dot{\gamma}}{\mathrm{D}_{\mathrm{r}}}
$$

where $\mathrm{D}_{\mathrm{r}}$ is rotational diffusion coefficient, and $\dot{\gamma}$ is the shear rate. For wire-like structures, the rotational diffusion coefficient can be estimated by equation 1.20 [38].

$$
\mathrm{D}_{\mathrm{r}}=\frac{\delta \mathrm{K}_{\mathrm{b}} \mathrm{T} \ln \left(\mathrm{a}_{\mathrm{r}}\right)}{3 \pi \eta \mathrm{L}^{3}\left(\mathrm{vL}^{3}\right)^{2}}
$$

where $\delta$ is unity, $\mathrm{K}_{\mathrm{b}}$ is the Boltzmann constant, $\mathrm{a}_{\mathrm{r}}$ is the aspect ratio of AgNWs, $v$ is the number density of AgNWs, and L is the length of the AgNWs [38]. Very large Peclet number (Pe $>>1$ ) predicts non-Brownian motion during the viscosity recovery process after screen printing, and low Peclet number $(\mathrm{Pe}<<1)$ predicts Brownian motion $[38,40]$. 
A simple way to probe the non-Brownian mechanism or thermodynamic interactions is to measure the activation energy of flow [41]. The dependence of viscosity on temperature can be expressed by equation 1.21 .

$$
\eta=K^{\prime} \dot{\gamma}^{n-1} e^{\left(\frac{\Delta E_{a}}{R T}\right)}
$$

where $\mathrm{K}^{\prime}$ is constant, $\dot{\gamma}$ is shear rate, $\mathrm{n}$ is the power law exponent, and $\Delta \mathrm{E}_{a}$ is the change in activation energy. The activation energy can be expressed as activation enthalpy $(\Delta \mathrm{H})$, and activation entropy $(\Delta \mathrm{S})$ according to equation $1.22[41]$

$$
\Delta \mathrm{E}_{\mathrm{a}}=\Delta \mathrm{H}-\mathrm{T} \Delta \mathrm{S} .
$$

The temperature dependence of the low viscosity at high shear $\left(\eta_{I}\right)$ corresponds to the viscosity at the end of the second interval in the PH test, and the viscosity at the end of recovery process $\left(\eta_{R}\right)$ corresponds to the viscosity at the end of third interval in the PH test can be written

$$
\begin{aligned}
& \eta_{I}=K^{\prime} \dot{\gamma}^{\mathrm{n}-1} \mathrm{e}^{\left(\frac{\Delta \mathrm{H}_{\mathrm{I}}-\mathrm{T} \Delta \mathrm{S}_{\mathrm{I}}}{\mathrm{RT}}\right)}, \\
& \eta_{\mathrm{R}}=\mathrm{K}^{\prime} \dot{\gamma}^{\mathrm{n}-1} \mathrm{e}^{\left(\frac{\Delta \mathrm{H}_{\mathrm{R}}-\mathrm{T} \Delta \mathrm{S}_{\mathrm{R}}}{\mathrm{RT}}\right)} .
\end{aligned}
$$

Plots of the natural $\log$ of $\eta_{I}$, and $\eta_{R}$ versus $\frac{1}{T}$ provide the activation enthalpies, 


$$
\begin{aligned}
\ln \eta_{\mathrm{I}} & =\frac{\Delta \mathrm{H}_{\mathrm{I}}}{\mathrm{R}}\left(\frac{1}{\mathrm{~T}}\right)+\left[\ln \left(\mathrm{K}^{\prime} \dot{\gamma}^{\mathrm{n}-1}\right)-\frac{\Delta \mathrm{S}_{\mathrm{I}}}{\mathrm{R}}\right], \\
\ln \eta_{\mathrm{R}} & =\frac{\Delta \mathrm{H}_{\mathrm{R}}}{\mathrm{R}}\left(\frac{1}{\mathrm{~T}}\right)+\left[\ln \left(\mathrm{K}^{\prime} \dot{\gamma}^{\mathrm{n}-1}\right)-\frac{\Delta \mathrm{S}_{\mathrm{R}}}{\mathrm{R}}\right],
\end{aligned}
$$

the slopes of give $\frac{\Delta H_{I}}{R}$, and $\frac{\Delta H_{R}}{R}$, respectively.

\subsection{Electrical Characteristics of Conductive Inks and Printed Patterns}

Electrical resistivity or specific electrical resistance is a measure of the materials capacity to carry electrical current. The electrical resistivity of a thin line can be calculated by equation 1.27.

$$
\rho=\frac{\text { ER. A }}{L}=\frac{\text { ER. } w \cdot \text { th }}{L}
$$

where $\rho$ is the electrical resistivity in $\Omega \mathrm{m}$, ER is the electrical resistance in $\Omega$ defined by Ohm's law, $A$ is the cross section area in $\mathrm{m}^{2}, \mathrm{~L}$ is the length of line in $\mathrm{m}, \mathrm{w}$ is width in $\mathrm{m}$, and th is the thickness of the line in $\mathrm{m}$.

For a conductive film with homogenous electrical resistivity, a sheet resistance $\left(E_{\mathrm{s}}\right)$ can be defined based on equation 1.28

$$
\mathrm{ER}_{\mathrm{s}}=\frac{\rho}{\mathrm{th}}
$$

The electrical conductivity of the conductive inks increases with increasing particle content. Hence, when conductive particle content is increased, the number of paths per unit length of the printed pattern is increased as well [4]. 
Charge transport through the AgNW inks consists of two parts, conduction along the wires and transfer across the junctions. Longer and thinner AgNWs increase the optical transmittance while the sheet resistance is constant. Thinner and longer nanowires decrease the percolation threshold in electrical conductance. Both the diffusive transmittance and specular transmittance are important for transparent electrodes. In the case of AgNWs electrodes, the diffusive transmittance is larger than the specular transmittance. The large difference between these two transmittances leads to haze, which is problematic for some devices such as touch screens. At the same time, this difference can be advantageous for devices in which light scattering is favorable such as solar cells where it produces efficiency [18].

Shorter AgNWs have higher transparency, and longer AgNWs have lower sheet resistance. Hence, an improvement in the AgNWs conductive film performance can be achieved by utilizing AgNWs with high aspect ratio and small diameter [42].

AgNWs based transparent flexible films, which have similar optical properties to ITO-based films, can be utilized in solution at ambient temperatures. The sheet resistance of the electrode is proportional to the density of AgNWs for a particular area. This density should much be higher than the percolation threshold to attain low sheet resistance without changing optical transparency. Application of longer AgNWs can cause low sheet resistance without changing optical transparency. The critical density of AgNWs in a random network can be calculated by equation 1.29.

$$
\mathrm{L} \sqrt{\pi N_{c}}=4.326 \text {, }
$$


where $\mathrm{N}_{\mathrm{c}}$ is the critical density of AgNWs, and L is the length of AgNWs. The AgNWs diameter also plays an essential role in the optical characteristics of the fabricated films. Smaller diameters produce low haze and high transmittance [20].

The electrical properties of AgNWs films can be explained through the percolating network theory, which includes the two essential parameters percolation concentration and conductivity critical exponent. One of the percolation relations that can be used for sheet resistance calculation is shown in equation 1.30 .

$$
\mathrm{ER}_{\mathrm{s}} \sim \frac{\mathrm{M}}{\left(\mathrm{N}_{\mathrm{s}}-\mathrm{N}_{\mathrm{c}}\right)^{\mathrm{t}}},
$$

Where $\mathrm{M}$ is the material constant, $\mathrm{N}_{\mathrm{c}}$ is the critical percolation concentration, $\mathrm{N}_{\mathrm{s}}$ is the number of wires per area, and $t$ is the critical exponent [21]. Based on percolation theory, larger length or lower diameter corresponding to larger aspect ratio of $\mathrm{AgNWs}$ causes to transparent electrodes owing better performance [22]. 


\section{CHAPTER 2}

\section{LITERATURE REVIEW}

\subsection{Synthesis and Characterization of Silver Nanowires}

A number of investigators have studied the polyol process for AgNW synthesis over the last ten years. This work provides reliable recipes for synthesis as well as some mechanistic insight. A general understanding that would allow full control of wire dimensions is still lacking.

Korte et al. investigated rapid $\mathrm{AgNWs}$ synthesis through a polyol process with $\mathrm{CuCl}$ or $\mathrm{CuCl}_{2}$ as salt mediator. They showed that $\mathrm{CuCl}$ or $\mathrm{CuCl}_{2}$ in presence of PVP could assist the growth and formation of AgNWs. They reported that chloride must be present to reduce the initial level of $\mathrm{Ag}^{+}$, and the cations are required to scavenge atomic oxygen. They used SEM and TEM to investigate the morphology of Ag seeds and the synthesized AgNWs [1].

Lee et al. reported large scale synthesis of very long AgNWs through a Successive Multiple Growth (SMG) mechanism, in which AgNWs from the first step are used as seeds to produce very long AgNWs in the second step. They synthesized AgNWs with average length of $120 \mu \mathrm{m}$ and a maximum length of 400-500 $\mu \mathrm{m}$. They used TEM, SEM, and HRTEM to investigate the morphology, length, and diameter of the AgNWs. A thermal stability analysis of the AgNWs was done by Differential Scanning Calorimetry (DSC), and Thermo Gravimetric Analysis (TGA) $[2]$.

Sun et al. synthesized AgNWs with the lateral dimensions of 30-40 nm, and length up to $50 \mu \mathrm{m}$ in a large scale batch process. They used platinum nanoparticles as seeds for nucleation and 
growth of AgNWs in presence of PVP. SEM and TEM were utilized to investigate the morphology of the as-synthesized samples, and synthesized AgNWs [10].

Mao et al. synthesized AgNWs with different aspect ratios through a self-seeding polyol process in the presence of PVP, which acted as both structure capping agent, and protecting agent. They proposed a growth mechanism based on formation of five-twinned silver nanoparticles as seeds for further growth of the particles in a wire shape. PVP adsorption on $\{100\}$ facets through the Ag-O bonding facilitate elongated growth. Field Emission Scanning Electron Microscopy (FESEM) was used to investigate the microstructure of the synthesized AgNWs. X-ray Diffraction (XRD), Fourier Transform Infrared Spectroscopy (FTIR), X-ray Photoelectron Spectroscopy (XPS), and Thermo Gravimetry-Differential Scanning Calorimetry (TG-DSC) were used to characterize the AgNWs, and the effect of the different steps of the separation after synthesis [16].

Chen et al. synthesized AgNWs with diameters in the range of 20 to $500 \mathrm{~nm}$ by addition of control agents in the form of molecules, metal cations, and inorganic anions into the regular polyol process. They proposed that these control agents affect the morphology and diameter of the AgNWs by regulating the rate of formation of silver seeds. They used SEM, TEM, and XRD to observe the effect of control agents including $\mathrm{KOH}, \mathrm{KBr}, \mathrm{KCl}, \mathrm{H} 2 \mathrm{O}, \mathrm{Fe}\left(\mathrm{NO}_{3}\right)_{3}, \mathrm{pdCl}_{2}, \mathrm{Na}_{2} \mathrm{~S}$, and ascorbic acid on the morphology and yield of synthesized AgNWs. They showed the diameter dependence of Surface Enhanced Raman Scattering (SERS), which illustrates the potential application of AgNWs with controlled diameter in optical nanodevice fabrication [9].

Schustte et al. used $\mathrm{AgCl}$ nanocubes with various dimensions as heterogeneous initial nuclei. They showed that the diameter of the synthesized AgNWs was independent of the dimensions of the $\mathrm{AgCl}$ nanocubes. They concluded that wire diameter is independent of the nucleation event. 
Consequently, they proposed that wire growth is limited by the reduction rate of $\mathrm{Ag}^{+}$through a surface catalyzed reduction reaction [43].

Yan et al. reported a water-involved synthesis of AgNWs through a scalable and rapid heterogeneous nucleation process. They proposed that the addition of small amounts of water with an optimal ratio to the reducing agent of $0.25 \%$ plays an important role in obtaining a high yield of AgNWs. They synthesized AgNWs with diameters of 60-90 nm and lengths of 8-12 $\mu \mathrm{m}$ with high yield [17].

Hunyadi Murph et al. proposed an orientated attached-growth mechanism for AgNWs synthesis, in which the initial structure of the seeds has an effect on the final organization of the crystals. They emphasized the importance of interactions between neighboring nanoparticles such as van der Waals, Coulombic, and dipolar interactions in this mechanism that are affected by type of solvent, solution condition, $\mathrm{pH}$, and dielectric constant [24].

Lim et al. reported high yield synthesis of AgNWs by a heated up (heating from room temperature to desired one at a specific heating rate) polyol process. They synthesized AgNWs with an average length of $20 \mu \mathrm{m}$. They observed that the heat-up method could facilitate the formation of initially Ag seeds and their growth to the wire structure compare to the hot-injection polyol method [44].

Gottesman et al. synthesized AgNWs and AgNPs in a millifluidic reactor through the polyol process at high temperature of $198{ }^{\circ} \mathrm{C}$. They suggested the feasibility of optimizing the reaction conditions of the flow in order to synthesize high yield of AgNWs in continuous flow in a short time [45]. 


\subsection{Sensitivity Analysis and Parametric Study}

To optimize the process, the desired outcome must be defined. The important variables here are yield and morphology. In addition, the important process variables must be identified, and their effect on outcome must be assessed.

Nghia et al. synthesized AgNWs through a polyol process using microwaves. They found that the yield, dimension, and morphology of the synthesized AgNWs strongly depend on the concentration of the PVP stabilizer, the concentration of $\mathrm{NaCl}$ and $\mathrm{AgNO}_{3}$, and the heating time. They concluded that intermediate concentration of $\mathrm{NaCl}(3 \mathrm{mM})$, low concentration of PVP (50 $\mathrm{mM}$ ), and 3 min heating time are advantageous for AgNWs synthesis [46].

Song et al. investigated the effect of PVP concentration and molecular weight on the morphology and yield of synthesized AgNWs. They proposed that adsorption and steric effects are responsible for the effect of PVP on the morphology of the Ag nanocrystals. They suggested that when the PVP MW is low selective adsorption of PVP on $\{100\}$ facets is dominant, whereas at higher MW chemical adsorption is dominant and adsorption is distributed on all facets. They also concluded that the MW of PVP is more critical than concentration [47].

Johan et al. proposed a growth mechanism of the AgNWs polyol process through various mediating agents such as $\mathrm{NaCl}$ and $\mathrm{CuCl}_{2}$. They concluded that the presence of both cation and anion are necessary for the formation of the AgNWs, They observed that in the absence of mediating agents the final products include particle as well as wire [27].

Lin et al. investigated the effect of $\mathrm{AgNO}_{3}$ concentration. The results showed that the concentration and injection rate of $\mathrm{AgNO}_{3}$ and the reaction temperature have a strong affect on the growth characteristics of AgNWs. They showed that with reduced $\mathrm{AgNO}_{3}$ concentration, the AgNWs diameter decreased, and consequently their aspect ratio increased. They concluded that 
AgNWs with high aspect ratio could be produced with a $\mathrm{PVP} / \mathrm{AgNO}_{3}$ concentration mol ratio of $16[48]$.

Coskun et al. did an extensive parametric study of AgNWs polyol synthesis. They investigated the effect of temperature, mol ratio of $\mathrm{PVP} / \mathrm{AgNO}_{3}$, rate of injection, rate of stirring and amount of $\mathrm{NaCl}$ as mediating agent on the morphology and yield. They concluded that anisotropic growth is favored by increasing reaction temperature with an optimum of $170{ }^{\circ} \mathrm{C}$. Based on their results both low and high injection rate cause the formation of nanostructures other than nanowires. They showed that low and high $\mathrm{PVP} / \mathrm{AgNO}_{3}$ ratios have the same effect as injection rate. They demonstrated the necessity of $\mathrm{NaCl}$ as mediating agent, with an optimum concentration of $12 \mu \mathrm{M}$ [49].

Lee et al. investigated the effect of $\mathrm{AgNO}_{3}$ sonication time on the morphology and yield, since AgNWs solution is prepared by sonication before it is injected into the reactor. They found that thorough sonication produced very small twinned particles of $\mathrm{AgNO}_{3}$ resulting in growth of very long AgNWs with high quality. They found that the optimum sonication time is $5-7$ min by which time the solution turns a transparent red [2].

Perez at al. investigated the polyol synthesis of Ag nanostructures statistically by Design of Experiment (DoE) procedures. They showed the simultaneous effects of the important parameters in the polyol process on the morphology of the synthesized Ag nanostructure rather than the separate effect of each parameter. They concluded that longer reaction time and higher temperature produced larger nanostructures [50].

\subsection{Silver Nanowire Based Conductive Inks and Adhesives for Electronic Media}

Some prior works on the application of silver flake, AgNPs, and AgNWs in conductive media are summarized in this section. 
Faddoul et al. formulated water-based silver inks suitable for screen printing with silver (mixture of flake and spherical) contents of $67 \%$ to $72.5 \%$ by weight. These pastes were printed onto Low Temperature Co-fired Ceramic (LTCC) with uniform edges lines, and showed electrical resistivities from 1.6 to $3.3 \times 10^{-8} \Omega \mathrm{m}$. They found that the best line definition is obtained with $70-72 \%$ silver paste [4].

$\mathrm{Hu}$ et al. used a Mayer rod technique to create conductive and transparent AgNWs electrodes. They used long and thin AgNWs to improve conductivity and optical transmittance. They found that the resistance of individual wires about $10 \mu \mathrm{m}$ in length is $200-300 \Omega$, and the resistance of wire-wire junctions is around $450 \Omega$. They achieved $8 \Omega / \mathrm{sq}$ and $80 \%$ diffusive transmittance, and $20 \Omega / \mathrm{sq}$ and $80 \%$ specular transmittance, which could be suitable for solar cell applications. They performed an environmental and physical stability test on the coated substrates, and proposed the application of long/thin AgNWs, pressing, and coating with gold to improve performance [18].

Liu et al. described the fabrication of uniform, transparent, and conductive silver films utilizing the rod-coating technique. They found that hydrogen chloride $(\mathrm{HCl})$ vapor incubation could decrease film resistance by destroying the surface oxide layer. They concluded that AgNWs would be a promising option in flexible electrode fabrication for solar cell applications [42].

Kumar et al. fabricated AgNW based flexible electrodes with high transparency, high conductivity, and low haze by controlling ink composition, aspect ratio of AgNWs, and conditions of processing. Films fabricated by the Mayer rod technique showed $\mathrm{R}_{\mathrm{s}} \leq 300 \Omega / \mathrm{sq}, \mathrm{T}$ $\geq 90 \%$, and Haze of $2 \%$. They concluded the application of these AgNW based films as replacement of ITO for display and solar cell applications is feasible [20]. 
Yang et al. investigated the shape effects of silver particles as conductive filler of conductive inks on electrical properties of inkjet-printed patterns. They synthesized Ag nanoparticles with diameters of about $64 \mathrm{~nm}, \mathrm{Ag}$ nanoplates with thicknesses of about $30 \mathrm{~nm}$ and lengths of 600 $\mathrm{nm}$, and Ag nanorods with lengths of about $5 \mu \mathrm{m}$ and diameters of about $100 \mathrm{~nm}$. They fabricated conductive patterns of around $54 \mathrm{wt} . \% \mathrm{Ag}$ nanorods and nanoparticles with resistivity of $\sim 3.2 \times 10^{-5} \Omega \mathrm{cm}$. The conductive patterns of $\sim 64 \mathrm{wt} . \% \mathrm{Ag}$ nanoplates and nanoparticles showed resistivity of $\sim 6.8 \times 10^{-5} \Omega \mathrm{cm}[19]$.

Park et al. formulated $\mathrm{Ag}$ nanoparticle inks of 80 wt.\% Ag nanoparticle, 19 wt.\% organic solvents, and $1 \mathrm{wt} . \% \mathrm{~Pb}$-free frit to screen print on alumina substrates. They investigated the conductivity of the inks as they depend on sintering temperature. They showed that nanoparticle densification would take place at a sintering temperature of $400^{\circ} \mathrm{C}$. They achieved the lowest electrical resistivity of $4.11 \mu \Omega \mathrm{cm}$ at $450^{\circ} \mathrm{C}[51]$.

Wang et al. prepared AgNW based conductive, transparent, and flexible films by a vacuumfiltration technique. They achieved $92 \%$ optical transmittance and $11 \Omega /$ sq surface resistivity in the fabricated films in which both sheet resistance and transmittance decrease with increasing AgNWs content. They showed the potential of AgNWs based films for electrical devices due to their high conductivity and transparency, flexibility, and scalable production [52].

Takehiro et al. synthesized AgNWs by a polyol process and applied them to transparent electrode fabrication. They used mechanical pressing instead of heat treatment to decrease sheet resistance, which exists because of weak AgNWs contact, and residual PVP on the wire surfaces. They showed the sheet resistance was reduced from $6.9 \times 10^{6} \Omega / \mathrm{sq}$ to $8.6 \Omega / \mathrm{sq}$ by mechanical pressing [53]. 
De et al. fabricated thin conductive and transparent AgNW based films. They showed that for film thicknesses less than around $160 \mathrm{~nm}$, the films function as 2D networks. At higher thicknesses they showed bulk-like behavior. They showed that the optical transmittance decreases with increasing film thickness, while the optical conductivity decreases to $6472 \mathrm{~S} / \mathrm{m}$ for a thickness of $550 \mathrm{~nm}$. They showed that fabrication of these films from low diameter AgNWs produces better performance and uniformity [14].

Madaria et al. fabricated AgNW based films with high conductivity and transparency on both flexible and rigid substrates through a Poly Dimethyl Siloxane (PDMS)-assisted transfer process and vacuum filtration. The uniform fabricated films had sheet resistance as low as $10 \Omega / \mathrm{sq}$ at $85 \%$ optical transparency. They showed that AgNW films created 2D percolation networks and had high conductivity even under bending, which would revert to low resistance when stress were removed [15].

Polavarapu et al. deposited AgNPs ink on plastic and glass substrates. They achieved high conductivity with very mild-temperature annealing because of the aggregation of AgNPs after solvent drying [29].

Samali et al. formulated a Low-Volatile Organic (LVO) compound ink adaptable for screen printing. Silver microparticles and silver flake were used as conductive filler and monofunctional liquid acrylate monomers as binder that acted as a conductive adhesive due to its thermoplastic nature. They printed ink on both flexible polysulfone, and rigid FR4 substrates. They achieved low resistivity of $1 \Omega$ for $\mathrm{Cu}$ Radio-Frequency Identification (RFID) antenna applications [31]. Liangbing et al. investigated different metal nanostructures such as nanowires, nanogrids, and nanofibers for transparent electrodes fabrication. They illustrated the promising applications of these electrodes especially for solar cells fabrication due to their high scattering effect [22]. 
Lee et al. synthesized very long AgNWs through a Successive Multistep Growth (SMG) process for conductive, transparent, and flexible AgNWs based touch panel fabrication. They combined the SMG synthesis method and laser nano-welding process for electrodes fabrication. They suggested the possibility of the development of portable and wearable electronics based on this knowledge in the future [54].

\subsection{Sintering of the Printed Conductive Patterns}

Best performance of conductive media is attained when the particles are bonded to one another as in sintering or interdiffusion.

Teng et al. synthesized AgNPs with average diameter of $90 \mathrm{~nm}$ by the polyol process. The wires were dispersed in deionized (DI) water and printed on a FR4 substrate by screen printing. They used saturated sodium chloride solution treatment to make the pattern conductive without sintering. The $\mathrm{NaCl}$ promoted coalescence of the AgNPs following PVP detachment. They decreased the electrical resistivity of patterns from $2.2 \times 10^{-5}$ to $9.91 \times 10^{-6} \Omega \mathrm{cm}$ by that method $[55]$.

Daisuke et al. formulated AgNPs ink that could be sintered at room temperature by solvent drying and evaporation, and they achieved a low resistivity of $4.9 \times 10^{-6} \Omega \mathrm{m}$. They emphasized the importance of the solvent as well as dispersant in ink [56].

Kwang-Seok et al. investigated the effect of sintering condition on the properties of screen printed silver patterns. They investigated the effect of sintering temperature, atmospheric conditions of sintering and rate of temperature increment. They showed that by adjusting these three parameters the electrical properties and density of the printed patterns could be controlled. They obtained the lowest electrical resistivity of $2.88 \mu \Omega \mathrm{cm}$ at $300{ }^{\circ} \mathrm{C}$ sintering temperature [57]. 


\subsection{Rheological Behavior and Activation Energy}

Faddoul et al. formulated a water-based silver ink and investigated the rheological behavior of the ink during simulated screen printing and during oscillatory (stress sweep) rheological tests. They achieved the highest viscosity of 320 Pa.s at low shear rate of $0.11 / \mathrm{s}$, but found that better patterns with sharper line definition would be achieved with lower viscosity less than 200 Pa.s. They achieved optimal patterns and sharp line definition for ink composed of 70-72\% silver by weight. They also investigated the ink behavior based on Hershel-Bulkley model to measure the yield stress, and they concluded that the ink of $75 \%$ silver would require a stress as high as 501 $\mathrm{Pa}$ to flow through the screen. They found strong dependency of line uniformity on rheological behavior of the ink during the printing process and on the plastic and elastic moduli of the ink. They showed that the ratio of viscous or loss modulus $\left(G^{\prime \prime}\right)$ to elastic or storage modulus $\left(G^{\prime}\right)$ decreases with increasing silver content producing a thicker ink [4].

In another study, Faddoul et al. investigated the effect of silver content of $70-80 \%$ on the electrical and rheological behavior of the silver inks. They showed that water-based formulated inks had similar quality to commercial silver inks of more than $75 \%$ silver, in which there were no toxic, irritant, noxious, and environmentally hazardous solvents compare to the available commercial inks [5].

Chen et al. investigated the rheological behavior of low temperature silver inks adaptable for screen printing through a thixotropy test, oscillatory stress sweep test, viscosity test and creep recovery test for crystalline silicon/amorphous (c-Si/a-Si:H) heterojunction solar-cell applications. They mentioned the necessity of an optimum shear thinning behavior to permit the ink to flow through the screen, and an appropriate thixotropic behavior to level the pattern 
without slumping. They found that the rheological behavior of those inks strongly depended on their composition [58].

Litchfield et al. reviewed various works on the rheological behavior of suspensions of nano-scale fillers with high aspect ratio. They found that for similar rheological behavior, much lower content of nanocomposite would be required compared to the microstructure, which could be due to the larger surface area and presence of a nanoparticle-polymer network. They showed artificial enhancement of particle contents due to effect of particle agglomeration. They also showed that the structural network reforming after shearing for particles with high aspect ratio strongly depends on the thermodynamic behavior of the systems [38].

Cassagnau investigated the dynamics and linear viscoelastic behavior of molten polymers and suspensions filled with nanoparticles of different aspect ratios. For suspensions of nanoparticles with high aspect ratio, they showed the presence of interparticle interaction or network structure, which would lead to different rheological behavior [40].

Haisheng et al. studied the rheological behavior of the rod/tube-like nanofluids of Titanate Nanotubes (TNT) in Ethylene Glycol (EG). They showed that the shear thinning behavior of the suspensions strongly depends on the TNT content. Due to the effect of temperature on the rheological behavior of nanofluids, they assessed the relative importance of convective and Brownian diffusion. They theoretical investigations proved that the rheological behavior of the nanofluids depends on aggregation and particle shape [59].

Chandler used an activation energy method to investigate the non-Newtonian viscosity behavior of solutions of starch in carboxymethyl cellulose. They investigated the degree of structure rearrangement during flow. They used an activation energy model for the dependence of the 
relative viscosity on the shear rate. They found positive activation entropy, with decreasing relative viscosity with increasing shear rate [41].

Zhou et al. investigated the reversible alignment of the AgNWs during shearing. They found a relatively high shear thinning behavior of the AgNWs suspensions especially at high concentration of AgNWs. They proved that at high shear rate, the arrangement of AgNWs was stable for a minimum time of $6000 \mathrm{~s}$. They observed that due to the special rheological behavior of $\mathrm{AgNW}$ and their rotation, the random arrangement could be recovered by removing the shear rate after at least $6000 \mathrm{~s}[60]$.

\subsection{Research Direction}

As it is clear from this literature review metal one-dimensional (1D) nanostructures can play a valuable role in electronic devices, and because of the high conductivity of bulk silver, it is one of the most promising nanowires. They also indicate the necessity to synthesis AgNWs in an efficient and inexpensive way. Even though AgNWs are commercially available, their synthesis is usually expensive and time consuming, and the final products are expensive.

The polyol synthesis of AgNWs is simple and provides a relatively high yield. As reported in previous studies, without precise control the end product will be a mixture of nanostructures other than nanowires. Moreover, due to poor reaction control in a batch synthesis of AgNWs, there is significant batch-to-batch variation. The batch reaction polyol process on large scales is time consuming and provides a low yield of AgNWs, irregular morphology of the wires and low aspect ratio. Finally, it is difficult to control the morphology of the silver nanowires based on reaction conditions. It is essential to optimize the polyol synthesis and determine the optimized reaction conditions to attain high yield of high aspect ratio AgNWs. There are some studies regarding the effect of process parameters on the polyol process for AgNWs. However, we are 
aware of no study that investigated the effect of the simultaneous variation of multiple parameters on the nanowire yield. A full investigation of the effects of interactions between all the essential parameters based on Full Factorial Design (FFD) requires a very large number of experiments. However, Design of Experiments (DoE) procedures can be used to obtain the largest feasible amount of information about the process through the smallest number of experiments.

Moreover, the application of millifluidic polyol synthesis can overcome the challenges in batch synthesis through control the reaction condition in small volume of reaction.

Previous studies show that for similar rheological behavior of conductive inks, much lower content of nanocomposite would be required compared to microstructures, which could be due to the larger surface area of nanoparticles. The rheological behavior of the silver nanowires during screen printing as an economical and environmentally friendly technology to create conductive patterns is not well known or investigated. It is therefore interesting to formulate water-based AgNWs inks adaptable for screen printing with desired shear thinning thixotropic rheological behavior. In particular, it would be valuable to control rheology with the silver nanowires rather than polymer and rheological agents. The objective is to attain high electrical conductivity of the printed patterns with sharp line definition as high as commercially available silver based inks with as low as possible content of AgNWs. Finally, the application of high aspect ratio AgNWs to conductive ink formulation can dramatically reduce the melting temperature, and consequently sintering temperature that allows their printing on flexible substrates.

All of these considerations guide this study in two main directions. The first objective is high yield synthesis of high aspect ratio AgNWs through an optimized polyol process in an 
inexpensive, large scale, continuous, and efficient manner. The second objective is formulation of water-based and environmentally friendly AgNWs conductive inks adaptable for economical screen printing to create conductive patterns with sharp line definition and high conductivity. 


\section{CHAPTER 3}

\section{EXPERIMENTAL APPARATUS AND PROCEDURES}

\subsection{Introduction}

AgNWs were synthesized through the batch polyol process. A small-scale batch reactor was used to investigate the effect of reaction parameters on the morphology of the synthesized silver nanostructures. A large-scale batch reactor was then constructed to produce sufficient quantities of AgNWs for further study. In addition, AgNWs were synthesized in a millifluidic reactor to investigate the application of various reaction methods on the morphology and yield of AgNWs. To obtain data on the growth mechanism and the morphology of the nanostructure at different stages of the batch reaction, small amounts of reaction solution were collected during the reaction. Scanning Electron Microscopy (SEM, AMRAY 3300 EF) was used to characterize the morphology of the nanowires and the morphology of the nanostructures collected.

Water-based inks were formulated with synthesized and commercial AgNWs, water, Isopropyl Alcohol (IPA), and rheological agents. A plate-plate rheometer AR 550 was used to probe the rheological behavior of the formulated inks.

\subsection{Synthesis of Silver Nanowires}

\subsubsection{Batch Polyol Process}

In the small-scale batch synthesis, $15 \mathrm{~mL}$ of Ethylene Glycol (EG anhydrous, Sigma-Aldrich, 324558 ) in a glass flask was heated at $150^{\circ} \mathrm{C}$ for $1 \mathrm{~h}$ under continuous magnetic stirring. Reagent solutions of $4 \mathrm{mM}$ Copper (II) Chloride $\left(\mathrm{CuCl}_{2}\right.$, Sigma-Aldrich, 751944, 99\%), $0.147 \mathrm{M}$ Poly(Vinyl Pyrrolidone) (PVP, Sigma-Aldrich, 856568, Avg. MW: 55000), and 0.094 M of 
Silver Nitrate $\left(\mathrm{AgNO}_{3}\right.$, Sigma-Aldrich, 10220, 99.8-100.5\%) were prepared. After preheating, $120 \mu \mathrm{L}$ of $\mathrm{CuCl}_{2}$ solution was added, and the solution was heated for fifteen minutes. Finally, 4.5 $\mathrm{mL}$ of PVP solution and $4.5 \mathrm{~mL}$ of $\mathrm{AgNO}_{3}$ solution were added into the flask.

In the large-scale batch synthesis, $100 \mathrm{~mL}$ of Ethylene Glycol (EG anhydrous, Sigma-Aldrich, 324558) in a glass flask was heated at $150^{\circ} \mathrm{C}$ for $1 \mathrm{~h}$ under continuous mechanical stirring (PTEF impeller with dimensions of $19 \mathrm{~mm}$ width, and $60 \mathrm{~mm}$ length). Reagent solutions of $4 \mathrm{mM}$ Copper (II) Chloride ( $\mathrm{CuCl}_{2}$, Sigma-Aldrich, 751944, 99\%), 0.147 M Poly(Vinyl Pyrrolidone) (PVP, Sigma-Aldrich, 856568, Avg. MW: 55000), and $0.094 \mathrm{M}$ of Silver Nitrate $\left(\mathrm{AgNO}_{3}\right.$, Sigma-Aldrich, 10220, 99.8-100.5\%) were prepared. After preheating, $800 \mu \mathrm{L}$ of $\mathrm{CuCl}_{2}$ solution was added, and the solution was heated for fifteen minutes. Finally, $30 \mathrm{~mL}$ of PVP solution and $30 \mathrm{~mL}$ of $\mathrm{AgNO}_{3}$ solution were injected into the flask.

The reaction flasks were purged with Argon gas during the preheating and reaction periods. The color of the reaction mix changed from yellow to red and then to opaque olive green within 30 min, changed to a clear peach color, and finally changed to opaque gray within 90 minutes. After nanowire formation, the reaction flask was cooled to room temperature. The solution was centrifuged at $3000 \mathrm{rpm}$ for $20 \mathrm{~min}$ once with addition of acetone (PHARNCO-AAPER, C14E15003) and then three times with addition of DI water. The product was then stored in DI water. A schematic of the small-scale batch reactor is shown in Figure 3. 1. 


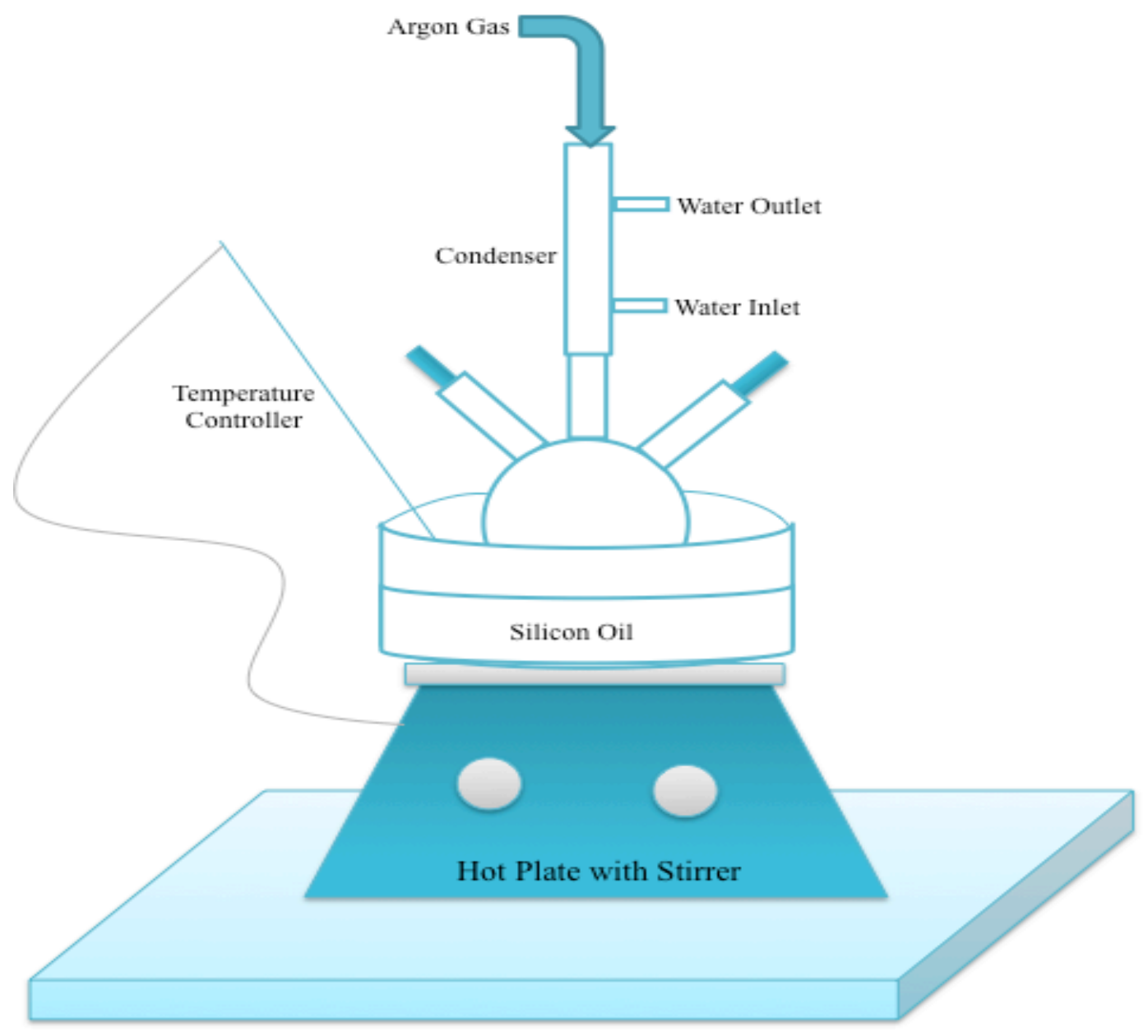

Figure 3. 1: Schematic of the small-scale batch reactor of polyol process.

\subsubsection{Millifluidic Polyol Process}

Silver nanowires were synthesized in a millifluidic reactor constructed from PTEF (Poly Tetra Fluoro Ethylene) tubing. Reagent solutions of $\mathrm{CuCl}_{2}, \mathrm{PVP}$, and $\mathrm{AgNO}_{3}$ with the same concentration in batch reactor were prepared. $60 \mu \mathrm{L}$ of $\mathrm{CuCl}_{2}$ solution was added to each PVP and $\mathrm{AgNO}_{3}$ solution. $4.5 \mathrm{~mL} \mathrm{AgNO}_{3}$ solution and $4.5 \mathrm{~mL}$ PVP solution were injected into the reactor with a syringe pump through a $135 \mathrm{~cm}$ length and $1.5 \mathrm{~mm}$ I.D at $40 \mu \mathrm{L} / \mathrm{min}$. PTFE tube that was placed in an oil bath controlled at a temperature of $150^{\circ} \mathrm{C}$. The PTFE tube discharged into a test tube in order to collect silver nanostructures. 
A schematic diagram of the millifluidic reactor is shown in Figure 3. 2. In order to see the effect of temperature on the morphology and yield of the synthesized AgNWs, the reaction was repeated at temperatures of $120^{\circ} \mathrm{C}, 130^{\circ} \mathrm{C}$, and $140^{\circ} \mathrm{C}$.

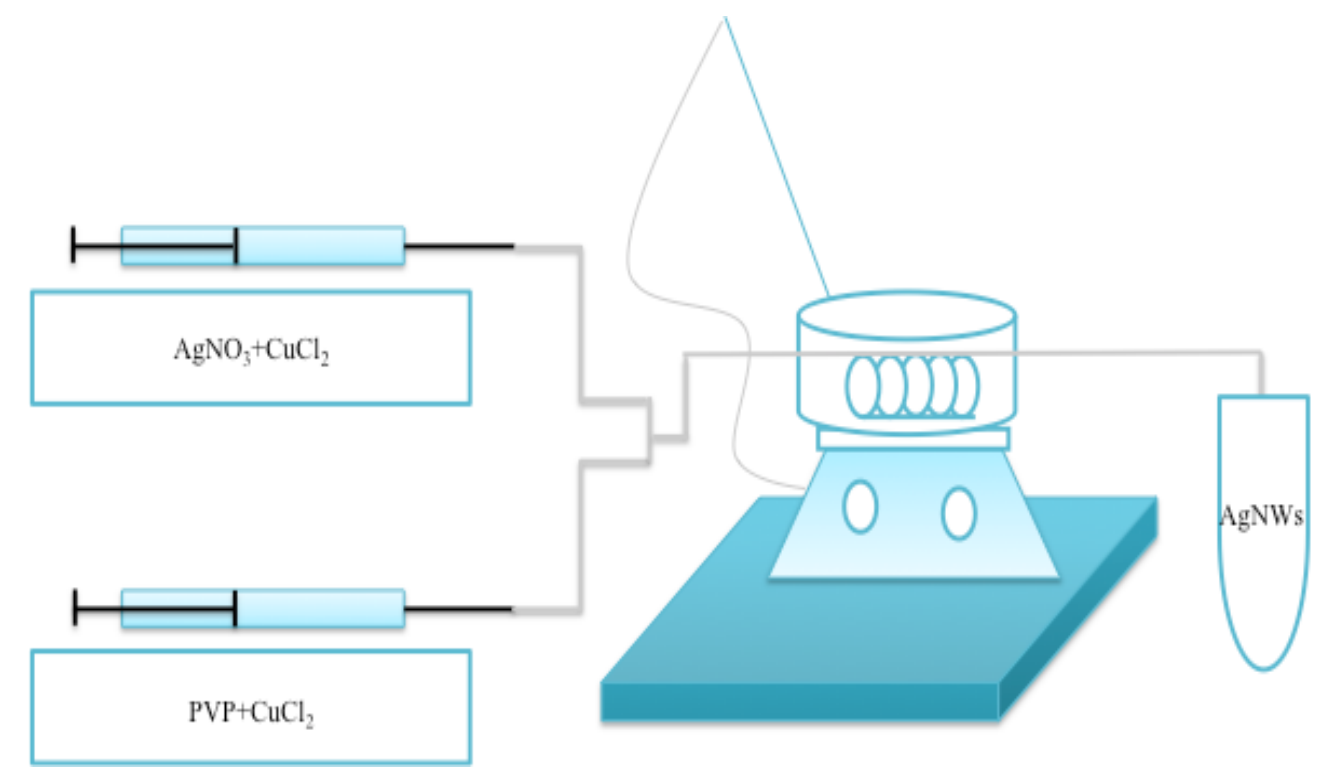

Figure 3. 2: Schematic of the millifluidic reactor of polyol process.

\subsection{Silver Nanowire Characterization}

Scanning Electron Microscopy (SEM, AMRAY 3300EF) was used to characterize the morphology of the synthesized silver nanowires and the morphology of the nanostructures collected during the reaction. For that purpose, a drop of each sample was placed on a piece of cover glass, dried and sputter-coated with Gold/Palladium (Au/Pd) to a thickness of $20 \mathrm{~nm}$. SEM images were acquired with a FEI field emission microscope operating at an accelerating voltage of $7 \mathrm{kV}$. The length and diameter of nanowires in the images were measured with the help of ImageJ software.

\subsection{Sensitivity Analysis of the Polyol Process}

In order to determine the essential parameters in the polyol process, one parameter was varied at a time. The parameters were temperature, $\mathrm{AgNO}_{3}$ concentration, PVP concentration and 
molecular weight, $\mathrm{CuCl}_{2}$ concentration, sonication time for $\mathrm{AgNO}_{3}$ solution preparation, and power of stirring. Reactions with $\mathrm{AgNO}_{3}$ concentrations of $0.047 \mathrm{M}, 0.094 \mathrm{M}$, and $0.188 \mathrm{M}$ were done to investigate the effect of $\mathrm{AgNO}_{3}$ concentration, PVP (Avg. MW: 55000) concentrations of $0.0735 \mathrm{M}, 0.147 \mathrm{M}, 0.249 \mathrm{M}$, and $0.588 \mathrm{M}$ to illustrate the effect of PVP concentration, and $\mathrm{CuCl}_{2}$ concentrations of $0 \mathrm{mM}, 2 \mathrm{mM}, 4 \mathrm{mM}$, and $8 \mathrm{mM}$ to explore the effect of $\mathrm{CuCl}_{2}$ concentration on the morphology of the silver nanostructures and yield of the silver nanowires. In order to examine the effect of sonication time of the $\mathrm{AgNO}_{3}$ solution, the solution was sonicated for $0,3,6$, and 9 min and the synthesis was repeated with each solution. To examine the effect of PVP molecular weight on the morphology of the nanostructures and yield of the nanowires the process was repeated for PVP with average MW of 1300000 at concentrations of $0.0735 \mathrm{M}$ and $0.147 \mathrm{M}$. To explore the effect of temperature, the silver nanostructures were synthesized at $140^{\circ} \mathrm{C}, 150^{\circ} \mathrm{C}, 160^{\circ} \mathrm{C}$ and $170^{\circ} \mathrm{C}$. The effect of the type of salt was examined through utilizing $\mathrm{NaCl}(4 \mathrm{mM})$, and both $\mathrm{NaCl}$ and $\mathrm{CuCl}_{2}(2 \mathrm{mM}$ each) as salt mediate. The reaction was done at stirring speeds of $\sim 400 \mathrm{rpm}, \sim 500 \mathrm{rpm}$, and a combination of both to explore the effect of stirring on the nanostructures morphology and nanowire yield.

\subsection{Design of Experiments (DoE)}

After the parametric study, it was found that the most important parameters in the polyol process having a dominant effect on the yield of silver nanowires are temperature and reagent concentrations. Therefore these parameters, which predominantly control the yield of AgNWs (the percent of the number of AgNWs divided by all Ag nanostructures) as response, have been considered for further analysis by DoE to examine not only the simultaneous effect of those parameter, but to optimize the process as well. A Response Surface Design (RSD) of the JMP pro 11 coupled with Box-Behnken Design (BBD) was utilized to see the simultaneous effect of 
the main parameters, and illustrate a response surface from the main parameters. The RSD is a set of advanced design-of-experiments methods used to understand and optimize the response by modeling a curved quadratic surface for continuous parameters of temperature and regent concentrations and pinpoint the maximize yield of AgNWs inside the parameter regions. BBD is an alternative for central composite design as the most popular RSD in which there are three levels per parameters. The response was yield of AgNWs, which has been defined as the percentage of AgNWs among all nanostructures produced. For this purpose, 27 syntheses (DoE1 to DoE-27) corresponding to several combinations of main parameters were done. The synthesized nanostructures were characterized by SEM, and Image-J software was used to calculate the yield of AgNWs compare to the other structures.

\subsection{Conductive Ink Formulation}

Water-based ink was formulated with the silver nanowires, DI water, and Isopropyl Alcohol (IPA, EM SCIENCE, PX1835T) as solvent or carrier, Carboxymethyl Cellulose (CMC, SigmaAldrich, 419237) as a binder or thickener and Dispex Ultra FA 4416 (Old Hydropalat 216), BASF) as a dispersive agent. Both thickener and dispersive agent were added in relatively low concentration $(<5 \%)$. Both synthesized silver nanowires and commercial silver nanowires (AgNWS-90 Blue Nano, and AgNWs-40 Blue Nano) were utilized as conductive filler to prepare conductive inks. The commercial AgNWs morphology (length and diameter) is similar to the morphology of the AgNWs synthesized in the millifluidic reactor, and it is expected that rheology of the ink formulated with commercial AgNWs will be similar to that of ink formulated with the product of the millifluidic reactor. 
In order to isolate the effect of the silver nanowire on the rheological behavior of the ink, ink with the same component concentrations was prepared with the commercial silver nanoparticles $(<100 \mathrm{~nm}$, Sigma- Aldrich, 576832) rather than nanowires.

\subsection{Rheological Tests and Experiments}

A plate-plate rheometer AR 550 was used to probe the rheological behavior of the formulated inks. The plate radius was $40 \mathrm{~mm}$ and the gap was $500 \mu \mathrm{m}$, giving a sample volume of 0.628 $\mathrm{cm}^{3}$. A pre-conditioning step at a shear rate of $0.11 / \mathrm{s}$ for $10 \mathrm{~s}$ was applied before each test to assure uniformity of the fluids.

A Stress Sweep (SS) test was done to determine the Linear Visco-Elastic Region (LVR) with a plot of $\mathrm{G}^{\prime}(\mathrm{Pa})$ (storage or elastic modulus which illustrates the capacity of the ink to store energy) and $\mathrm{G}^{\prime \prime}(\mathrm{Pa})$ (loss or viscous modulus that illustrates the fluidity of the ink) as functions of stress $(\mathrm{Pa})$. The SS test was also done to investigate further the change of ink behavior from elastic to viscous in response to stress increasing through the printing process. Moreover the SS test provided investigation of the ink structure modification during printing.

A Steady State Flow (SSF) test was done to probe the shear thinning behavior of the ink through plots of viscosity (Pa.s) versus shear rate (1/s). The SSF test was required to investigate how the viscosity changes in response to increasing shear rate during printing.

After the LVR determination, the ink was further characterized by a Frequency Sweep (FS) experiment at a stress less than the critical stress to determine the variation of $G^{\prime}$ and $G^{\prime \prime}$ as 
functions of angular frequency. The FS test provides more information about the effect of colloidal forces and the interactions between particles.

Finally, a Peak Hold (PH) test was done to mimic the screen printing process. In order to simulate the screen printing process, constant shear rates were applied in three intervals. The first step was shearing at $0.11 / \mathrm{s}$ for $30 \mathrm{~s}$ to resemble the charging of the ink onto the mesh before printing. The second was shearing at $2001 / \mathrm{s}$ for $30 \mathrm{~s}$ to represent the screen printing application. The last was shearing at $0.1 \mathrm{1} / \mathrm{s}$ for $2 \mathrm{~min}$ to simulate viscosity recovering after printing.

In order to investigate the effect of temperature on the build-up of the ink structure after printing, the $\mathrm{PH}$ test was repeated at temperatures of $288 \mathrm{~K}, 298 \mathrm{~K}, 308 \mathrm{~K}$, and $318 \mathrm{~K}$. In addition, the $\mathrm{PH}$ test was repeated at a lower recovery shear rate $(0.041 / \mathrm{s})$ to investigate the effect of shear rate on the build-up and viscosity recovery mechanisms. A schematic of the rheometer configuration is shown in Figure 3. 3.

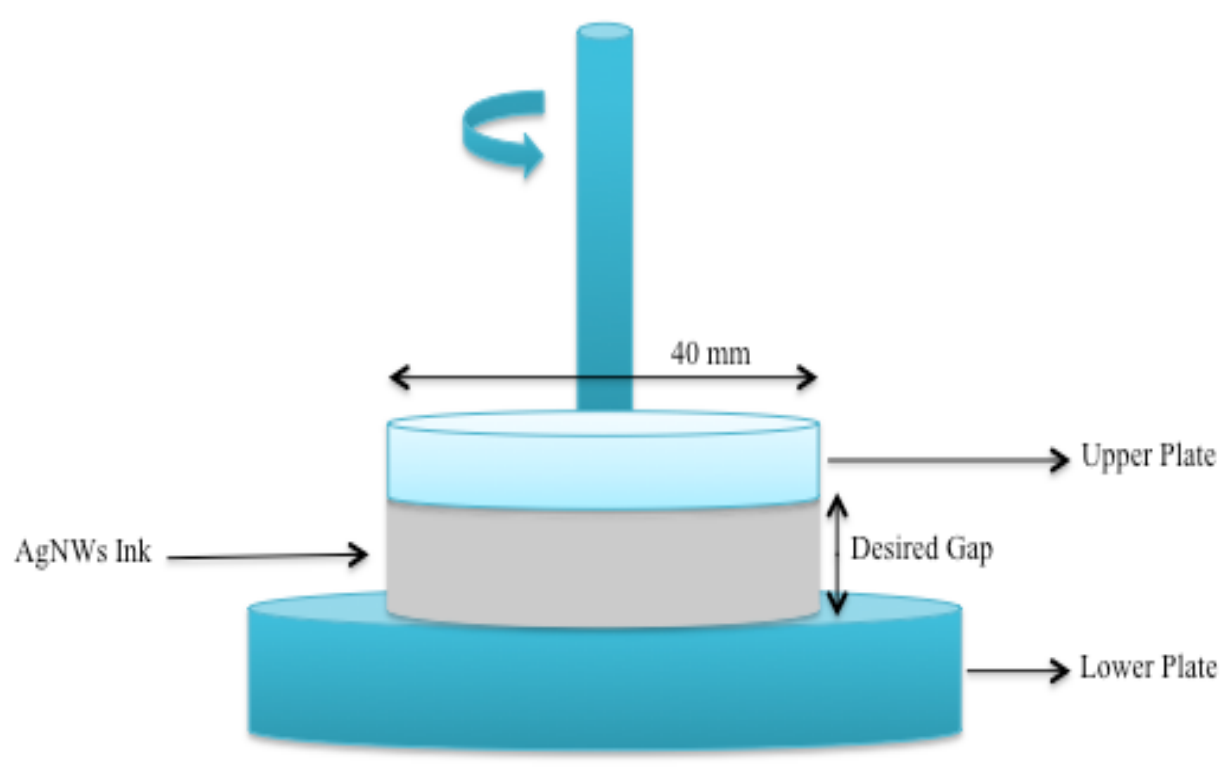

Figure 3. 3: Schematic of the rheometer configuration. 


\subsection{Screen Printing of Conductive Ink}

A small test circuit was printed onto a polycarbonate substrate with a manual screen printer. The manual screen printer was 325-mesh stainless steel screen with a wire diameter of 1.1 mil (milliinch). The screen mesh was set at a 22.5 degree angle to the frame, and the emulsion was 3 mils thick. A schematic of the screen printer is shown in Figure 3. 4.

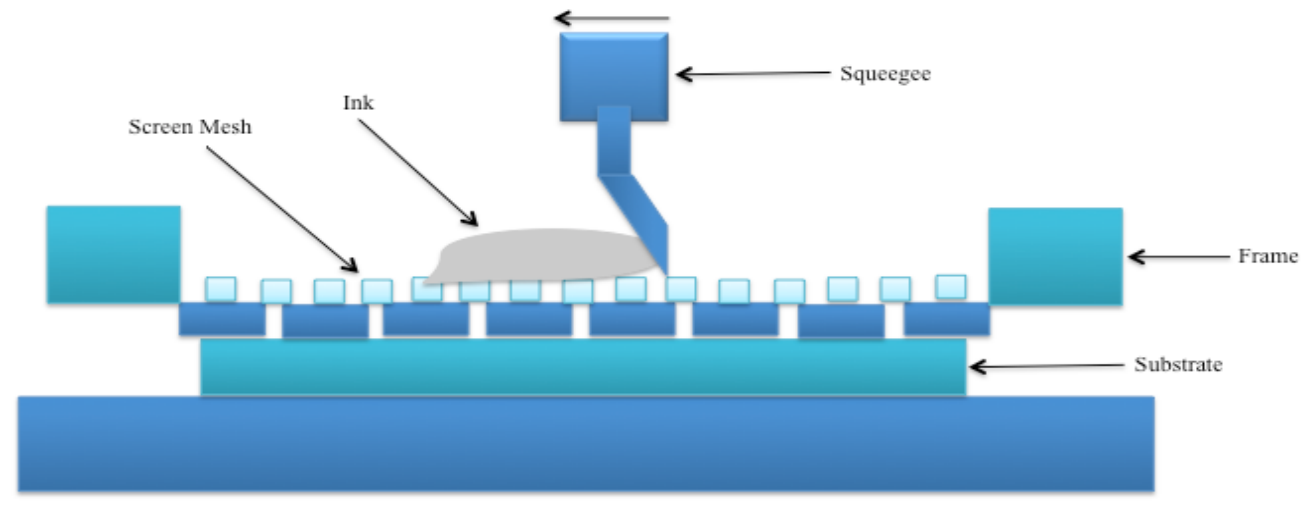

Figure 3. 4: Schematic of the screen printer.

\subsection{Characterization of Screen Printed Patterns}

Scanning Electron Microscopy (SEM, AMRAY 3300EF) was used to examine the microstructure of the printed ink after drying.

\subsection{Resistivity Measurements of the Screen Printed Patterns}

A Traceable Multimeter (FB61162) was used to measure the resistivity of the silver nanowire ink printed patters. The resistivity of the several content of AgNWs printed circuit was measured to investigate the effect of AgNWs content on the conductivity of the printed patterns. 


\section{CHAPTER 4}

\section{RESULTS AND DISCUSSION}

\subsection{Introduction}

Silver nanowires were synthesized in batch and millifluidic reactors by the polyol process. The silver nanostructures were characterized by SEM to investigate the morphology, diameter and length of the nanowires and to measure the nanowire yield and selectivity with respect to other structures. A sensitivity analysis was utilized to find the most important factors contributing to high yield and selectivity and to favorable wire morphology. A Design of Experiment (DoE) procedure was then followed to investigate the simultaneous effect of the essential factors on the AgNWs yield and to optimize the synthesis process.

Water-based inks were formulated with the synthesized AgNWs as well as with commercial AgNWs to act as both as conductive filler and rheological agents. Rheological tests including the Stress Sweep (SS) test, Steady State Flow (SSF) test, Frequency Sweep (FS) test, and Peak Hold (PH) test were done to investigate the rheological behavior of the formulated ink. The Stretched Exponential model (SEmo) was used to model the recovery process after screen printing to obtain the characteristic time of the recovery or build-up process. The build-up mechanism was characterized by a Peclet number. Activation energies of flow and of build-up were measured to investigate the recovery mechanism after screen printing as well. 


\subsection{Silver Nanowire Synthesis and Characterization}

\subsubsection{Batch Reactor}

The AgNWs were synthesized in batch reactors at scales from three times to twenty times compared to previously published work [1,2]. SEM (AMRAY 3300EF) was used to characterize the morphology of the synthesized silver nanowires. Figure 4. 1 shows SEM images of the products of the three, six, ten, and twenty times scaled-up batch processes in reactors of roughly $24,48,80$, and $160 \mathrm{~mL}$ volume, respectively. The reactor volume of the recipe in the previous study [1] is around $8 \mathrm{ml}$, which corresponds to scale-up factors for the reactors in this study of 3:1, 6:1, 10:1, and 20:1, respectively. The average diameters of the synthesized silver nanowires were $65,100,120$, and $88 \mathrm{~nm}$ for the three times, six times, ten times, and twenty times batches, respectively. The morphology of the synthesized silver nanostructures, and the dimensions of the synthesized silver nanowires suggested that thinner and longer AgNWs can be synthesized in three times scale-up batch reactor with magnetic stirring, and twenty times batch reactor with mechanical stirring. These results show that the reactor volume and stirring type strongly affect the morphology and dimension of the synthesized silver nanostructures. The standard deviation of the diameter was $21 \mathrm{~nm}$ for three times and $19 \mathrm{~nm}$ for twenty times. 

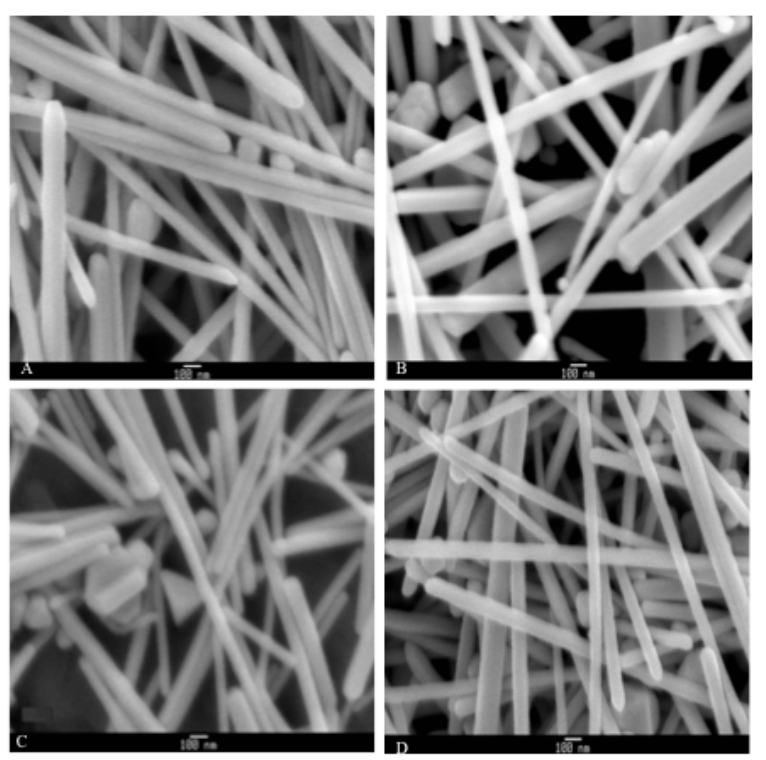

Figure 4. 1: SEM images of the synthesized silver nanowire from a batch reactor; A: three times scale-up, B: six times scale-up, C: ten times scale-up, and D: twenty times scale-up (scale bars: $100 \mathrm{~nm})$.

Figure 4. 2 shows SEM images of the synthesized AgNWs produced by the three times scale-up batch process at the same reaction conditions. It is clear that morphology of the silver nanostructures and the yield of silver nanowires vary for different batch reactors at the same volume. This is an example of the difficulties in control of the synthesis process, which is very sensitive to reaction conditions such as temperature and reagent concentrations. 

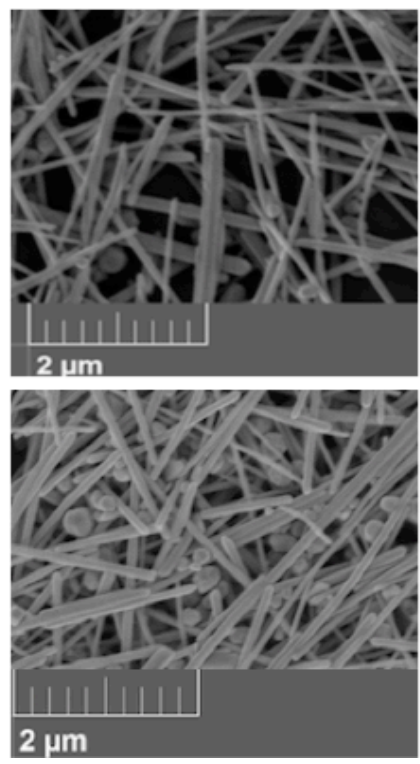
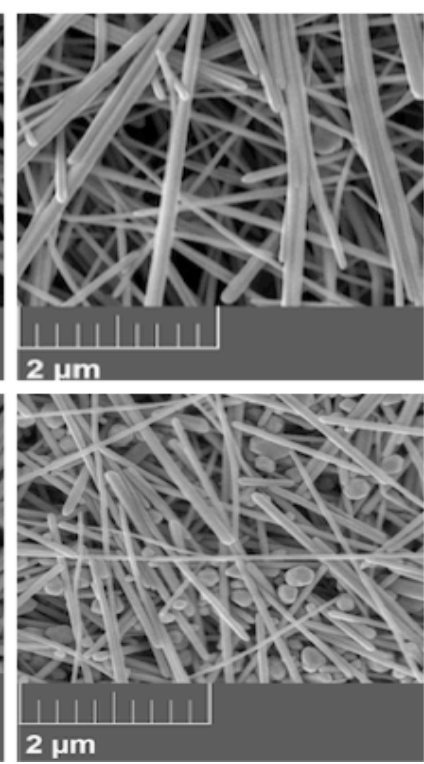
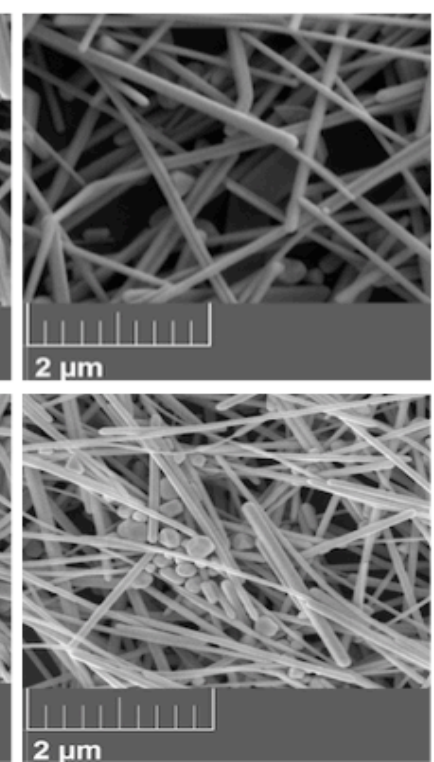

Figure 4. 2: SEM images of synthesized AgNWs in three times batch reactor through polyol process (scale bars: $2 \mu \mathrm{m}$ ).

\subsubsection{Millifluidic Reactor}

AgNWs were also synthesized in a millifluidic reactor. Figure 4. 3 shows SEM images of the synthesized AgNWs produced in the millifluidic reactor at the same conditions of reagent concentrations and temperature as in the batch process. It is clear that there are only nanowire structures, without any other structures produced as by products. Figure 4. 4 shows SEM images of the synthesized Ag nanostructures produced in the continuous millifluidic reactor at several temperatures. These SEM images illustrate that a high yield of AgNWs can be obtained in a continuous millifluidic reactor at temperatures as low as $130^{\circ} \mathrm{C}$.
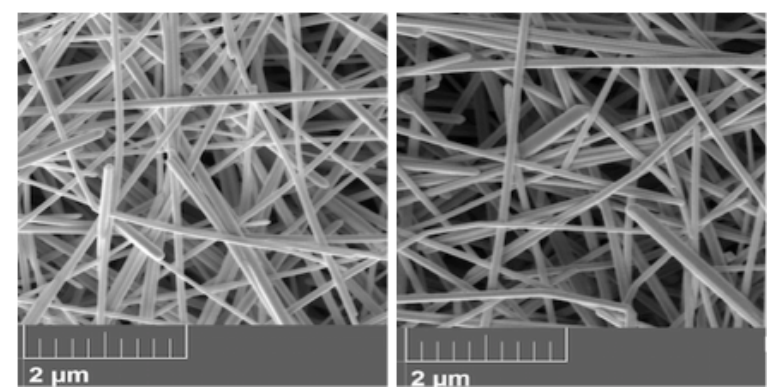

Figure 4. 3: SEM images of the synthesized silver nanowire produced in a millifluidic reactor (scale bars: $2 \mu \mathrm{m}$ ). 

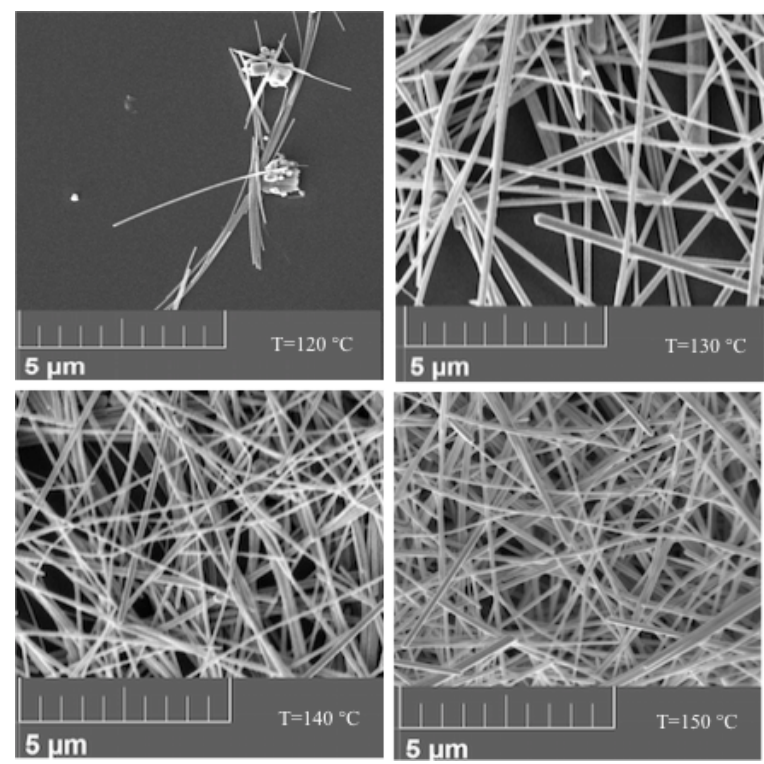

Figure 4. 4: SEM images of the synthesized AgNWs produced in a continuous millifluidic reactor through the polyol process at several temperatures (scale bars: $5 \mu \mathrm{m}$ ).

\subsection{Growth Mechanism}

SEM images of structures sampled during the first one and one half hours of the reaction in a batch process are shown in Figure 4. 5. Each nanostructure morphology corresponds to a specific color change during the reaction.
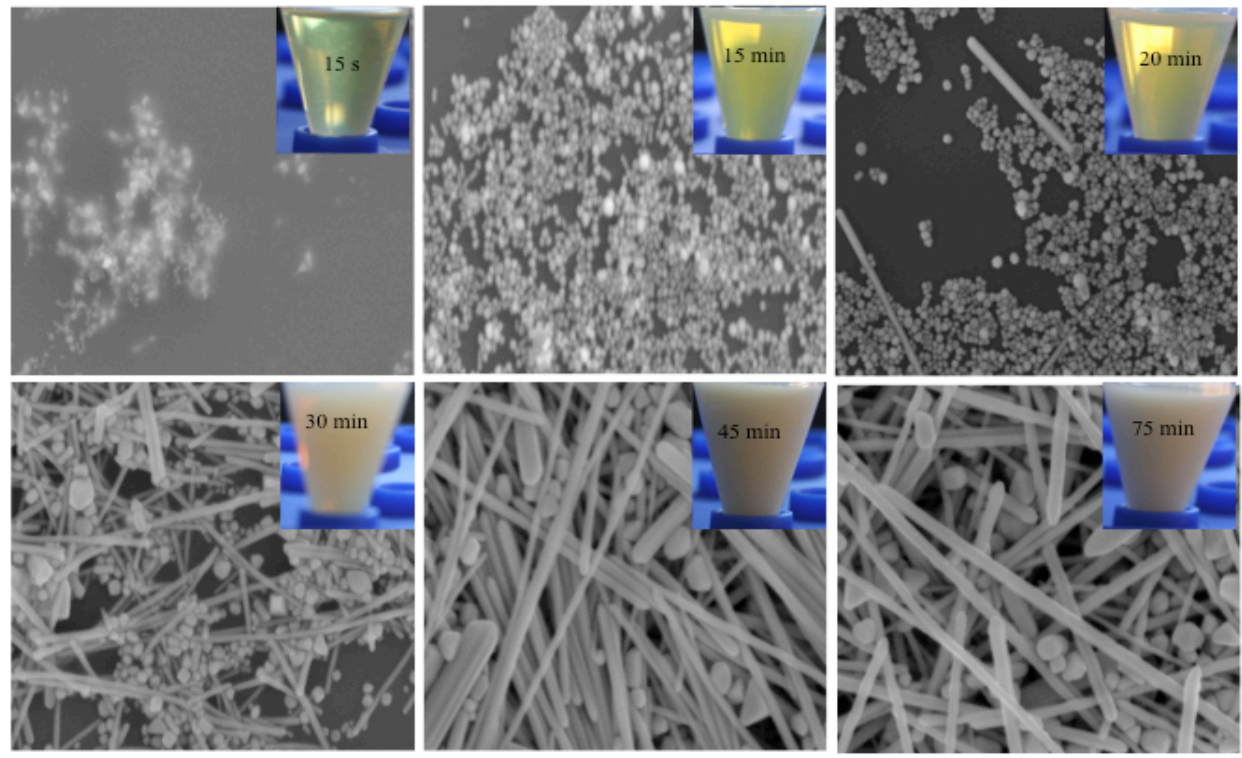

Figure 4. 5: SEM images of particles recovered at various stages of the synthesis. 
In the synthesis reaction, Ethylene Glycol (EG) is converted to Glycol Aldehyde (GA) at high temperature, and it reduces the $\mathrm{Ag}^{+}$ions to form small silver nanoparticles as shown in reaction $4.1[27]$

$$
2 \mathrm{HOCH}_{2} \mathrm{CH}_{2} \mathrm{OH}+\mathrm{O}_{2} \stackrel{150^{\circ} \mathrm{C}}{\longrightarrow} 2 \mathrm{HOCH}_{2} \mathrm{CHO}+2 \mathrm{H}_{2} \mathrm{O} \text {. }
$$

As is clear from the SEM images, the small silver nanoparticle seeds are not stable in solution, and they start to dissolve. At the same time, silver nanowires start to grow in the presence of PVP, which preferentially adsorbs on the $\{100\}$ facets of the silver nanocrystals. A schematic diagram of the formation of silver seeds and elongation of the seeds to the wire shape through the passivation of the $\{100\}$ facets is shown in Figure 4. 6 [7].

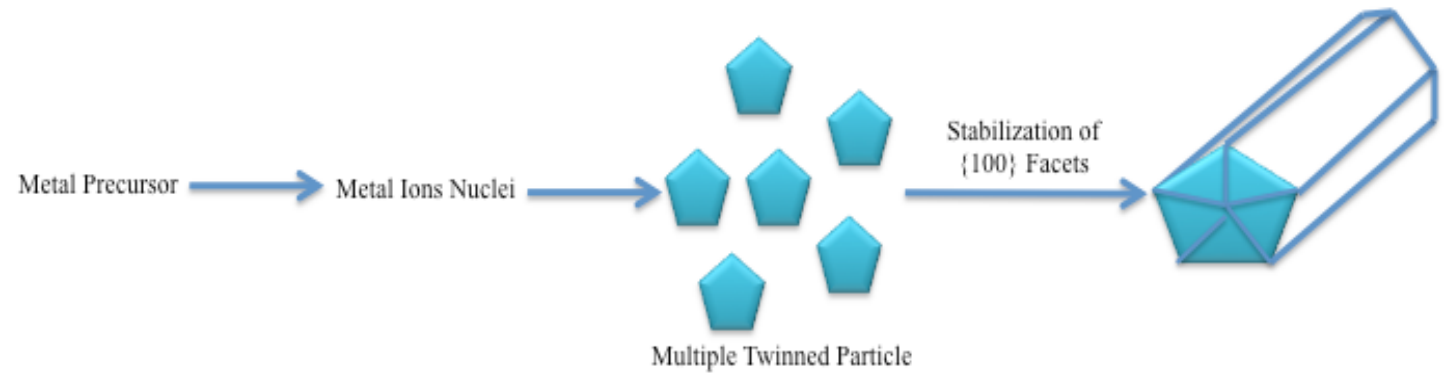

Figure 4. 6: Illustration of the growth mechanism [7].

A small amount of appropriate salt is essential in order to stabilize the seeds. The $\mathrm{Cl}^{-}$ions stabilize the silver seeds and reduce the concentration of free $\mathrm{Ag}^{+}$ions by formation of $\mathrm{AgCl}$ particles which act as nuclei as shown in reaction 4.2 [27]

$$
\mathrm{Ag}^{+}+\mathrm{Cl}^{-} \Leftrightarrow \mathrm{AgCl}
$$


The $\mathrm{Cu}^{+}$ions are formed by reduction of $\mathrm{Cu}^{2+}$ ions in the presence of $\mathrm{EG}$ as shown in reaction 4.3

$$
2 \mathrm{HOCH}_{2} \mathrm{CHO}+\mathrm{CuCl}_{2} \rightarrow 2 \mathrm{CH}_{2} \mathrm{COOH}+\mathrm{Cu}+2 \mathrm{HCl} .
$$

$\mathrm{Cu}^{+}$can scavenge oxygen atoms from the surface of the silver seeds and facilitate their growth into nanowires. The Argon purge has a similar function of removing oxygen from the surface of silver seeds. A schematic diagram of the oxygen adsorption from the surface of silver seeds is shown in Figure 4.7 [ 7].

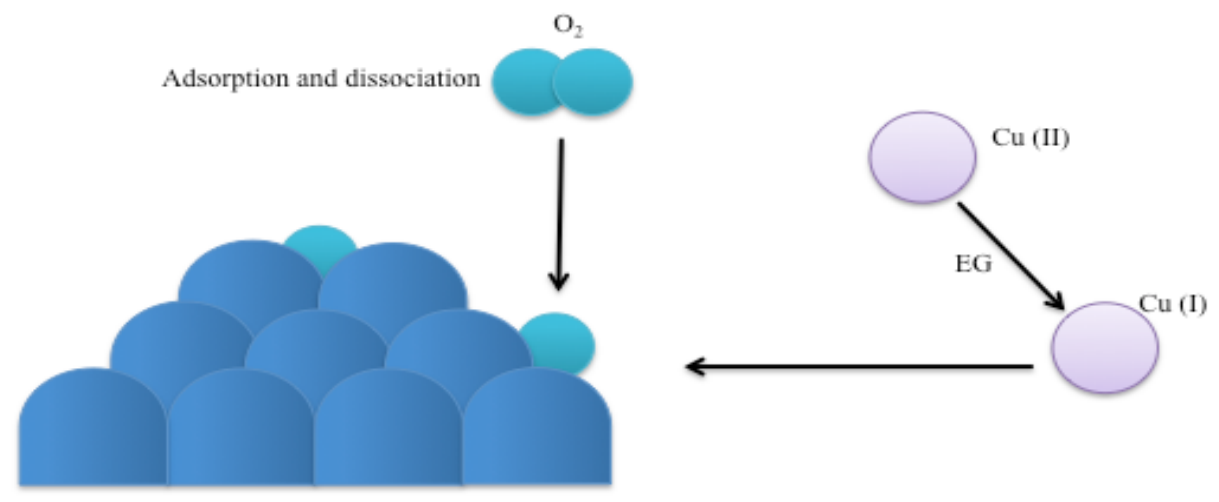

Figure 4. 7: Schematic depiction of oxygen adsorption from the surface of silver seeds [7]. In summary, there are three steps in the formation of silver nanowires through the polyol process including reduction, nucleation with the assistance of salt mediator, and wire growth governed by the capping agent PVP. This three-step proposed growth mechanism corresponds to SEM images obtained from the sample withdrawn during the reaction as shown in Figure 4. 8 . 


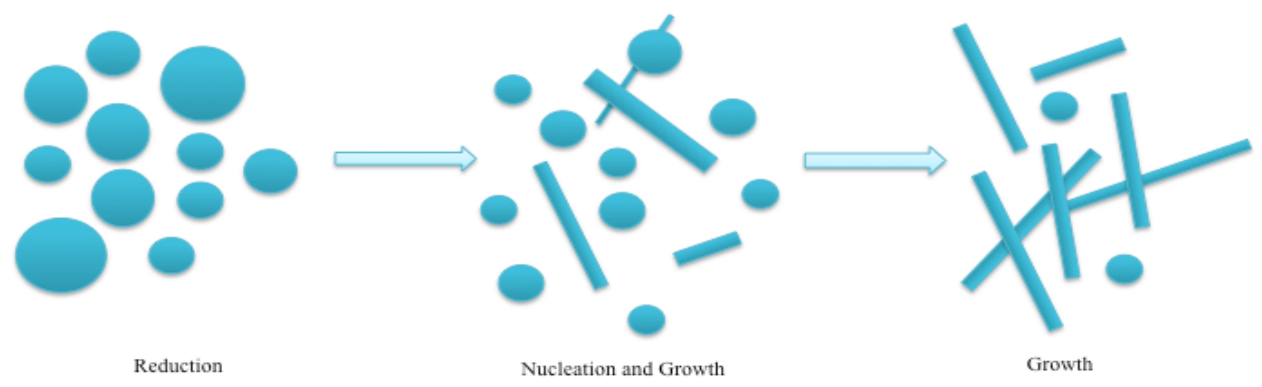

Figure 4. 8: Schematic of the silver nanowire growth mechanism of the polyol process.

\subsection{Sensitivity Analysis and Parametric Study}

The polyol process is complicated, sensitive and hard to control. Without accurate control of the reaction conditions, silver nanowires are produced in low yield. Moreover there are many byproducts such as unelongated nanoparticles, nanoplates, and nanocubes resulting from growth of isotropic seeds [47]. It is therefore essential to investigate the effects of different parameters such as PVP concentration and molecular weight, $\mathrm{CuCl}_{2}$ concentration, $\mathrm{AgNO}_{3}$ concentration, $\mathrm{AgNO}_{3}$ sonication time, reaction temperature, and power of mechanical stirring.

\subsubsection{Effect of Silver Nitrate Concentration}

In polyol synthesis of AgNWs, silver nitrate serves as metal salt precursor. In order to investigate the effect of the $\mathrm{AgNO}_{3}$ concentration on the morphology, yield, and dimensions of the synthesized AgNWs, reactions were performed with $\mathrm{AgNO}_{3}$ concentrations of $0.047 \mathrm{M}, 0.094 \mathrm{M}$, and $0.188 \mathrm{M}$.

Figure 4. 9 shows SEM images of $\mathrm{AgNWs}$ synthesized with $\mathrm{AgNO}_{3}$ concentrations of 0.047 , 0.094, and 0.188 M. As is clear from the images, the morphology and yield of AgNWs depends strongly on $\mathrm{AgNO}_{3}$ concentration. At low concentrations of $\mathrm{AgNO}_{3}$, the source of $\mathrm{AgNO}_{3}$ is insufficient for silver seed nucleation, and the synthesized nanostructures are mainly by-products such as particles, cubes, and triangle bipyramids. The yield of AgNWs increases with increasing $\mathrm{AgNO}_{3}$ concentration up to a limit. At very high concentration of silver nitrate, again there are 
some more other structure than 1D wires and rods structures because there are too many seeds competing for material $[46,48]$.
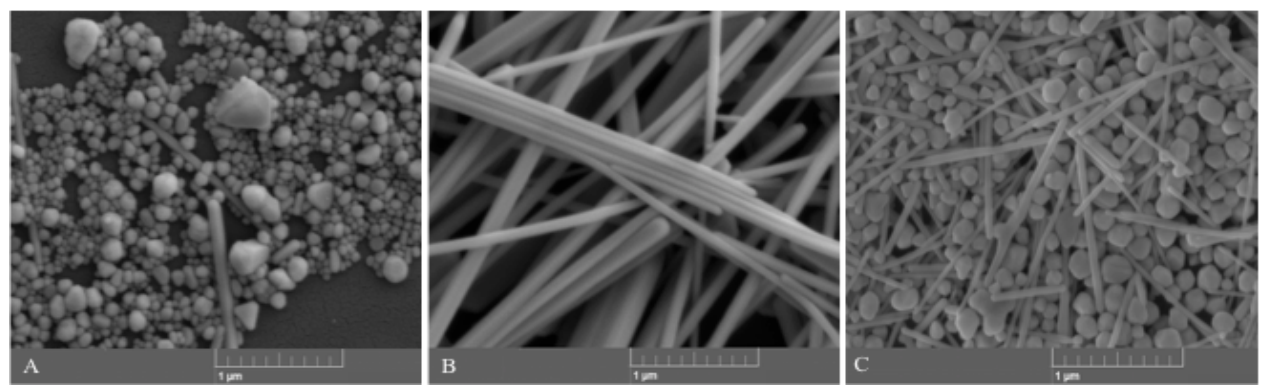

Figure 4. 9: $\mathrm{SEM}$ images of products at three concentrations of $\mathrm{AgNO}_{3} \mathrm{~A}: 0.047 \mathrm{M}, \mathrm{B}: 0.094 \mathrm{M}$, C: $0.188 \mathrm{M}$ (scale bars: $1 \mu \mathrm{m})$.

\subsubsection{Effect of Salt Type, and Copper Chloride Concentration}

Copper chloride was used as the source of chloride and copper ions in the process. To assess the effect of $\mathrm{CuCl}_{2}$ concentration, the process was repeated at $\mathrm{CuCl}_{2}$ concentrations of $0 \mathrm{mM}, 2 \mathrm{mM}$, $4 \mathrm{mM}$, and $8 \mathrm{mM}$. Moreover to explore the effect of salt type on the polyol process, the reaction was repeated with $4 \mathrm{mM} \mathrm{NaCl}$ as salt mediator, and with a combination of $2 \mathrm{mM} \mathrm{CuCl}_{2}$ and 2 $\mathrm{mM} \mathrm{NaCl}$. Figure 4. 10 shows SEM images of the silver nanostructures produced at several $\mathrm{CuCl}_{2}$ concentrations. Figure 4. 11 shows $\mathrm{SEM}$ images for comparison between $\mathrm{CuCl}_{2}, \mathrm{NaCl}$, and the combination of $\mathrm{CuCl}_{2}$ and $\mathrm{NaCl}$.

Without $\mathrm{CuCl}_{2}$ and at low concentration of $\mathrm{CuCl}_{2}$, only silver nanoparticles are produced. The best morphology with appropriate length and diameter distribution was obtained at $4 \mathrm{mM}$ concentration of $\mathrm{CuCl}_{2}$. 


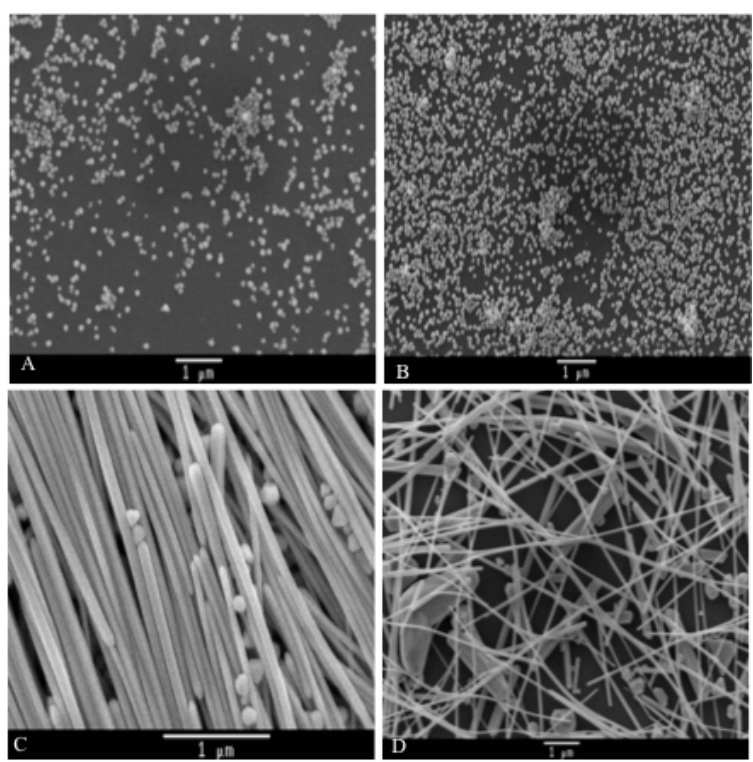

Figure 4. 10: SEM images demonstrating the effect of $\mathrm{CuCl}_{2}$ concentration; $\mathrm{A}: 0 \mathrm{mM}, \mathrm{B}: 2 \mathrm{mM}$, C: $4 \mathrm{mM}, \mathrm{D}: 8 \mathrm{mM}$ (scale bars: $1 \mu \mathrm{m}$ ).
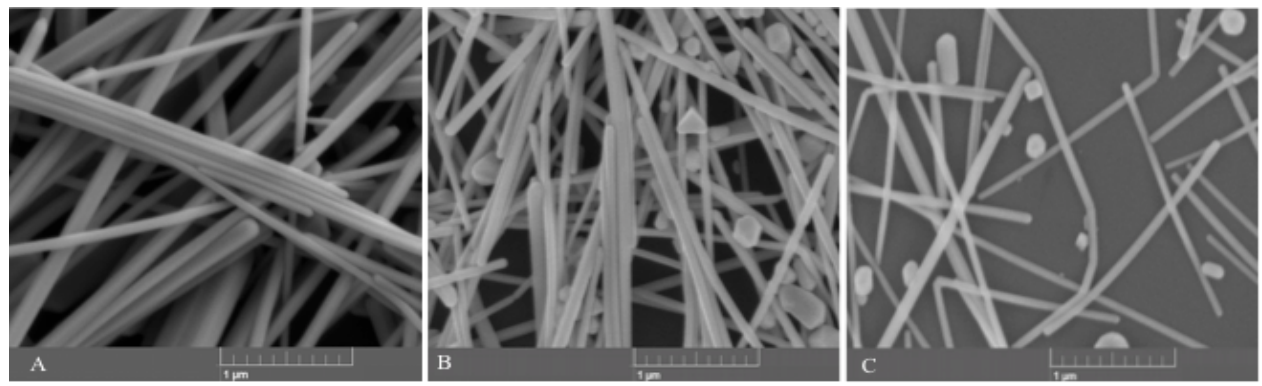

Figure 4. 11: SEM images demonstrating the effect of salt type; $\mathrm{A}: 4 \mathrm{mM} \mathrm{CuCl}$, B: $2 \mathrm{mM} \mathrm{CuCl}_{2}$ and $2 \mathrm{mM} \mathrm{NaCl}, \mathrm{C}: 4 \mathrm{mM} \mathrm{NaCl}$ (scale bars: $1 \mu \mathrm{m}$ ).

\subsubsection{Effect of PVP Concentration and Molecular Weight}

In the synthesis of AgNWs through the polyol process, PVP plays an essential role, acting as stabilizer, in controlling the growth and elongation of silver seeds to the wire shape. The concentration of PVP has a strong effect on the shape and size of synthesized AgNWs. PVPs with different molecular weights provide different degrees of stabilization for the silver seeds. Reactions with PVP (Avg. MW: 55000) concentrations of 0.0735M, 0.147M, 0.249M, and 0.588M were done to explore the effect of PVP concentration on morphology. To examine the effect of PVP molecular weight on the morphology of the nanostructures and yield of nanowires 
the process was repeated for PVP with average MW of 1300000 at two different concentrations of $0.0735 \mathrm{M}$, and $0.147 \mathrm{M}$. Figure 4. 12 shows the effect of PVP concentration, and Figure 4. 13 shows the effect of PVP molecular weight. PVP adsorbs on $\{100\}$ facets of the silver seeds. The coordination interaction strength between silver and PVP strongly depends on PVP molecular weight. A steric effect also influences the morphology of the resulting silver nanostructure. Short-chain PVP has a weaker steric effect, and it adsorbs on silver seeds more readily. However, coverage may be incomplete causing the silver nanostructures to aggregate. Longchain PVP inhibits aggregation, but the thick coating can decrease the coordination interaction strength. PVP with higher MW and longer chains provides more protection against aggregation, but weaker adsorption of PVP on the $\{100\}$ facets of silver seeds resulting in the growth of other nano structures such as twinned pentahedrons and nanoplates [47].

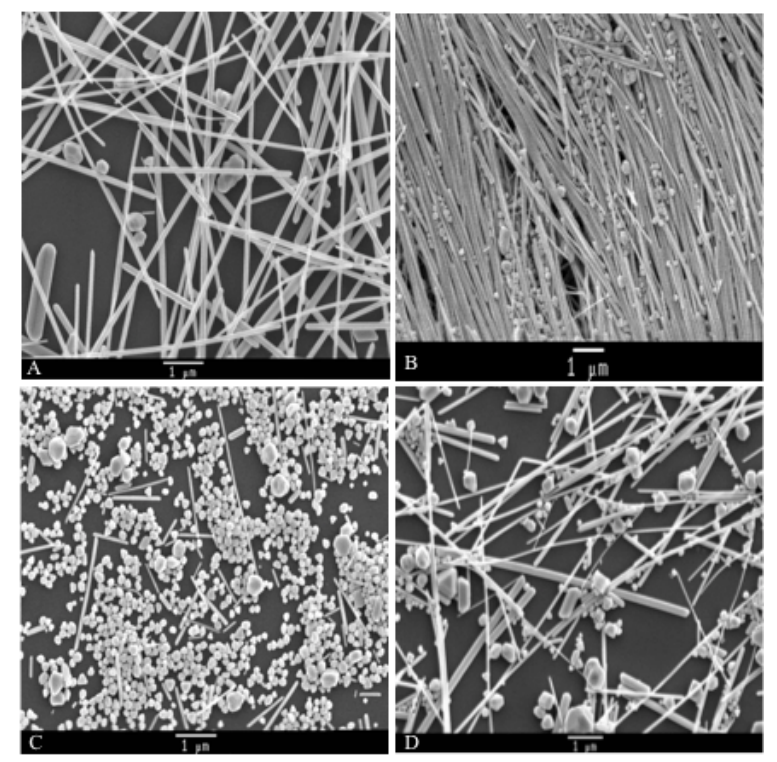

Figure 4. 12: SEM images demonstrating the effect of PVP concentration; A: 0.0735 M, B: 0.147 M, C: 0.294 M, D: 0.588 M (MW: 55000) (scale bars: $1 \mu \mathrm{m}$ ) 


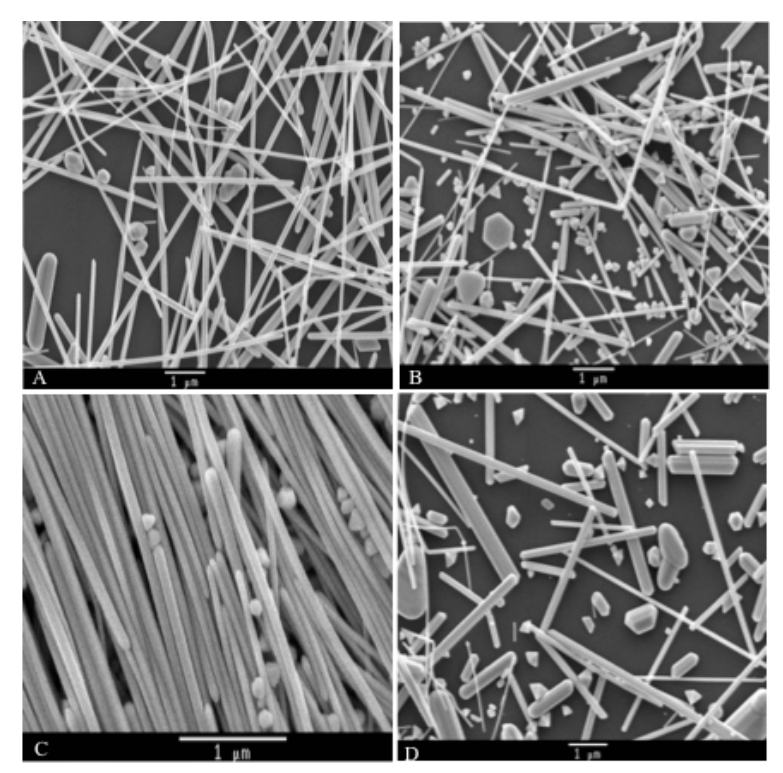

Figure 4. 13: SEM images demonstrating the effect of PVP MW; A: 0.0735 M (MW: 55000), B: 0.0735 M (MW: 1300000), C: 0.147 M (MW: 55000), and D: 0.147 M (MW: 1300000) (scale bars: $1 \mu \mathrm{m})$.

\subsubsection{Effect of Reaction Temperature}

The polyol process is very sensitive to temperature. To examine this sensitivity, the reaction was repeated at several temperatures from $140^{\circ} \mathrm{C}$ to $170^{\circ} \mathrm{C}$. In the initial stages of the reaction, high temperature is crucial for the conversion of ethylene glycol to glycol aldehyde. Then Ag atoms are nucleated through a homogeneous nucleation. Figure 4.14 illustrates the effect of the reaction temperature on the morphology and yield of the synthesized silver nanowires. It seems that at low temperature the conversion of the ethylene glycol to glycol aldehyde, and the reduction of silver ions and the color changes are very slow, which indicate formation of nuclei are accordingly slower. At high temperature the color change is rapid and there is not enough time for conversion of particles to wires. 

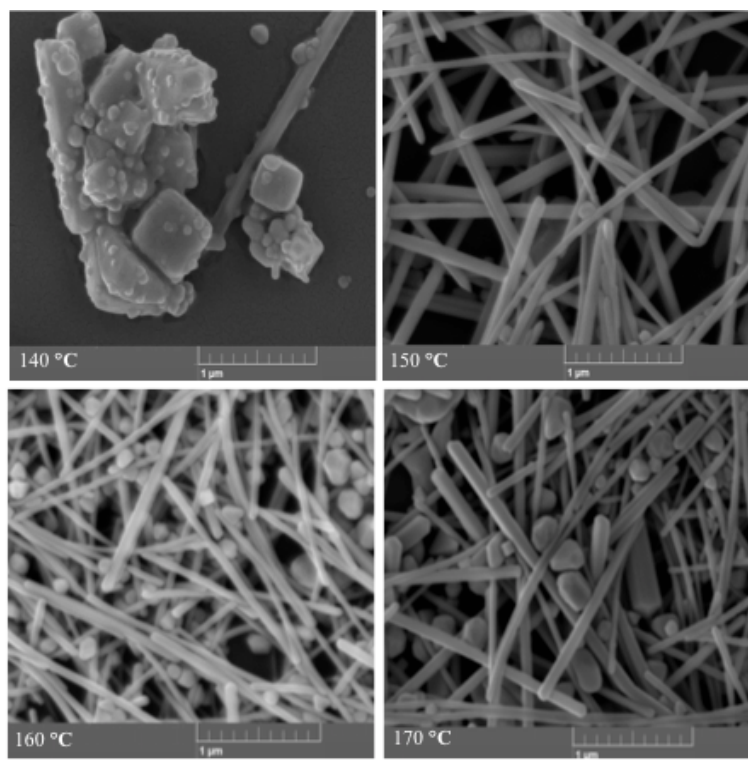

Figure 4. 14: SEM images demonstrating the effect of the reaction temperature (scale bars: 1 $\mu \mathrm{m})$.

\subsubsection{Effect of Sonication Time of the Silver Nitrate Solution}

$\mathrm{AgNO}_{3}$ was dissolved in $\mathrm{EG}$ for various durations prior to addition of other reagents. The color of the $\mathrm{AgNO}_{3}$ solution changed due to the formation of small particles as sonication time was increased. The color of the solution changed from colorless to transparent red as sonication progressed. Prolonged sonication turns the seed solution black, which signifies the formation of larger particles with sizes on the order of the wavelength of visible light. Figure 4.15 shows the synthesized $\mathrm{AgNW}$ from $\mathrm{AgNO}_{3}$ seed solutions that were sonicated for 0, 3, 6 and 9 min. It was found that 5-6 min sonication of the $\mathrm{AgNO}_{3}$ solution could minimize the byproduct particles formed in the subsequent reaction and thus produce very long AgNWs. Too much shorter or longer than the optimum sonication time would cause to the production of many short AgNWs with a large number of by-products and subsequently decrease the NWs synthesis yield. When sonication time was much lower than optimum, or the $\mathrm{AgNO}_{3}$ was dissolved by hand shaking, many short NWs and many by-product nanoparticles were produced. This is due to the presence of undissolved $\mathrm{AgNO}_{3}$ clumps, where free $\mathrm{Ag}^{+}$would easily deposit by Ostwald ripening 
causing in many particles production. When the sonication time was longer than the optimum one, the color of the solution changed to black. This could be influenced by the initial seed formation and growth to irregular and large seeds. However, well-sonicated $\mathrm{AgNO}_{3}$ precursor solutions resulted in reduced by-product [2].
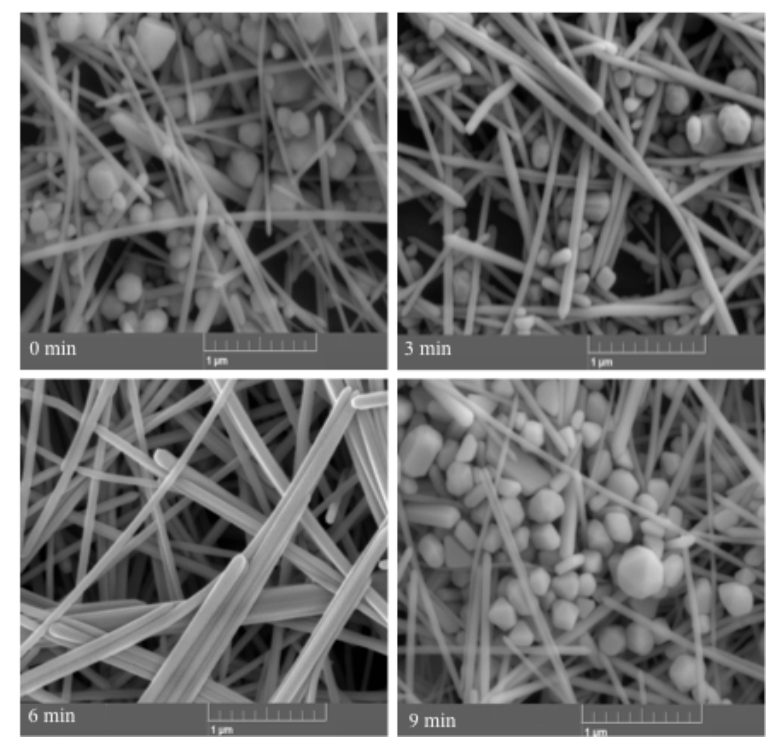

Figure 4. 15: SEM images demonstrating the effect of the sonication time of silver nitrate solution on the reaction products (scale bars: $1 \mu \mathrm{m}$ ).

\subsubsection{Effect of Mechanical Stirring Power}

Efficient mixing results in efficient generation of seeds and nanoparticles because high rates of heat and mass transfer are essential in the polyol process especially on the larger scale.

Low (360 rpm), high (480 rpm), and combination of the low and high (360 rpm during preheating, and $420 \mathrm{rpm}$ after regent injection) stirring was applied with a mechanical mixer. Figure 4. 16 illustrates the effect of stirring power on wire morphology. The speeds of $360 \mathrm{rpm}$, $420 \mathrm{rpm}$, and $480 \mathrm{rpm}$ correspond to the Reynolds number $\left(\operatorname{Re}=\mathrm{D}^{2} . \mathrm{N} . \rho / \mu\right.$ in which $\mathrm{D}$ is the impeller diameter, $\mathrm{N}$ is the speed in revolution $/ \mathrm{hr}, \rho$ is density, and $\mu$ is viscosity) of around 1495,1745 , and 1994, respectively. As is clear from the SEM images, a low stirring rate during 
preheating of the EG and high stirring after the injection of PVP and $\mathrm{AgNO}_{3}$ produced better morphology.
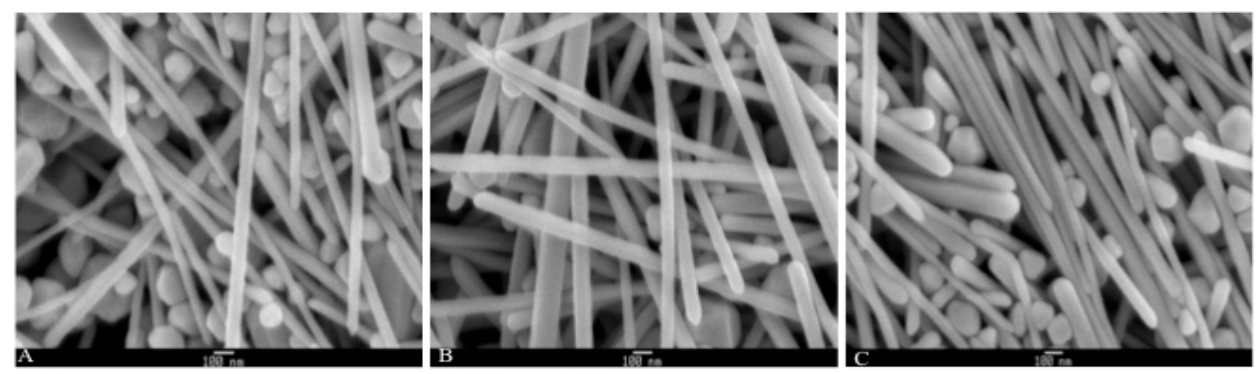

Figure 4. 16: SEM images demonstrating the effect of the power of stirring; A: low, B: combination of low and high, C: high (scale bars: $100 \mathrm{~nm}$ ).

\subsection{Design of Experiments (DoE)}

After the parametric study, it was found that the most important parameters affecting the yield of synthesized silver nanowires are temperature and reagent concentrations. These parameters, which predominantly control the yield of AgNWs as response, have been considered for a Design of Experiments (DoE) approach to examine not only the simultaneous effect of those parameters, but also to optimize the process. The Response Surface Design (RSD) of the JMP pro 11 coupled with Box-Behnken Design (BBD) has been utilized to evaluate the simultaneous effect of the main parameters, and illustrate a response surface from the main parameters. As shown in Table 4. 1, the yield of synthesized AgNWs was selected as the response. It is defined as the number percent of wires among the particles produced. Moreover, this table shows the essential parameters and corresponding two levels as -1 (corresponding to the initial value) and +1 (corresponding to the final value) of each parameter in the RSD. 
Table 4. 1: Response, factors, and actual amount of -1 and +1 levels.

\begin{tabular}{|c|c|c|c|}
\hline \multicolumn{4}{|c|}{ Response: Yield of AgNWs } \\
\hline Parameter & $\mathbf{- 1}$ & $\mathbf{0}$ & $\mathbf{1}$ \\
\hline $\mathrm{A}:$ Temperature $\left({ }^{\circ} \mathrm{C}\right)$ & 142 & 152 & 162 \\
\hline $\mathrm{B}: \mathrm{AgNO}_{3}$ Concentration $(\mathrm{M})$ & 0.05 & 0.09 & 0.14 \\
\hline $\mathrm{C}: \mathrm{PVP}$ Concentration $(\mathrm{M})$ & 0.07 & 0.15 & 0.22 \\
\hline $\mathrm{D}: \mathrm{CuCl}_{2}$ Concentration $(\mathrm{mM})$ & 2 & 4 & 6 \\
\hline
\end{tabular}

Figure 4. 17 shows SEM images of the nanostructures produced in the 27 experiments based on

DoE. It is clear that simultaneous changes of the four essential parameters can change the morphology of the synthesized silver nanostructures and yield of silver nanowires. For instance in DoE15, and DoE17 corresponding to $(-0+0)$, and (00+-) respectively, there are high yields of synthesized AgNPs. On the other hand, in DoE18 corresponding to $(++0+)$ there are high yields of synthesized AgNWs.

When the goal is process optimization, a high RSquare value is desirable, indicating the model is an appropriate response predictor. For this response, a RSquare value of $90 \%$ for AgNWs yield demonstrated that this expression is a good prediction for the response function of the four factors.

The analysis of this design based on $p$ value is shown in Table 4. 2. The p value assesses how each parameter is significant in the model. Based on a general rule of thumb, parameters are statistically important when the $\mathrm{p}$ value is 0.05 or lower. The fitted expression for the yield of synthesized AgNWs as function of four essential parameters is shown in equation 4.4 


$$
\begin{gathered}
\text { Yield }(\%)=83.33+17.55\left(\frac{\mathrm{A}-152}{10}\right)+7.97\left(\frac{\mathrm{B}-0.094}{0.047}\right)-3.61\left(\frac{\mathrm{C}-0.147}{0.0735}\right)+ \\
25.26\left(\frac{\mathrm{D}-4}{2}\right)+\left(\frac{\mathrm{A}-152}{10}\right)\left[1.91\left(\frac{\mathrm{B}-0.094}{0.047}\right)\right]+\left(\frac{\mathrm{A}-152}{10}\right)\left[-13.75\left(\frac{\mathrm{C}-0.147}{0.0735}\right)\right]+ \\
\left(\frac{\mathrm{B}-0.94}{0.047}\right)\left[-8.24\left(\frac{\mathrm{C}-0.147}{0.0735}\right)\right]+\left(\frac{\mathrm{A}-152}{10}\right)\left[9.47\left(\frac{\mathrm{D}-4}{2}\right)\right]+\left(\frac{\mathrm{C}-0.147}{0.0735}\right)\left[0.85\left(\frac{\mathrm{D}-4}{2}\right)\right]+ \\
\left(\frac{\mathrm{A}-152}{10}\right)\left[-23.93\left(\frac{\mathrm{A}-152}{10}\right)\right]+\left(\frac{\mathrm{B}-0.094}{0.047}\right)\left[-46.32\left(\frac{\mathrm{B}-0.094}{0.047}\right)\right]+ \\
\left(\frac{\mathrm{C}-0.147}{0.0735}\right)\left[-19.75\left(\frac{\mathrm{D}-0.147}{0.0735}\right)\right]+\left(\frac{\mathrm{D}-4}{2}\right)\left[1.91\left(\frac{\mathrm{D}-4}{2}\right)\right]
\end{gathered}
$$

The graphical representation of the model is shown in Figure 4. 18 through an interaction plot. In order to maximize desirability the yield of AgNWs, the prediction profiler is shown in Figure 4. 19 including corresponding values of temperature, $\mathrm{AgNO}_{3}$ concentration, PVP concentration, and $\mathrm{CuCl}_{2}$ concentration. The SEM image of the synthesized silver nanowire under optimized conditions as $\mathrm{T}=157.6^{\circ} \mathrm{C},\left[\mathrm{AgNO}_{3}\right]=0.102 \mathrm{M},[\mathrm{PVP}]=0.124 \mathrm{M}$, and $\left[\mathrm{CuCl}_{2}\right]=5.16 \mathrm{mM}$ is shown in Figure 4. 20. This image shows that under these conditions the yield of synthesized AgNWs is maximized. 

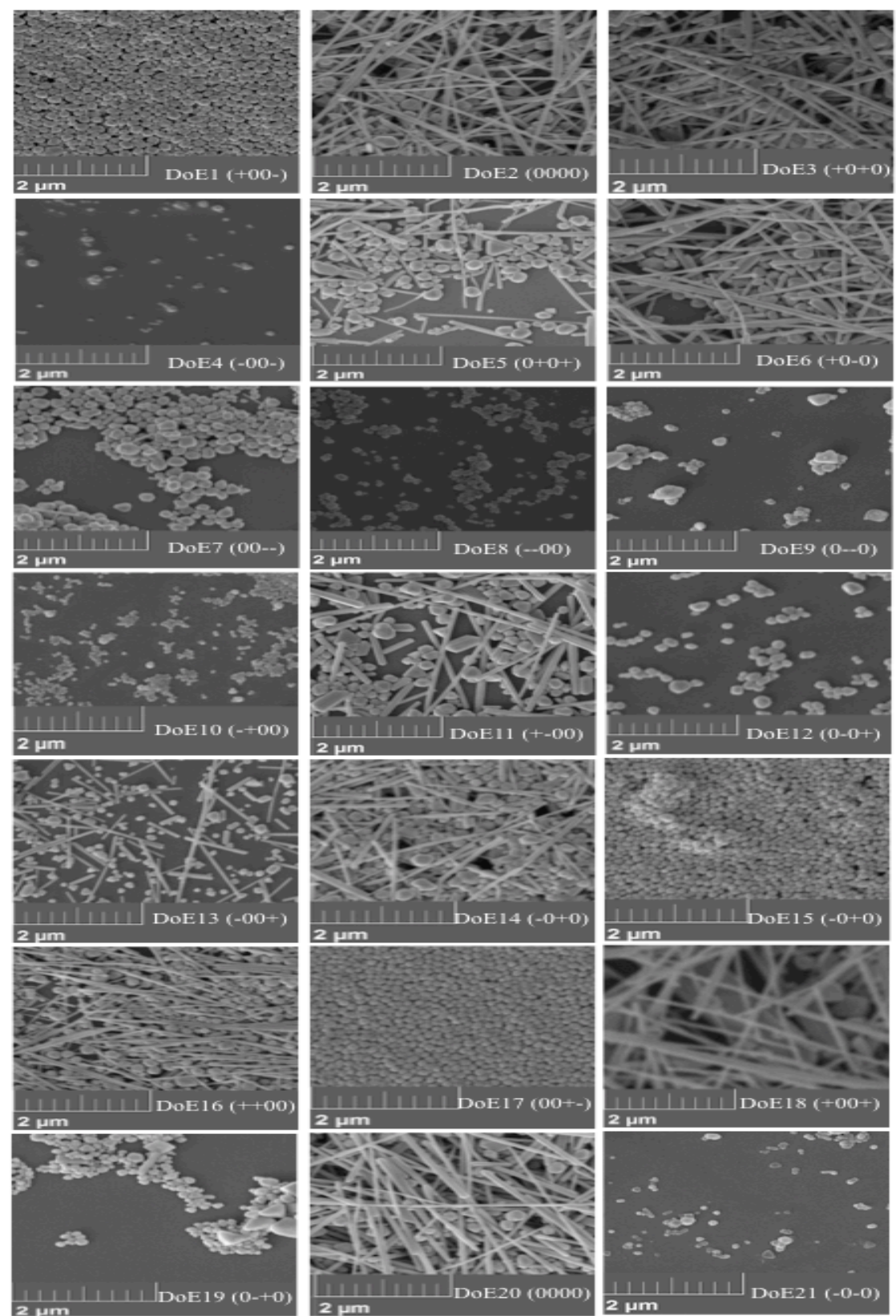

2 um
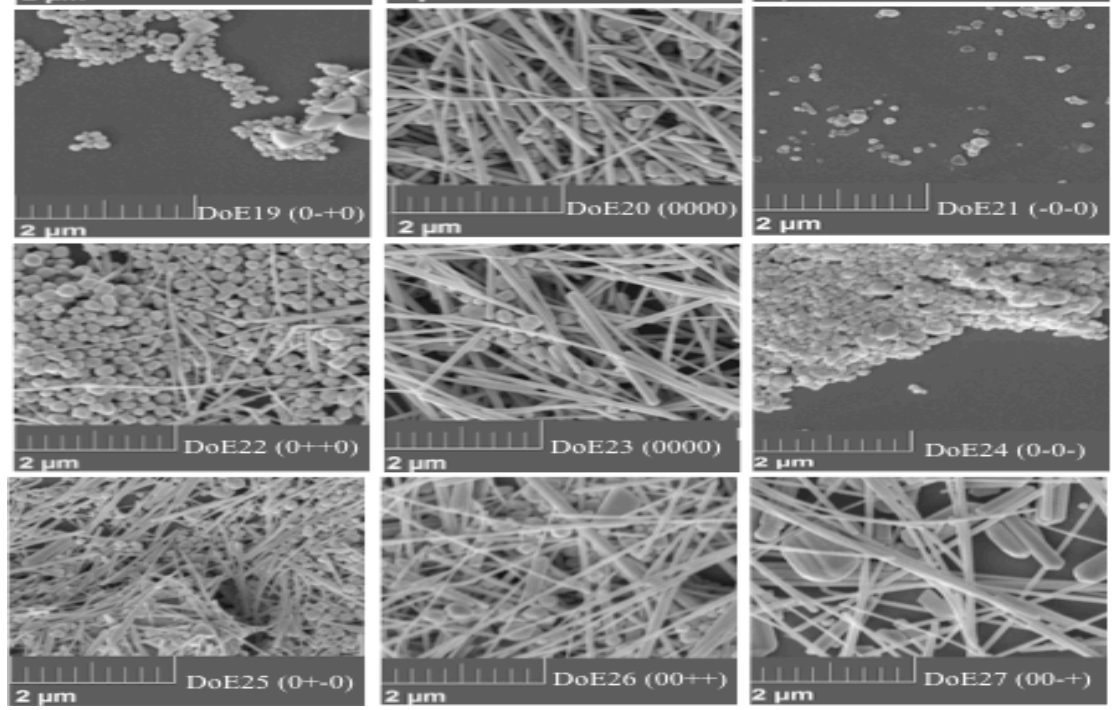

Figure 4. 17: SEM images of the 27 synthesized nanostructures based on the DoE (scale bars: 2 $\mu \mathrm{m})$. 
Table 4. 2: $\mathrm{P}$ value for yield of synthesized AgNWs surface response.

\begin{tabular}{|c|c|}
\hline Parameter & P value \\
\hline A & 0.0020 \\
\hline B & 0.1003 \\
\hline C & 0.4352 \\
\hline D & 0.0001 \\
\hline AA & 0.0039 \\
\hline BB & $<0.0001$ \\
\hline CC & 0.0123 \\
\hline DD & 0.0017 \\
\hline
\end{tabular}

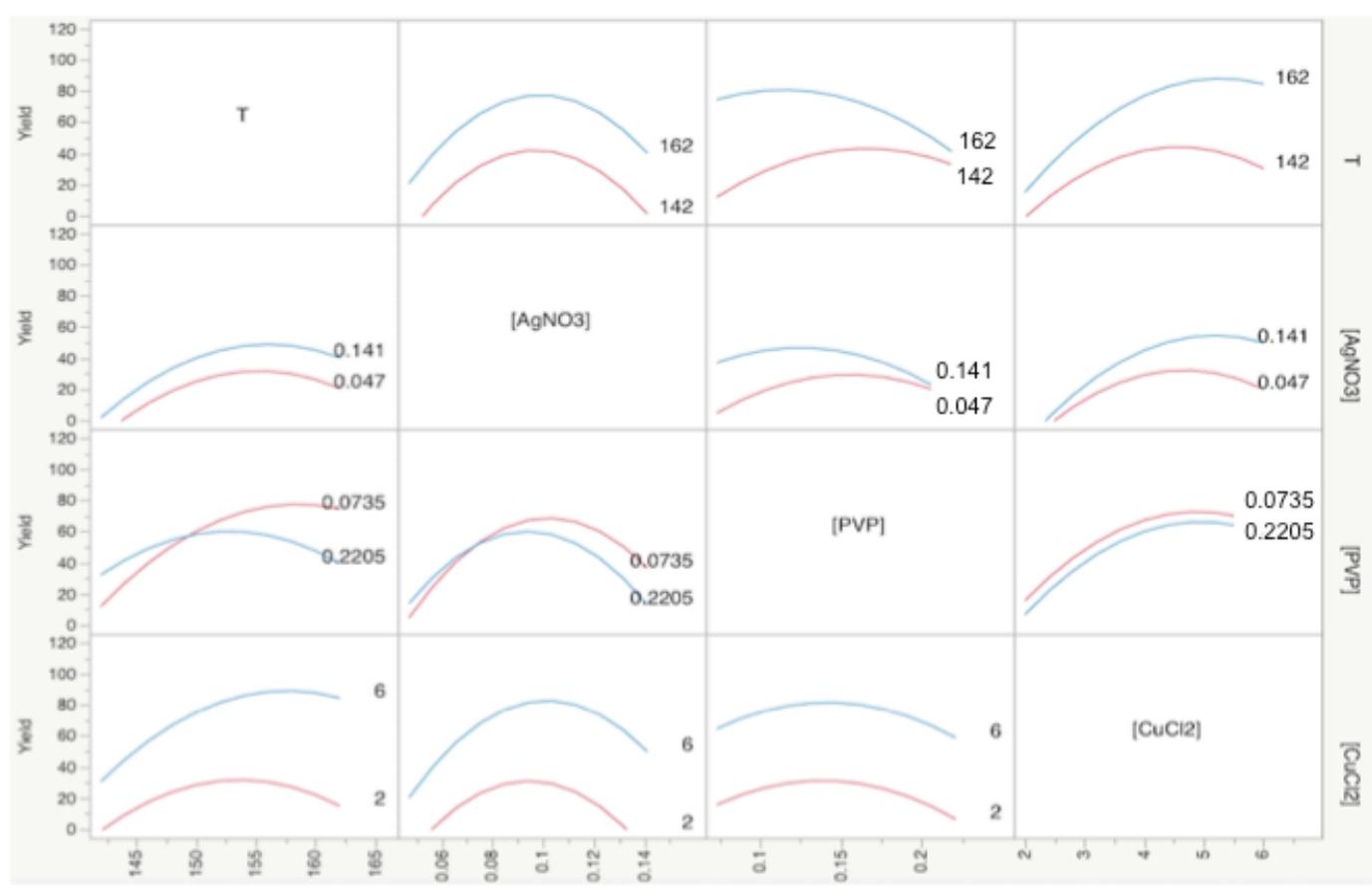

Figure 4. 18: Interaction plots for the yield of $\mathrm{AgNWs}$ for temperature, $\mathrm{AgNO}_{3}$ concentration, PVP concentration, and $\mathrm{CuCl}_{2}$ concentration.

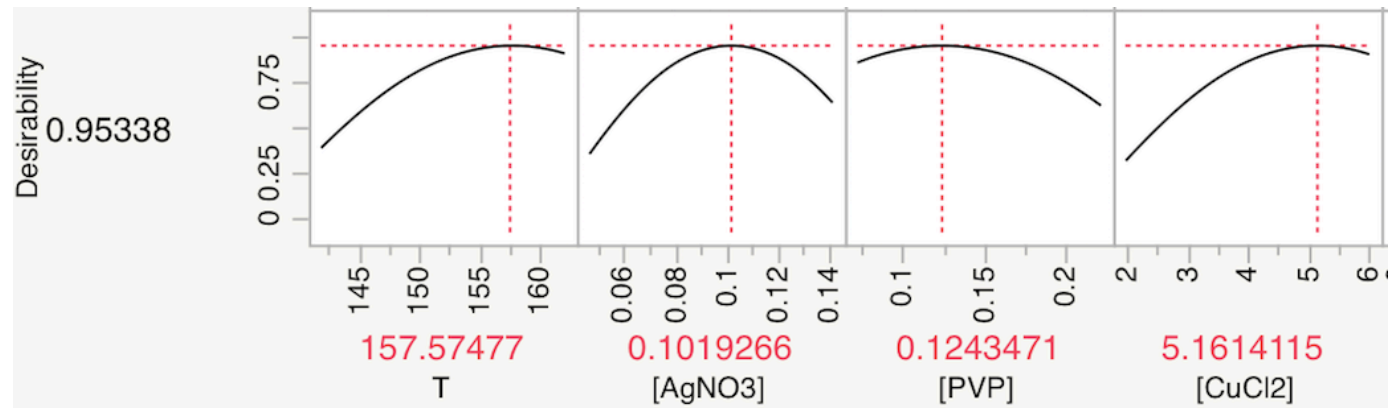

Figure 4. 19: Maximize desirability through prediction profiler including corresponding values for parameters. 


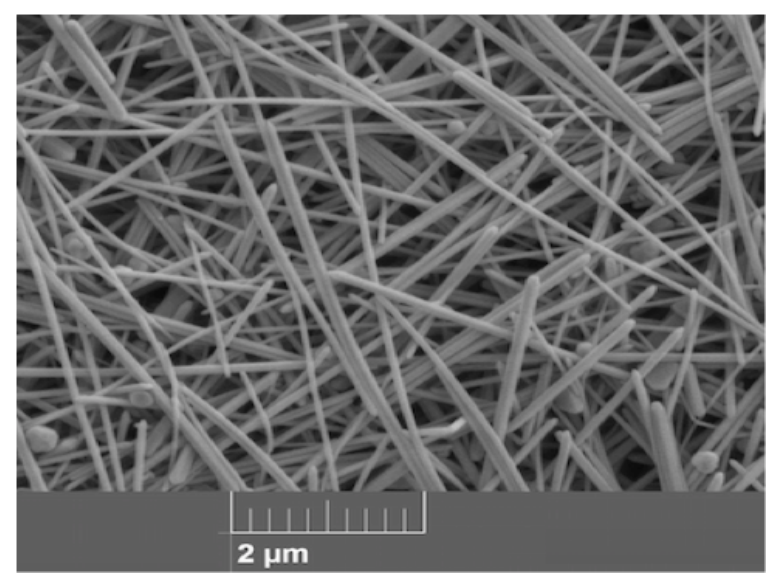

Figure 4. 20: SEM image of the synthesized AgNWs under the optimized conditions.

\subsection{Ink Formulation}

Water-based ink was formulated with both synthesized silver nanowires and commercial silver nanowires (AgNWs-90-Blue Nano, and AgNWs-40-Blue Nano), deionized (DI) water and Isopropyl Alcohol ((IPA), PHARMCO-AAPER, 231HPLC99) as solvent or carrier, Carboxymethyl Cellulose (CMC, Sigma- Aldrich, 419237) as a binder or thickener, and Dispex Ultra FA 4416 (Old Hydropalat 216), BASF) as a dispersive agent. In order to isolate the effect of the silver nanowire on the rheological behavior of the ink, ink with the same component concentrations was also prepared with commercial silver nanoparticles $(<100 \mathrm{~nm}$, SigmaAldrich, 576832) rather than nanowires.

\subsection{Rheological Tests and Results}

The investigation of the rheological behavior of suspensions can be challenging due to the necessity for an appropriate rheometer configuration, measurement protocol and data analysis suitable for suspensions. The parallel plate configuration was used rather than a cone and plate configuration because the finite size of the nanowires would complicate behavior near the apex of the cone. Because parallel plate configuration results in variation of shear rate with position, 
data analysis is based on comparison of results in experiments that differ only in ink composition.

\subsubsection{Stress Sweep Test (SS Test)}

A Stress Sweep (SS) test was done to determine the Linear Visco-elastic Region (LVR) with a plot of $\mathrm{G}^{\prime}(\mathrm{Pa})$ (elastic or storage modulus that measures the capacity of the ink to store energy) and $\mathrm{G}^{\prime \prime}(\mathrm{Pa})$ (viscous or loss modulus that measures the fluidity of the ink) as functions of stress (Pa). The SS test was done to investigate the change of ink behavior from more elastic to more viscous behavior in response to increasing stress during the printing process. Moreover this test permits investigation of changes in the ink microstructure during deformation and flow. Finally, it measures the LVR, in which ink structure is not changed by stress and shearing, and the $\mathrm{G}^{\prime}$ and $\mathrm{G}^{\prime \prime}$ are constant as function of stress. The result of the SS test for 50 wt.\% synthesized AgNWs ink, and 50 wt.\% commercial AgNPs ink is shown in Figure 4. 21. The result of the SS test for commercial silver ink (WB-101 provided by Conductive Compound Inc.) is shown in Figure 4. 22. 


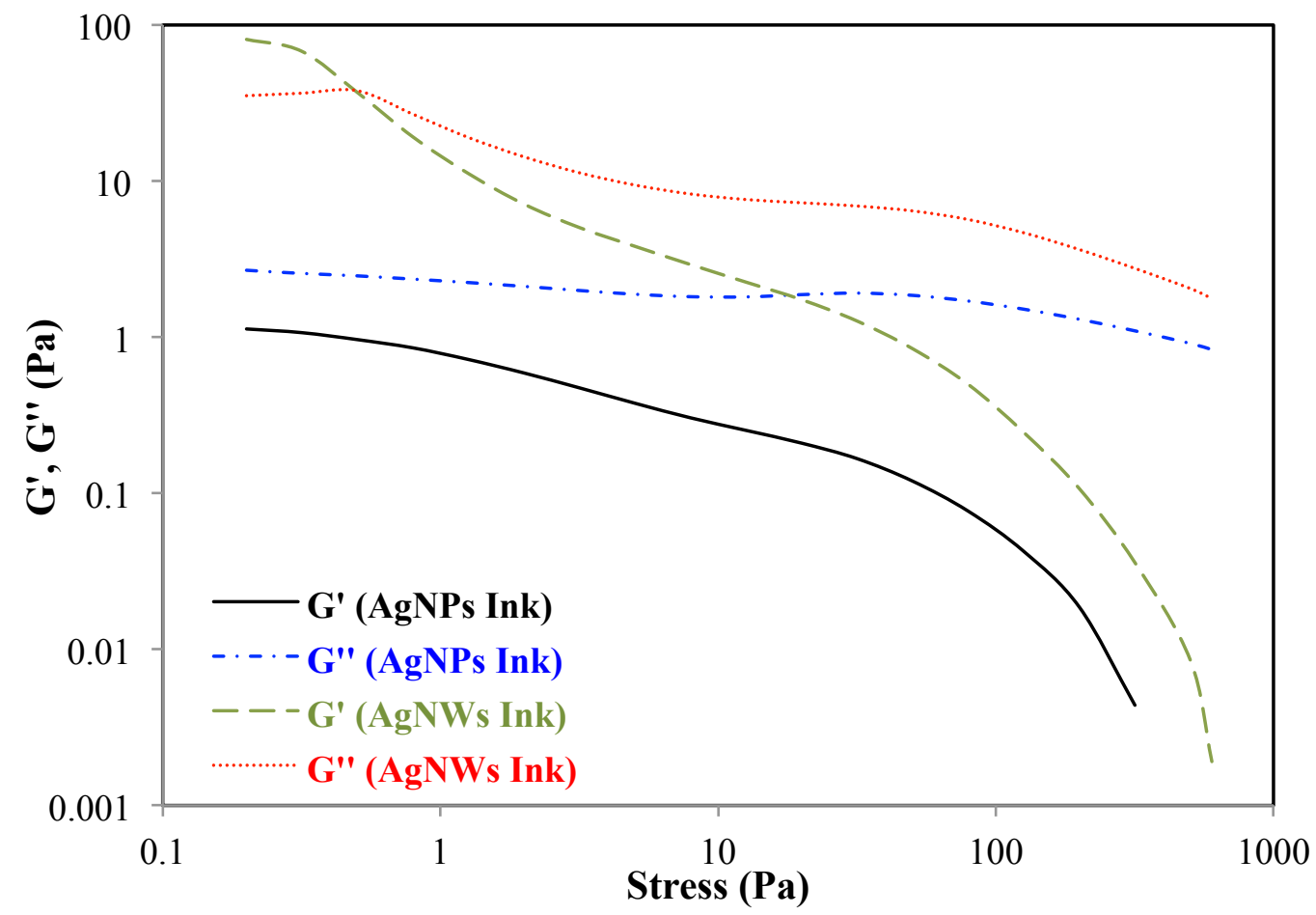

Figure 4. 21: G' and $\mathrm{G}^{\prime \prime}$ versus stress for nanoparticle and nanowire suspensions obtained in the stress sweep measurements.

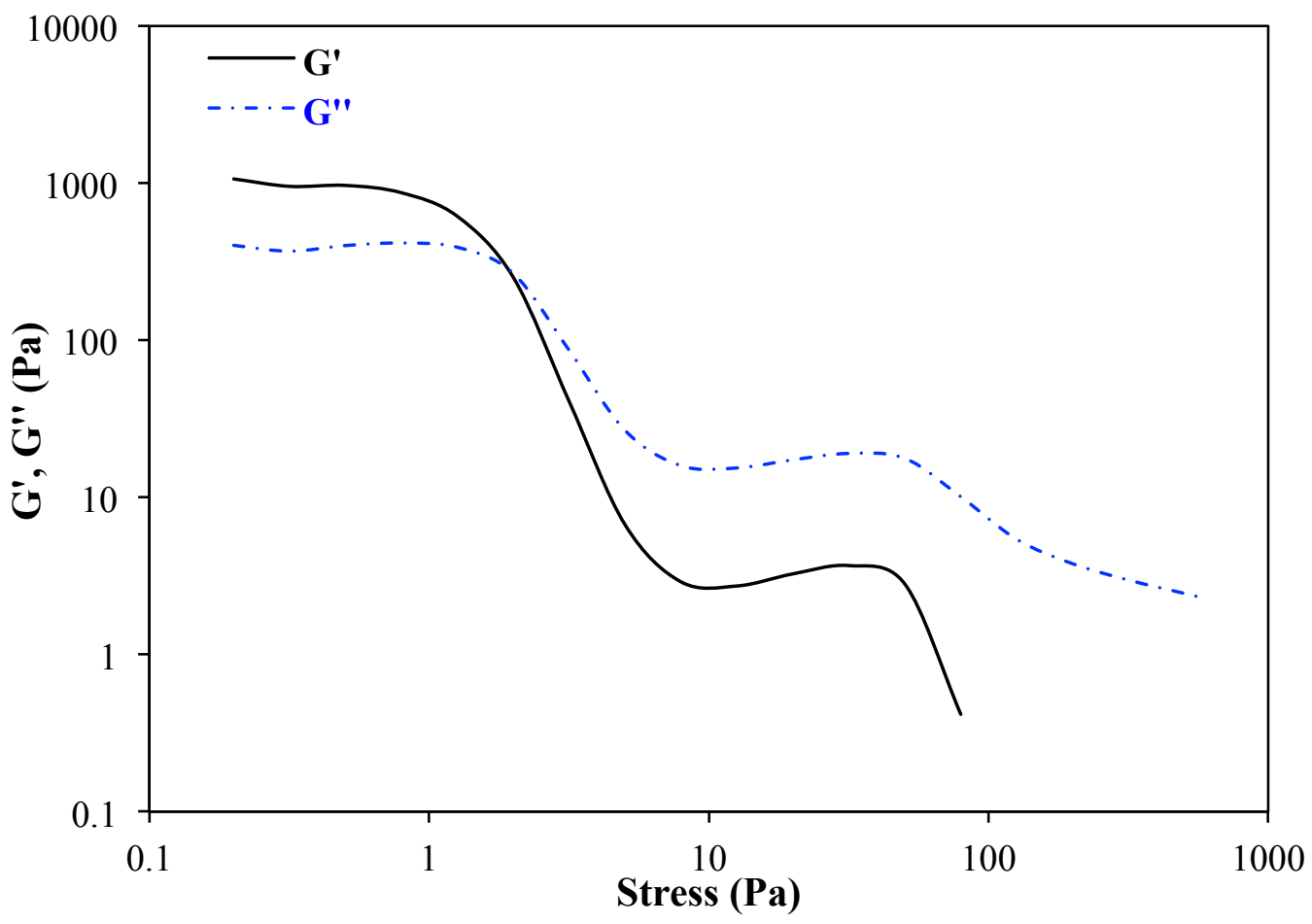

Figure 4. 22: G' and $\mathrm{G}^{\prime \prime}$ versus stress for WB-101 ink obtained in the stress sweep measurement. 
Based on the SS test results (see Figure 4. 21), the corresponding stress value of the Linear Visco-elastic Region (LVR) is $0.2 \mathrm{~Pa}$ for synthesized silver nanowire ink, and $0.5 \mathrm{~Pa}$ for commercial silver nanoparticle ink. This region of the diagram shows the range of shear stress at which the ink structure is not changed, and the values of $\mathrm{G}^{\prime}$ and $\mathrm{G}^{\prime \prime}$ are constant as function of stress. Above this range, the $G^{\prime}$ and $G^{\prime \prime}$ values decline continuously. There is a cross over point in the diagram for the synthesized silver nanowire ink, below which $G^{\prime}$ is greater than $G^{\prime \prime}$ corresponding to domination by the elastic behavior. Beyond this point at higher value of stress $\mathrm{G}^{\prime \prime}$ becomes larger than $\mathrm{G}^{\prime}$ corresponding to domination by the viscous behavior. For the commercial silver nanoparticle ink, $\mathrm{G}^{\prime \prime}$ is larger than $\mathrm{G}^{\prime}$ at all stresses in the SS experiments, and the behavior is dominated by viscosity. Based on the SS results for WB-101 ink (see Figure 4. 22), the corresponding stress value of the Linear Visco-elastic Region (LVR) is $1 \mathrm{~Pa}$.

\subsubsection{Steady State Flow Test (SSF Test)}

A Steady State Flow (SSF) test was done to probe the shear thinning behavior of the inks through plots of viscosity (Pa.s) versus shear rate $(1 / \mathrm{s})$. The SSF test is required to investigate how the viscosity changes in response to increasing shear rate during printing. Figure 4. 23 shows the results of the SSF of 50 wt.\% synthesized AgNWs, and 50 wt.\% commercial AgNPs. Figure 4. 24 shows the results of the SSF of commercial WB-101 ink. Although both inks show decreasing viscosity with increasing shear rate (see Figure 4. 23), the rate of the decline is higher for silver nanowire ink compared to the silver nanoparticle ink. The result of the SSF test for the WB-101 (see Figure 4. 24) ink also shows shear thinning behavior. To isolate the rheological effect of the nanowires, the SSF test also was done on a solution of $2.5 \% \mathrm{CMC}$ and $3 \%$ dispersant to make sure that there is no shear thinning effect dictated by rheological agents other than the nanowires. The SSF test result for solution of $2.5 \% \mathrm{CMC}$ and $3 \%$ dispersant is shown in 
Figure 4. 25. Solutions of solvent, binder, and dispersant do not show any shear thinning behavior in the absence of silver nanostructures.

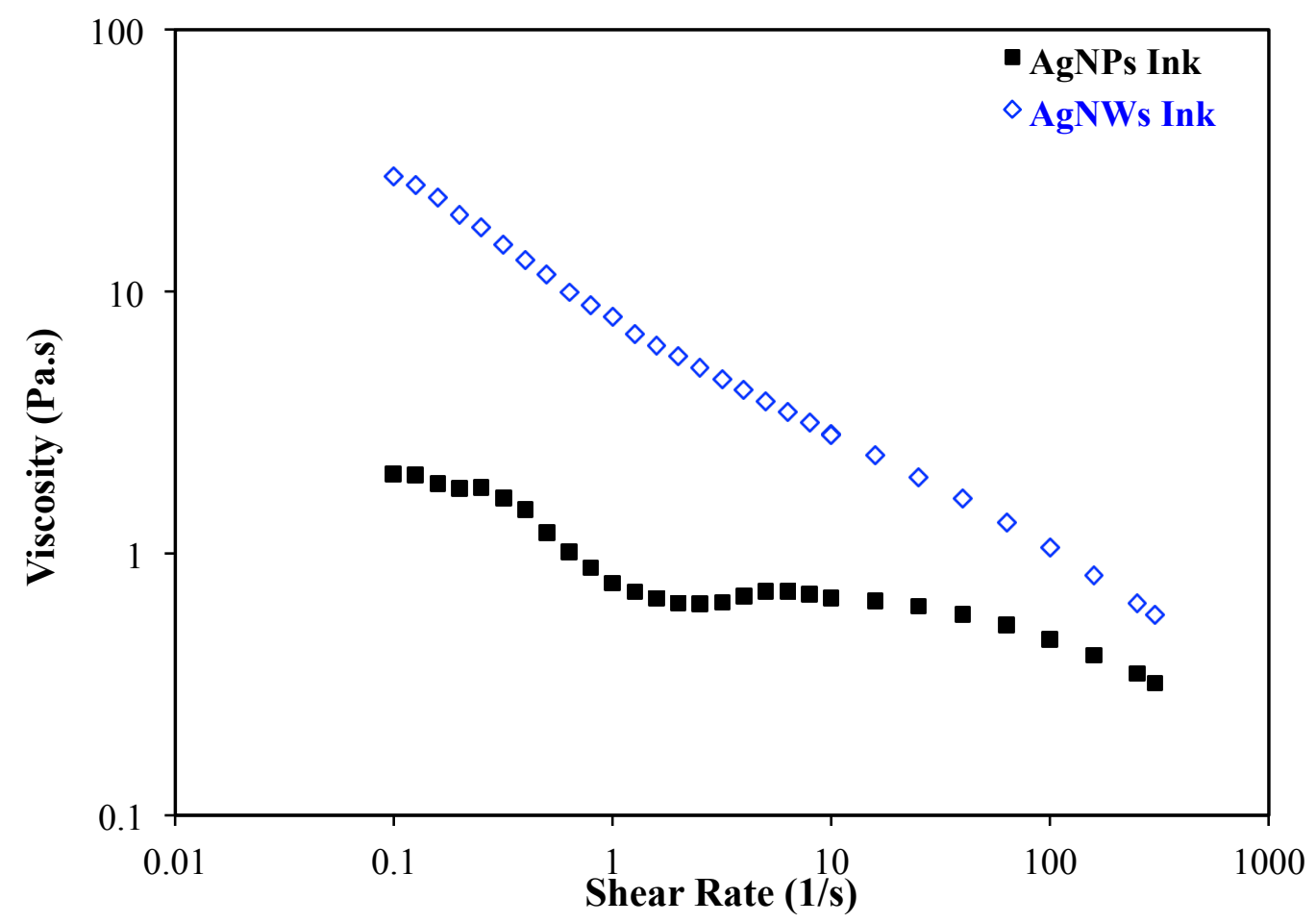

Figure 4. 23: Viscosity versus shear rate for nanoparticle and nanowire suspensions obtained in the steady state flow measurements. 


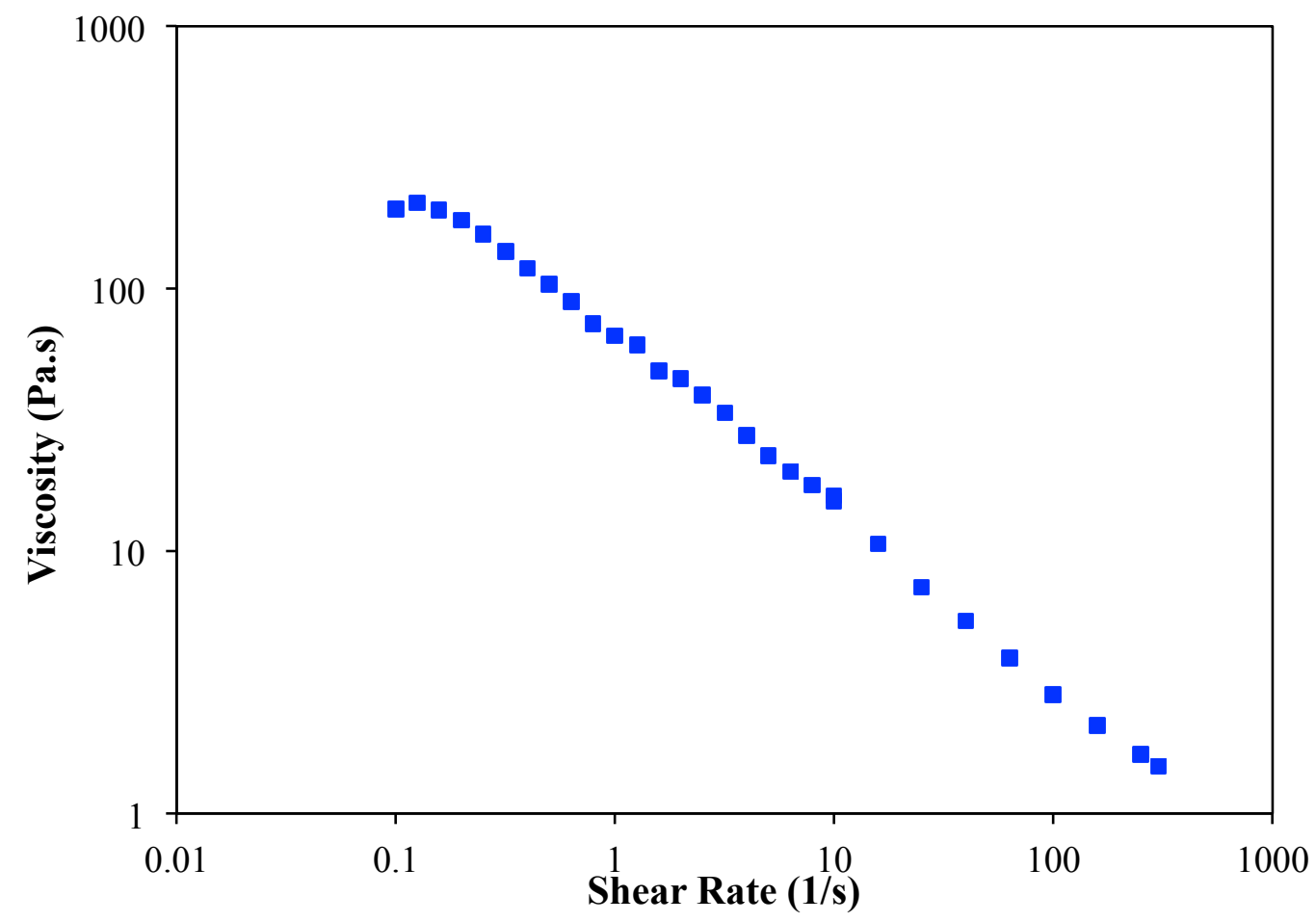

Figure 4. 24: Viscosity versus shear rate for WB-101 ink obtained in the steady state flow measurement.

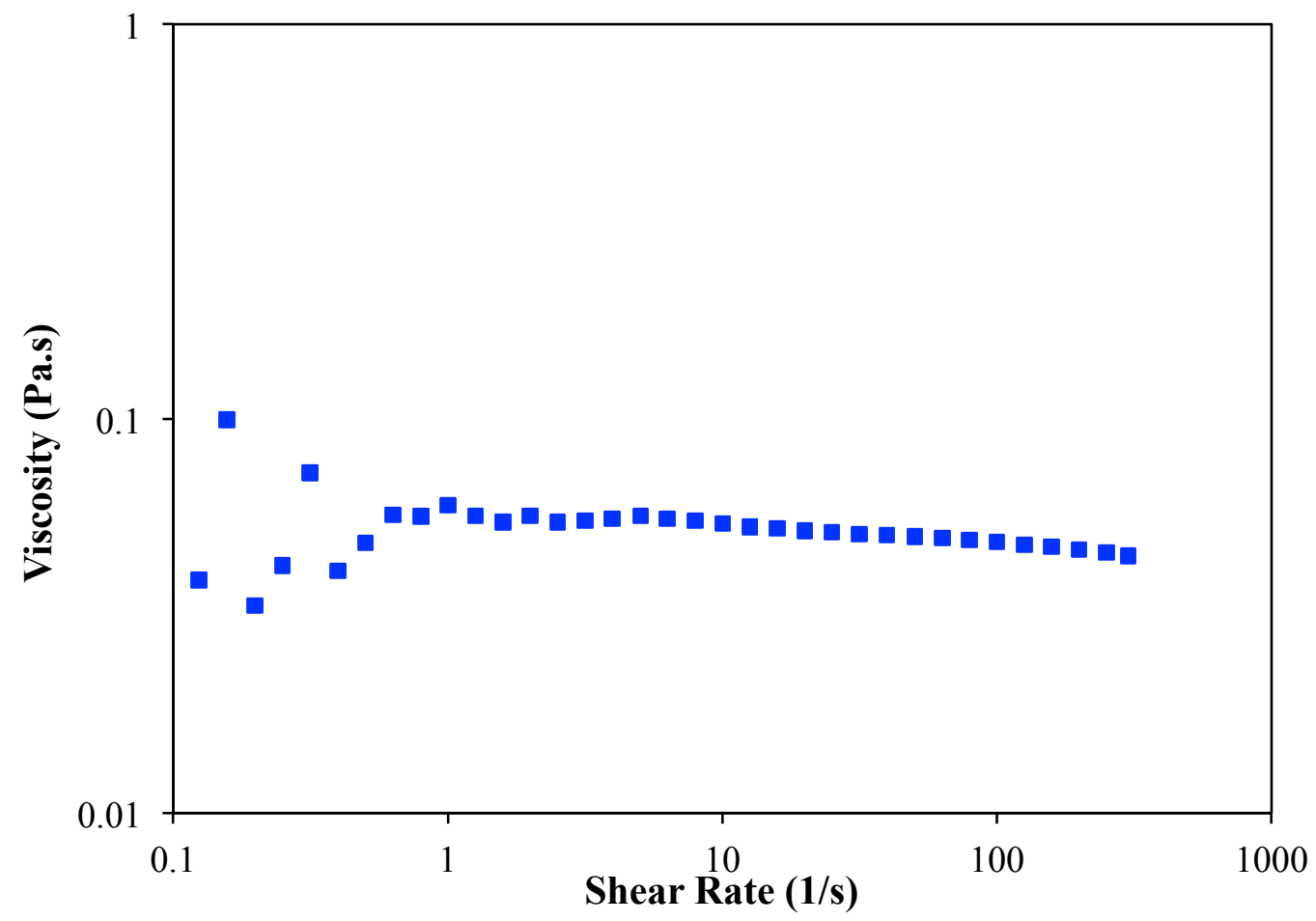

Figure 4. 25: Viscosity versus shear rate for solution of $2.5 \% \mathrm{CMC}$ and $3 \%$ dispersant obtained in the steady state flow measurement. 


\subsubsection{Frequency Sweep Test (FS Test)}

After the LVR determination, the ink was further characterized by a Frequency Sweep (FS) experiment at a stress below the critical stress to determine the variation of $\mathrm{G}^{\prime}$ and $\mathrm{G}^{\prime \prime}$ as functions of angular frequency. The FS test provides more information about the effect of colloidal forces, and the interactions between particles. The results of the FS test for $50 \mathrm{wt} \%$ synthesized AgNWs, and 50 wt.\% AgNPs is shown in Figure 4. 26, and the FS results of the WB-101 ink are shown in Figure 4. 27. Based on the FS test results (see Figure 4. 26, and Figure 4. 27), the G' and $\mathrm{G}^{\prime \prime}$ values increased with increasing angular frequency. The comparison between the rheological results for nanowire and nanoparticle ink illustrates that both $\mathrm{G}^{\prime}$, and $\mathrm{G}^{\prime \prime}$ are larger for silver nanowire ink than for silver nanoparticle ink.

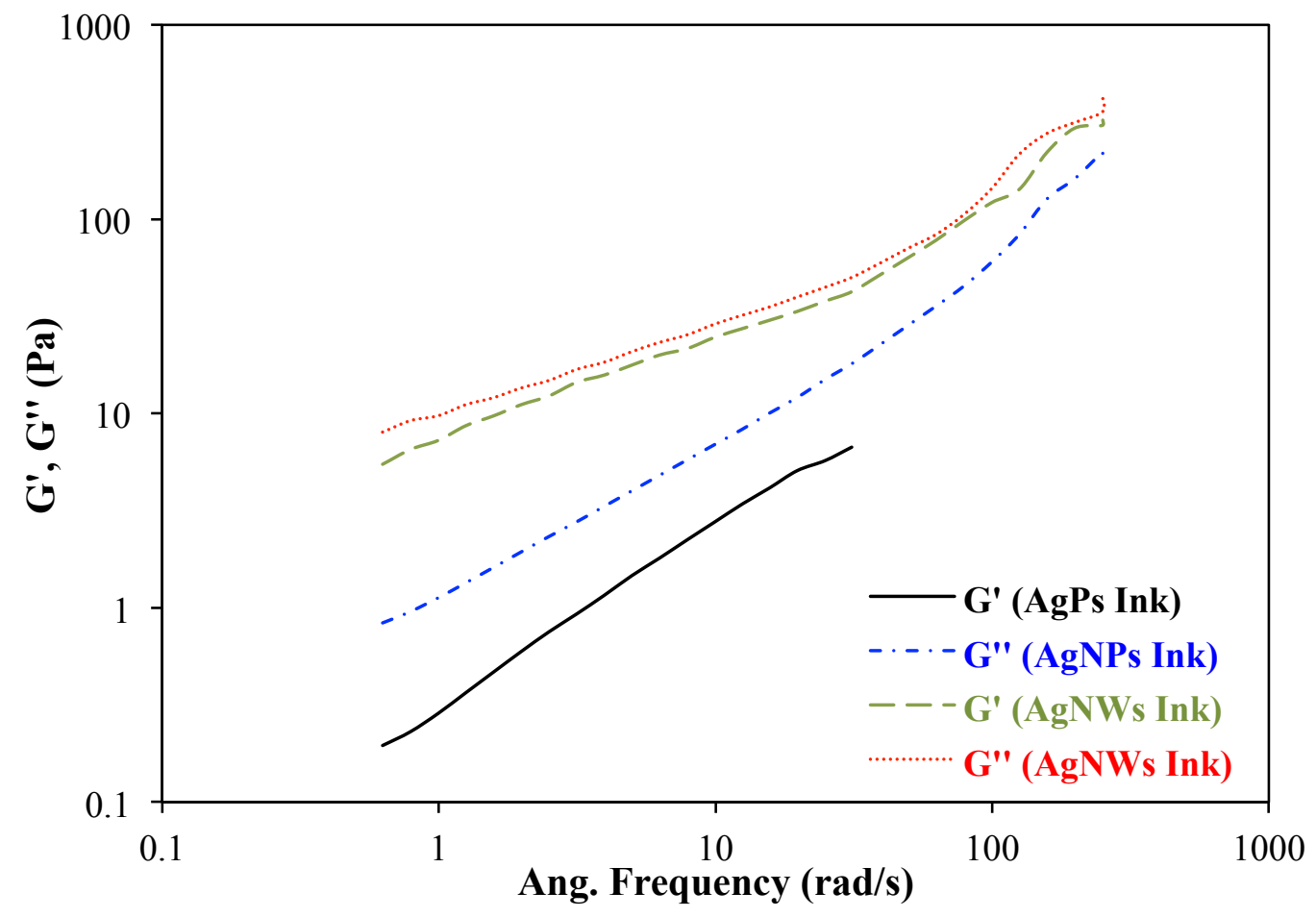

Figure 4. 26: $\mathrm{G}^{\prime}$ and $\mathrm{G}^{\prime \prime}$ versus angular frequency for nanoparticle and nanowire suspensions obtained in the frequency sweep measurements. 


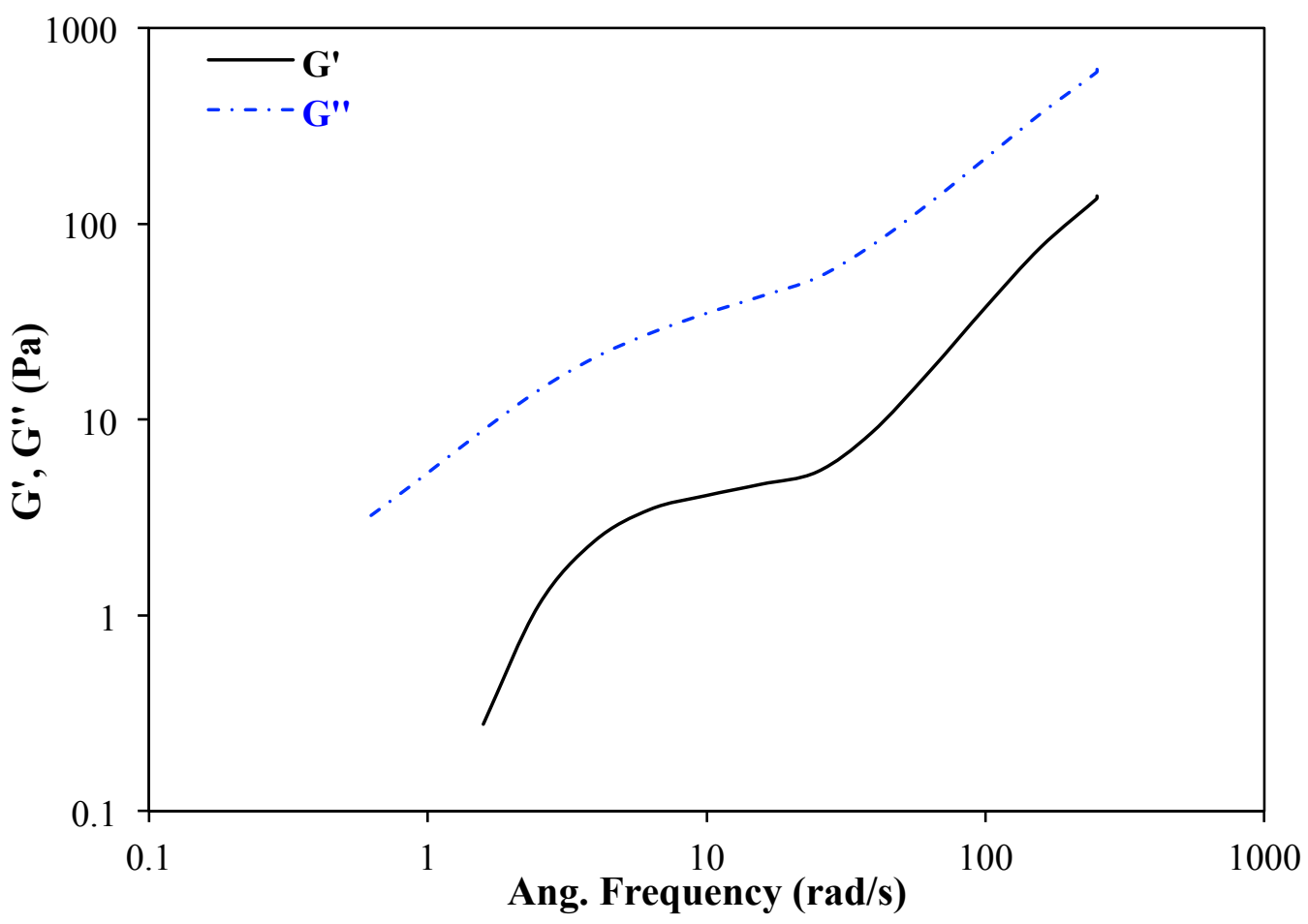

Figure 4. 27: G' and G"versus angular frequency for WB-101 ink obtained in the frequency sweep measurement.

\subsubsection{Peak-Hold Test (PH Test)}

A Peak-Hold ( $\mathrm{PH})$ test was done to mimic the screen printing process. In order to simulate the screen printing process, constant shear rates were applied in three time intervals. The first step was shearing at $0.11 / \mathrm{s}$ for $30 \mathrm{~s}$ to resemble the charging of the ink onto the mesh before printing. The second was shearing at $200 \mathrm{1} / \mathrm{s}$ for $30 \mathrm{~s}$ to represent the screen printing application when the ink is forced through the screen at high shear. The last was shearing at $0.11 / \mathrm{s}$ for 2 min to simulate viscosity recovering after printing.

Figure 4. 28 illustrates the PH results for 50 wt.\% synthesized AgNWs, and 50 wt.\% AgNPs. Figure 4. 29 illustrate the PH results for WB-101 ink. According to the PH results (see Figure 4. 28, and Figure 4. 29), during the high shear rate corresponding to printing, the inks viscosity decreases greatly to allow ink to flow through the mesh. The viscosity recovery process is faster for silver nanoparticle, and WB-101 ink compared to the silver nanowire ink. 


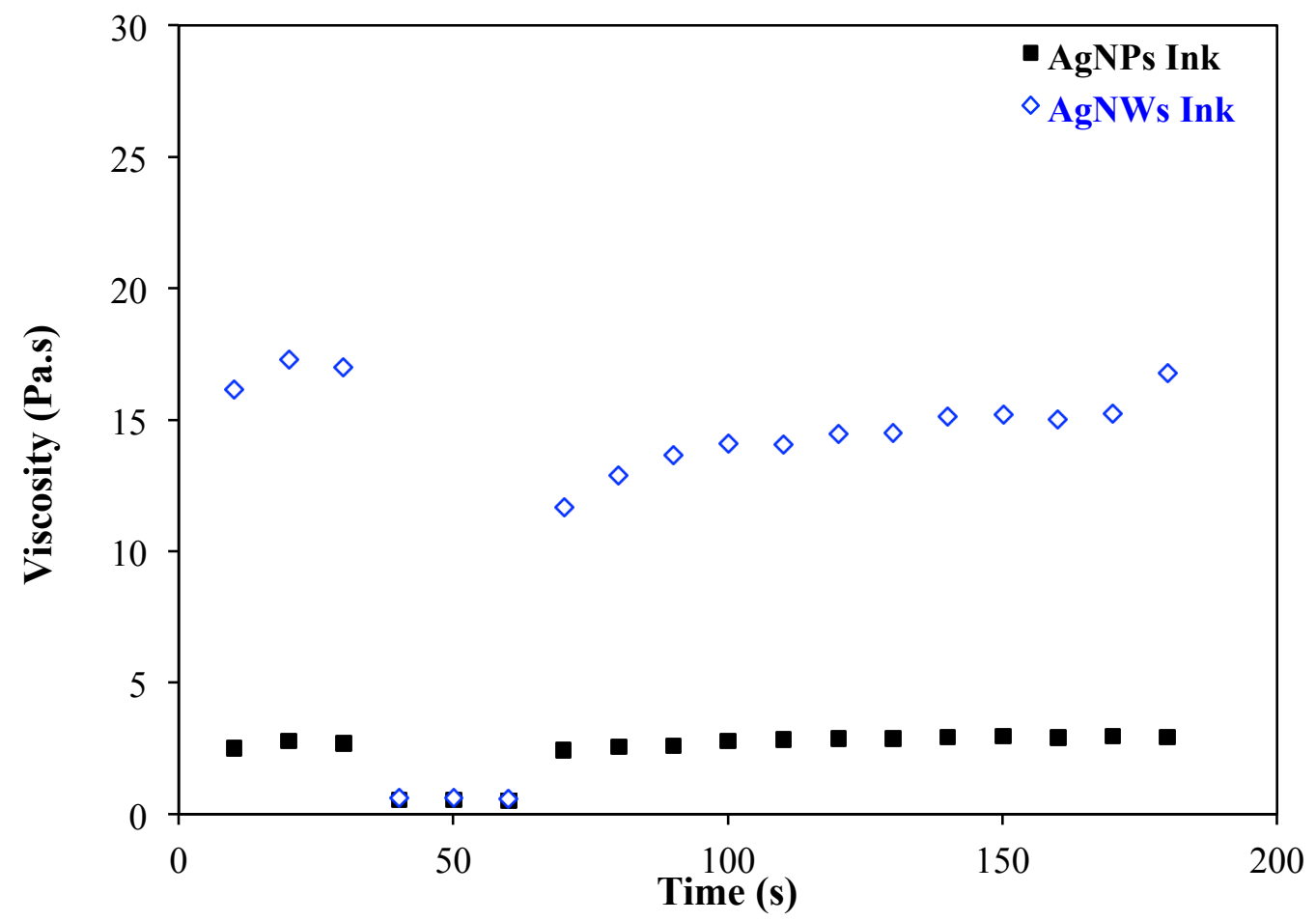

Figure 4. 28: Viscosity versus time for nanoparticle and nanowire suspensions obtained in the peak hold measurements.

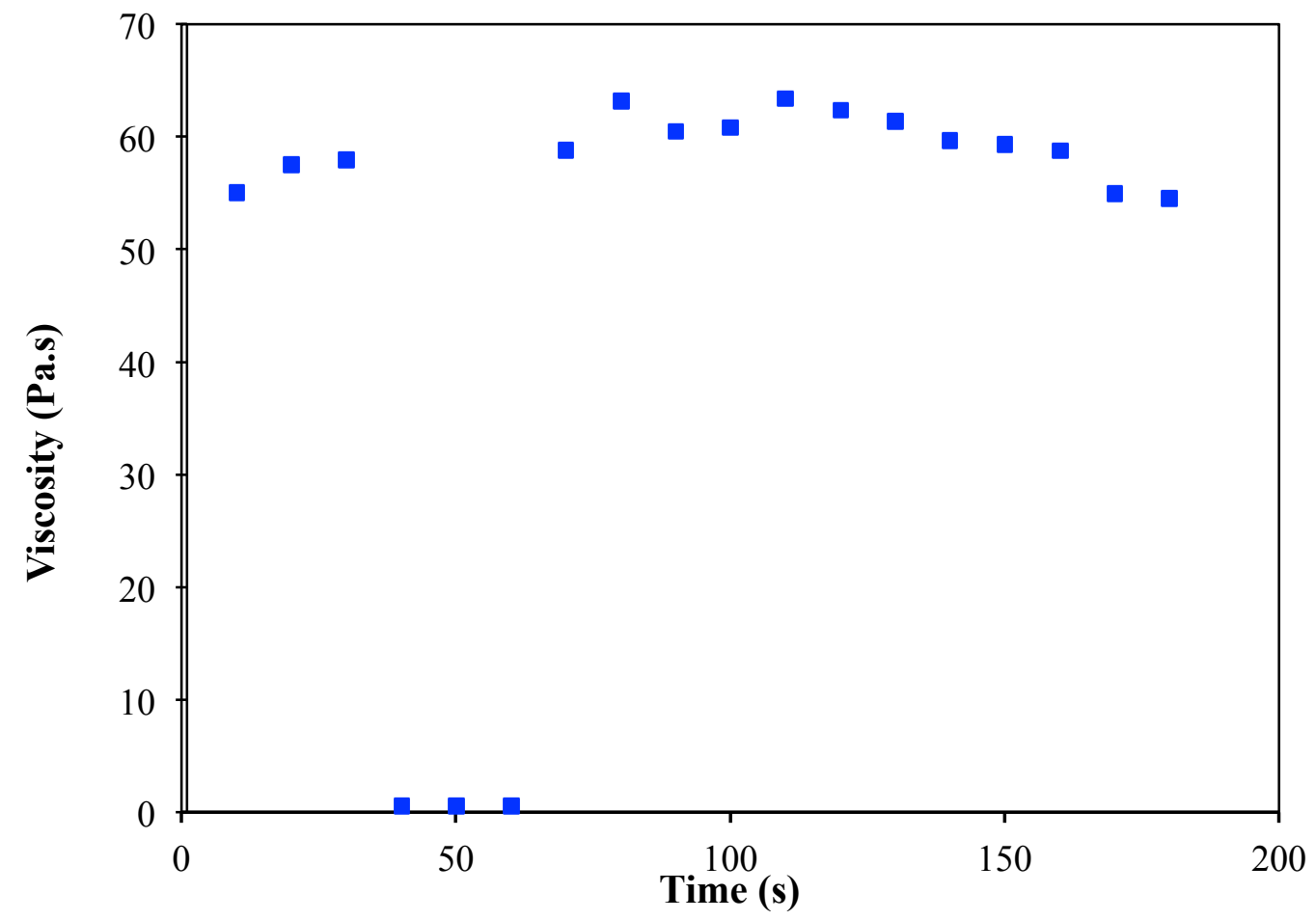

Figure 4. 29: Viscosity versus time for WB-101 ink obtained in the peak hold measurement. 


\subsubsection{Effect of AgNW Content on PH Test Results}

To investigate the effect of silver nanowire content on the rheological behavior of conductive ink during the printing process, the PH test was repeated on inks with silver contents of $20 \%, 30 \%$, $50 \%$, and $60 \%$ by weight. The results are shown in Figure 4.30 and Figure 4.31 . The viscosity depends strongly on AgNWs content at higher weight percents. While the viscosity effect is comparatively small for 20 and $30 \mathrm{wt} . \%$ suspensions, the viscosity is increased by orders of magnitude at concentrations of 50 and $60 \mathrm{wt} . \%$. This behavior is consistent with the formation of entangled networks of wires that impart high viscosity. The wires become disentangled and aligned at high shear rates, and return to the entangled structure after the shear rate is reduced.

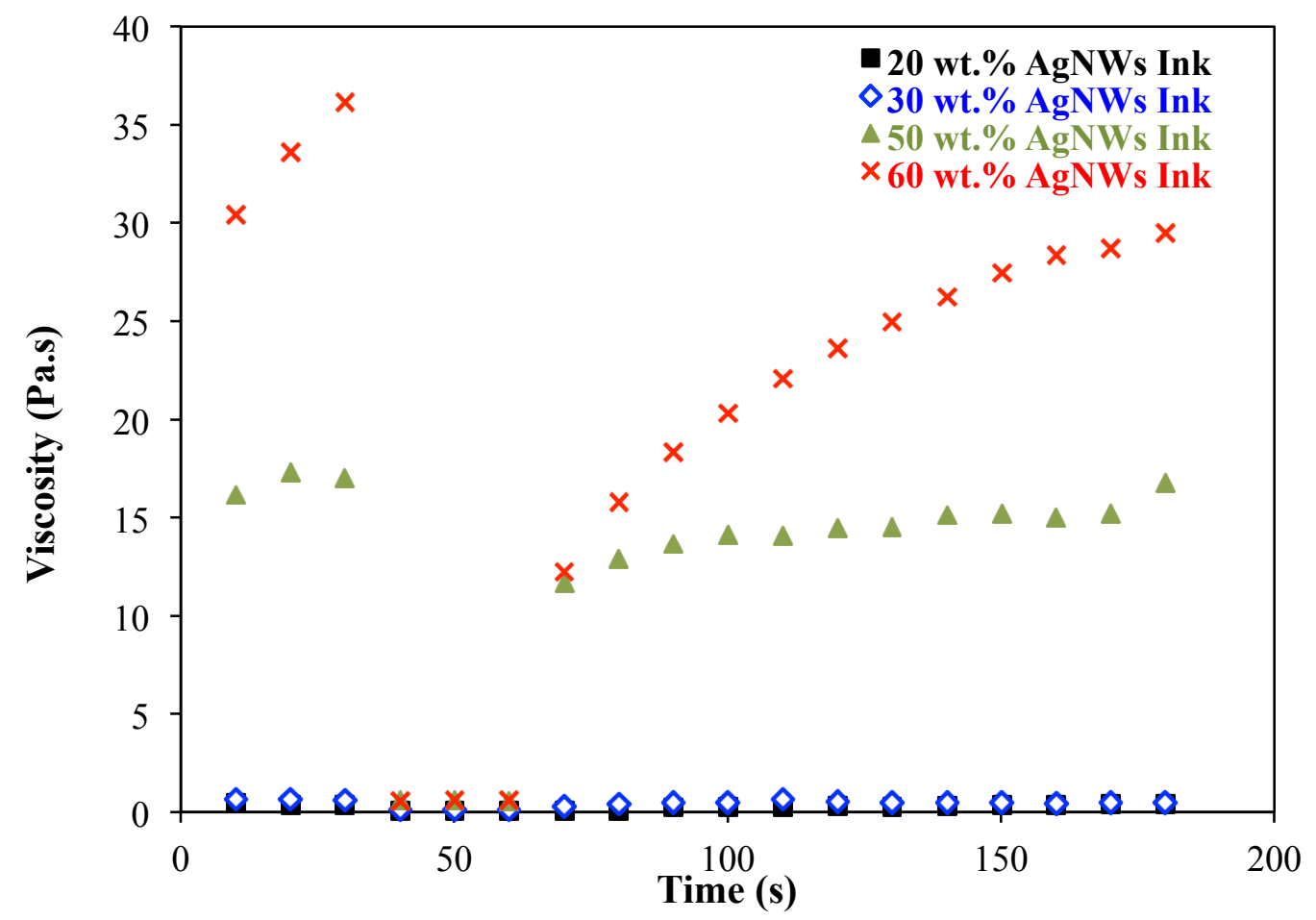

Figure 4. 30: The peak hold measurement repeated at four values of silver nanowire concentration. 


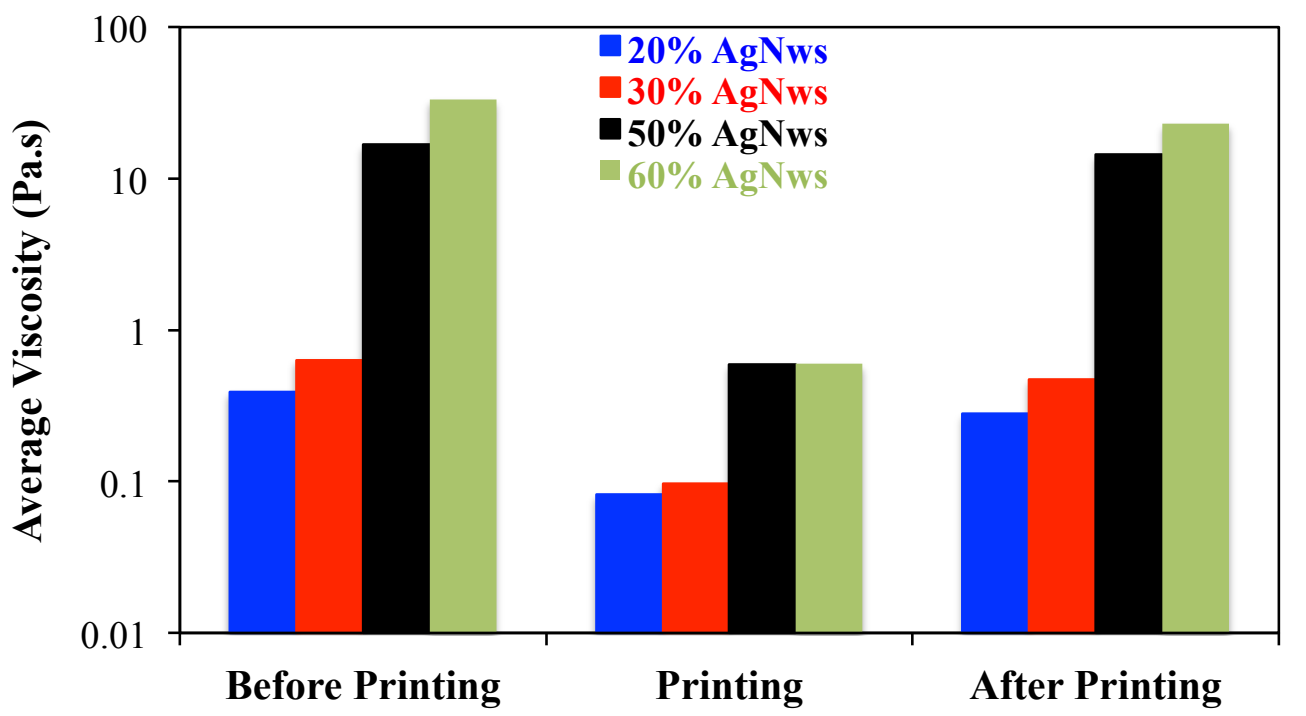

Figure 4. 31: Viscosity at different steps of printing at four values of silver nanowire concentration.

\subsubsection{Comparison between Commercial and Synthesized AgNWs}

In order to examine the effect of the diameter and length of the AgNWs on the rheological behavior of the ink, commercial AgNWs (AgNWs-90-Blue Nano) were utilized to formulate conductive ink. The PH test was done on the same concentration of commercial and synthesized AgNWs. Figure 4. 32 shows the result of the PH test on 5 wt.\% commercial and synthesized AgNWs ink. It is clear that the same concentration of commercial and synthesized AgNWs inks shows different rheological behavior. The average diameter of both synthesized and commercial AgNWs are about $90 \mathrm{~nm}$. The average length of synthesized AgNWs is about $2 \mu \mathrm{m}$, and the average length of commercial AgNWs is about $30 \mu \mathrm{m}$. The longer wires can change the viscosity of the ink by order of magnitude, and rheological behavior during the screen printing process. 


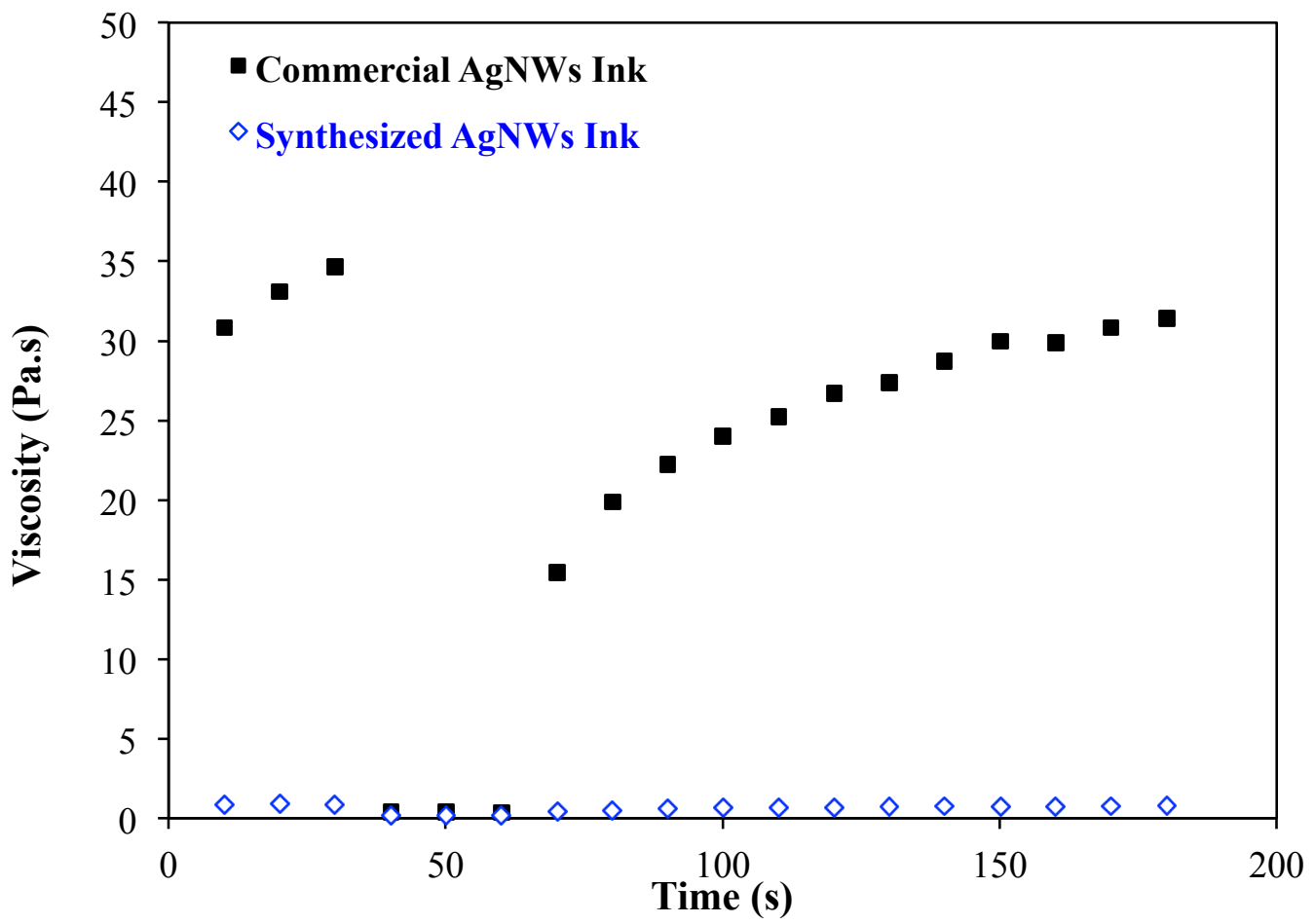

Figure 4. 32: The peak hold measurements for commercial and synthesized AgNWs ink.

\subsubsection{Rheological Behavior of Commercial AgNW Ink during Screen Printing}

Water-based ink was formulated with commercial AgNWs (AgNWs-90-Blue Nano, and AgNWs-40 Blue Nano), as conductive or metal filler, deionized (DI) water and Isopropyl Alcohol ((IPA), PHARMCO-AAPER, 231HPLC99) as solvent or carrier, Carboxymethyl Cellulose (CMC, Sigma- Aldrich, 419237) as a binder or thickener, and Dispex Ultra FA 4416 (Old Hydropalat 216), BASF) as a dispersive agent. Inks with AgNWs-90 contents of 3\%, 4\%, $5 \%, 6 \%$, and $7 \%$ by weight were prepared. To investigate the effect of the AgNW dimensions on the rheological behavior of the inks, several AgNWs (AgNWs-40-Blue Nano) ink content of $0.04 \%, 0.25 \%, 0.5 \%, 0.75 \%, 1 \%, 1.25 \%, 1.5 \%, 3 \%, 4 \%, 5 \%$, and $6 \%$ by weight were prepared. In order to investigate the effect of temperature on the build-up of the ink structure after screen printing, the PH test was repeated for each ink at temperatures of $288 \mathrm{~K}, 298 \mathrm{~K}, 308 \mathrm{~K}$, and 318 
K. Moreover the PH test was repeated at lower recovery shear rate $(0.041 / \mathrm{s})$ to investigate the effect of the lower shearing on the build-up and viscosity recovery mechanisms.

\subsubsection{Simulation of Screen Printing (PH Test)}

Figure 4. 33 shows the results of the PH test on different commercial AgNWs (AgNWs-90) inks at several temperatures. Similar results are shown for AgNWs-40 inks in Figure 4. 34. Figure 4. 35 shows the results of the PH test at different values of shearing during the recovery process after screen printing for AgNWs-90.

Based on the PH tests on different inks with different silver nanowire contents, the viscosity depends strongly on AgNWs content. This effect is stronger at higher concentration of AgNWs. At any concentration of AgNWs, the viscosity is higher at lower temperature. Moreover, the viscosity is larger at lower shear rate recovery, which is corresponds to the shear thinning thixotropic behavior of the ink. Comparison between viscosities of the ink during different steps of the screen printing process illustrates that the prepared ink with lower diameter of AgNWs has higher viscosity. 

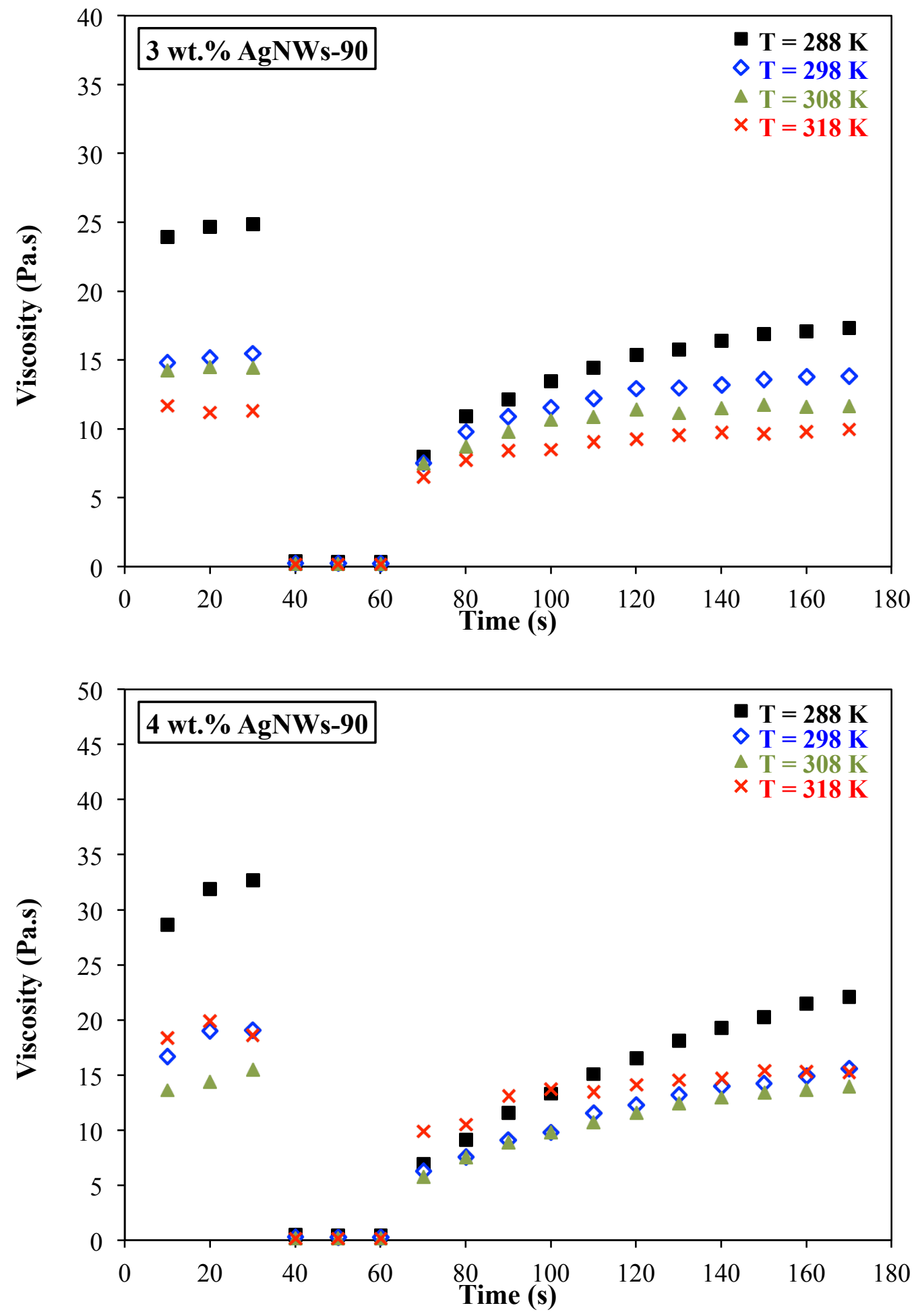

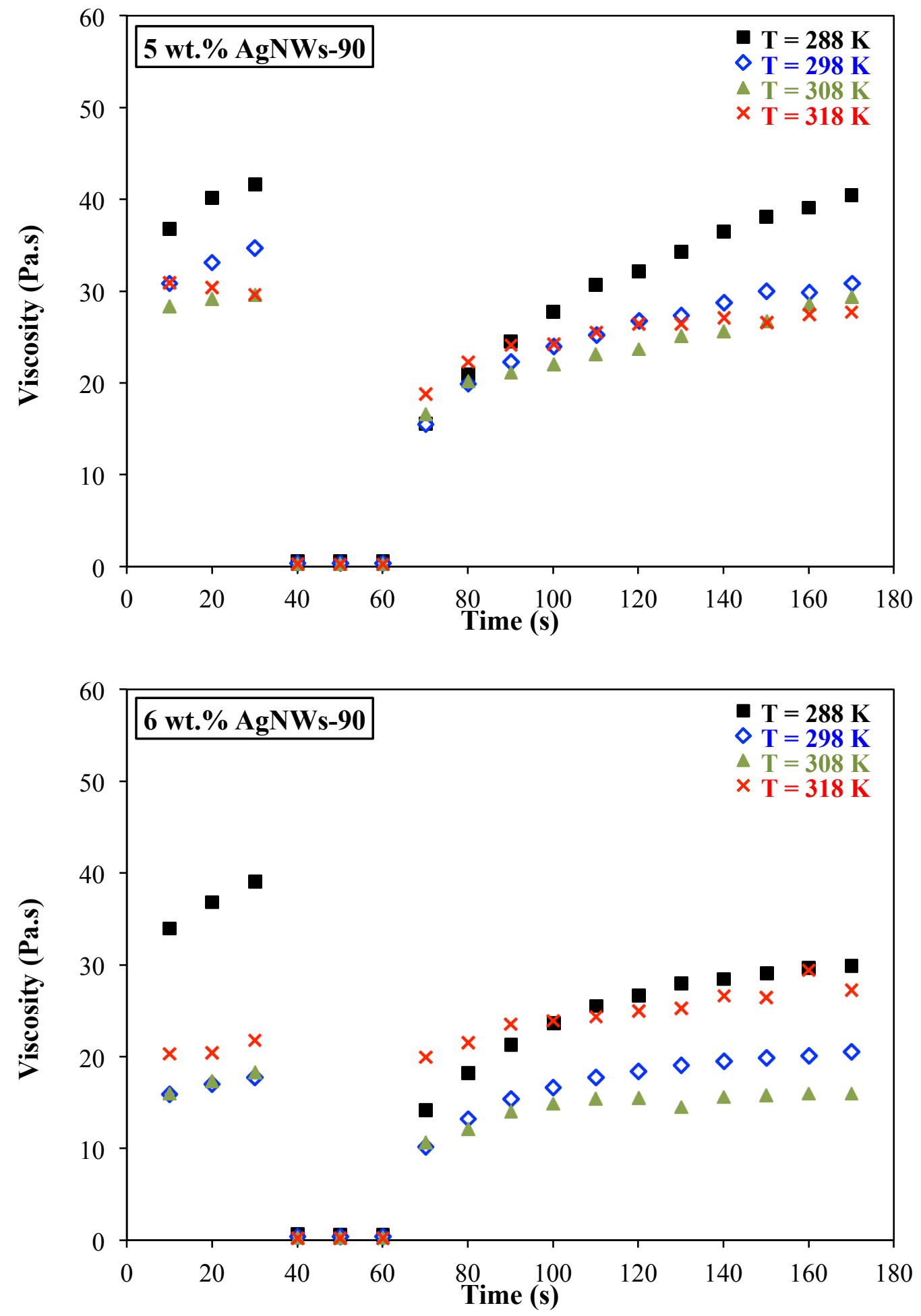


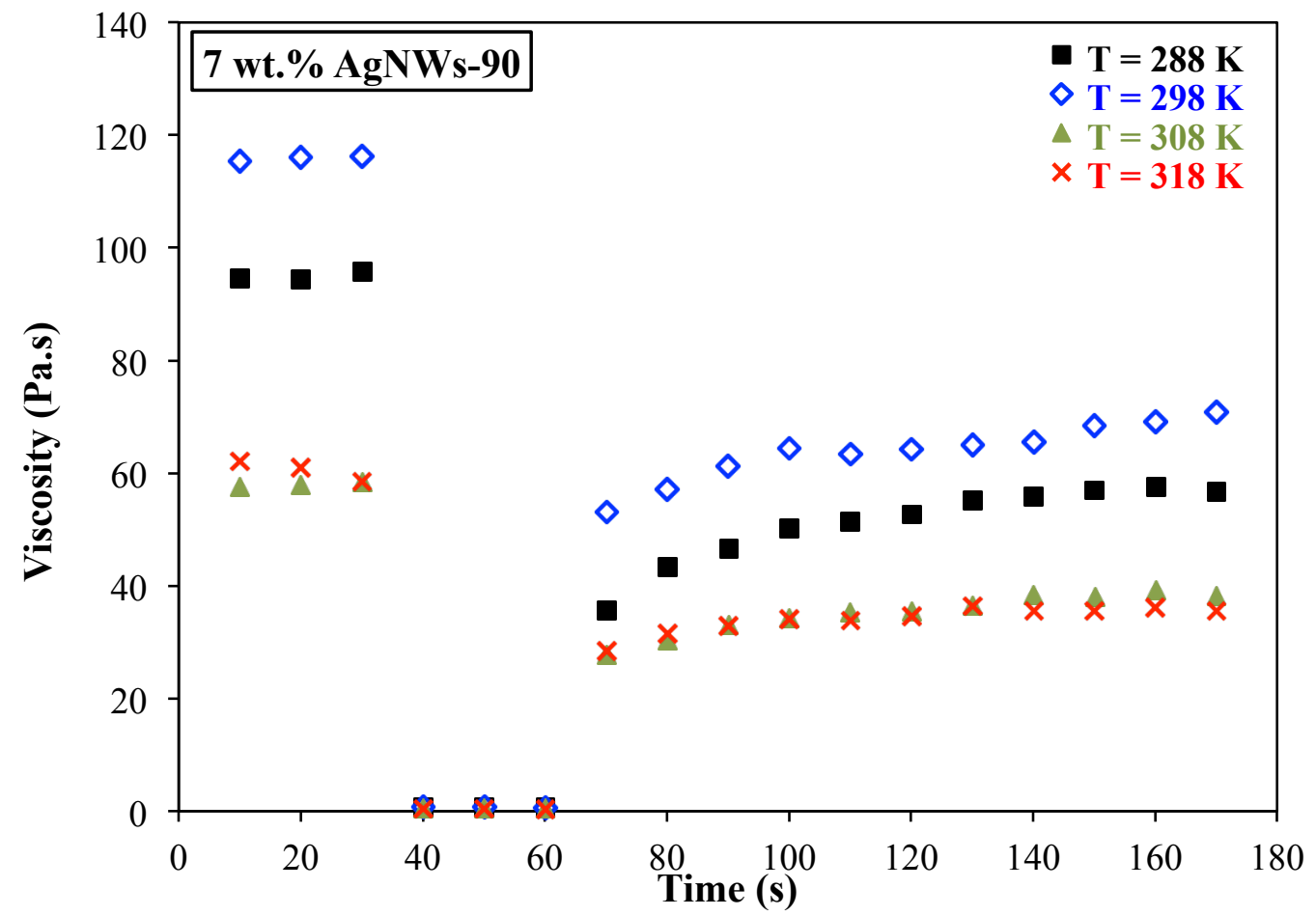

Figure 4. 33: Measured viscosity versus time in the PH test for several contents of AgNWs (AgNWs-90) ink.

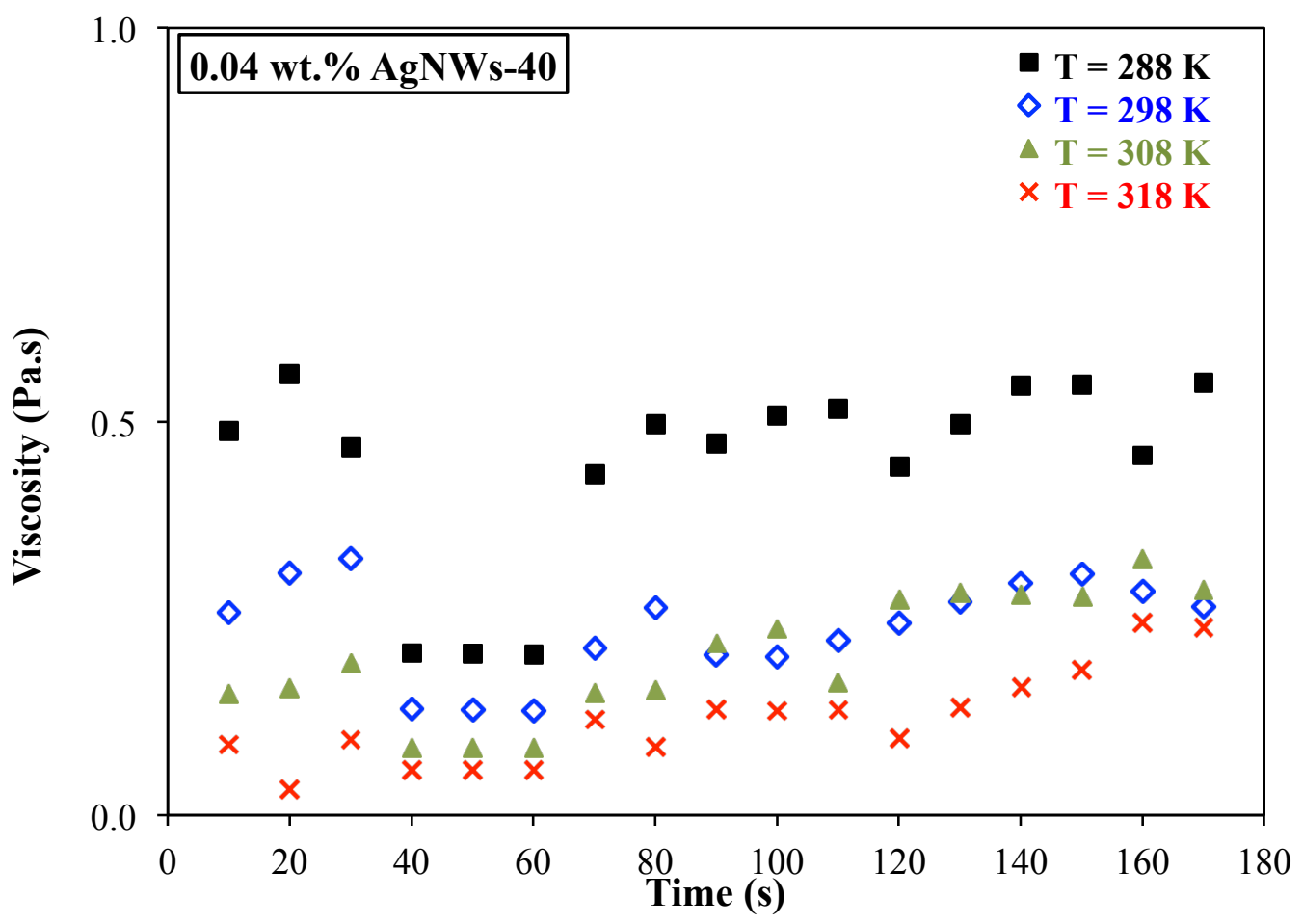



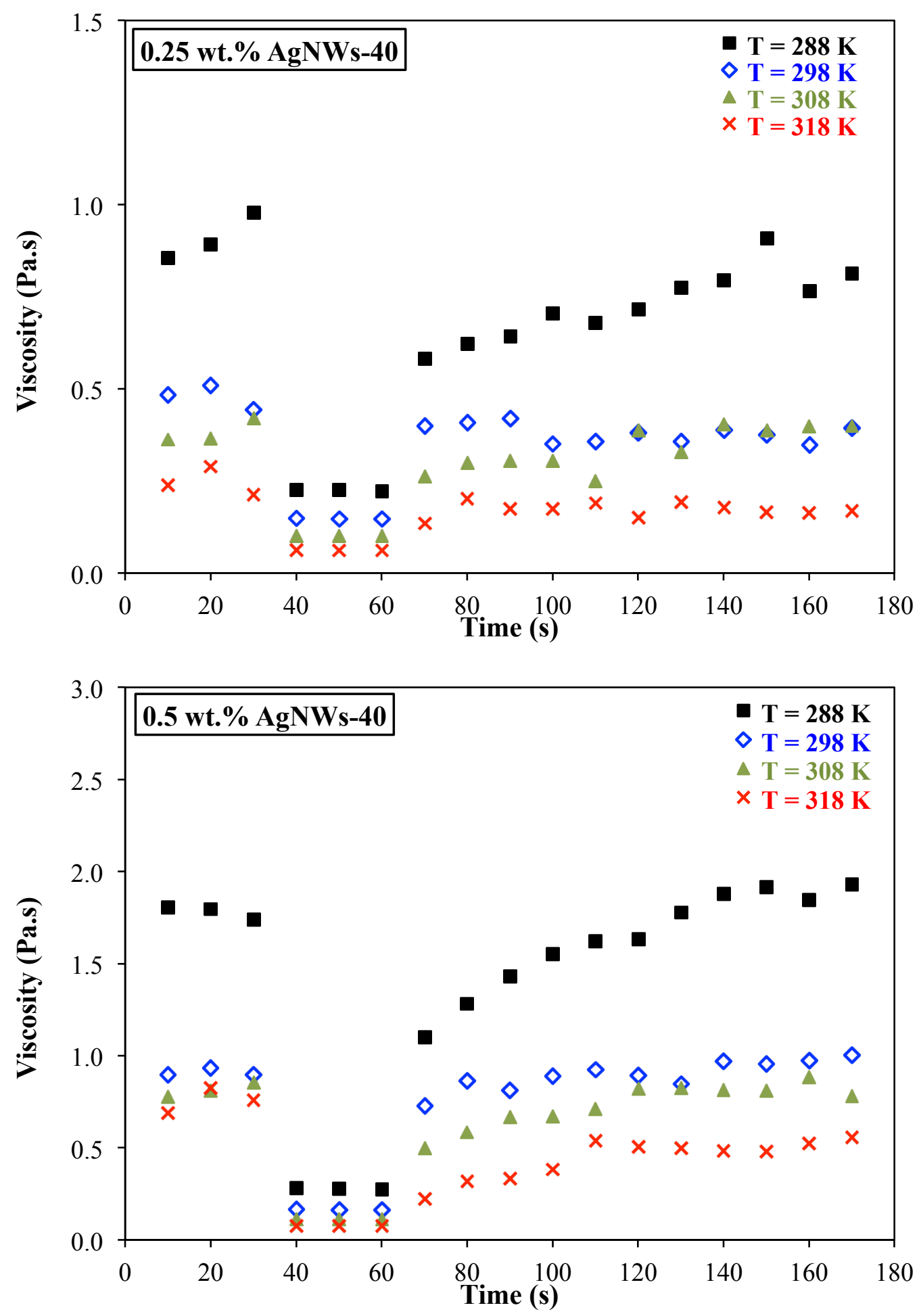

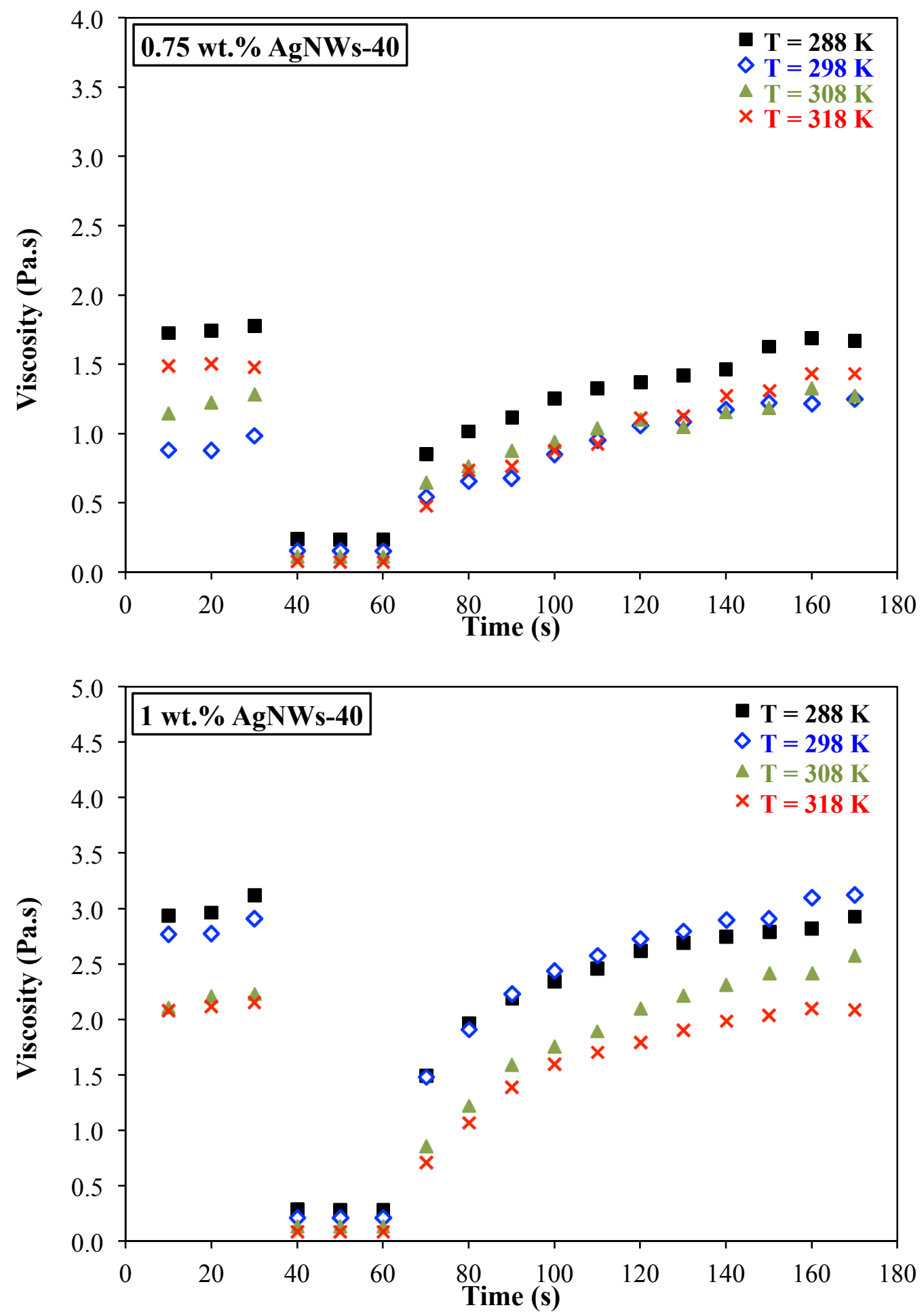

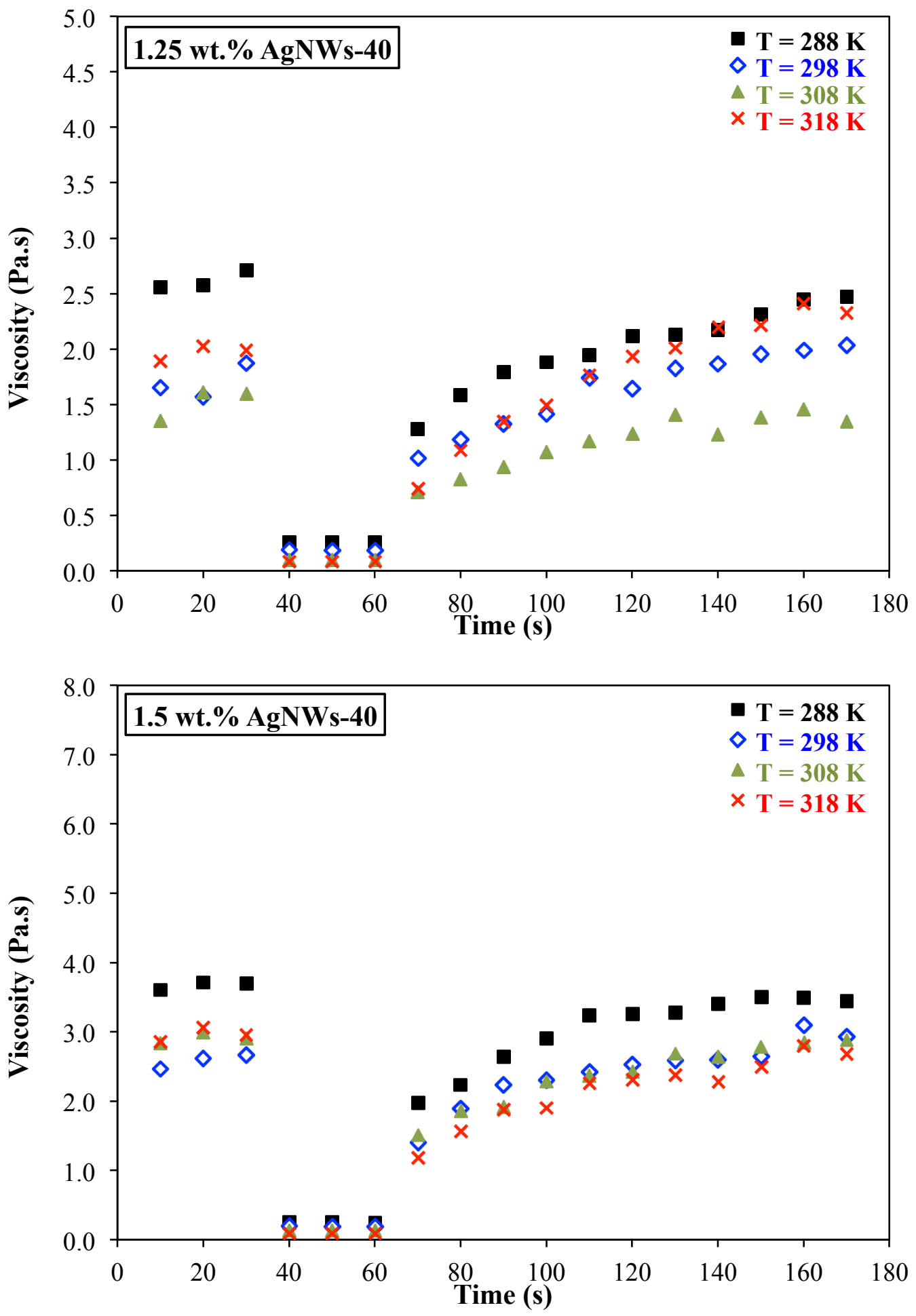

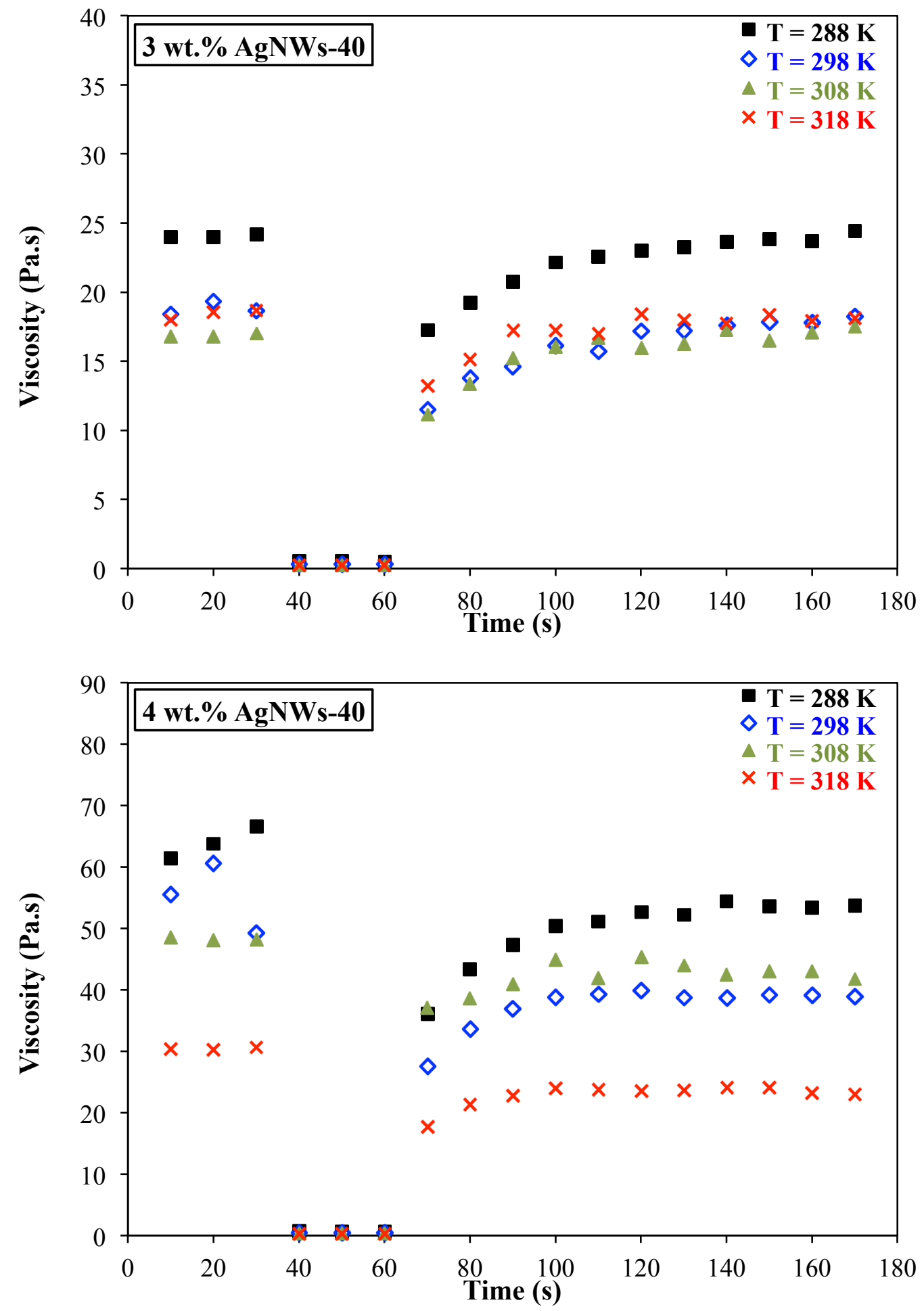

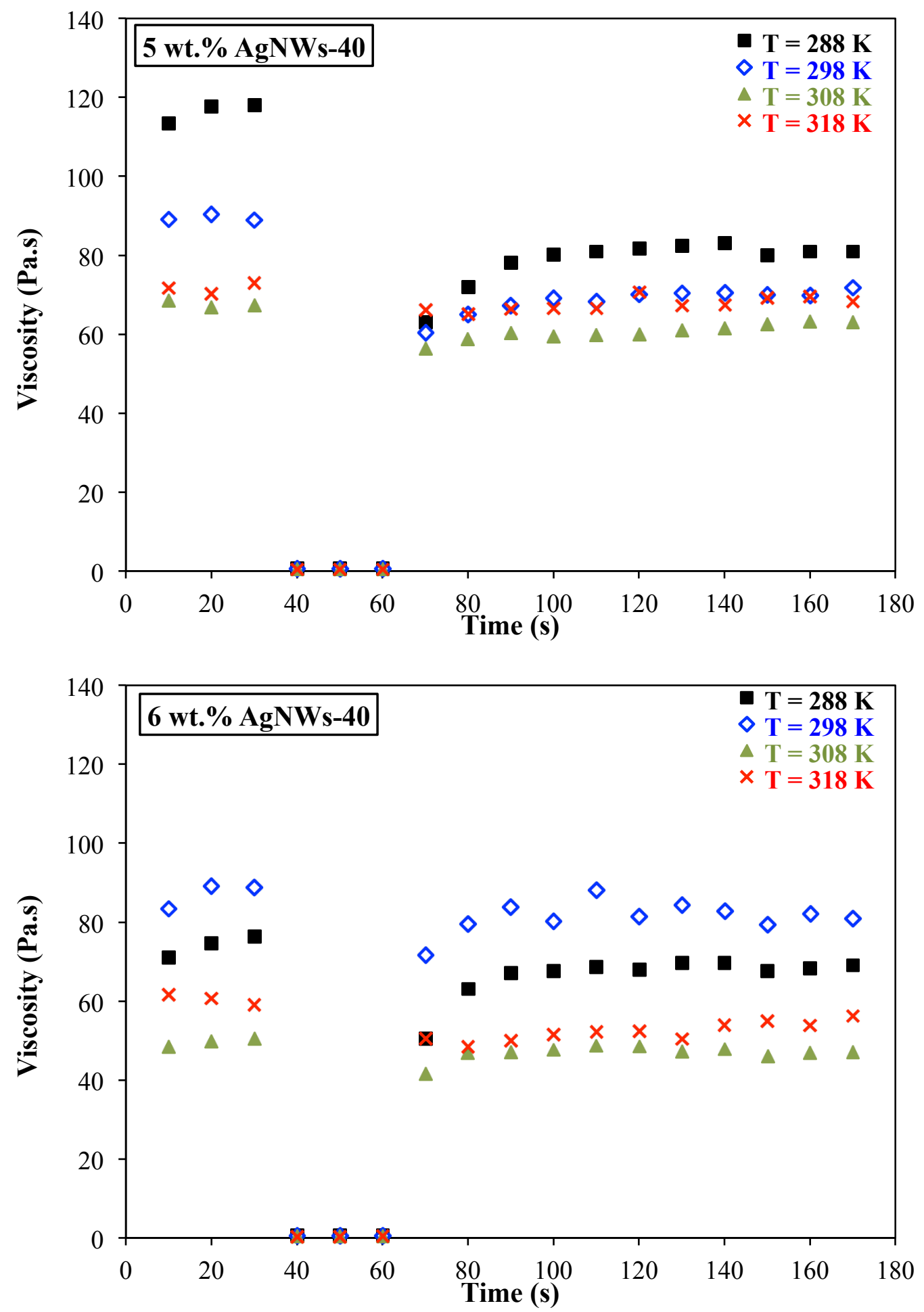

Figure 4. 34: Measured viscosity versus time in the PH test for several contents of AgNWs (AgNWs-40) ink. 

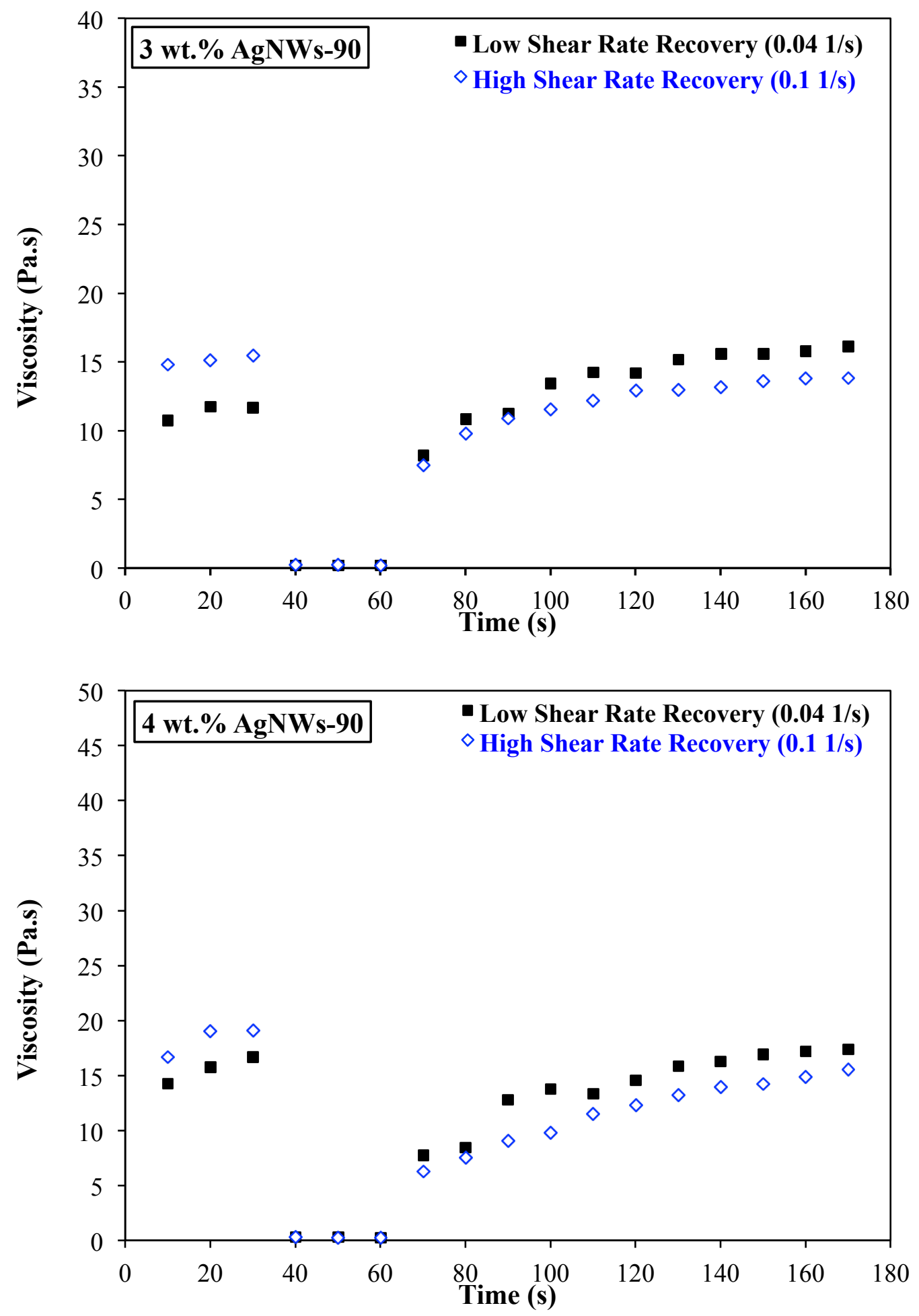

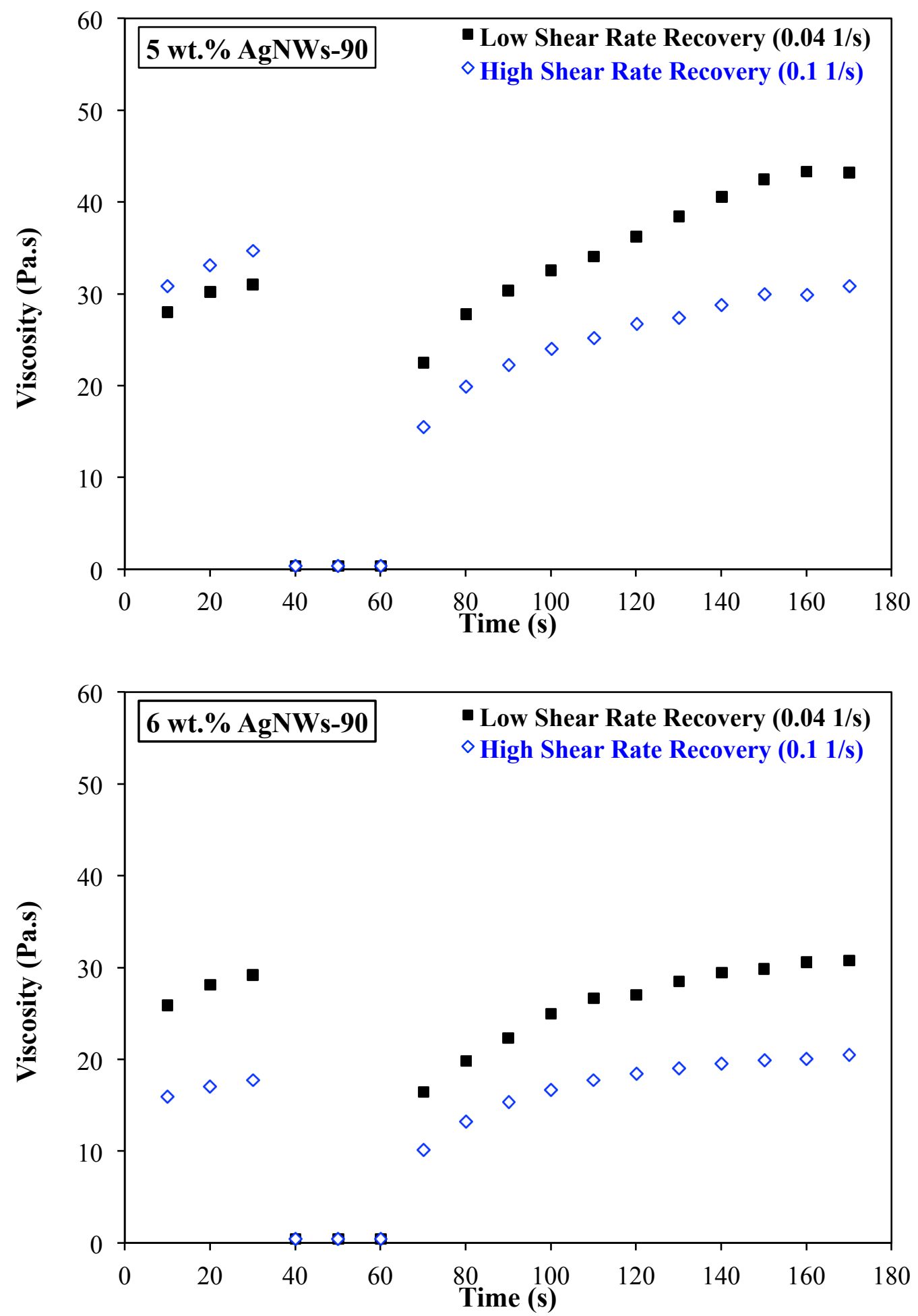

Figure 4. 35: Measured viscosity versus time in the PH test for different AgNWs (AgNWs-90) contents at two values of shearing in viscosity recovery. 
The results for the PH tests are summarized in Table 4. 3, and Table 4. 4 for AgNWs-90 at several temperatures, and at low recovery shear rate, respectively. The results for the $\mathrm{PH}$ tests are summarized in Table 4. 5 for AgNWs-40 at several temperatures.

Table 4. 3: Average viscosity at different steps of the peak hold test for different silver nanowire (AgNWs-90) contents at several temperatures.

\begin{tabular}{|c|c|c|c|c|}
\hline \multirow{2}{*}{ Sample } & \multirow{2}{*}{ Temperature (K) } & \multicolumn{3}{|c|}{ Viscosity (Pa.s) } \\
\hline & & Average at Low Shear & Average at High Shear & Final Recovered \\
\hline \multirow{4}{*}{4 wt. $\%$ AgNW-90 } & 288 & 31.0 & 0.45 & 22.8 \\
\hline & 298 & 18.3 & 0.26 & 15.6 \\
\hline & 308 & 14.5 & 0.21 & 14.2 \\
\hline & 318 & 19.0 & 0.16 & 16.1 \\
\hline \multirow{4}{*}{5 wt.\% AgNW-90 } & 288 & 39.5 & 0.57 & 41.5 \\
\hline & 298 & 32.9 & 0.38 & 31.4 \\
\hline & 308 & 29.0 & 0.31 & 27.5 \\
\hline & 318 & 30.3 & 0.26 & 27.7 \\
\hline \multirow{4}{*}{6 wt.\% AgNW-90 } & 288 & 36.6 & 0.63 & 30.2 \\
\hline & 298 & 16.9 & 0.41 & 20.3 \\
\hline & 308 & 17.2 & 0.28 & 16.0 \\
\hline & 318 & 20.8 & 0.22 & 27.3 \\
\hline \multirow{4}{*}{7 wt.\% AgNW-90 } & 288 & 94.8 & 0.69 & 58.5 \\
\hline & 298 & 116 & 0.63 & 68.7 \\
\hline & 308 & 57.9 & 0.39 & 38.2 \\
\hline & 318 & 60.6 & 0.34 & 35.8 \\
\hline
\end{tabular}

Table 4. 4: Average viscosity at different steps of the peak hold test for different silver nanowire (AgNWs-90) contents at two values of recovery shear rate.

\begin{tabular}{|c|c|c|c|c|}
\hline \multirow{2}{*}{ Sample } & \multirow{2}{*}{ Shear Rate (1/s) } & \multicolumn{3}{|c|}{ Viscosity (Pa.s) } \\
\cline { 3 - 5 } & & Average at Low Shear & Average at High Shear & Final Recovered \\
\hline \multirow{2}{*}{ 4 wt.\% AgNW-90 } & 0.04 & 15.6 & 0.27 & 18.1 \\
\cline { 2 - 5 } & 0.1 & 18.3 & 0.26 & 15.6 \\
\hline \multirow{2}{*}{ 5 wt.\% AgNW-90 } & 0.04 & 29.8 & 0.37 & 43.7 \\
\cline { 2 - 5 } & 0.1 & 32.9 & 0.38 & 31.4 \\
\hline \multirow{2}{*}{ 6 wt.\% AgNW-90 } & 0.04 & 27.7 & 0.42 & 30.7 \\
\cline { 2 - 5 } & 0.1 & 16.9 & 0.41 & 20.3 \\
\hline
\end{tabular}


Table 4. 5: Average viscosity at different steps of the peak hold test for different silver nanowire (AgNWs-40) contents at several temperatures.

\begin{tabular}{|c|c|c|c|c|}
\hline \multirow{2}{*}{ Sample } & \multirow{2}{*}{ Temperature (K) } & \multicolumn{3}{|c|}{ Viscosity (Pa.s) } \\
\hline & & Average at Low Shear & Average at High Shear & Final Recovered \\
\hline \multirow{4}{*}{0.04 wt. $\%$ AgNW-40 } & 288 & 0.50 & 0.20 & 0.56 \\
\hline & 298 & 0.29 & 0.13 & 0.32 \\
\hline & 308 & 0.17 & 0.08 & 0.25 \\
\hline & 318 & 0.07 & 0.05 & 0.21 \\
\hline \multirow{4}{*}{0.25 wt. $\%$ AgNW-40 } & 288 & 0.91 & 0.22 & 0.82 \\
\hline & 298 & 0.48 & 0.15 & 0.47 \\
\hline & 308 & 0.38 & 0.10 & 0.42 \\
\hline & 318 & 0.25 & 0.06 & 0.18 \\
\hline \multirow{4}{*}{0.5 wt. $\%$ AgNW-40 } & 288 & 1.78 & 0.28 & 1.94 \\
\hline & 298 & 0.91 & 0.16 & 0.95 \\
\hline & 308 & 0.81 & 0.11 & 0.80 \\
\hline & 318 & 0.76 & 0.08 & 0.64 \\
\hline \multirow{4}{*}{0.75 wt. $\%$ AgNW-40 } & 288 & 1.75 & 0.24 & 1.71 \\
\hline & 298 & 0.91 & 0.15 & 1.24 \\
\hline & 308 & 1.21 & 0.11 & 1.33 \\
\hline & 318 & 1.49 & 0.07 & 1.41 \\
\hline \multirow{4}{*}{1 wt. $\%$ AgNW-40 } & 288 & 3.00 & 0.28 & 2.95 \\
\hline & 298 & 2.82 & 0.21 & 3.17 \\
\hline & 308 & 2.18 & 0.13 & 2.59 \\
\hline & 318 & 2.11 & 0.08 & 2.27 \\
\hline \multirow{4}{*}{1.25 wt. $\%$ AgNW-40 } & 288 & 2.62 & 0.26 & 2.54 \\
\hline & 298 & 1.70 & 0.18 & 2.15 \\
\hline & 308 & 1.52 & 0.09 & 1.70 \\
\hline & 318 & 1.97 & 0.08 & 1.84 \\
\hline \multirow{4}{*}{1.5 wt. $\%$ AgNW-40 } & 288 & 3.67 & 0.25 & 3.34 \\
\hline & 298 & 2.58 & 0.19 & 2.85 \\
\hline & 308 & 2.91 & 0.13 & 2.96 \\
\hline & 318 & 2.96 & 0.09 & 2.75 \\
\hline \multirow{4}{*}{3 wt. $\%$ AgNW-40 } & 288 & 24.0 & 0.50 & 24.5 \\
\hline & 298 & 18.8 & 0.32 & 18.5 \\
\hline & 308 & 16.9 & 0.25 & 18.0 \\
\hline & 318 & 18.4 & 0.23 & 18.4 \\
\hline \multirow{4}{*}{4 wt. $\%$ AgNW-40 } & 288 & 63.9 & 0.65 & 53.8 \\
\hline & 298 & 55.1 & 0.47 & 39.2 \\
\hline & 308 & 48.3 & 0.35 & 44.0 \\
\hline & 318 & 30.4 & 0.24 & 23.4 \\
\hline \multirow{4}{*}{5 wt. $\%$ AgNW-40 } & 288 & 116 & 0.71 & 81.2 \\
\hline & 298 & 89.4 & 0.59 & 70.6 \\
\hline & 308 & 67.5 & 0.43 & 63.7 \\
\hline & 318 & 71.6 & 0.34 & 67.8 \\
\hline \multirow{4}{*}{6 wt. $\%$ AgNW-40 } & 288 & 74.0 & 0.67 & 68.4 \\
\hline & 298 & 87.0 & 0.60 & 79.6 \\
\hline & 308 & 49.6 & 0.42 & 47.5 \\
\hline & 318 & 60.5 & 0.33 & 53.0 \\
\hline
\end{tabular}




\subsubsection{Correlation of Build-up Structure (SE model)}

The results for the correlation of the build-up structure during viscosity recovery process based on the SEmo are shown in Figure 4. 36 and Figure 4. 37. Figure 4. 36 shows the results at different temperatures, and Figure 4. 37 shows the results at different shearing values during the viscosity recovery process for AgNWs-90. Figure 4. 38 shows the fit for AgNWs-40 at different temperatures. These results show that the rate of viscosity recovery depends on the silver nanowire content, temperature, and applied shear rate.

Correlation with the stretched exponential model provides a characteristic time for the recovery process. The model is based on the assumption that viscosity recovery proceeds by a transition from a more oriented structure at high shear to a more random structure at low shear. If the structure can be characterized by one parameter that varies smoothly from the high shear value to the low shear value, and the rate of the transition is proportional to the difference between the structural parameter and its equilibrium value at low shear, one obtains the exponential time dependence of the SEmo.

The characteristic time, as a model parameter, is correlated with the shear thinning thixotropic behavior of the AgNWs inks. The characteristic time represents the required time for viscosity recovery and silver nanowire rearrangements after the printing process without ink spreading in order to have sharp line definition of the printed patterns $[35,36]$. In this interpretation, the reciprocal of the time constant is a rate constant. The rate constant is a function of the mechanisms by which the structure becomes randomized and of the thermal energy available to drive the mechanism. Hence, we can treat the rate constant with a conventional Arrhenius plot. 

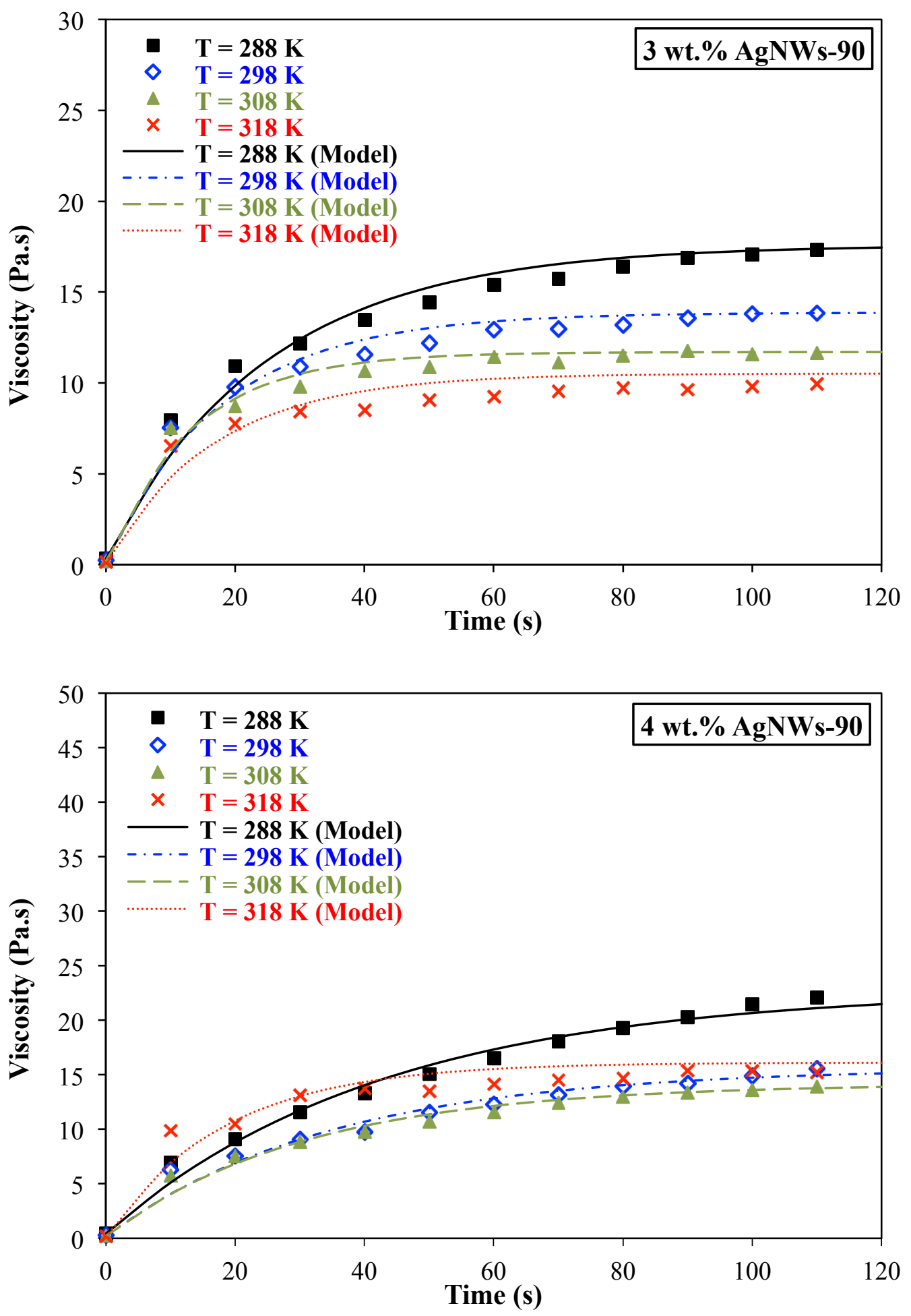

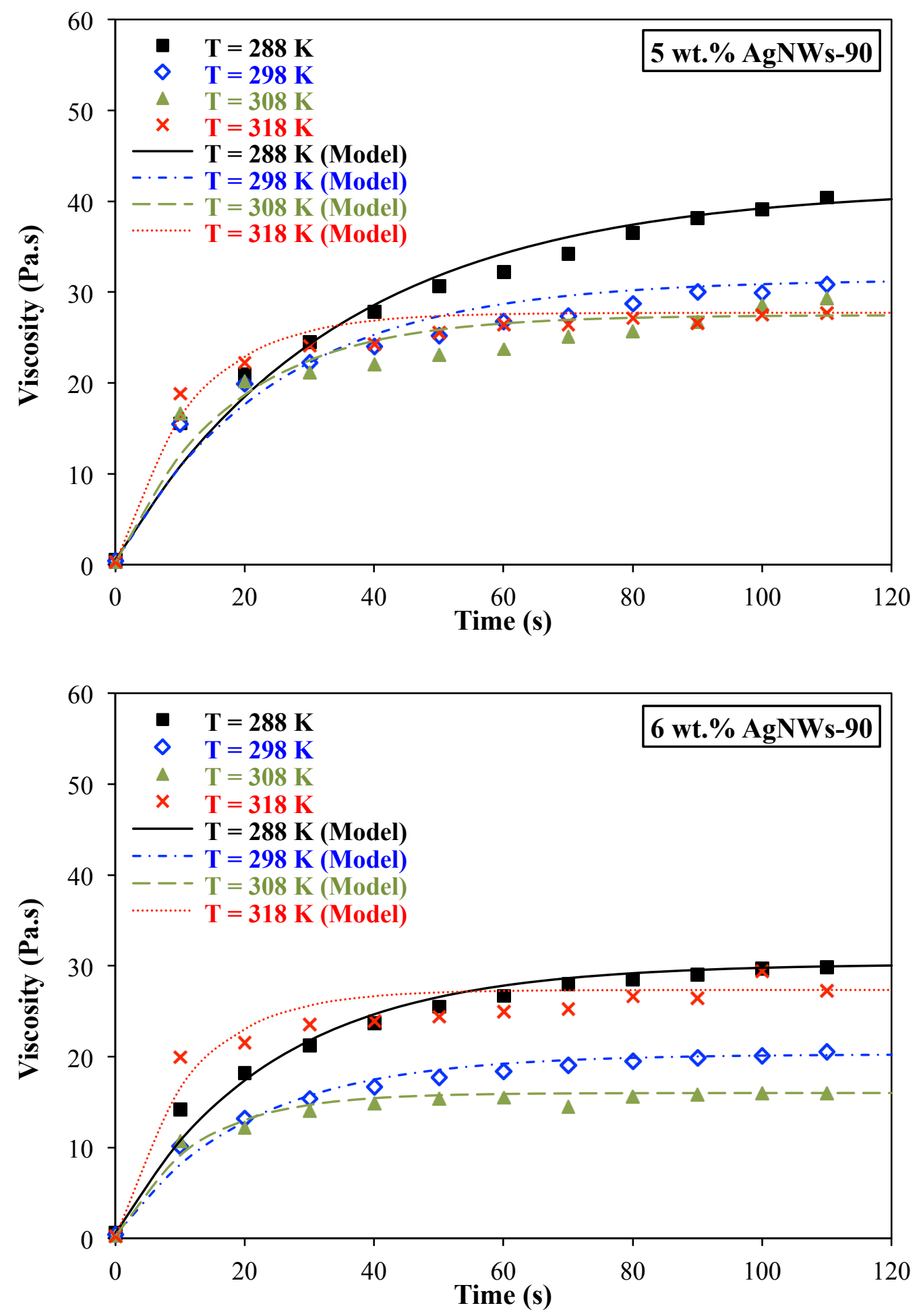


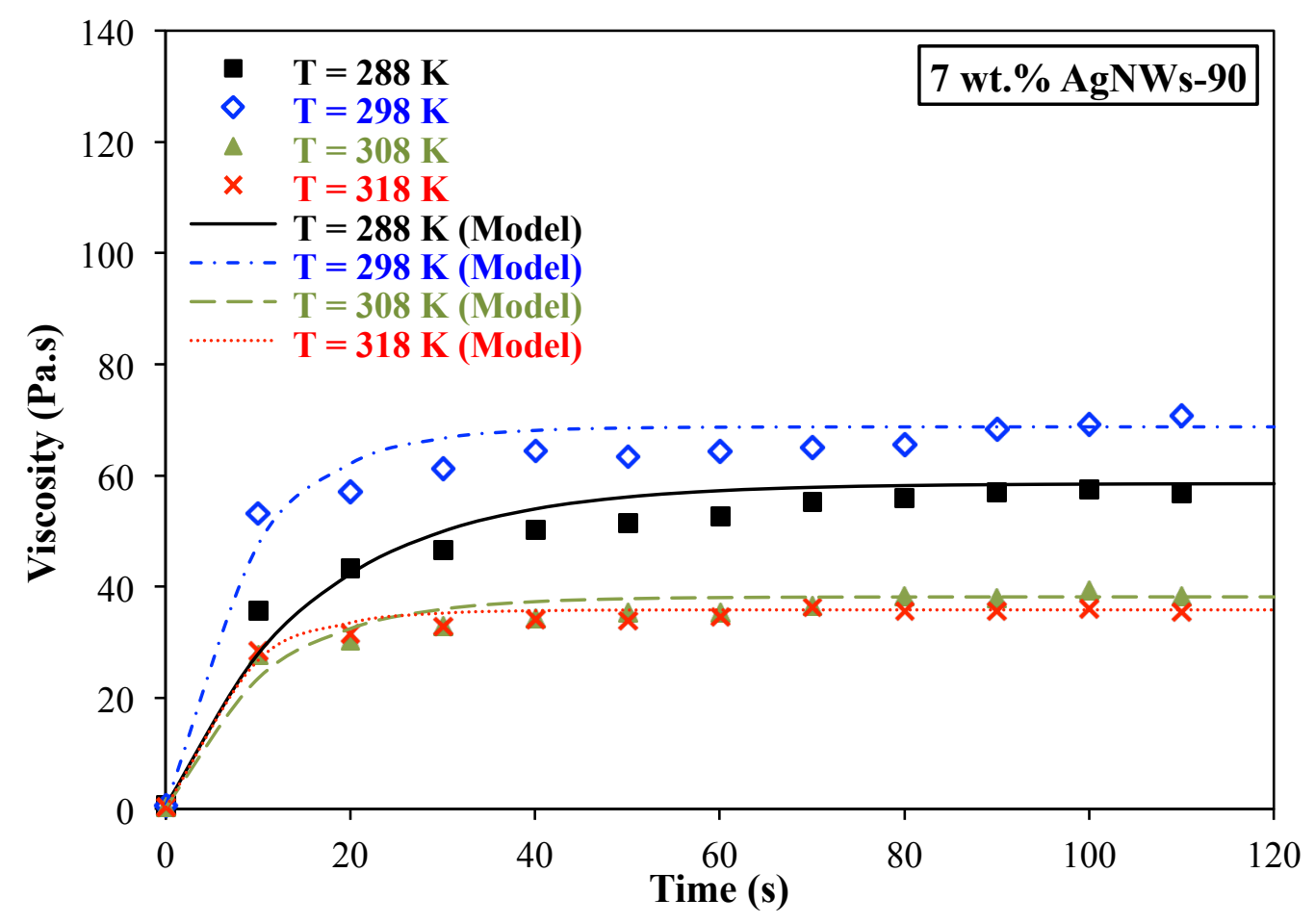

Figure 4. 36: Correlation of the build-up structure for different AgNWs (AgNWs-90) contents at several temperatures.

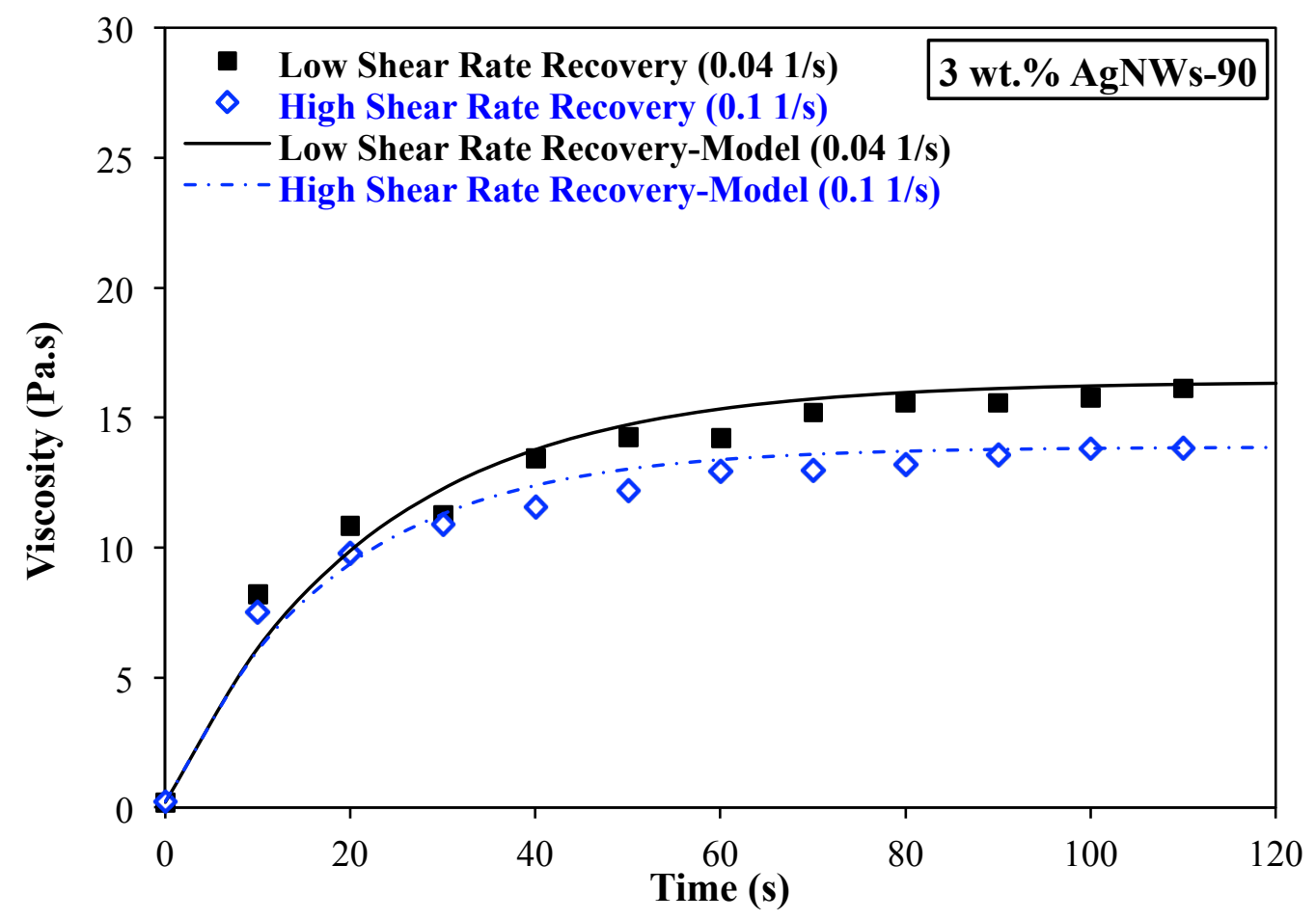



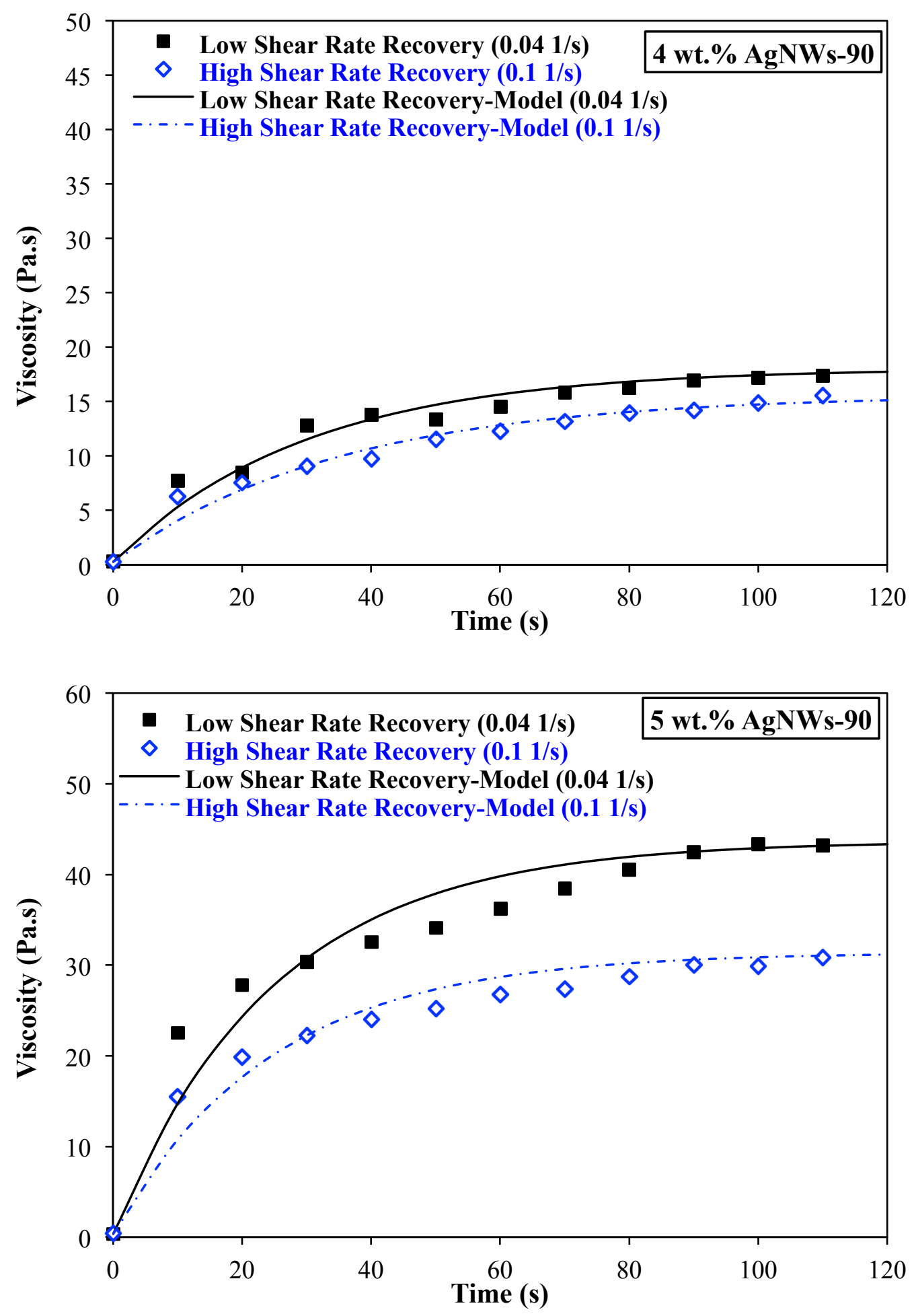


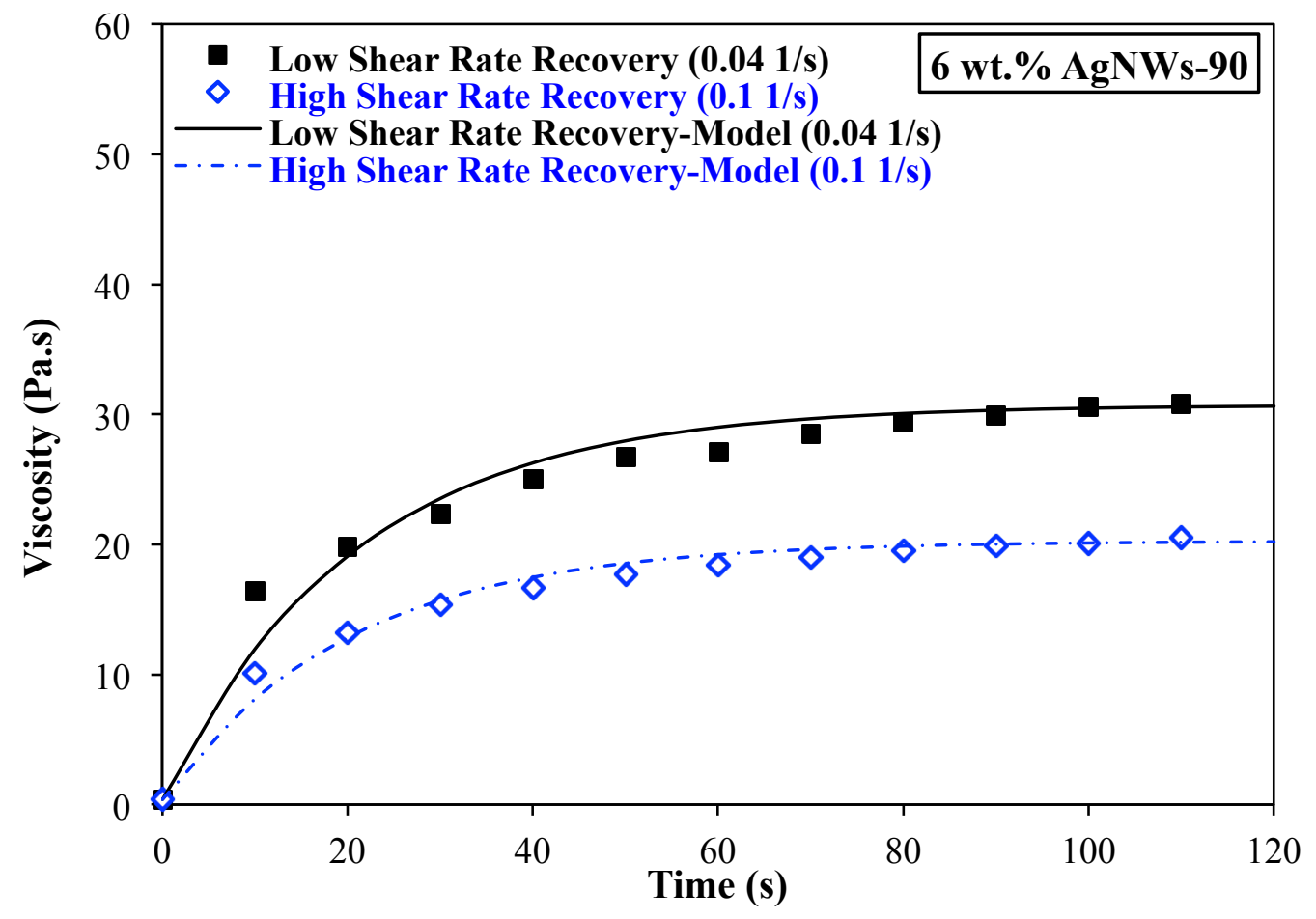

Figure 4. 37: Correlation of the build-up structure for different AgNWs (AgNWs-90) contents at two recovery shear rates.

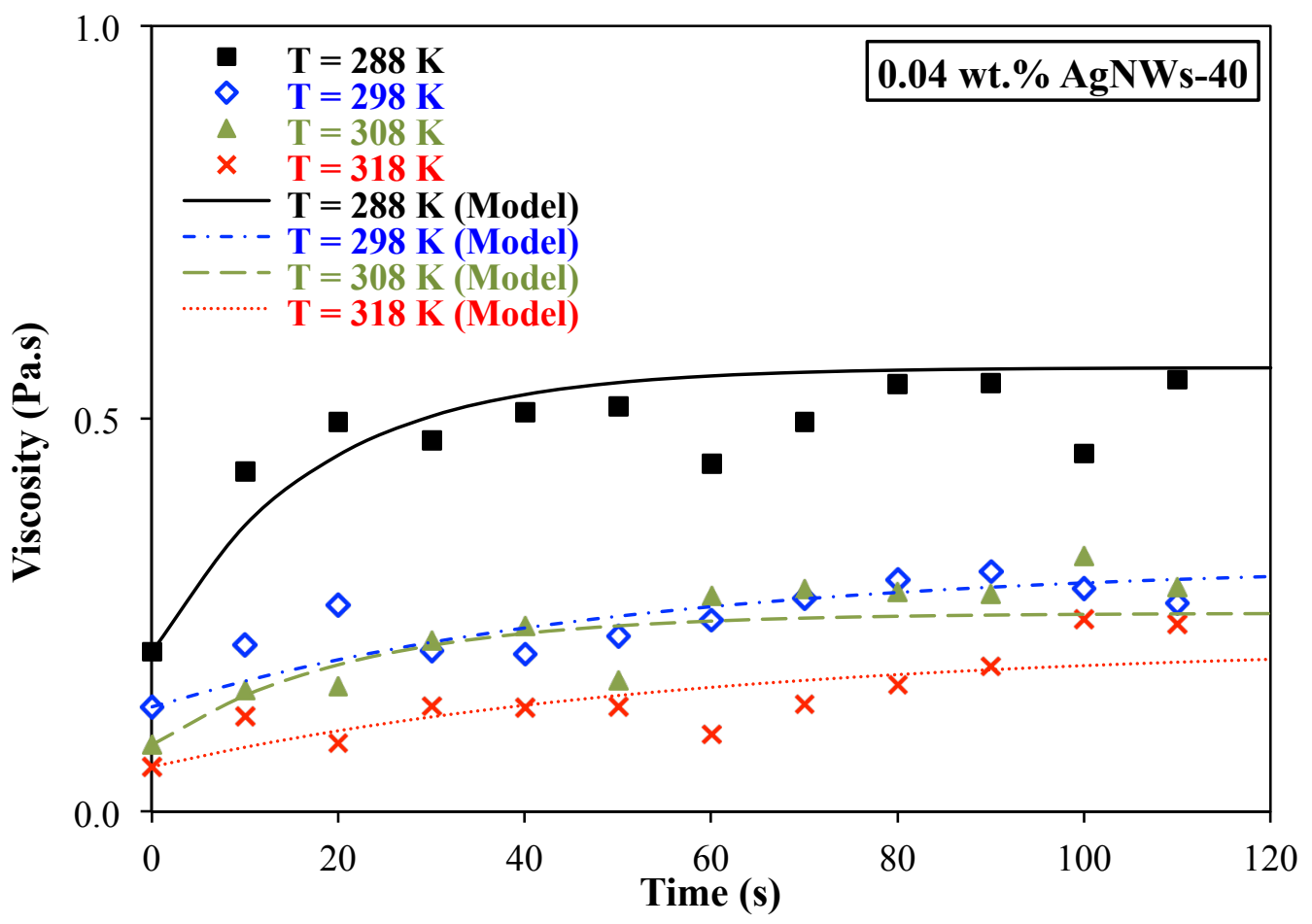



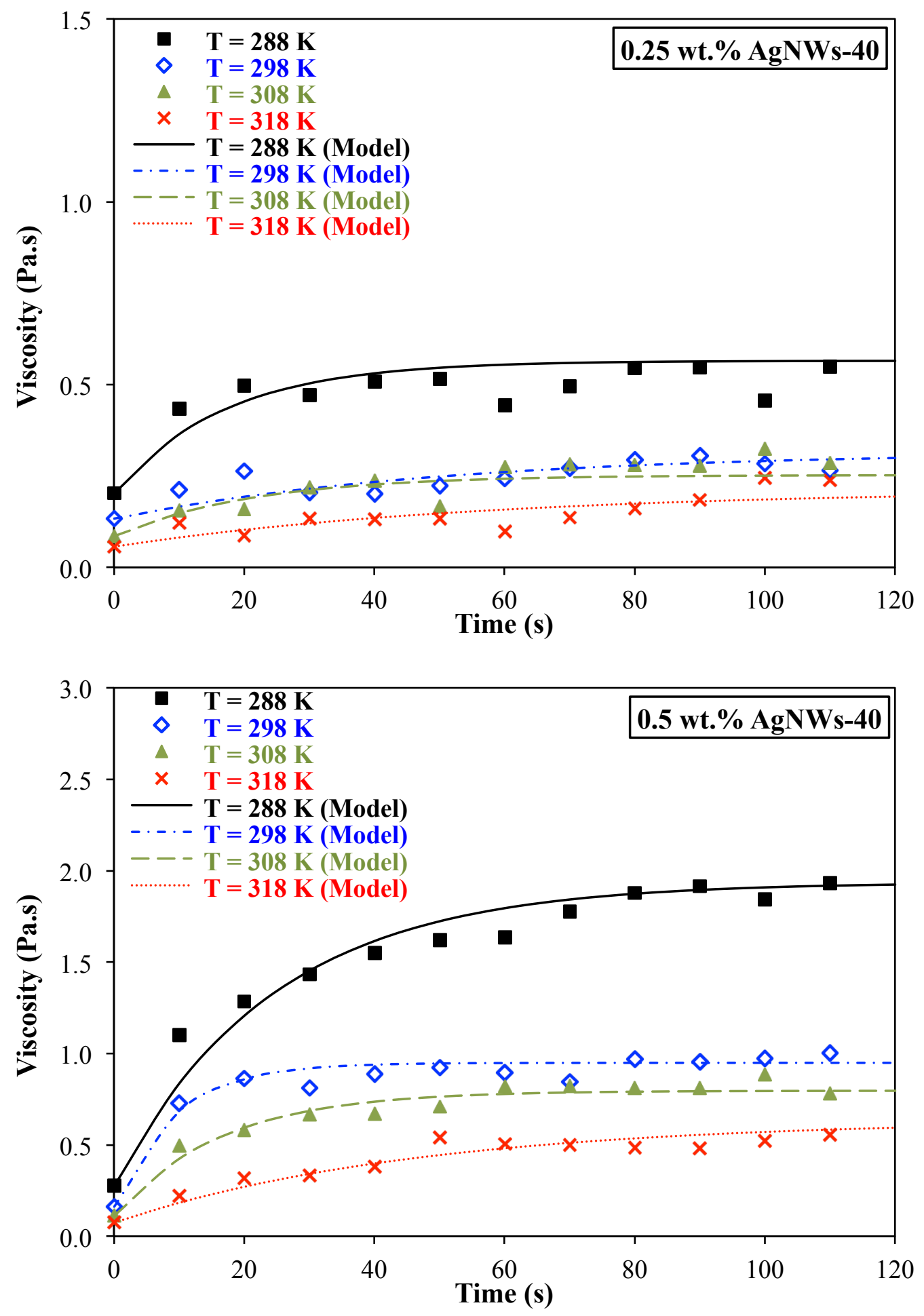

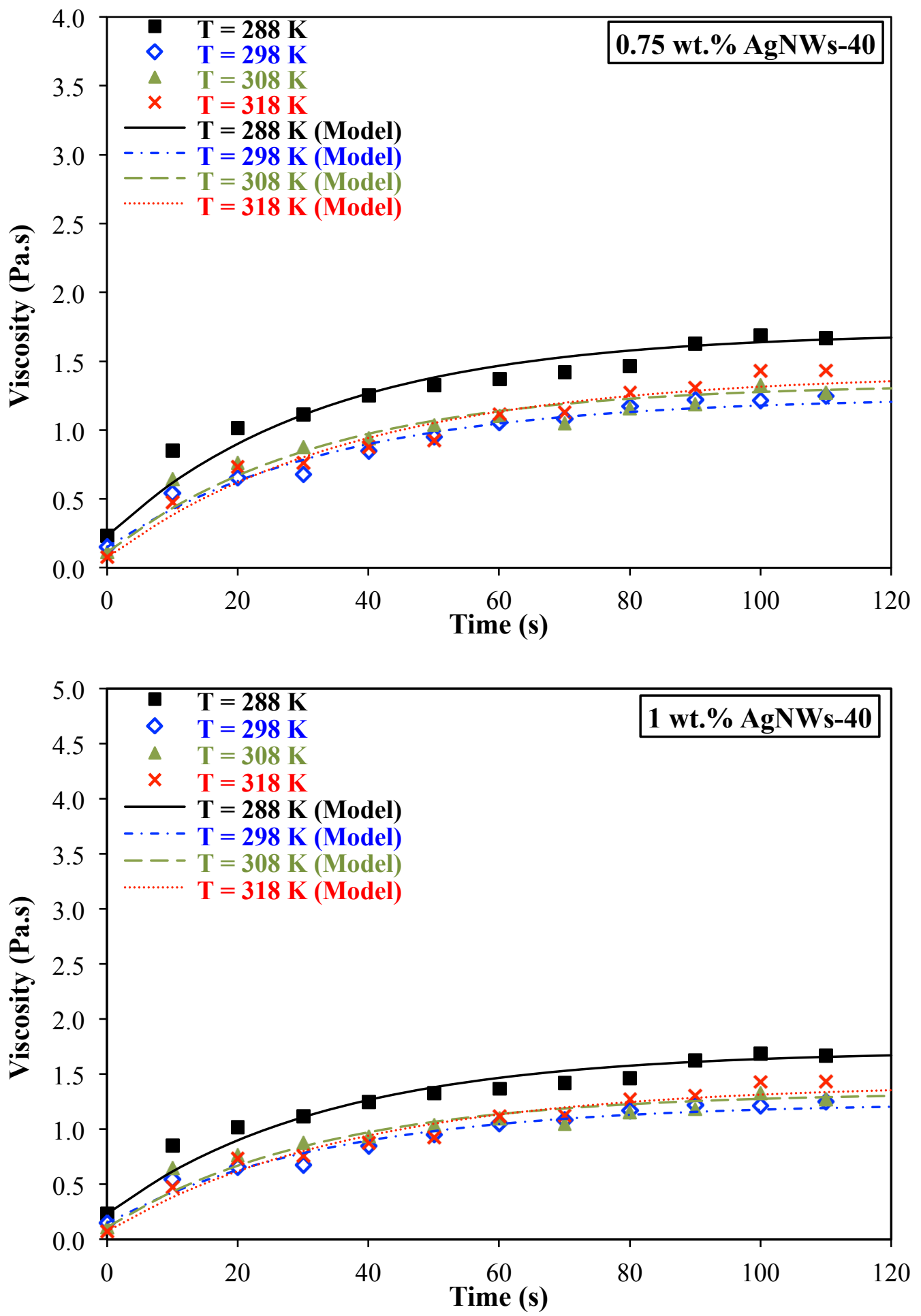

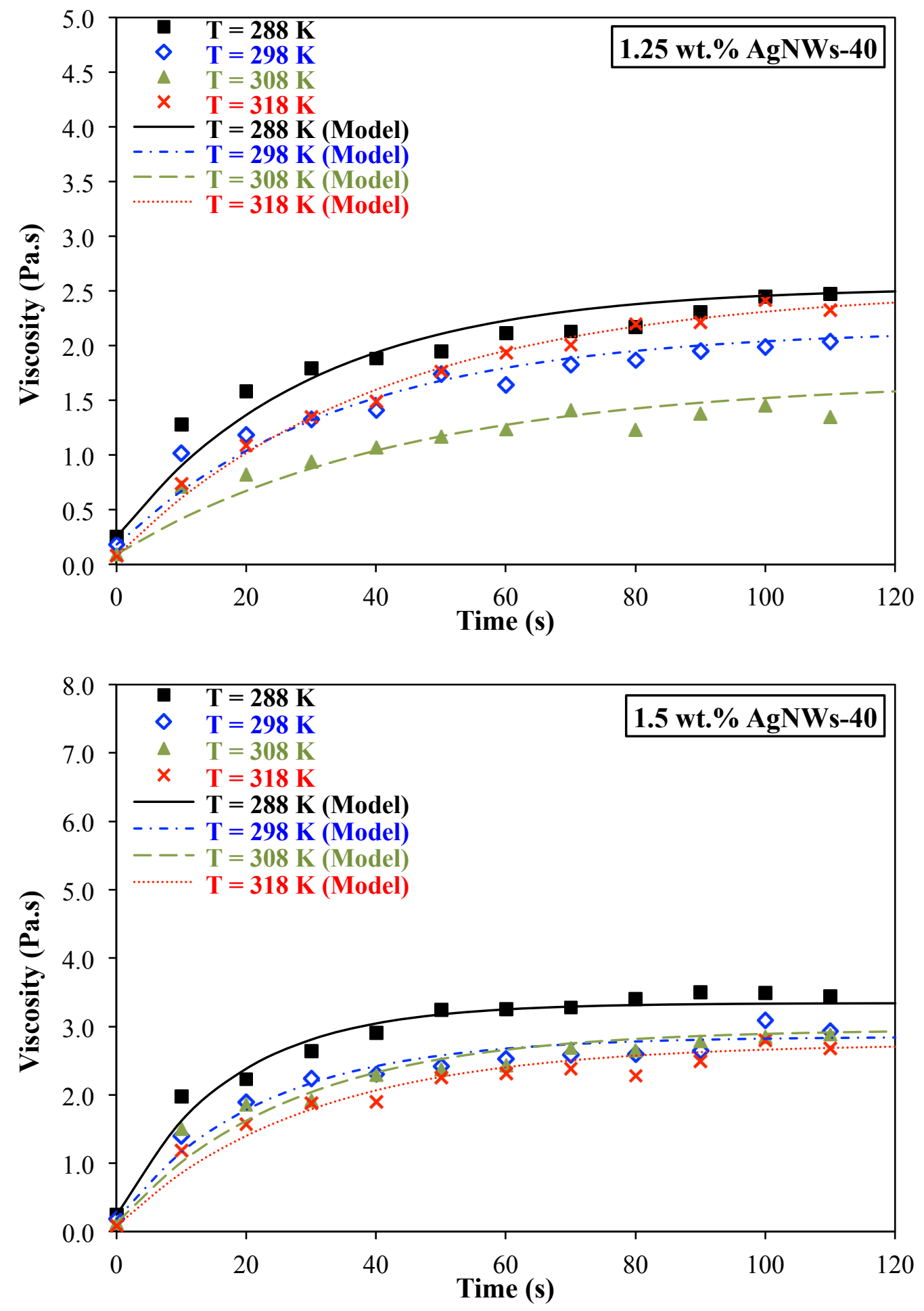

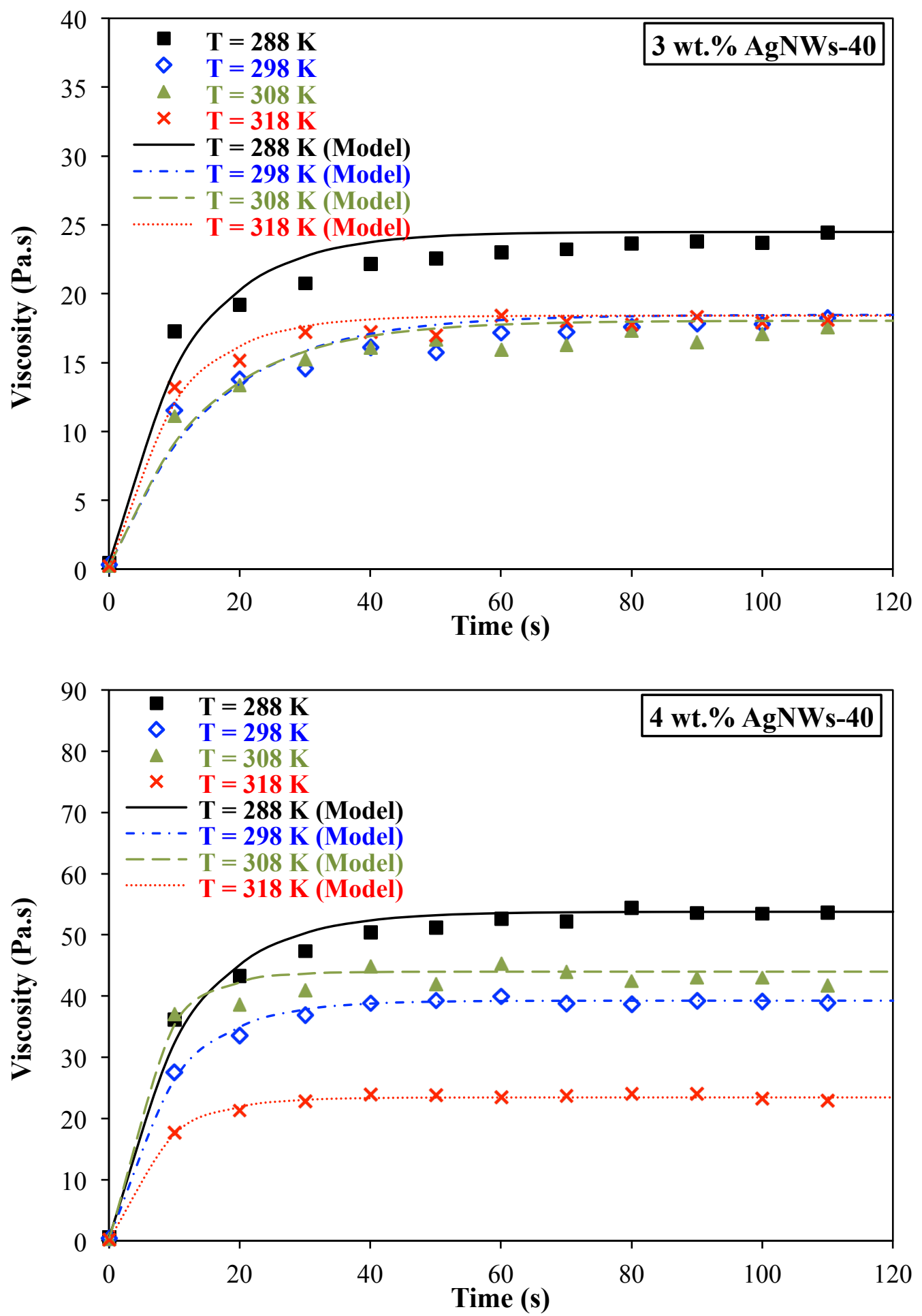

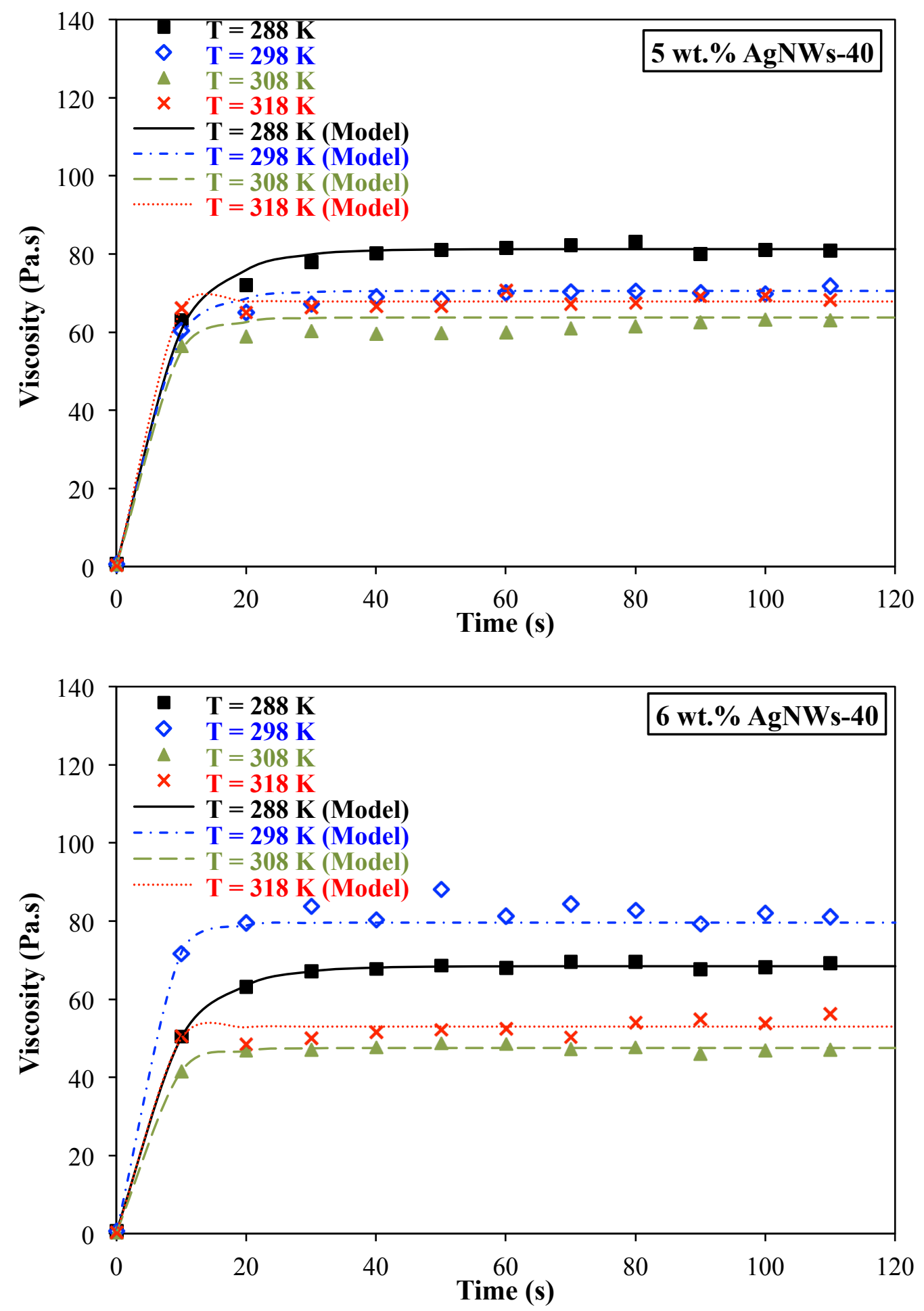

Figure 4. 38: Correlation of the build-up structure for different AgNWs (AgNWs-40) contents at several temperatures. 
A plot of the natural $\log$ of the recovery process rate constant versus the reciprocal of temperature is shown in Figure 4. 39 for AgNWs-90. A linear fit to the data is also shown. Similar results for AgNWs-40 are shown in Figure 4. 40. In every case for the silver nanowire contents of 4 wt.\% and higher, the slope of each linear fit, equal to $-\frac{E_{a}}{R}$, is negative, which gives a positive activation energy. A higher activation energy represents a higher barrier to nanowire movement. The positive intercept of each linear fit is the natural $\log$ of $\frac{1}{\tau_{0}}$, equivalent to a kinetic frequency factor. The frequency factors and activation energies for experiment for the silver nanowire contents of 4 wt.\% and higher are summarized in Table 4. 6.

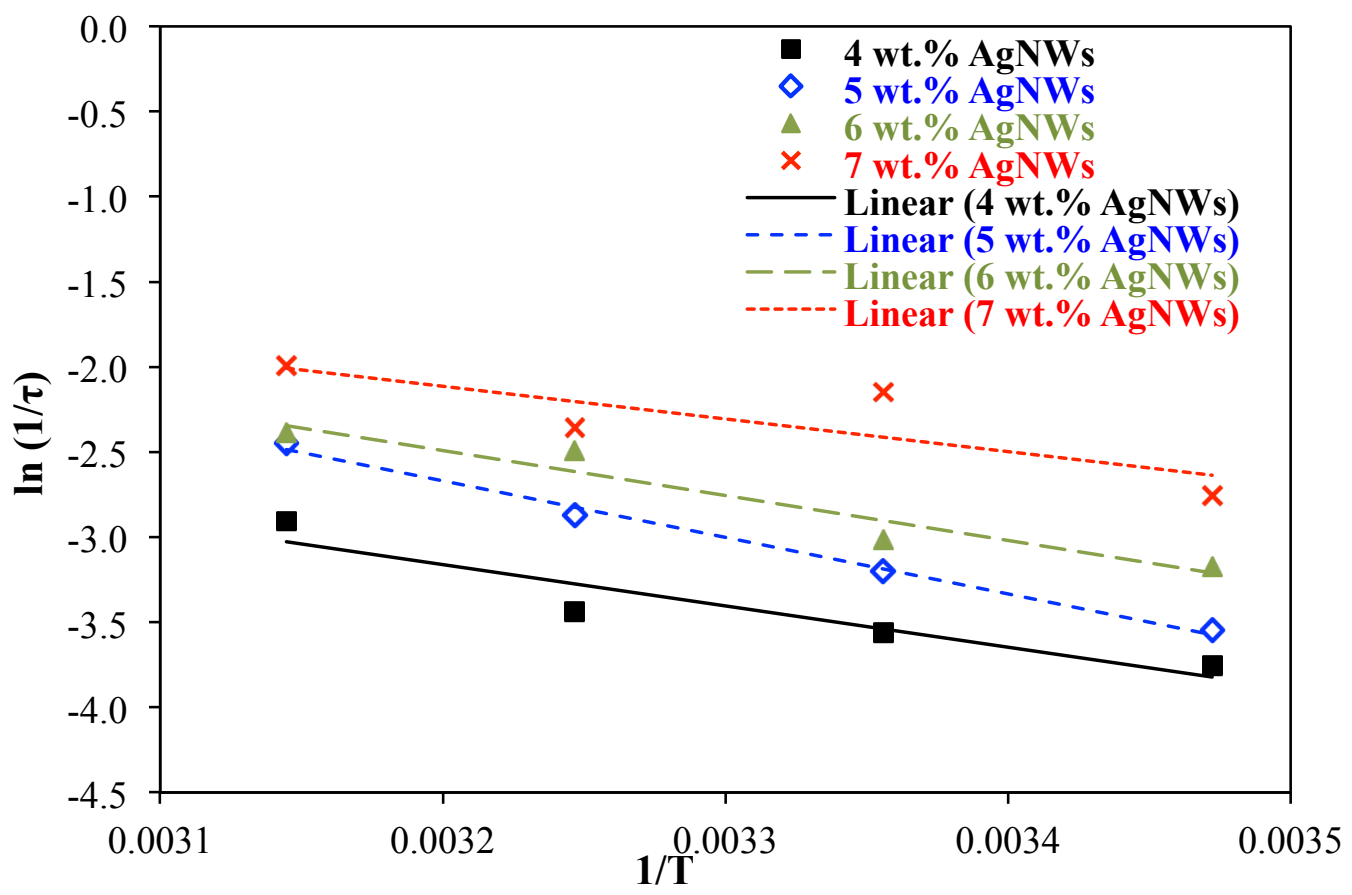

Figure 4. 39: Temperature dependence of the characteristic time for several silver nanowire (AgNWs-90) contents. 

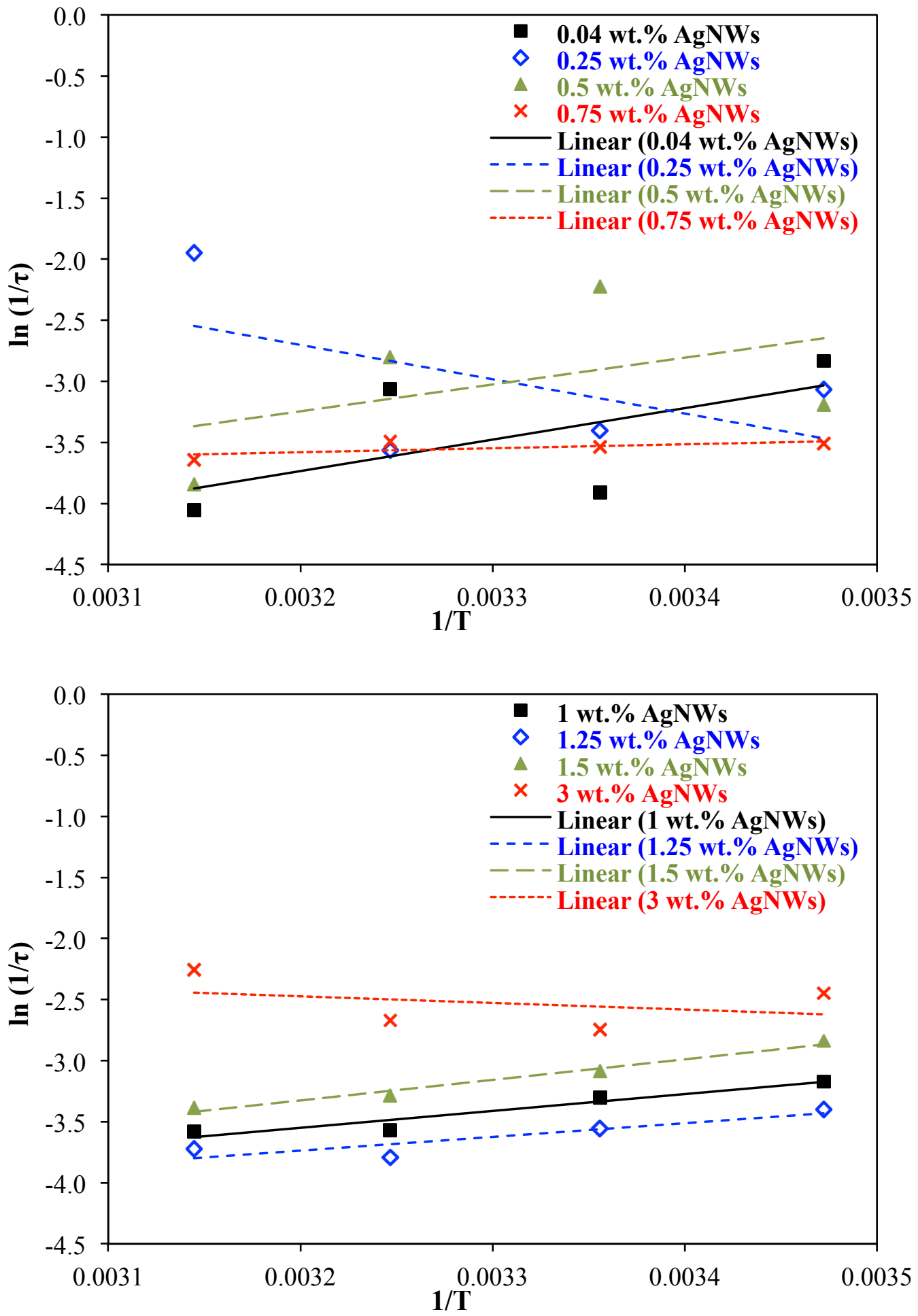


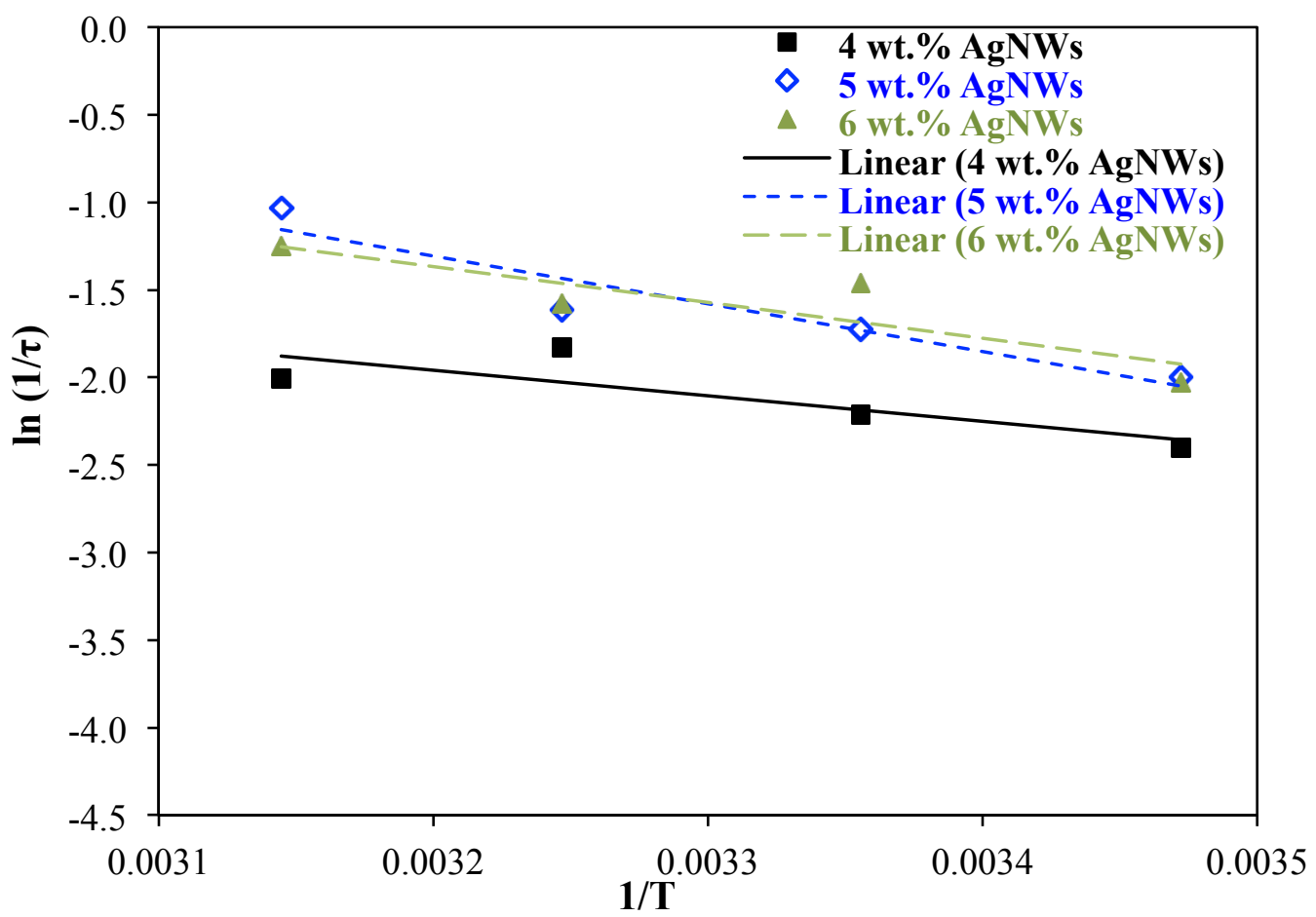

Figure 4. 40: Temperature dependence of the characteristic time for several silver nanowire (AgNWs-40) contents.

Table 4. 6: Activation energy and frequency factor for different silver nanowire contents.

\begin{tabular}{|l|c|c|}
\hline \multicolumn{1}{|c|}{ Sample } & $\begin{array}{c}\text { Frequency Factor } \\
\mathbf{1} / \boldsymbol{\tau}_{\mathbf{0}}\end{array}$ & Activation Energy (kJ) \\
\hline 4 wt.\% AgNW-90 & $9.97 \mathrm{E}+01$ & 20.2 \\
\hline 5 wt.\% AgNW-90 & $2.86 \mathrm{E}+03$ & 27.6 \\
\hline 6 wt.\% AgNW-90 & $3.87 \mathrm{E}+02$ & 22.0 \\
\hline 7 wt.\% AgNW-90 & $5.61 \mathrm{E}+01$ & 16.0 \\
\hline 4 wt.\% AgNW-40 & $1.50 \mathrm{E}+01$ & 12.1 \\
\hline 5 wt.\% AgNW-40 & $1.67 \mathrm{E}+03$ & 22.7 \\
\hline 6 wt.\% AgNW-40 & $1.77 \mathrm{E}+02$ & 17.0 \\
\hline
\end{tabular}




\subsubsection{Dilute/ Semi Dilute/ Concentrated Regimes and Transitions}

The mechanism of viscosity recovery or build-up depends on the suspension concentration because the concentration affects the frequency and character of interactions between wires. The concentration can be categorized as dilute, semi dilute, or concentrated. The criteria for transition from dilute to semi dilute regimes is determined by a dimensionless constant $\beta$, which is defined as $v L^{3}$. The number density of particles and the value of $\beta$ for selected silver nanowire contents are shown in Table 4. 7. This table also provides the values of $\frac{1}{\mathrm{dL}^{2}}$ and $\frac{4.2}{\mathrm{dL}^{2}}$ for both AgNWs-90 and AgNWs-40. These latter parameters are used to distinguish among high concentration regimes including nematic.

The results of $\beta$ values for nanowire contents from 4 to $7 \mathrm{wt} . \%$ show that in all suspensions of AgNWs-90, the AgNWs are not able to diffuse freely based on Brownian motion without any interaction with neighboring wires $(\beta>30)$. Moreover, the values of the number density for different silver nanowire contents are comparable to or higher than the values of $\frac{1}{\mathrm{dL}^{2}}$, which delimits the transition to the concentrated regime. For the highest concentration of silver nanowire $(7 \mathrm{wt} . \%)$, the number density is greater than $\frac{4.2}{\mathrm{dL}^{2}}$, indicating transition to the higher concentrated regime, and obviously the molecular dynamic of the AgNWs cannot be Brownian. All of these results illustrate the strong dependence of the build-up structure mechanism on the AgNWs dimensions (length and diameter of the AgNWs), and AgNWs content [40]. For AgNWs-40 the 0.04 wt. $\%$ is in dilute regime $(\beta<30), 0.25,0.5$, and $0.75 \mathrm{wt} . \%$ are in semi dilute regime $(\beta>30), 1,1.25,1.5$, and $3 \mathrm{wt} . \%$ are in concentrated regime $(\beta>30$ and $v>$ $\frac{1}{\mathrm{dL}^{2}}$, and in all content of the AgNWs of 4 wt.\% or higher, the suspension is in the nematic regime with the criteria of $v>\frac{4.2}{\mathrm{dL}^{2}}$. 
Table 4. 7: Dilute/ semi dilute/ concentrated regime transition criteria for AgNWs-90 (d=90nm, $\mathrm{L}=30 \mu \mathrm{m})$, and AgNWs-40 (d=40nm, $\mathrm{L}=30 \mu \mathrm{m})$.

\begin{tabular}{|c|c|c|c|c|}
\hline Sample & $\frac{1}{d L^{2}}$ & $\frac{4.2}{\mathrm{dL}^{2}}$ & $\beta$ (Dimensionless Constant) & $v$ (Number Density) \\
\hline 4 wt. $\%$ AgNW-90 & \multirow{4}{*}{$1.23 \mathrm{E}+16$} & \multirow{4}{*}{$5.18 \mathrm{E}+16$} & $7.44 \mathrm{E}+02$ & $2.76 \mathrm{E}+16$ \\
\hline 5 wt. $\%$ AgNW- 90 & & & $9.94 \mathrm{E}+02$ & $3.68 \mathrm{E}+16$ \\
\hline 6 wt. $\%$ AgNW-90 & & & $1.27 \mathrm{E}+03$ & $4.70 \mathrm{E}+16$ \\
\hline 7 wt. $\%$ AgNW-90 & & & $1.57 \mathrm{E}+03$ & $5.82 \mathrm{E}+16$ \\
\hline 0.04 wt. $\%$ AgNW-40 & \multirow{11}{*}{$2.78 \mathrm{E}+16$} & \multirow{11}{*}{$1.17 \mathrm{E}+17$} & $2.74 \mathrm{E}+01$ & $1.01 \mathrm{E}+15$ \\
\hline 0.25 wt. $\%$ AgNW-40 & & & $1.75 \mathrm{E}+02$ & $6.47 \mathrm{E}+15$ \\
\hline 0.5 wt. $\%$ AgNW-40 & & & $3.57 \mathrm{E}+02$ & $1.32 \mathrm{E}+16$ \\
\hline 0.75 wt. $\%$ AgNW-40 & & & $5.48 \mathrm{E}+02$ & $2.03 \mathrm{E}+16$ \\
\hline 1 wt. $\%$ AgNW-40 & & & $7.47 \mathrm{E}+02$ & $2.77 \mathrm{E}+16$ \\
\hline 1.25 wt. $\%$ AgNW-40 & & & $9.54 \mathrm{E}+02$ & $3.53 \mathrm{E}+16$ \\
\hline 1.5 wt. $\%$ AgNW-40 & & & $1.17 \mathrm{E}+03$ & $4.33 \mathrm{E}+16$ \\
\hline 3 wt. $\%$ AgNW-40 & & & $2.63 \mathrm{E}+03$ & $9.74 \mathrm{E}+16$ \\
\hline 4 wt.\% AgNW-40 & & & $3.77 \mathrm{E}+03$ & $1.39 \mathrm{E}+17$ \\
\hline 5 wt. $\%$ AgNW-40 & & & $5.03 \mathrm{E}+03$ & $1.86 \mathrm{E}+17$ \\
\hline 6 wt. $\%$ AgNW-40 & & & $6.43 \mathrm{E}+03$ & $2.38 \mathrm{E}+17$ \\
\hline
\end{tabular}

\subsubsection{Build-up Mechanism}

The values of characteristic times and the Peclet number as function of concentration are summarized at several temperatures in Table 4. 8. Similar results at different recovery shear rates are summarized in Table 4. 9 for AgNWs-90. The values of characteristic times and the Peclet numbers for different AgNWs (AgNWs-40) contents are summarized at several temperatures in

Table 4. 10. The Peclet numbers indicate that Brownian motion is negligible in these experiments. Therefore, the rearrangement and recovery process are mainly due to hydrodynamic forces and wire-wire interactions. The very large Peclet numbers indicate that the time required for a wire to rotate is large in comparison to the $120 \mathrm{~s}$ duration of the experimental recovery time in the third step of the $\mathrm{PH}$ test [39]. Hence, the recovery occurs through thermodynamic and hydrodynamic interactions such as wire-wire forces, wire-fluid forces, and viscous forces rather than Brownian motion. At constant temperature, the Peclet number increases with increasing the AgNWs content, and therefor wire-wire interactions increase with 
increasing concentration of AgNWs. Moreover, the Peclet number is lower at lower recovery shear rate and higher temperature.

Table 4. 8: Peclet number and characteristic time of selected silver nanowire (AgNWs-90) contents at different temperatures.

\begin{tabular}{|c|c|c|c|}
\hline Sample & Temperature (K) & Characteristic Time (s) & Peclet Number \\
\hline \multirow{4}{*}{ 4 wt.\% AgNW-90 } & 288 & 42.8 & $1.39 \mathrm{E}+13$ \\
\cline { 2 - 4 } & 298 & 35.3 & $9.22 \mathrm{E}+12$ \\
\cline { 2 - 4 } & 308 & 31.2 & $8.09 \mathrm{E}+12$ \\
\hline \multirow{5}{*}{ 5 wt.\% AgNW-90 } & 318 & 18.3 & $8.90 \mathrm{E}+12$ \\
\cline { 2 - 4 } & 288 & 34.7 & $4.52 \mathrm{E}+13$ \\
\cline { 2 - 4 } & 298 & 24.6 & $3.30 \mathrm{E}+13$ \\
\hline \multirow{4}{*}{ 6 wt.\% AgNW-90 } & 308 & 17.7 & $2.80 \mathrm{E}+13$ \\
\cline { 2 - 4 } & 288 & 11.5 & $2.73 \mathrm{E}+13$ \\
\cline { 2 - 4 } & 318 & 24.0 & $5.37 \mathrm{E}+13$ \\
\hline \multirow{3}{*}{ 7 wt.\% AgNW-90 } & 308 & 20.4 & $3.48 \mathrm{E}+13$ \\
\cline { 2 - 4 } & 288 & 12.1 & $2.66 \mathrm{E}+13$ \\
\cline { 2 - 4 } & 298 & 10.9 & $1.40 \mathrm{E}+13$ \\
\hline
\end{tabular}

Table 4. 9: Peclet number and characteristic time of selected silver nanowire (AgNWs-90) contents at different viscosity recovery shear rates.

\begin{tabular}{|c|c|c|c|}
\hline Sample & Shear Rate (1/s) & Characteristic Time (s) & Peclet Number \\
\hline 4 wt.\% AgNW-90 & 0.10 & 35.3 & $9.92 \mathrm{E}+12$ \\
\cline { 2 - 4 } & 0.04 & 30.0 & $4.26 \mathrm{E}+12$ \\
\hline 5 wt.\% AgNW-90 & 0.10 & 24.6 & $3.30 \mathrm{E}+13$ \\
\hline \multirow{2}{*}{ 6 wt.\% AgNW-90 } & 0.04 & 24.9 & $1.84 \mathrm{E}+13$ \\
\cline { 2 - 4 } & 0.10 & 20.4 & $3.48 \mathrm{E}+13$ \\
\hline
\end{tabular}


Table 4. 10: Peclet number and characteristic time of selected silver nanowire (AgNWs-40) contents at different temperatures.

\begin{tabular}{|c|c|c|c|}
\hline Sample & Temperature (K) & Characteristic Time (s) & Peclet Number \\
\hline \multirow{4}{*}{0.04 wt. $\%$ AgNW-40 } & 288 & 17.0 & $4.10 \mathrm{E}+08$ \\
\hline & 298 & 49.9 & $2.22 \mathrm{E}+08$ \\
\hline & 308 & 21.4 & $1.72 \mathrm{E}+08$ \\
\hline & 318 & 57.5 & $1.40 \mathrm{E}+08$ \\
\hline \multirow{4}{*}{0.25 wt. $\%$ AgNW-40 } & 288 & 21.5 & $2.43 \mathrm{E}+10$ \\
\hline & 298 & 30.1 & $1.34 \mathrm{E}+10$ \\
\hline & 308 & 35.3 & $1.16 \mathrm{E}+10$ \\
\hline & 318 & 7.01 & $4.71 \mathrm{E}+09$ \\
\hline \multirow{4}{*}{0.5 wt. $\%$ AgNW-40 } & 288 & 24.3 & $2.39 \mathrm{E}+11$ \\
\hline & 298 & 9.24 & $1.13 \mathrm{E}+11$ \\
\hline & 308 & 16.5 & $9.20 \mathrm{E}+10$ \\
\hline & 318 & 46.7 & $7.13 \mathrm{E}+10$ \\
\hline \multirow{4}{*}{0.75 wt. $\%$ AgNW-40 } & 288 & 33.4 & $4.98 \mathrm{E}+11$ \\
\hline & 298 & 34.5 & $3.48 \mathrm{E}+11$ \\
\hline & 308 & 32.9 & $3.63 \mathrm{E}+11$ \\
\hline & 318 & 38.3 & $3.72 \mathrm{E}+11$ \\
\hline \multirow{4}{*}{1 wt. $\%$ AgNW-40 } & 288 & 23.8 & $1.59 \mathrm{E}+12$ \\
\hline & 298 & 27.1 & $1.66 \mathrm{E}+12$ \\
\hline & 308 & 35.5 & $1.31 \mathrm{E}+12$ \\
\hline & 318 & 35.9 & $1.11 \mathrm{E}+12$ \\
\hline \multirow{4}{*}{1.25 wt. $\%$ AgNW-40 } & 288 & 30.0 & $2.23 \mathrm{E}+12$ \\
\hline & 298 & 35.1 & $1.83 \mathrm{E}+12$ \\
\hline & 308 & 44.5 & $1.39 \mathrm{E}+12$ \\
\hline & 318 & 41.4 & $2.02 \mathrm{E}+12$ \\
\hline \multirow{4}{*}{1.5 wt. $\%$ AgNW-40 } & 288 & 17.1 & $4.42 \mathrm{E}+12$ \\
\hline & 298 & 22.0 & $3.64 \mathrm{E}+12$ \\
\hline & 308 & 26.7 & $3.66 \mathrm{E}+12$ \\
\hline & 318 & 29.5 & $3.29 \mathrm{E}+12$ \\
\hline \multirow{4}{*}{3 wt. $\%$ AgNW-40 } & 288 & 11.6 & $1.64 \mathrm{E}+14$ \\
\hline & 298 & 15.5 & $1.19 \mathrm{E}+14$ \\
\hline & 308 & 14.5 & $1.13 \mathrm{E}+14$ \\
\hline & 318 & 9.56 & $1.12 \mathrm{E}+14$ \\
\hline \multirow{4}{*}{4 wt. $\%$ AgNW-40 } & 288 & 11.1 & $7.38 \mathrm{E}+14$ \\
\hline & 298 & 9.12 & $5.20 \mathrm{E}+14$ \\
\hline & 308 & 6.22 & $5.64 \mathrm{E}+14$ \\
\hline & 318 & 7.44 & $2.91 \mathrm{E}+14$ \\
\hline \multirow{4}{*}{5 wt. $\%$ AgNW-40 } & 288 & 7.37 & $1.99 \mathrm{E}+15$ \\
\hline & 298 & 5.61 & $1.67 \mathrm{E}+15$ \\
\hline & 308 & 5.02 & $1.46 \mathrm{E}+15$ \\
\hline & 318 & 2.82 & $1.50 \mathrm{E}+15$ \\
\hline \multirow{4}{*}{6 wt. $\%$ AgNW-40 } & 288 & 7.63 & $2.73 \mathrm{E}+15$ \\
\hline & 298 & 4.30 & $3.07 \mathrm{E}+15$ \\
\hline & 308 & 4.86 & $1.78 \mathrm{E}+15$ \\
\hline & 318 & 3.50 & $1.92 \mathrm{E}+15$ \\
\hline
\end{tabular}


Plots of the natural $\log$ of $\eta_{I}$, and $\eta_{R}$ versus $\frac{1}{T}$ are shown in Figure 4. 41, and Figure 4. 42, respectively for several silver nanowire (AgNWs-90) contents. Figure 4. 43, and Figure 4. 44, show similar data for AgNWs-40. The plots of natural log of the initial and recovered viscosity versus $\frac{1}{\mathrm{~T}}$ have positive slopes, which corresponds to a positive activation enthalpy for flow. It has been suggested that when there are different simultaneous mechanisms, and the nature of the process is not clear, the behavior can be quantified in term of activation energy by evaluation of the sign of the activation enthalpy and entropy. The values of the activation enthalpy, and activation entropy based on these diagrams for different contents of the silver nanowire of the AgNWs-90 and AgNWs-40 are shown in Table 4. 11, and Table 4. 12, respectively.

The shear thinning behavior of the inks arises from the oriented arrangements of wires under shear. In this situation, there should be a negative change of activation entropy in the shift from high shear to low shear, which tends to increase viscosity at more random arrangements of wires. This is compensated by a decrease in activation enthalpy, as there is lower activation enthalpy for the low shear rate compared to the high shear rate. These are opposing tendencies that the activation enthalpy and activation entropy have on viscosity [41]. Although the exact structure of systems during recovery process is unclear, the recovery structure is driven by thermodynamics, and the reforming of the nanowire orientation from more orientated to more random structure strongly depends on thermodynamic of the system $[38,60]$. The transition from high shear to low shear during the recovery process tends that the wire-wire interactional factors dominate the structural ones [61]. Comparison between the $\Delta \mathrm{H}_{\mathrm{I}}$ and $\Delta \mathrm{H}_{\mathrm{R}}$ indicates the interfacial enthalpy lost during the recovery process, which causes the negative activation enthalpy $(\Delta \mathrm{H})$, and definitely the enthalpy dominancy is considerable in this process [62]. Due to the lack of information regarding the $\mathrm{K}^{\prime}$ values, which is the pre-exponential constant in the relation of temperature 
dependence of viscosity, it is impossible to report the absolute values of the activation entropy $(\Delta \mathrm{S})$. So it is hard to predict the degree of the entropy dominancy during the recovery process.

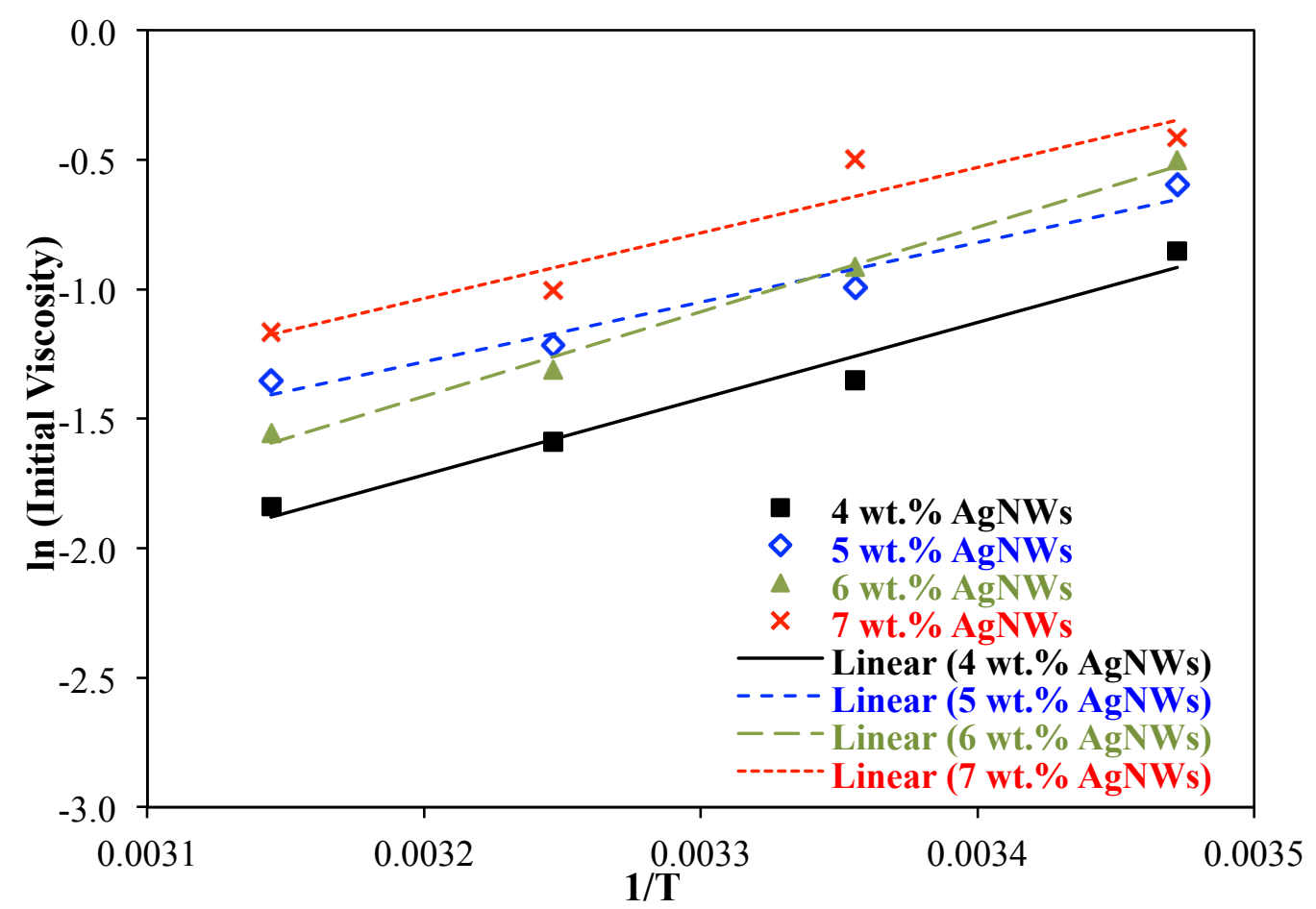

Figure 4. 41: Temperature dependence of the initial viscosity for different silver nanowire (AgNWs-90) contents. 


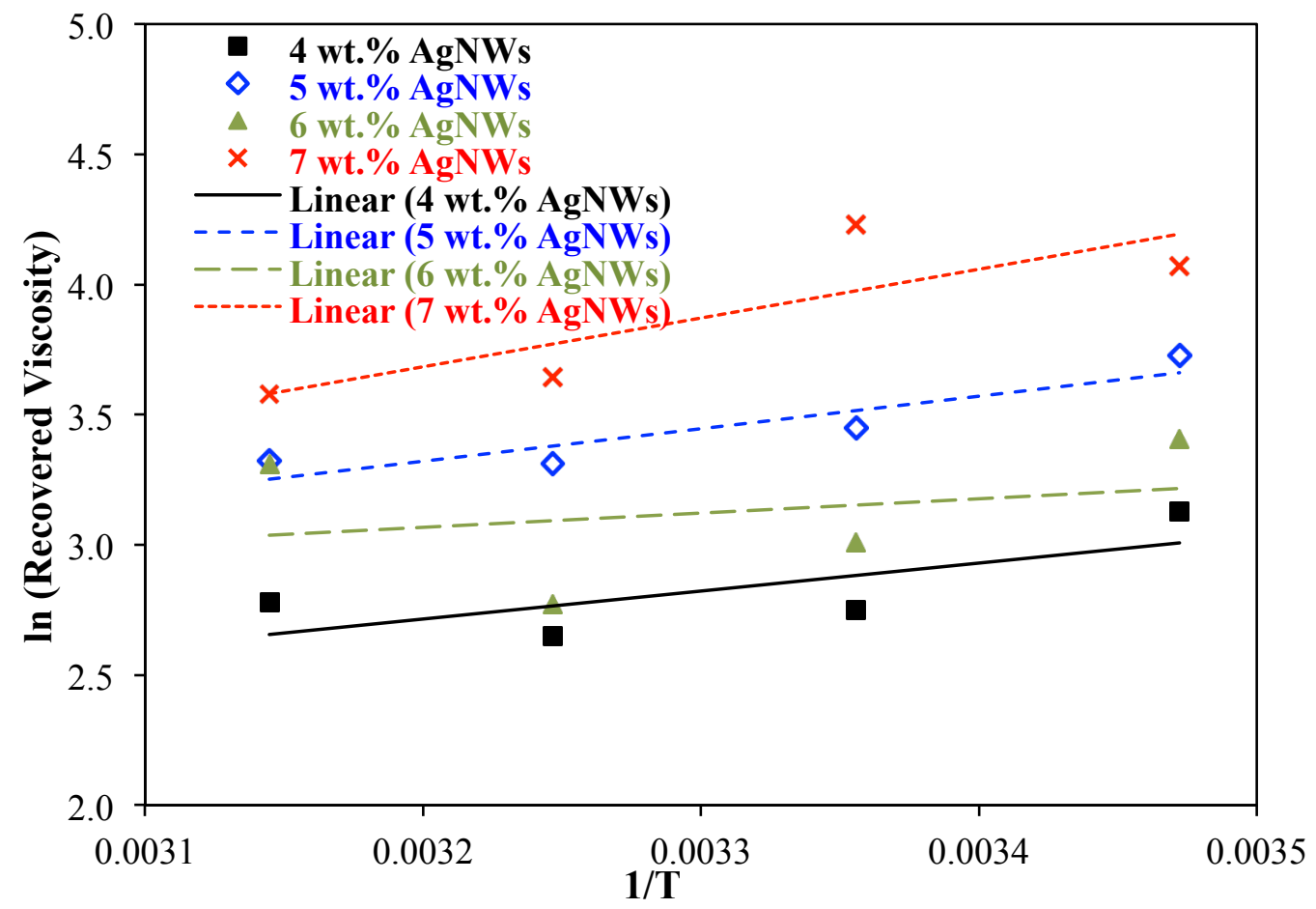

Figure 4. 42: Temperature dependence of the recovered viscosity for different silver nanowire (AgNWs-90) contents. 

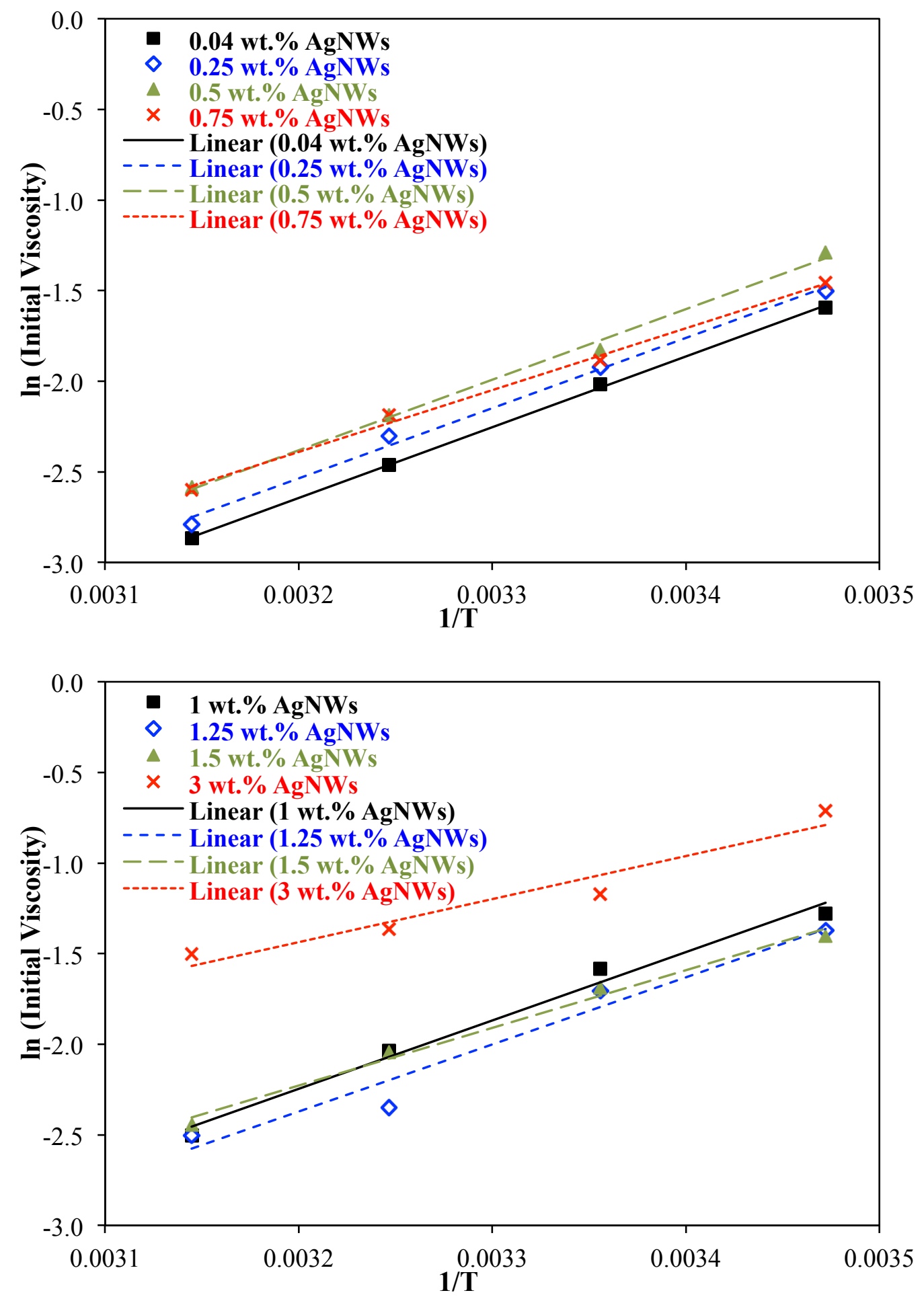


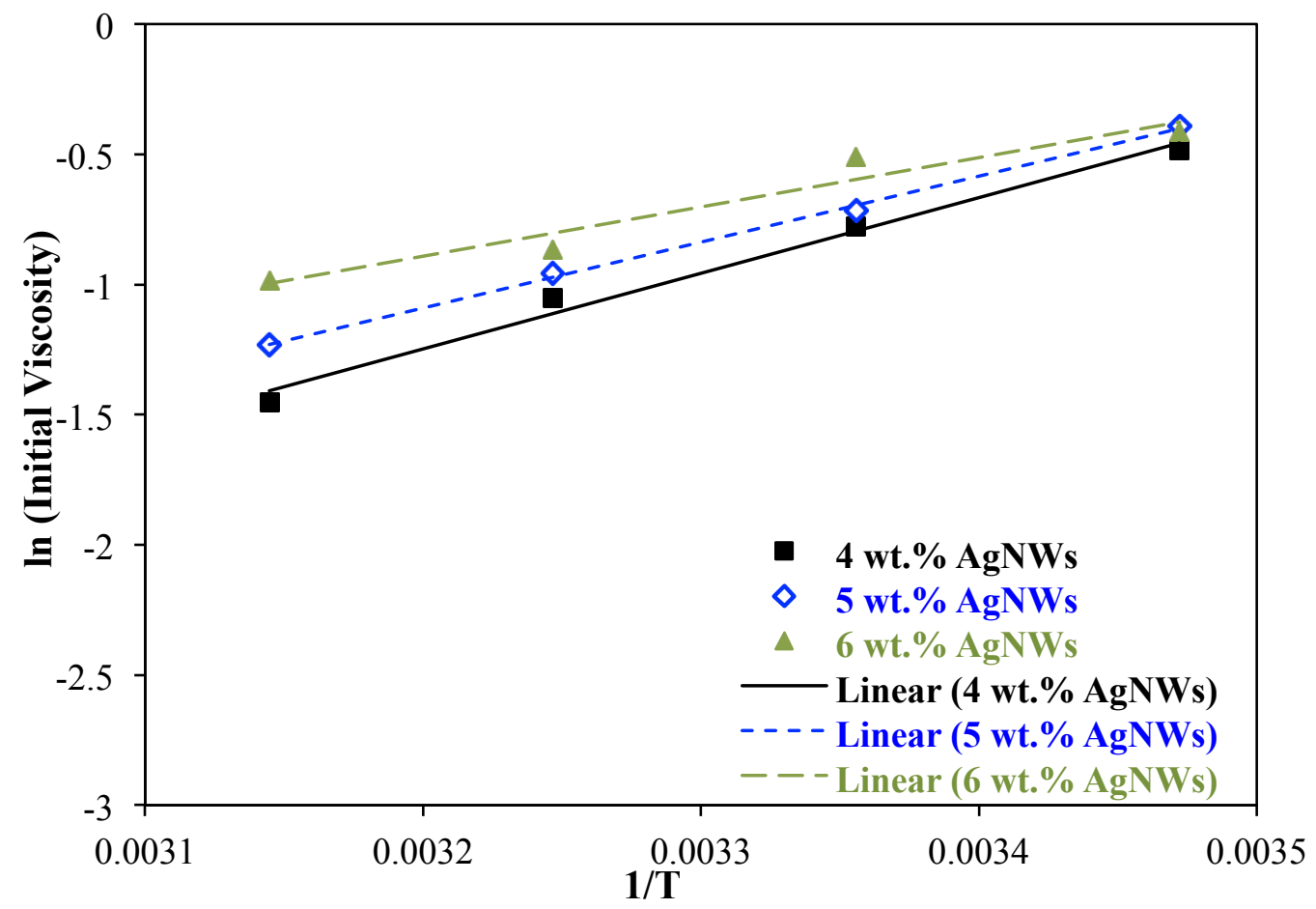

Figure 4. 43: Temperature dependence of the initial viscosity for different silver nanowire (AgNWs-40) contents.

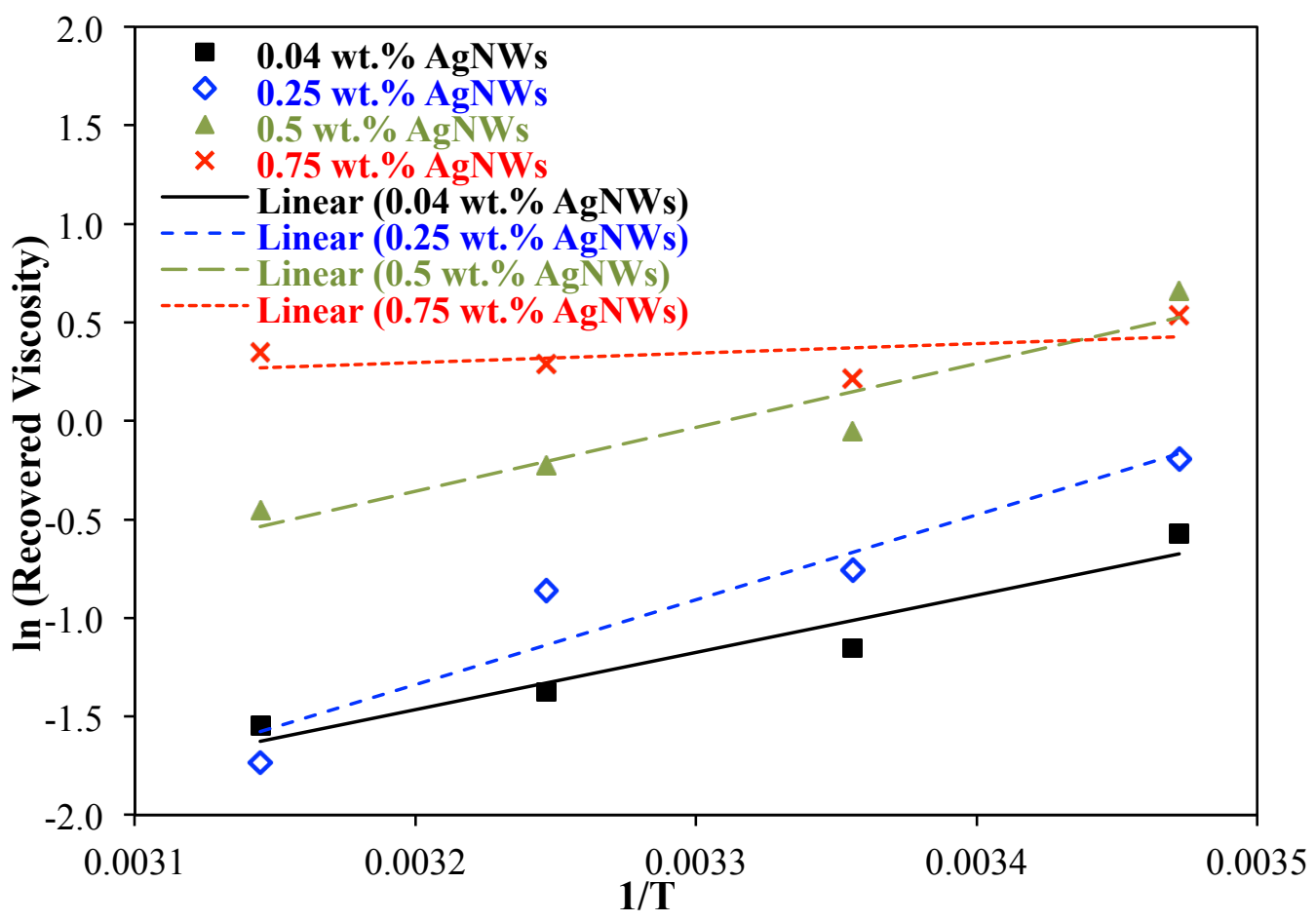



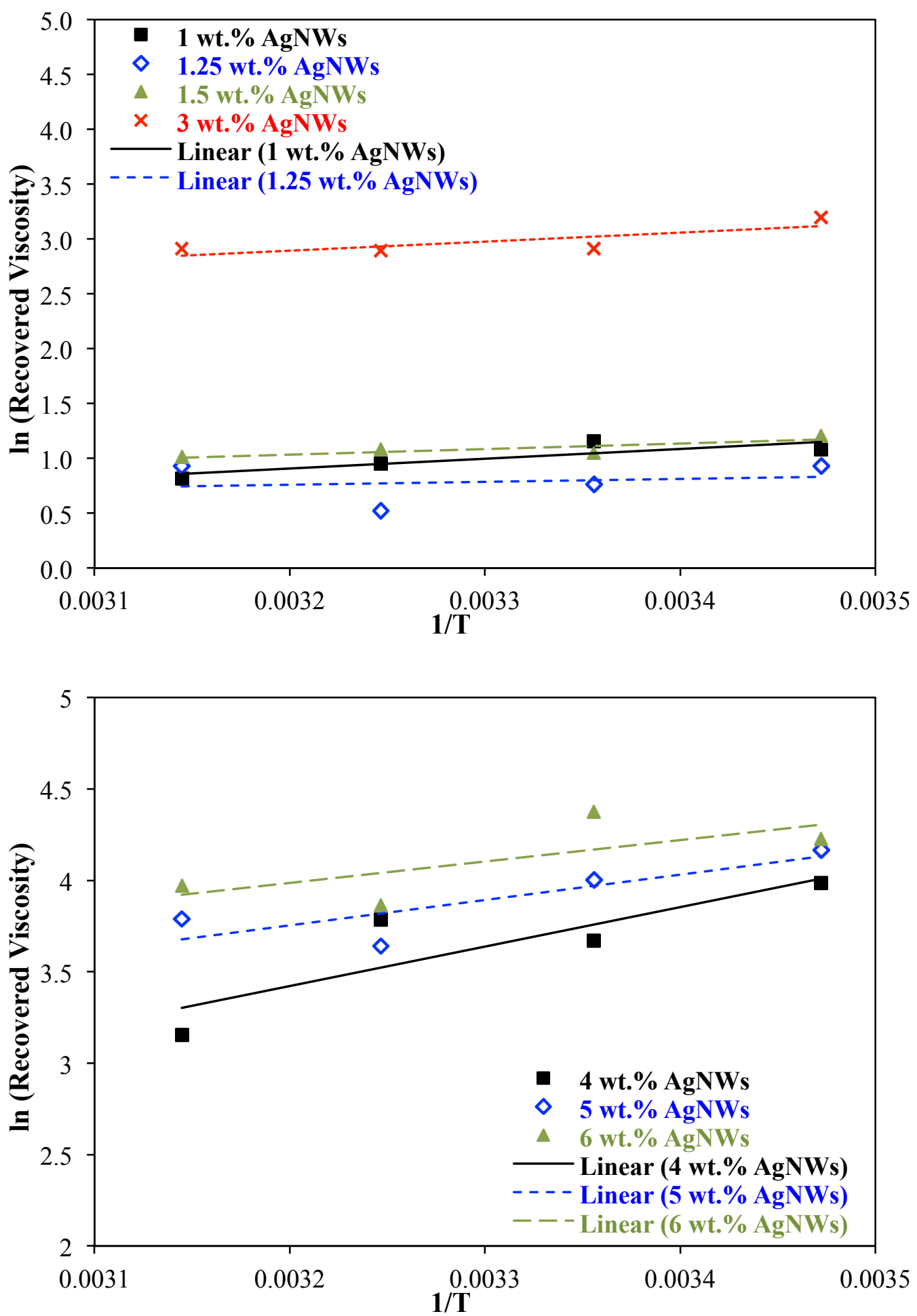

Figure 4. 44: Temperature dependence of the recovered viscosity for different silver nanowire (AgNWs-40) contents. 
Table 4. 11: Activation enthalpy, and activation entropy during recovery process for different silver nanowire (AgNWs-90) contents.

\begin{tabular}{|c|c|c|c|c|c|}
\hline Sample & $\boldsymbol{\Delta H}_{\mathbf{I}}(\mathbf{K J})$ & $\boldsymbol{\Delta} \mathbf{H}_{\mathbf{R}}(\mathbf{K J})$ & $\boldsymbol{\Delta} \mathbf{H}(\mathbf{K J})$ & $\ln \left(\mathbf{K}^{\prime}\right)-\mathbf{\Delta} \mathbf{S}_{\mathbf{I}} / \mathbf{R}$ & $\ln \left(\mathbf{K}^{\prime}\right)-\Delta \mathbf{S}_{\mathbf{R}} / \mathbf{R}$ \\
\hline 4 wt.\% AgNW-90 & 24.5 & 8.93 & -15.5 & -11.1 & -0.72 \\
\hline 5 wt.\% AgNW-90 & 19.1 & 10.4 & -8.76 & -8.65 & -0.68 \\
\hline 6 wt.\% AgNW-90 & 27.1 & 4.56 & -22.6 & -11.9 & 1.31 \\
\hline 7 wt.\% AgNW-90 & 21 & 15.6 & -5.45 & -9.13 & -2.31 \\
\hline
\end{tabular}

Table 4. 12: Activation enthalpy, and activation entropy during recovery process for different silver nanowire (AgNWs-40) contents.

\begin{tabular}{|c|c|c|c|c|c|}
\hline Sample & $\boldsymbol{\Delta H}_{\mathbf{I}}(\mathbf{K J})$ & $\boldsymbol{\Delta H}_{\mathbf{R}}(\mathbf{K J})$ & $\boldsymbol{\Delta} \mathbf{H}(\mathbf{K J})$ & $\ln \left(\mathbf{K}^{\prime}\right)-\mathbf{\Delta} \mathbf{S}_{\mathbf{I}} / \mathbf{R}$ & $\ln \left(\mathbf{K}^{\prime}\right)-\boldsymbol{\Delta} \mathbf{S}_{\mathbf{R}} / \mathbf{R}$ \\
\hline 0.04 wt.\% AgNW-40 & 32.5 & 24.2 & -8.30 & -15.1 & -10.8 \\
\hline 0.25 wt.\% AgNW-40 & 32.2 & 35.8 & 3.60 & -14.9 & -15.1 \\
\hline 0.5 wt.\% AgNW-40 & 32.4 & 26.9 & -5.42 & -14.8 & -10.7 \\
\hline 0.75 wt.\% AgNW-40 & 28.4 & 4.00 & -24.4 & -13.3 & -1.24 \\
\hline 1 wt.\% AgNW-40 & 31.4 & 7.41 & -24.0 & -14.3 & -1.94 \\
\hline 1.25 wt.\% AgNW-40 & 30.8 & 2.20 & -28.6 & -14.2 & -0.09 \\
\hline 1.5 wt.\% AgNW-40 & 26.5 & 4.21 & -22.2 & -12.4 & -0.59 \\
\hline 3 wt.\% AgNW-40 & 19.7 & 6.85 & -12.9 & -9.04 & 0.26 \\
\hline 4 wt.\% AgNW-40 & 24.2 & 17.9 & -6.21 & -10.5 & -3.48 \\
\hline 5 wt.\% AgNW-40 & 21.0 & 11.5 & -9.52 & -9.19 & -0.68 \\
\hline 6 wt.\% AgNW-40 & 15.7 & 9.74 & -6.00 & -6.95 & 0.24 \\
\hline
\end{tabular}

\subsection{Printed Pattern Characterization}

In order to examine the orientation of the AgNWs in the printed patterns, and the quality of printing SEM was used. Figure 4. 45, and Figure 4. 46 show the SEM images of the printed patterns of 6 wt.\% of AgNWs-90 and AgNWs-40, respectively. The images of the 50 wt.\% of synthesized AgNWs, and 50 wt.\% of commercial AgNWs ink are shown in Figure 4. 47.

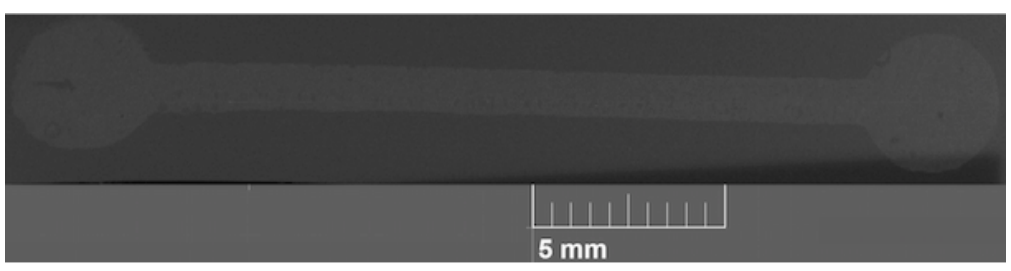

Figure 4. 45: SEM image of the printed pattern of 6 wt.\% AgNWs-90. 


\section{$\lfloor|\perp||||||\perp|$}

$10 \mathrm{~mm}$

Figure 4. 46: SEM image of the printed pattern of $6 \mathrm{wt} . \%$ AgNWs-40.
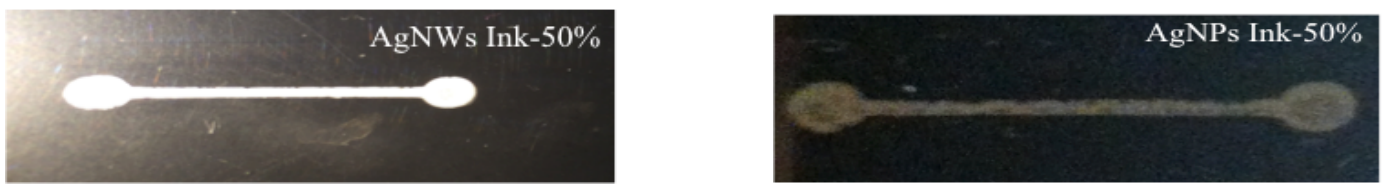

Figure 4. 47: Image of the printed patterns of synthesized AgNWs and commercial AgNPs ink. To see the sharpness of the printed line, Figure 4. 48 and Figure 4. 49 show the SEM images of the line parts of the printed patterns of 6 wt. $\%$ of AgNWs-90 and AgNWs-40, respectively.

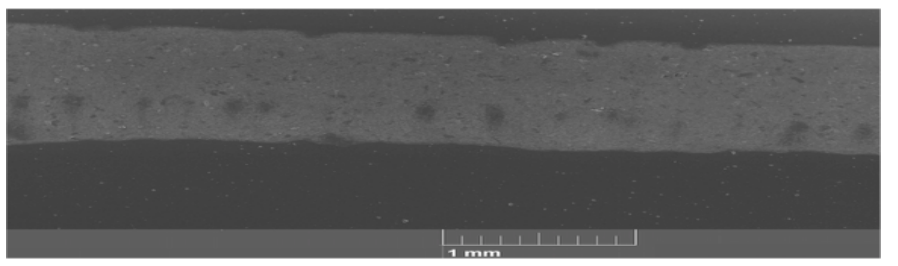

Figure 4. 48: SEM image of the line part in the printed pattern of 6 wt.\% AgNWs-90 (scale bar: $1 \mathrm{~mm})$.

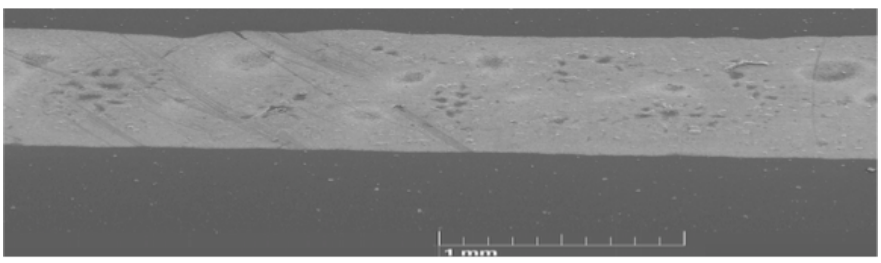

Figure 4. 49: SEM image of the line part in the printed pattern of 6 wt.\% AgNWs-40 (scale bar: $1 \mathrm{~mm})$.

These SEM images illustrate that at low content of AgNWs compare to the commercially available silver inks there is a good quality of printing with sharp line definition.

Figure 4. 50 shows the SEM images of the printed patterns of AgNWs-90 at several contents of AgNWs from 3 to 7 wt.\% at higher magnification. Similar images for AgNWs-40 at different contents of AgNWs from 3 to 6 wt.\% are shown in Figure 4. 51. 

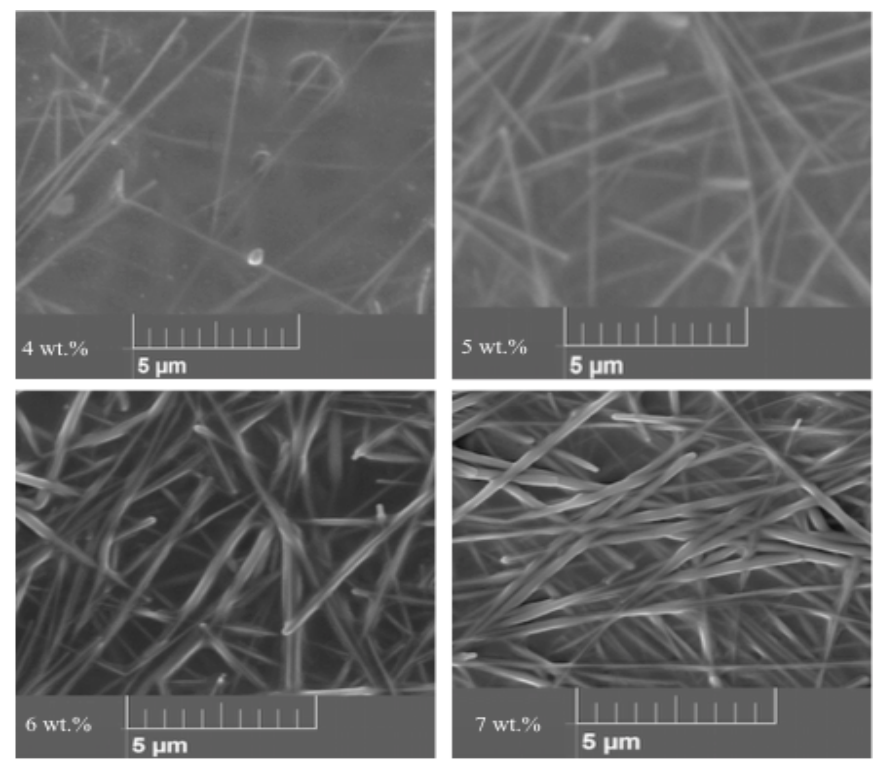

Figure 4. 50: SEM images of the printed patterns of AgNWs-90 with different contents of AgNWs at high magnification (scale bar: $5 \mu \mathrm{m}$ ).
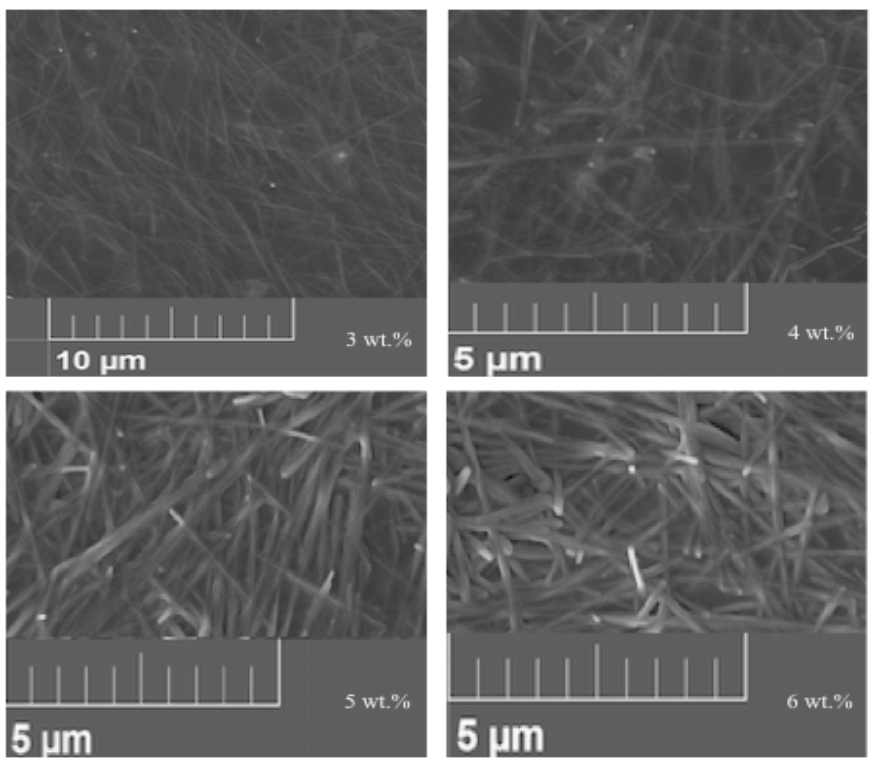

Figure 4. 51: SEM images of the printed patterns of AgNWs-40 with different contents of AgNWs at high magnification (scale bar: $5 \mu \mathrm{m}$ ).

\subsection{Resistivity Measurements}

Table 4. 13 and Table 4. 14 show the resistivity values of the conductive printed patterns at several contents of AgNWs for AgNWs-90 and AgNWs-40, respectively. These results show that how higher contents of AgNWs can decrease the resistivity. Moreover, AgNWs-40 with 
smaller diameter and higher aspect ratio show lower resistivity compared to the AgNWs-90 at same wt.\% content of AgNWs. More importantly, 6 wt.\% of AgNWs inks show the resistivity as low as 70, and $50 \Omega$ for AgNWs-90 and AgNWs-40, respectively. The resistivity values of the conductive printed patterns of the 50 wt.\% synthesized AgNWs and 50 wt.\% commercial AgNPs are also shown in Table 4. 15. These results show how the high aspect ratio (longer and thinner) AgNWs application can decrease the resistivity compare to the silver flake and silver nanoparticle inks.

Table 4. 13: Resistivity measurement for printed patterns at several contents of AgNWs-90.

\begin{tabular}{|c|c|}
\hline AgNWs Content (Wt. \%) & Resistivity ( $\mathbf{\Omega})$ \\
\hline 4 & 175 \\
\hline 5 & 165 \\
\hline 6 & 70 \\
\hline 7 & 36 \\
\hline
\end{tabular}

Table 4. 14: Resistivity measurement for printed patterns at several contents of AgNWs-40.

\begin{tabular}{|c|c|}
\hline AgNWs Content (Wt. \%) & Resistivity ( $\mathbf{( )})$ \\
\hline 3 & 233 \\
\hline 4 & 122 \\
\hline 5 & 65 \\
\hline 6 & 50 \\
\hline
\end{tabular}

Table 4. 15: Resistivity measurement for printed patterns synthesized AgNWs and commercial AgNPs ink.

\begin{tabular}{|c|c|}
\hline Ink & Resistivity \\
\hline 50 wt. \% Synthesized AgNWs & $93 \Omega$ \\
\hline 50 wt. \% Commercial AgNPs & $72 \mathrm{k} \Omega$ \\
\hline
\end{tabular}




\section{CHAPTER 5}

\section{CONCLUSION AND RECOMMENDATIONS FOR FUTURE WORK}

\subsection{Conclusion}

The focus of this work was synthesis and characterization of silver nanowires (AgNWs) and formulation of inks adaptable for economical screen printing process for conductive patterns with sharp line definition. The project was organized in to the following research tasks.

- Synthesis and characterization of AgNWs by a polyol process.

- Scale-up of the synthesis process.

- Determination of the nucleation and growth process during synthesis.

- Parametric study and sensitivity analysis of the synthesis process.

- Optimization of synthesis with respect to nanowire yield.

- Synthesis of AgNWs in a continuous laminar-flow reactor.

- Formulation of AgNWs water-based inks adaptable for economical screen printing.

- Investigation of the rheological behavior of the formulated inks.

- Dimensional analysis and correlation of the time-dependent rheological behavior of the inks under conditions similar to those of screen printing.

- Screen printing and characterization of the printed patterns.

In this study, AgNWs were first synthesized on a small scale by a polyol process as described in previous works. Most of those mentioned the difficulties and challenges in controlling polyol synthesis of AgNWs in batch reactor, especially in large-scale synthesis, and those challenges were confirmed in this work. Subsequently, the process was scaled up by a factor of twenty times 
by determining an optimum combination of reaction volume and string rate to provide uniform a reaction environment. The AgNWs were successfully synthesized by the polyol process in both small and large-scale batch reactors. The AgNWs were characterized by Scanning Electron Microscopy (SEM) to investigate the morphology, diameter, and length of the synthesized AgNWs. These geometric factors were assessed quantitatively through image analysis using image J software.

It is essential to know the reaction mechanism in different steps of the reaction to produce unidirectional crystal growth on the nanometer scale. A three-step reaction mechanism consisting of reduction, nucleation, and growth steps was considered by extraction and characterization of nanostructures during different steps of the synthesis.

Based on previous studies, implementation of an extensive parametric study and sensitivity analysis over the polyol synthesis of AgNWs would be essential to determine the most important factors controlling the process, and to have optimum control over the reaction conditions. Parametric studies on the batch polyol synthesis in this study demonstrated that the most important parameters in the process are reaction temperature and reagent concentrations.

There have not been yet any studies regarding optimization of polyol synthesis of AgNWs to maximize yield. Since a full investigation of the effects of interactions between all of the essential parameters based on Full Factorial Design (FFD) required a very large number of experiments, Design of Experiments (DoE) procedures could be used to not only investigate the simultaneous effect of the different parameters but to determine the optimize reaction condition 
to maximize yield as well. Implementation of DoE established the optimum reaction conditions as $\mathrm{T}=157.6^{\circ} \mathrm{C},\left[\mathrm{AgNO}_{3}\right]=0.102 \mathrm{M},[\mathrm{PVP}]=0.124 \mathrm{M}$, and $\left[\mathrm{CuCl}_{2}\right]=5.16 \mathrm{mM}$ at which the yield of AgNWs is approximately 95\%. Scanning Electron Microscopy (SEM) examination of wires synthesized at the optimum condition confirmed the expected yield.

Based on the previous researches and studies, the polyol synthesis of AgNWs is time consuming and there is a significant batch-to-batch variation. Although the AgNWs are commercially available, they are expensive. Moreover, it is beneficial to synthesize high aspect ratio AgNWs in a rapid way compare to the previous time consuming method such as Successive Multiple Growth (SMG) mechanism. All of these illustrate the necessity to synthesis high aspect ratio of AgNWs in simple, continuous, and inexpensive manner. The application of millifluidic reactors in this study showed that AgNWs with high aspect ratio could be synthesized at lower temperature continuously in a small reactor volume in comparison to batch synthesis.

Application of water as solvent to formulate silver based conductive inks allows the formulation a water-based environmentally friendly conductive ink instead of application of different inorganic and organic solvents. Moreover, utilizing high aspect ratio AgNWs to formulate ink lowers the cost of ink through application of the smaller loading required of high aspect ratio AgNWs compared to low aspect ratio AgNWs, AgNPs, or micron size silver particles. Finally, the application of high aspect ratio of AgNWs can dramatically reduce the sintering temperature, which provides the application of those inks on flexible substrates such as paper and plastic. Water-based AgNWs ink suitable for screen printing; as reliable, fast, inexpensive, and 
environmentally friendly method; was formulated with AgNWs synthesized here and with commercial AgNWs.

The printability of formulated water-based AgNWs inks strongly depends on their rheological behavior. An extensive investigation of the rheological behavior of the ink would be beneficial to control the screen printing process, which is required to create patterns with sharp line definition. Several rheological tests including the Peak Hold (PH), Steady State Flow (SSF), Stress Sweep (SS) and Frequency Sweep (FS) were used to investigate the rheological behavior of the formulated inks. The rheological results showed shear thinning thixotropic behavior produced by AgNWs rather than rheological agents. The PH test was utilized to simulate the screen printing process and mimic different steps of the printing process including charging, through-printing and recovery.

The understanding and investigation of the recovery mechanism after screen printing as the most important interval of the screen printing that is responsible for the printed pattern specifications is inevitable to control the printing process. This investigation should provide quantitative parameters to explain the recovery mechanisms, which hasn't been investigated in previous studies comprehensively. In this study, the results from PH tests were fitted by the Stretched Exponential model (SEmo) to introduce the characteristic time for the recovery process after screen printing. The activation energy consideration and Peclet number calculation for the recovery process proved that the recovery mechanism would occur through thermodynamic and hydrodynamic interactions such as wire-wire forces, wire-fluid forces, and viscous forces rather than Brownian motion. The activation enthalpy and entropy consideration for the recovery 
process showed that the shear thinning behavior of the inks arising from the oriented arrangements of wires, which corresponds to a negative value of activation entropy would be compensated by a decrease in activation enthalpy. The transition from high shear to low shear during the recovery process is controlled by wire-wire interactions, which results in a negative activation enthalpy $(\Delta \mathrm{H})$. The results show that viscosity recovery after screen printing is controlled by the very high aspect ratio of AgNWs.

Even patterns and sharp line definition are the most important desired characteristics of the printed circuits. In this study, Scanning Electron Microscopy (SEM) was used to characterize the printed patterns and investigate the quality of printing. The formulated inks with several AgNWs contents were printed on the polycarbonate substrates with a manual screen printer. SEM images of the patterns show that printing with moderate quality is possible at AgNWs contents as low as 3 wt. $\%$.

It is essential to assess how the low content of the high aspect ratio of AgNWs can boost the conductivity of the printed patterns. The resistivity measurements of the printed patterns of the formulated inks with silver nanowire content as low as $3 \mathrm{wt} . \%$ showed how the application of high aspect ratio of AgNWs could provide high conductivity at low content of AgNWs, which is difficult to achieve with AgNPs, micron meter size silver particles, or silver flake at the same content. These results provide a route to formulate inexpensive silver based ink adaptable for economical screen printing process. 


\subsection{Recommendations for Future Work}

The following recommendations are suggested to further study.

1. Optimization of the morphology of the synthesized AgNWs in a millifluidic reactor through the reaction condition modifications in flow rate and residence time.

2. Large-scale and programmable synthesis of AgNWs in millifluidic reactor by alternating the reagent injection and tube washing through utilizing programmable large syringe pumps.

3. Kinetic investigation of the Polyol process through quantification of the nucleation and growth mechanisms of millifluidic polyol process, and size evolution of silver nanostructures in millifluidic reactor to prove how the morphology and particle size can be controlled better through millifluidic rector compare to the batch one.

4. Modeling of the rheological behavior of the AgNWs ink to predict and control suspension diffusion, rheological properties and particle microstructure, develop the code based on the simple interaction, and calibrate the results of the simulation with the present experimental results in this study.

5. Coating and printing AgNW inks with different dimensions to measure dimension dependent transmittance and sheet resistance, and using a percolation model to explain the relation between $\mathrm{AgNWs}$ dimensions and sheet resistance.

6. Sintering of the printed patterns through heating, drying at room temperature. Decreasing resistivity without sintering by solution treatment. Measuring film thickness, shrinkage, specific sheet resistance, and electrical resistance as functions of sintering and treatment conditions to evaluate the optimum sintering process.

7. Practical application of AgNWs conductive patterns for solar panel innovation. 
8. Investigation of the application of screen printed of AgNWs arrays on paper substrate specially for Surface Enhance Raman Scattering (SERS) application to create a highly sensitive, cheap, flexible, and efficient SERS substrate for biomolecule, pollutant, and pesticide detection. 


\section{NOMENCLATURE}

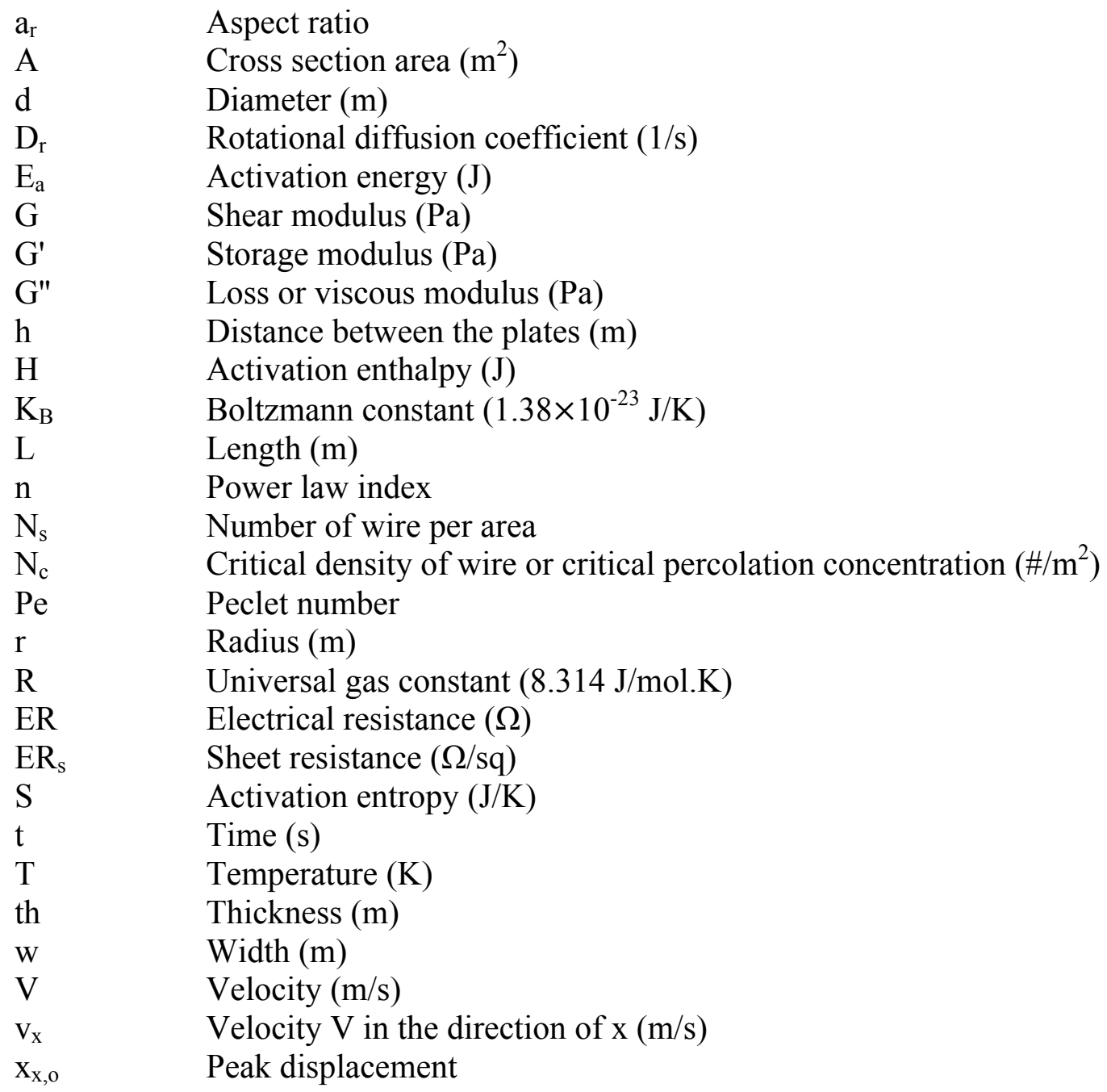




\section{Greek Letters}

$\beta \quad$ Dimensionless constant

$\dot{\gamma} \quad$ Shear rate $(1 / \mathrm{s})$

$\delta \quad$ Loss angle

$\Delta \quad$ Change of quantity

$\varepsilon \quad$ Dimensionless constant

$\eta \quad$ Viscosity (Pa.s)

$\eta_{0} \quad$ Steady viscosity at high shear rate (Pa.s)

$\eta_{\infty} \quad$ Steady viscosity at low shear rate (Pa.s)

$\eta_{\mathrm{I}} \quad$ Initial viscosity (Pa.s)

$\eta_{\mathrm{R}} \quad$ Recovered viscosity (Pa.s)

$\rho \quad$ Electrical resistivity $(\Omega \mathrm{m})$

$\tau \quad$ Characteristic time (s)

$\sigma \quad$ Shear stress $(\mathrm{Pa})$

$v \quad$ Number density of wires $\left(\# / \mathrm{m}^{3}\right)$

$\omega \quad$ Angular frequency $(\mathrm{rad} / \mathrm{s})$

$\Omega \quad$ Rotational speed ( $\mathrm{rad} / \mathrm{s})$

\section{ABREVIATION}

1D One-Dimensional

AFM Atomic Force Microscopy

BBD Box-Behnken Design

CMC Carboxymethyl Cellulose

CNTs Carbon Nanotubes

DI Deionized

DoE Design of Experiment

DSC Differential Scanning Calorimetry

EDAX X-Ray Energy Dispersive Analysis

EG Ethylene Glycol

fcc face-center-cubic

FESEM Field Emission Scanning Electron Microscopy

FFD Full Factorial Design

FS Frequency Sweep

FTIR Fourier Transform Infrared Spectroscopy

GA Glycol Aldehyde

HRTEM High Resolution Transmission Electron Microscopy

IPA Isopropyl Alcohol

ITO Indium-Tin Oxide

LEDs Light Emitting Diodes

LCDs Liquid Crystal Displays 
LTCC Low Temperature Co-fired Ceramic

LVO Low-Volatile Organic

LVR Linear Visco-elastic Region

MFM Magnetic Field Microscopy

MTPs Multiply Twinned Particles

OLEDs Organic Light Emitting Diodes

PDMS Poly Dimethyl Siloxane

PEs $\quad$ Printed Electronics

PH Peak Hold

PTEF Poly Tetra Fluoro Ethylene

PTFs Polymer Thick Films

PVP Poly(Vinyl Pyrrolidone)

RFID Radio Frequency Identification

RSD Response Surface Design

SEmo Stretched Exponential Model

SEM Scanning Electron Microscopy

SERS Surface Enhanced Raman Scattering

SMG Successive Multiple Growth

SS Stress Sweep

SSF Steady State Flow

SThM Scanning Thermal Microscopy

STM Scanning Tunneling Microscopy

STP Scanning Tunneling Probes

TC Transparent Conductor

TEM Transmission Electron Microscopy

TGA Thermo Gravimetric Analysis

TG-DSC Thermo Gravimetric-Differential Scanning Calorimetry

TNT Titanate Nanotube

XRD X-Ray Diffraction 


\section{REFRENCES}

[1] Kylee E. Korte, Sara E. Skrabalak, and Younan Xia, "Rapid synthesis of silver nanowires through a CuCl- or $\mathrm{CuCl}_{2}$-mediated polyol process", J. Mater. Chem. 2008, 18, 437-441.

[2] Jin Hwan Lee, Phillip Lee, Dongjin Lee, Seung Seob Lee, and Seung Hwan Ko, "Large-Scale Synthesis and Characterization of Very Long Silver Nanowires via Successive Multistep Growth", Cryst. Growth Des. 2012, 12, 5598-5605.

[3] Weiwei Yin, Dong-Hyun Lee, Jaesoo Choi, Chinho Park, and Sung Min Cho, "Screen printing of silver nanoparticle suspension for metal interconnects", Korean J. Chem. Eng. 2008, 25(6), 1358-1361.

[4] Rita Faddoul, Nadège Reverdy-Bruas, Anne Blayo, "Formulation and screen printing of water based conductive flake silver pastes onto green ceramic tapes for electronic applications", Mater. Sci. Eng. 2012, B 177, 1053-1066.

[5] Rita Faddoul, Nade'ge Reverdy-Bruas, Jose' phine Bourel, "Silver content effect on rheological and electrical properties of silver pastes", J Mater Sci: Mater Electron 2012, 23:1415-1426.

[6] Yi Li . Daniel Lu and C.P. Wong, "Electrical Conductive Adhesives with Nanotechnologies", Springer New York Dordrecht Heidelberg London 2010.

[7] Nicoleta Lupu, "Nanowires Science and Technology", Intech 2010.

[8] M. S. Dresselhausa, Y.M. Linb, O. Rabinc, M.R. Blackb, G. Dresselhaus, "Nanowires", Massachusetts Institute of Technology 2003.

[9] Chang Chen, Li Wang, Guohua Jiang, Junfeng Zhou, Xu Chen, Haojie Yu and Qiang Yang, "Study on the synthesis of silver nanowires with adjustable diameters through the polyol process", Nanotechnology 2006, 17, 3933-3938.

[10] Yugang Sun, Byron Gates, Brian Mayers, and Younan Xia, "Crystalline Silver Nanowires by Soft Solution Processing”, Nano Lett. 2002, Vol. 2, No. 2 165-168.

[11] Benjamin Wiley, Yugang Sun, Younan Xia, "Synthesis of Silver Nanostructures with Controlled Shapes and Properties”, Acc. Chem. Res. 2007, 40, 1067-1076.

[12] V. Rodrigues, J. Bettini, A. R. Rocha, L. G. C. Rego, and D. Ugarte, "Quantum conductance in silver nanowires: Correlation between atomic structure and transport properties", Phys. Rev. B 2002, 65, 153402.

[13] Ho Seok Lee, Yeon Won Kim, Jong Eun Kim, Sung Woon Yoon, Tae Young Kim, Jin-Seo Nohe, and Kwang S. Suh, "Synthesis of dimension-controlled silver nanowires for highly conductive and transparent nanowire films", Acta Materialia 2015, 83, 84-90.

[14] Sukanta De, Thomas M. Higgins, Philip E. Lyons, Evelyn M. Doherty, Peter N. Nirmalraj, Werner J. Blau, John J. Boland, and Jonathan N. Coleman, "Silver Nanowire Networks as Flexible, Transparent, Conducting Films: Extremely High DC to Optical Conductivity Ratios", ACS Nano 2009, Vol. 3, N. 7, 1767-1774.

[15] Anuj R. Madaria, Akshay Kumar, Fumiaki N. Ishikawa, and Chongwu Zhou, "Uniform, Highly Conductive, and Patterned Transparent Films of a Percolating Silver Nanowire Network on Rigid and Flexible Substrates Using a Dry Transfer Technique”, Nano Res. 2010, 3: 564-573. [16] Haibo Mao, Jinyang Feng, Xiao Ma, Can Wu, Xiujian Zhao, "One-dimensional silver nanowires synthesized by self-seeding polyol process”, J Nanopart Res. 2012, 14:887. 
[17] Cheng Yang, Youhong Tang, Zijin Su, Zhexu Zhang, Cheng Fang, "Preparation of Silver Nanowires via a Rapid, Scalable and Green Pathway", J. Mater. Sci. Technol. 2015, 31(1), 1622.

[18] Liangbing $\mathrm{Hu}$, Han Sun Kim, Jung-Yong Lee, Peter Peumans, and Yi Cui, "Scalable Coating and Properties of Transparent, Flexible, Silver Nanowire Electrodes", ACS Nano 2010, Vol. 4, NOo. 5, 2955-2963.

[19] Xiaojian Yang, Wei He, Shouxu Wang, Guoyun Zhou, Yao Tang, Juanhong Yang, "Effect of the different shapes of silver particles in conductive ink on electrical performance and microstructure of the conductive tracks", J Mater Sci: Mater Electron 2012, 23:1980-1986.

[20] A.B.V. Kiran Kumar, Chang wan Bae, Longhai Piao, Sang-Ho Kim, "Silver nanowire based flexible electrodes with improved properties: High conductivity, transparency, adhesion and low haze", Mater. Res. Bull. 2013, 48, 2944-2949.

[21] G. Khanarian, J. Joo, X. Q. Liu, P. Eastman, D. Werner, K. O'Connell, and P. Trefonas, "The optical and electrical properties of silver nanowire mesh films", J. Appl. Phys. 2013, 114, 024302.

[22] Liangbing $\mathrm{Hu}$, Hui $\mathrm{Wu}$, and Yi Cui, "Metal nanogrids, nanowires, and nanofibers for transparent electrodes", MRS Bulletin, 2011, 36.

[23] Jinting Jiu, Takehiro Tokuno, Masaya Nogi, Katsuaki Suganuma, "Synthesis and application of Ag nanowires via a trace salt assisted hydrothermal process", J Nanopart Res. 2012, 14:975.

[24] Simona E. Hunyadi Murph, Catherine J. Murphy, Austin Leach, and Kenneth Gall, "A Possible Oriented Attachment Growth Mechanism for Silver Nanowire Formation", Cryst. Growth Des. 2015, 15, 1968-1974.

[25] Shohreh Hemmati, Dale P. Barkey, Nivedita Gupta, and Ryan Banfield, "Synthesis and Characterization of Silver Nanowire Suspensions for Printable Conductive Media", ECS J. Solid State Sci. Technol. 2015, 4 (4) P3075-P3079.

[26] Sara E. Skrabalak, Benjamin J. Wiley, Munho Kim, Eric V. Formo, and Younan Xia, "On the Polyol Synthesis of Silver Nanostructures: Glycolaldehyde as a Reducing Agent", Nano Lett. 2008, Vol. 8, No. 7, 2077-2081.

[27] Mohd Rafie Johan, Nurul Azri Khalisah Aznan, Soo Teng Yee, Ing Hong Ho, Soo Wern Ooi, Noorsaiyyidah Darman Singho, and Fatihah Aplop, "Synthesis and Growth Mechanism of Silver Nanowires through Different Mediated Agents $\left(\mathrm{CuCl}_{2}\right.$ and $\left.\mathrm{NaCl}\right)$ Polyol Process", Hindawi Publishing Corporation Journal of Nanomaterials 2014.

[28] Jie-Jun Zhu, Cai-Xia Kan, Jian-Guo Wan, Min Han, and Guang-Hou Wang, "High-Yield Synthesis of Uniform Ag Nanowires with High Aspect Ratios by Introducing the Long-Chain PVP in an Improved Polyol Process", Hindawi Publishing Corporation J. Nanomater 2011.

[29] Lakshminarayana Polavarapu, Kiran Kumar Manga, Hanh Duyen Cao, Kian Ping Loh, and Qing-Hua Xu, "Preparation of Conductive Silver Films at Mild Temperatures for Printable Organic Electronics", Chem. Mater. 2011, 23, 3273-3276.

[30] Yana Aleeva and Bruno Pignataro, "Recent advances in upscalable wet methods and ink formulations for printed electronics", J. Mater. Chem. C, 2014, 2, 6436.

[31] Samali Datta, Kristen Keller, Douglas L. Schulz, and Dean C. Webster, "Conductive Adhesives From Low-VOC Silver Inks for Advanced Microelectronics Applications", IEEE Transactions on Components, Packaging, and Manufacturing Technology 2011, Vol. 1, No. 1. 
[32] Benjamin J. Wiley, Zenghui Wang, Jiang Wei, Yadong Yin, David H. Cobden, and Younan Xia , "Synthesis and Electrical Characterization of Silver Nanobeams", Nano Letter 2006, Vol. 6, No. 10, 2273-2278.

[33] Jan Mewis, Norman J. Wagner, “Colloidal Suspension Rheology”, Cambridge University Press 2015.

[34] A. Franck, "Understanding Rheology of Structured Fluids", TA Instruments.

[35] Sabuj Mallik, "Study of the Time-dependent Rheological Behaviour of Lead-free Solder Pastes and Flux Mediums used for Flip-Chip Assembly Applications", Electronics Manufacturing Engineering Research Group School of Engineering, University of Greenwich at Medway Kent, UK 2009.

[36] Howard A. Barnes, "Thixotropy a review”, J. Non-Newtonian Fluid Mech. 1997, 70,1-33.

[37] Xihong Peng, "Nanowires - Recent Advances", Intech 2012.

[38] David W. Litchfield and Donald G. Baird, "The Rheology of High Aspect Ratio NanoParticle Filled Liquids", Rheol. Reviews 2006, pp $1-60$.

[39] D. Quemada, "Rheological modeling of complex fluids. I. The concept of effective volume fraction revisited", Eur. Phys. J. 1998, AP 1, 119-127.

[40] Philippe Cassagnau, "Linear viscoelasticity and dynamics of suspensions and molten polymers filled with nanoparticles of different aspect ratios", Polymer 2013, 54, 4762-4775.

[41] H.D. Chandler, "An activation energy approach to analysing non-Newtonian slurry viscosities with application to a suspension of starch in a carboxymethylcellulose solution", Powder Technol. 2014, 268, 368-372.

[42] Cai-Hong Liu, Xun Yu, "Silver nanowire-based transparent, flexible, and conductive thin film”, Nanoscale Res. Lett. 2011, 6:75.

[43] Waynie M. Schuette and William E. Buhro, "Polyol Synthesis of Silver Nanowires by Heterogeneous Nucleation; Mechanistic Aspects Influencing Nanowire Diameter and Length”, Chem. Mater. 2014, 26, 6410-6417.

[44] Guh-Hwan Lim, Seong Jun Lee, Insung Han, Shingyu Bok, Jung Heon Lee, Jaewook Nam, Jeong Ho Cho, Byungkwon Lim, "Polyol synthesis of silver nanostructures: Inducing the growth of nanowires by a heat-up process", Chem. Phys. Lett. 2014, 602, 10-15.

[45] Ronen Gottesman, Alex Tangy, Ilan Oussadon and David Zitoun, "Silver nanowires and nanoparticles from a millifluidic reactor: application to metal assisted silicon etching", New J. Chem. 2012, 36, 2456-2459.

[46] Nguyen V. Nghia, Nguyen N. K. Truong1, Nguyen M. Thong, Nguyen P. Hung, "Synthesis of Nanowire-Shaped Silver by Polyol Process of Sodium Chloride”, Int. J. Mater. Chem. 2012, 2(2): 75-78.

[47] Yuan-Jun Song1, Mingliang Wang, Xiao-Yang Zhang, Jing-Yuan Wu, and Tong Zhang, "Investigation on the role of the molecular weight of polyvinyl pyrrolidone in the shape control of high-yield silver nanospheres and nanowires", Nanoscale Res. Lett. 2014, 9:17.

[48] Jian-Yang Lin, Yu-Lee Hsueh, Jung-Jie Huang, Jia-Rung Wu, "Effect of silver nitrate concentration of silver nanowires synthesized using a polyol method and their application as transparent conductive films", Thin Solid Films 2015, 584, 243-247.

[49] Sahin Coskun, Burcu Aksoy, and Husnu Emrah Unalan, "Polyol Synthesis of Silver Nanowires: An Extensive Parametric Study", Cryst. Growth Des. 2011, 11, 4963-4969.

[50] Jaime E. Pérez, Adriana B. Arauz, Luis A. García, and José L. Rodríguez, "Synthesis of Silver Nanostructures by the Polyol Method and their Statistical Analysis Using Design of Experiments", MRS 2012, Vol. 1371. 
[51] Keunju Park, Dongseok Seo, Jongkook Lee, "Conductivity of silver paste prepared from nanoparticles”, Colloids and Surfaces A: Physicochem. Eng. Aspects 313-314, 2008, 351-354. [52] Shunhua Wang, Xu Zhang, and Weiwei Zhao, "Flexible, Transparent, and Conductive Film Based on Random Networks of Ag Nanowires", Hindawi Publishing Corporation J. Nanomater 2013.

[53] Takehiro Tokuno, Masaya Nogi, Makoto Karakawa, Jinting Jiu, Thi Thi Nge, Yoshio Aso, and Katsuaki Suganuma, "Fabrication of Silver Nanowire Transparent Electrodes at Room Temperature", Nano Res. 2011, 4(12): 1215-1222.

[54] Jinhwan Lee, Phillip Lee, Hyungman Lee, Dongjin Lee, Seung Seob Leea and Seung Hwan Ko, "Very long Ag nanowire synthesis and its application in a highly transparent, conductive and flexible metal electrode touch panel", Nanoscale 2012, 4, 6408.

[55] Yao Tang, Wei He, Gyoyun Zhou, Shouxu Wang, Xiaojian Yang, Zhihua Tao, and Juncheng Zhou, "A new approach causing the patters fabricated by silver nanoparticles to be conductive without sintering", Nnaotecnology 2012. 23, 355304.

[56] Daisuke Wakuda, Keun-Soo Kim and Katsuaki Suganuma, "Room temperature sintering of Ag nanoparticles by drying solvent", Scripta Materialia 2008, 59, 649-652.

[57] Kwang-Seok Kim, Woo-Ram Myung, and Seung-Boo Jung, "Effects of Sintering Conditions on Microstructure and Characteristics of Screen-Printed Ag Thin Film", Electron. Mater. Lett. 2012, Vol. 8, No. 3, pp. 309-314.

[58] Dongpo Chen, Lei Zhao, Hongwei Diao, Wenbin Zhang, Ge Wang, Wenjing Wang, "Rheological properties and related screen-printing performance of low-temperature silver pastes for a-Si:H/c-Si heterojunction solar cells", J Mater Sci: Mater Electron 2014.

[59] Haisheng Chen, Yulong Ding, Alexei Lapkin, "Rheological behaviour of nanofluids containing tube / rod-like nanoparticles", Powder Technol. 2009, 194, 132-141.

[60] Hu Zhou, Patrick Heyer, Ho-Jong Kim, Jung-Hoon Song, Longhai Piao, and Sang-Ho Kim, "Reversible Macroscopic Alignment of Ag Nanowires", Chem. Mater. 2011, 23, 3622-3627.

[61] Oana Coocirlan, and Olga Iulian, "Density, viscosity and refractive index of the dimethyl sulfoxide + o-xylene system", J. Serb. Chem. Soc. 2099, 74 (3) 317-329.

[62] Jun Liu, Yangyang Gao, Dapeng Cao, Liqun Zhang, and Zhanhu Guo, "Nanoparticle Dispersion and Aggregation in Polymer Nanocomposites: Insights from Molecular Dynamics Simulation", Langmuir 2011, 27, 7926-7933. 